\title{
Population Dose Commitments Due to Radioactive Releases from Nuclear Power Plant Sites in 1988
}

Manuscript Completed: January 1992

Date Published: January 1992

Prepared by

D. A. Baker

Pacific Northwest Laboratory

Richland, WA 99352

Prepared for

Division of Computer and Telecommunications Services

Office of Information Resources Management

U.S. Nuclear Regulatory Commission

Washington, DC 20555

NRC FIN B2243 
1. Population Dose Commitments Due to Radioactive Releases from Nuclear Power Plant Sites in 1975, PNL-2439, October 1977.

2. Population Dose Commitments Due to Radioactive Releases from Nuclear Power Plant Sites in 1976, NUREG/CR-1125, PNL-2940, December 1979.

3. Population Dose Commitments Due to Radioactive Releases from Nuclear Power Plant Sites in 1977, NUREG/CR-1498, PNL-3324, October 1980.

4. Population Dose Commitments Due to Radioactive Releases from Nuclear Power Plant Sites in 1978, NUREG/CR-2201, PNL-4039, June 1982.

5. Population Dose Commitments Due to Radioactive Releases from Nuclear Power Plant Sites in 1979, NUREG/CR-2850, PNL-4221, Vol. 1, December 1982.

6. Population Dose Commitments Due to Radioactive Releases from Nuclear Power PTant Sites in 1980, NUREG/CR-2850, PNL-4221, Vol 2, August 1983.

7. Population Dose Commitments Due to Radioactive Releases from Nuclear Power Plant Sites in 1981, NUREG/CR-2850, PNL-4221, Vol. 3, January 1985.

8. Population Dose Commitments Due to Radioactive Releases from Nuclear Power Plant Sites in 1982, NUREG/CR-2850, PNL-4221, Vol. 4, June 1986.

9. Population Dose Commitments Due to Radioactive Releases from Nuclear Power Plant Sites in 1983, NUREG/CR-2850, PNL-4221, Vol. 5, January 1987.

10. Population Dose Comritments Due to Radioactive Releases from Nuclear Power Plant Sites in 1984, NUREG/CR-2850, PNL-4221, Vo1. 6, January 1988.

11. Population Dose Commitments Due to Radioactive Releases from Nuclear Power Plant Sites in 1985, NUREG/CR-2850, PNL-4221, Vo1. 7, August 1988.

12. Population Dose Commitments Due to Radioactive Releases from Nuclear Power Plant Sites in 1986, NUREG/CR-2850, PNL-4221, Vo1. 8, September 1989

13. Population Dose Cominitments Due to Radioactive Releases from Nuclear Power Plant Sites in 1987, NUREG/CR-2850, PNL-4221, Vol. 9, August 1990 


\section{ABSTRACT}

Population radiation dose commitments have been estimated from reported radionuclide releases from commercial power reactors operating during 1988. Fifty-year dose commitments for a one-year exposure from both liquid and atmospheric releases were calculated for four population groups (infant, child, teen-ager and adult) residing between 2 and $80 \mathrm{~km}$ from each of 71 reactor sites. This report tabulates the results of these calculations, showing the dose commitments for both water and airborne pathways for each age group and organ. Also included for each of the sites is a histogram showing the fraction of the total population within 2 to 80 $\mathrm{km}$ around each site receiving various average dose commitments from the airborne pathways.

The total collective dose commitments (from both liquid and airborne pathways) for each site ranged from a high of 16 person-rem to a low of 0.0011 person-rem for the sites with plants operating throughout the year with an arithmetic mean of 1.1 person-rem. The total population dose for all sites was estimated at 75 person-rem for the 150 million people considered at risk.

The site average individual dose commitment from all pathways ranged from a low of $3 \times 10^{-7}$ mrem to a high of 0.02 mrem. No attempt was made in this study to determine the maximum dose commitment received by any one individual from the radionuclides released at any of the sites. However, licensee calculation of doses to the maximally exposed individual at some sites indicated values of up to approximately 100 times average individual doses (on the order of a few millirem per year). 


\section{CONTENTS}

ABSTRACT . . . . . . . . . . . . . . . . . . .

ACKNOWLEDGMENTS ........................ . . $x i$

INTRODUCYION . . . . . . . . . . . . . . . . . . . 1.1

Site-Dependent Parameters . . . . . . . . . . . . 1.6

RESULTS . . . . . . . . . . . . . . . . . . 1.7

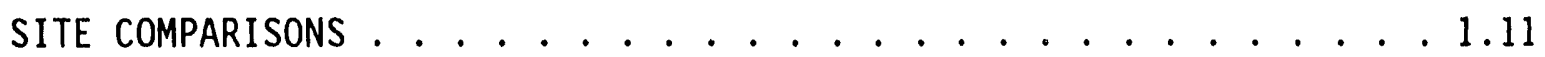

SITE SUMMARIES ...................... . . . . . . . .

Arkansas 0ne $1 \& 2$. . . . . . . . . . . . . . . . 2.2

Beaver Valley $1 \& 2$. . . . . . . . . . . . . . 2.4

Big Rock Point . . . . . . . . . . . . . . . 2.6

Braidwood 1 \& 2 . . . . . . . . . . . . . . . 2.8

Browns Ferry 1, 2, \& 3 . . . . . . . . . . . . 2.10

Brunswick 1\& 2.................... 2. . . . . . 12

Byron 1\&2..................... 2. . . . . . . . .

Callaway ..................... 2..16

Calvert Cliffs $1 \& 2$. . . . . . . . . . . . . 2.18

Catawba $1 \& 2$................... 2. . . . . 20

Clinton........................222

Cook 1\&2 ..................... 2.24

Cooper ........................226

Crystal River 3.................. 2. . . . . . 8

Davis-Besse..................... . . . . . . . . . . . .

Diablo Canyon 1\&2................. . . 2.32 
Dresden $1,2, \& 3 \ldots \ldots \ldots . \ldots \ldots$

Duane Arnold . . . . . . . . . . . . . 2.36

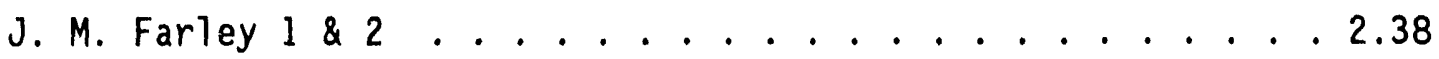

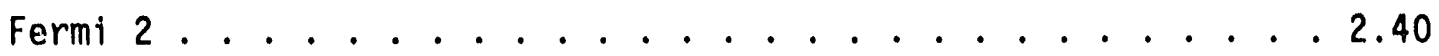

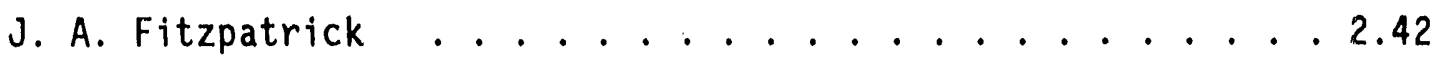

Fort Calhoun . . . . . . . . . . . . . 2.44

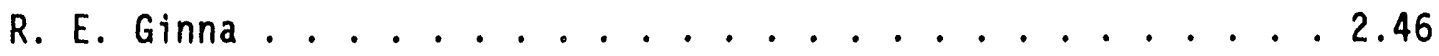

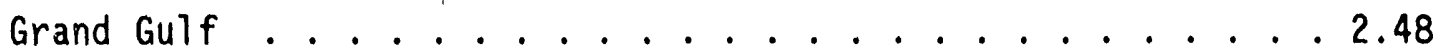

Haddam Neck . . . . . . . . . . . . . . . 2.50

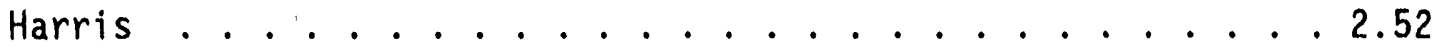

Edwin I. Hatch . . . . . . . . . . . . 2.54

Hope Creek ................... . . 2.56

Indian Point $1,2, \& 3 \ldots \ldots . \ldots . \ldots . \ldots$

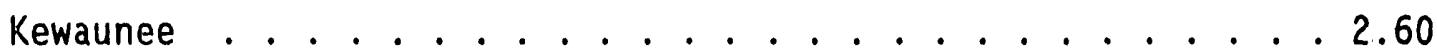

Lacrosse . . . . . . . . . . . . . . 2.62

LaSalle 1 \& 2 . . . . . . . . . . . . 2.64

Limerick . . . . . . . . . . . . . 2.66

Maine Yankee ....................... 2.68

McGuire 1\& $2 \ldots \ldots . \ldots . \ldots . \ldots 2 . \ldots$

Millstone 1, $2 \& 3 \ldots \ldots . \ldots . \ldots . \ldots . \ldots 2 . \ldots$

Monticello .............................. 2.74

Nine Mile Point $1 \& 2 \ldots \ldots . \ldots . . \ldots 2 . \ldots$

North Anna $1 \& 2$. . . . . . . . . . . . 2.78

Oconee $1,2, \& 3 \ldots \ldots \ldots . \ldots \ldots$ 


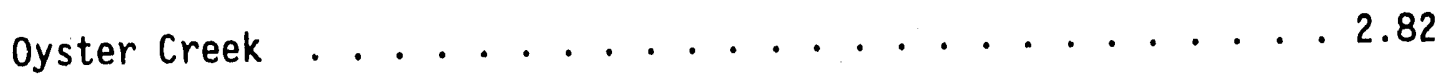

Palisades .................... . . . 2.84

Palo Verde 1, 2, \& $3 \ldots \ldots . \ldots . \ldots . . \ldots 2 . . \ldots$

Peach Bottom $2 \& 3 \ldots \ldots . \ldots . \ldots . \ldots . . \ldots 28$

Perry . . . . . . . . . . . . . . 2.90

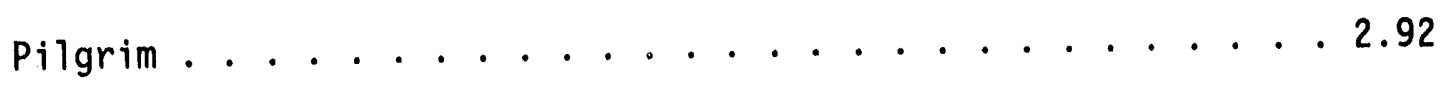

Point Beach $1 \& 2 \ldots \ldots . \ldots . . \ldots . . \ldots 2 . . \ldots$

Prairie Island $1 \& 2 \ldots \ldots . \ldots . \ldots . . \ldots 2 . \ldots 6$

Quad Cities $1 \& 2 \ldots \ldots \ldots . \ldots \ldots$

Rancho Seco . . . . . . . . . . . . . . 2. . . . . . . . .

River Bend . . . . . . . . . . . . . 2. . . . . . . . . .

H. B. Robinson 2......................... . . . .

Saint Lucie $1 \& 2 \ldots \ldots . \ldots . . \ldots . . \ldots 2 . \ldots 6$

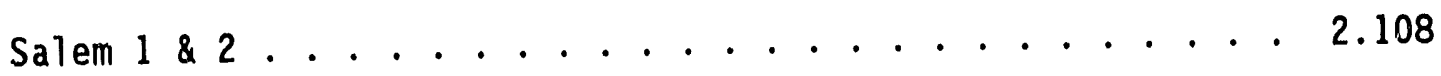

San Onofre 1, 2, \& $3 \ldots \ldots \ldots . \ldots . \ldots . \ldots 2 . \ldots$

Sequoyah $1 \& 2 \ldots \ldots \ldots . \ldots \ldots$

Shoreham ................. 2.114

South Texas ................. . . 2.116

Summer ............................ 2.118

Surry $1 \& 2 \ldots \ldots . \ldots . \ldots . \ldots 2 . \ldots$

Susquehanna $1 \& 2 \ldots \ldots . \ldots . . \ldots . . \ldots 2 . . \ldots 22$

Three Mile Island $1 \& 2 \ldots \ldots . \ldots . \ldots . . \ldots 2 . \ldots$

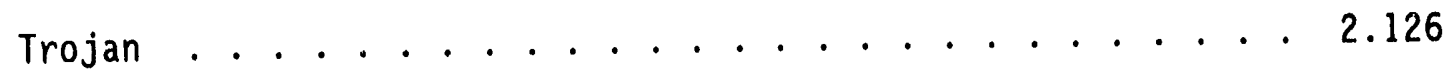

Turkey Point $3 \& 4 \ldots \ldots . \ldots . \ldots . \ldots . . \ldots 28$ 
Vermont Yankee ............... 2.130

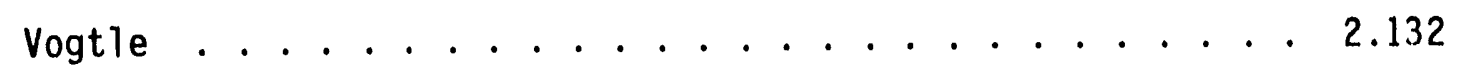

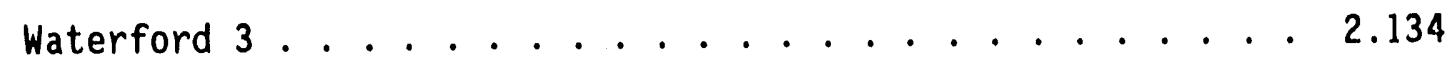

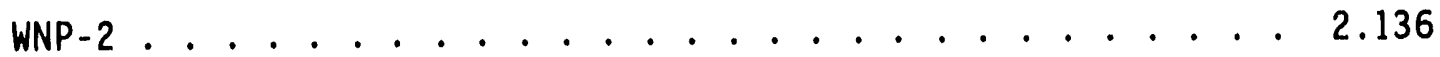

Wolf creek ................. . . 2.138

Yankee Rowe ................. . . 2.140

Zion $1 \& 2 \ldots \ldots . \ldots . \ldots . \ldots 2 . \ldots 2$

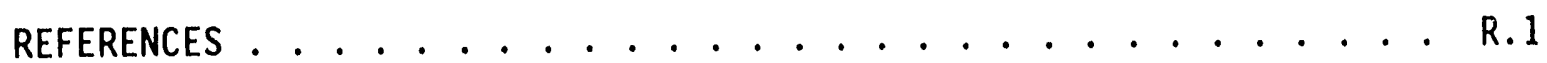

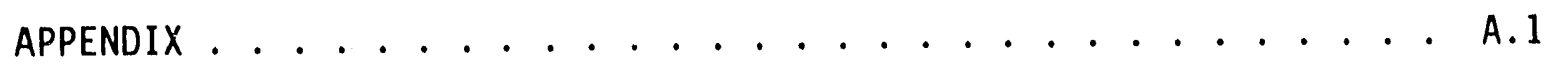




\section{FIGURES}

1. Fraction of Total Population Receiving Various Average Individual Total-Body Dose Commitments for All Sites . . . . 1.8

2. Airborne Population Dose Commitment for the Reactor Sites, 19881.9 


\section{TABLES}

1. Organs Considered in This Study ............... 1.1

2. Pathways Considered in This Study ........... 1.2

3. Environmental Statements for Power Plants Included in This Study 1.3

4. Reactor Characteristics and Population Total-Body

Dose Commitments, 1988 . . . . . . . . . . . . 1.4

5. Comparison of Annual Population Dose Commitments and

Energy Output for the Past 14 Years . . . . . . . . . . 1.11

6. Total-Body Population Doses from Nuclear Power Plant Effluents

During Normal Operations, 1975-1988 . . . . . . . 1.13

7. Average Population Dose for Last Three Years, person-rem . . . 1.14

8. Major Radionuclide Contributions to Population Doses from

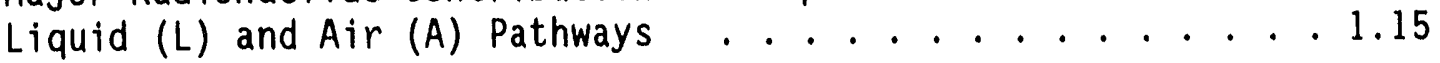




\section{ACKNOWLEDGMENTS}

The author greatly appreciates the development of the population distributions around each site from census data by Donald P. Cleary of the U.S. Nuclear Regulatory Commission. The author also gratefully acknowledges the contributions and suggestions by Richard A. Hartfield, Thomas $H$. Essig, Charles A. Willis, and Kazimieras Campe, also of the U.S. Nuclear Regulatory Commission. 


\section{INTRODUCTION}

A11 commercial nuclear power reactors release small amounts of radioactive materials to the environment during normal operation. Because of these releases, concern was expressed about the magnitude of the collective dose received by the general population esiding around these nuclear power plants. In response to this concern, the Pacific Northwest Laboratory (PNL) (a) contracted with the Nuclear Regulatory Commission (NRC) to undertake a series of studies to estimate radiation dose commitments produced by radionuclide releases from commercial light-water power reactors starting in 1975 (see previous reports in this series, $p$. ii). This document is a continuation of these studies and considers the doses from releases during 1988. In this study, as in those previous, we estimated the collective (population) dose commitment (b) from both the liquid and gaseous releases to four age groups making up the population residing in the region of the site: infant ( 0 to $1 \mathrm{yr}$ ), child (1 to 11 $\mathrm{yr}$ ), teen-ager (11 to $17 \mathrm{yr}$ ), and adult (17 $\mathrm{yr}$ and older).

The particular organs of reference in this study are listed in Table 1. The major pathways by which radionuclides travel from the reactor to the individual receptors are shown in Table 2. Other possible liquid pathways such as direct exposure from waterborne activities (swimming, boating, shoreline recreation) and internal exposure through ingestion of food produced using contaminated irrigation water were not included; we have found from past experience that the doses from these pathways are generally much smaller than the doses from the pathways considered in this study.

TABLE 1. Organs Considered in This Study

Organs Affected by Airborne Releases

Total body

Thyroid

Bone

GI tract

Liver

Lung
Organs Affected by Waterborne Releases

Total body

Thyroid

Bone

GI tract

Liver

(a) Operated by Battelle Memorial Institute for the Department of Energy.

(b) As used in this report, dose commitment describes the total-body or specified organ dose equivalent in rem ( 1 rem $=0.01$ sievert) received over 50 years from intake during the year in which radioactive materials were released into the environment from the power plants. 
TABLE 2. Pathways Considered in This Study

Pathways for

Airborne Releases

Air submersion

Ground irradiation

Inhalation

Ingestion of food crops

and animal product,s
Pathways for Waterborne Releases

Ingestion of drinking water

Ingestion of fish and invertebrates

The "source terms" used to estimate dose commitments produced from each site were the annual measured releases of radioactive materials as reported to the NRC by the plant operators and subsequently published in an NRC public document (Tichler, et a1. 1991). Doses from carbon-14 generally are not included, because carbon-14 releases from most plants are not measured or reported.

The regional population for which we estimated dose commitments included those persons estimated to be living in a region becween 2 and 80 $\mathrm{km}$ around the reactor sites during 1988. Population distributions were supplied by the NRC's Office of Nuclear Reactor Regulation. Atmospheric transport factors (annual average dilution and annual average deposition) were calculated for the region around each site using appropriate meteorological data supplied by the NRC or the utility. To calculate the doses, we used models approved by the NRC. We incorporated these models into two computer programs to expedite the dose calculations involved for each site.

Site-specific parameters other than releases, meteorology, and population were obtained from environmental statements [both final (FES) and draft (DES)] for the various reactors when available (see Table 3 ). Such parameter values include the total population drinking contaminated water, river flow, dilution flow from the reactors (for sites not on rivers), fish and invertebrate harvest for the region, and dilution factors for drinking water and aquatic foods. In those cases in which site-specific data are not readily available and the particular pathway is not expected to result in a large dose, conservative assumptions have been used to estimate doses. The use of more realistic data should result in lower dose estimates in most cases.

The reactors included in this study, their type, licensed therma? power rating and net electrical output for 1988 are listed in Table 4. Populations at risk and the dose commitments derived in the study are also shown in this table. 


\section{TABLE 3. Environmental Statements for Power Plants Included in This Study}

\begin{tabular}{|c|c|}
\hline $\begin{array}{l}\text { Site } \\
\text { number }\end{array}$ & Reactor site \\
\hline 1 & Big Rock Point \\
\hline 2 & Browns ferry \\
\hline 3 & cooper \\
\hline 4 & Dresden \\
\hline 5 & Beaver valley \\
\hline 6 & Humboldt Bay \\
\hline 7 & Lacrosse \\
\hline 8 & millstone Point \\
\hline 9 & mont Icello \\
\hline 10 & Nine mile Point \\
\hline 11 & oyster creek \\
\hline 12 & Peach sottom \\
\hline 13 & Pllgrim \\
\hline 14 & quad cis es \\
\hline 15 & vermont vinnkee \\
\hline 16 & st. Lucle \\
\hline 17 & Brunswick \\
\hline 18 & Ouane Arnold \\
\hline 19 & 1. A. Fitzpatrlck \\
\hline 20 & E. 1. Hatch \\
\hline 21 & Arkansas one \\
\hline 22 & $\begin{array}{l}\text { Connectlicut Yankee } \\
\text { (HaJdam Neck) }\end{array}$ \\
\hline 23 & fort Calhoun \\
\hline 24 & H. B. Robinson \\
\hline 25 & Indian Point \\
\hline 26 & Salew \\
\hline 27 & Kewaunee \\
\hline 28 & malne Yankee \\
\hline 29 & Oconee \\
\hline 30 & Pall sades \\
\hline 31 & Point Beach \\
\hline 32 & Pralfie Island \\
\hline 33 & R. E. CInna \\
\hline 34 & San onofre \\
\hline 35 & Surry \\
\hline 36 & Three mile island \\
\hline 37 & Turkey point \\
\hline 38 & Yankee Rowa \\
\hline 39 & Zlon \\
\hline 40 & Calvert $\mathrm{Cl}$ itls \\
\hline 41 & cook \\
\hline 42 & Trolan \\
\hline 43 & Rancho seco \\
\hline 44 & Crystal River \\
\hline 43 & Davis-Besse \\
\hline 46 & J. M. Farley \\
\hline 47 & North Anna \\
\hline 48 & sequoyah \\
\hline 49 & acculre \\
\hline 30 & Lasalle \\
\hline 51 & Sumer \\
\hline 52 & susquehanna \\
\hline 53 & Grand culf \\
\hline 54 & Callaway \\
\hline 35 & Limerlek \\
\hline 56 & Diabio Canyon \\
\hline 57 & $m p-2$ \\
\hline 38 & Palo verde \\
\hline 59 & Byron \\
\hline 60 & watertord \\
\hline 61 & wolf Creek \\
\hline 62 & Catawba \\
\hline 63 & Fermi \\
\hline 64 & Shor eha: \\
\hline 65 & Hope Creek \\
\hline 66 & Persy \\
\hline 67 & River Bend \\
\hline 68 & Braldwood \\
\hline 69 & Cintion \\
\hline 70 & Harris \\
\hline $\begin{array}{l}71 \\
72\end{array}$ & $\begin{array}{l}\text { vogtle } \\
\text { South Texas Project }\end{array}$ \\
\hline 12 & sulit teas riofech \\
\hline
\end{tabular}

\begin{tabular}{|c|c|c|c|}
\hline & & Jul & 71 \\
\hline $50-298$ & & feb & 73 \\
\hline 30-237. & $50-249$ & Nov & 73 \\
\hline $50-334$ & & jui & 73 \\
\hline $50-409$ & & Jun & 76 \\
\hline $50-243$. & $50-336$ & jun & 73 \\
\hline $50-263$ & & Nov & 72 \\
\hline $50-220$ & & $\operatorname{Jan}$ & 74 \\
\hline $50-219$ & & Dec & 74 \\
\hline 50.277. & $50-278$ & ADr & 73 \\
\hline $50-293$ & & may & 72 \\
\hline $30-254$. & $50-265$ & sep & 72 \\
\hline $50-271$ & & Jul & 72 \\
\hline 50.334 & & Jun & 73 \\
\hline $50-324$. & $50-325$ & jun & 73 \\
\hline 50.331 & & Mar & 73 \\
\hline $50-333$ & & mar & 73 \\
\hline $50-321$ & & OCt & 72 \\
\hline $50-313$. & $50-368$ & feb & 73 \\
\hline $50-213$ & & oct & 73 \\
\hline $50-285$ & & dug & 72 \\
\hline 50-261 & & ADr & 74 \\
\hline $50-247$ & & Sep & 72 \\
\hline 50-272. & $50-311$ & $A D r$ & 73 \\
\hline $50-305$ & & Dec & 72 \\
\hline $50-309$ & & Jul & 72 \\
\hline 50-269, & $50-270 \cdot 50-287$ & Mar & 72 \\
\hline 50.255 & & Jun & 72 \\
\hline 50-266. & $30-30:$ & $\operatorname{may}$ & 72 \\
\hline 50-282. & $50-306$ & may & 73 \\
\hline $50-244$ & & DeC & 73 \\
\hline $50-206$ & & oct & 73 \\
\hline $50-281$ & & Jun & 72 \\
\hline $50-289$ & & DeC & 72 \\
\hline 50.250 & $50-251$ & feb & 72 \\
\hline 50-293. & $50-304$ & DeC & 72 \\
\hline $50-317$ & & $A D r$ & 73 \\
\hline 50.315 & & Aug & 73 \\
\hline $50-344$ & & Jan & 73 \\
\hline $50-312$ & & mar & 73 \\
\hline $50 \cdot 302$ & & may & 73 \\
\hline $50-346$ & & mar & 73 \\
\hline $50-348$. & $50-364$ & Jun & 72 \\
\hline $50-338$. & $50-339$ & ADr & 73 \\
\hline 50-327. & $50-328$ & $F t b$ & 74 \\
\hline 50.369 , & $50-370$ & $A D P$ & 76 \\
\hline 50.373 & $50 \cdot 374$ & Nov & 78 \\
\hline $50 \cdot 395$ & & Jan & 73 \\
\hline $5 C-387$. & $50-388$ & jun & 73 \\
\hline 3(1)-416. & $.50-417$ & Aug & 73 \\
\hline $50-483$. & $50-486$ & mar & 75 \\
\hline $50-352$. & . $50-353$ & Nov & 73 \\
\hline $50-275$, & $50-323$ & may & 73 \\
\hline $50 \cdot 397$ & & Dec & 72 \\
\hline $50-528$ & thru 30.530 & sep & 75 \\
\hline $50-454$. & 50.455 & jul & 74 \\
\hline $50-382$ & & mar & 73 \\
\hline $50-482$ & & jun & 182 \\
\hline $50-413$ & . $30-414$ & Dec & 7 \\
\hline $50-341$ & & JuI & 72 \\
\hline $50-322$ & & Sep & 72 \\
\hline $50-354$. & $50-355$ & feb & 74 \\
\hline $30-440$. & .50 .441 & $A D P$ & 74 \\
\hline 30.458. & $50-459$ & Sep & 74 \\
\hline 50.456. & $.50-457$ & mar & 74 \\
\hline $50-461$. & $50-452$ & oct & 74 \\
\hline $30-400$ & thru $50-403$ & mar & 74 \\
\hline 30.424. & $50-423$ & mar & 74 \\
\hline $50-498$. & $50-499$ & mar & 72 \\
\hline
\end{tabular}

ES ${ }^{\text {(a) }}$ not avallable ES Published by Tenn. valley Authority Draft ES

Shut down - excluded from thls survey Draft ES

Draft ES sep 72

act 73

ug 72

74

Sep 72

72

Jul 72

ar 72

May 72

may 73

Dec 73

Oct 73

un 72

feb 72

FES of Surry 2 used

Draft ES

ES not avallable

Draft ES

(a) Environmental statement

(b) Site added for 1988 
TABLE 4. Reactor Characteristics and Population Total-Body

Dose Commitments, 1988

\begin{tabular}{|c|c|c|c|c|c|c|c|c|c|}
\hline \multirow[b]{2}{*}{ slte } & \multirow[b]{2}{*}{ Unit } & \multirow[b]{2}{*}{ Iype } & \multirow{2}{*}{$\begin{array}{c}\text { L lcensed } \\
\text { Thermal } \\
\text { Powef } \\
(\text { iw) } \\
\end{array}$} & \multirow{2}{*}{$\begin{array}{r}\text { Electric } \\
\text { Energy } \\
\text { ceneralion } \\
1988 \text { (a) } \\
\text { (Tw.hr) } \\
\end{array}$} & \multicolumn{3}{|c|}{$\begin{array}{c}\text { Population Dose Comltwent } \\
\text { (person-rem) }\end{array}$} & \multirow{2}{*}{$\begin{array}{l}\text { Population } \\
\text { at RIsk } \\
\end{array}$} & \multirow{2}{*}{$\begin{array}{c}\text { Average } \\
\text { Indiyidual } \\
\text { rotal-body } \\
\text { Dose } \\
\text { comitment } \\
\text { (miem) } \\
\end{array}$} \\
\hline & & & & & Llquid & Alr (C) & Total & & \\
\hline $\begin{array}{l}\text { Arkansas one } \\
\text { Arkansas one }\end{array}$ & $\stackrel{1}{2}^{2}$ TOTAL & $\begin{array}{l}\text { PWR } \\
\text { PWR }\end{array}$ & $\begin{array}{l}2568 \\
2815 \\
3303\end{array}$ & $\begin{array}{l}3.95 \\
4.95 \\
8.90\end{array}$ & 1.0 & 0.034 & 1.0 & $1.9 f 5$ & $5.4 E-3$ \\
\hline $\begin{array}{c}\text { Beaver valley } \\
\text { Beaver valley }\end{array}$ & $2^{1}$ TOTAL & $\begin{array}{l}\text { PWR } \\
\text { PWR }\end{array}$ & $\begin{array}{l}2660 \\
2660 \\
7980\end{array}$ & $\begin{array}{r}4.98 \\
6.48 \\
11.46\end{array}$ & 0.046 & 0.085 & 0.13 & $3.6 \mathrm{E} 6$ & $3.6 \mathrm{E}-5$ \\
\hline B Ig Rock Polnt (b) 1 & 1 & BwR & 240 & 0.384 & 0.75 & 0.10 & 0.85 & $1.7 E 5$ & $5 \cdot 1 E-3$ \\
\hline B a a I dwood & $2^{1}$ TOTAL & $\begin{array}{l}\text { PWR } \\
\text { PWR }\end{array}$ & $\begin{array}{l}3411 \\
3411 \\
6822\end{array}$ & $\begin{array}{l}3.42 \\
1.35 \\
4.77\end{array}$ & 1.1 & 0.0061 & 1.1 & $4.3 \mathrm{E} 6$ & $2.6 E-4$ \\
\hline $\begin{array}{l}\text { Browns ferty } \\
\text { Browns ferey }\end{array}$ & $\begin{array}{l}1 \\
2 \\
3 \\
\text { TOTAL }\end{array}$ & $\begin{array}{l}\text { BWR } \\
\text { BWR } \\
\text { BWR }\end{array}$ & $\begin{array}{l}3293 \\
3293 \\
3293 \\
9679\end{array}$ & $\begin{array}{l}0 . \\
0: \\
0 \\
0 .\end{array}$ & 1.2 & 0.0011 & 1.2 & $7.5 E 5$ & $1.6 \mathrm{E} \cdot 3$ \\
\hline $\begin{array}{l}\text { Brunswlck } \\
\text { Brunswlck }\end{array}$ & ${ }^{1}$ TOTAL & $\begin{array}{l}\text { BWR } \\
\text { BWR }\end{array}$ & $\begin{array}{l}2436 \\
2436 \\
4872\end{array}$ & $\begin{array}{l}4.45 \\
3.92 \\
8.37\end{array}$ & 0.00 .47 & 0.35 & 0.040 & 2. AES & $1.7 E \cdot 4$ \\
\hline $\begin{array}{l}\text { Byron } \\
\text { Byron }\end{array}$ & $2^{1}$ TOTAL & $\begin{array}{l}\text { PWR } \\
\text { PWR }\end{array}$ & $\begin{array}{l}3411 \\
3411 \\
6822\end{array}$ & $\begin{array}{r}6.29 \\
6.36 \\
12.65\end{array}$ & 0.092 & 0.029 & 0.12 & $9.4 E 5$ & $1.3 E-4$ \\
\hline Callaway & 1 & PWR & 3565 & 8.94 & 0.0001 & 0.0065 & 0.0066 & $3.7 E 5$ & $1.8 E \cdot 5$ \\
\hline $\begin{array}{l}\text { Calvert cliffis } \\
\text { calvert cliffis }\end{array}$ & $2_{\text {TOTAL }}^{1}$ & $\begin{array}{l}\text { PWR } \\
\text { PWR }\end{array}$ & $\begin{array}{l}2700 \\
2700 \\
5400\end{array}$ & $\begin{array}{r}5.16 \\
6.60 \\
11.76\end{array}$ & 1.5 & 0.24 & 1.7 & $2.8 E 6$ & $6.1 E-4$ \\
\hline $\begin{array}{l}\text { Catawba } \\
\text { Catawba }\end{array}$ & $2^{1}$ TOTAL & $\begin{array}{l}\text { PWR } \\
\text { PWR }\end{array}$ & 3411 & $\begin{array}{r}7.63 \\
6.17 \\
13.80\end{array}$ & 1.4 & 0.21 & 1.6 & $1.6 \mathrm{E}$ & $1.0 E-3$ \\
\hline clinton & 1 & BWR & 2694 & 5.86 & 4.0 & 0.0087 & 4.0 & $2.7 E+6$ & $1.5 E-3$ \\
\hline $\begin{array}{l}\text { cook } \\
\text { cook }\end{array}$ & $y^{\prime}$ TOTAL. & $\begin{array}{l}\text { PWR } \\
\text { PWR }\end{array}$ & $\begin{array}{l}3250 \\
3391 \\
6641\end{array}$ & $\begin{array}{l}7.47 \\
2.32 \\
9.79\end{array}$ & 0.21 & 0.0059 & 0.22 & $1.2 \mathrm{E} 6$ & $1.9 E-4$ \\
\hline Cooper & 1 & BWR & 2381 & 4.20 & 0.0068 & 0.0049 & 0.012 & $1.7 \mathrm{ES}$ & $6.8 \mathrm{E}-5$ \\
\hline CrystaI River & 3 & PWR & 2452 & 5.77 & 0.19 & 0.011 & 0.20 & $4.6 \mathrm{E} 5$ & $4 \cdot A E-4$ \\
\hline Davis-Besse & 1 & PWR & 2772 & 1.16 & 0.0018 & 0.016 & 0.018 & $1.8 \mathrm{E} 6$ & $9.9 F \cdot 6$ \\
\hline $\begin{array}{l}\text { Dlabin Canyon } \\
\text { Dlabl.) Canyon }\end{array}$ & $2_{\text {TOTAL }}^{1}$ & $\begin{array}{l}\text { PWR } \\
\text { PWR }\end{array}$ & $\begin{array}{l}3336 \\
3411 \\
6749\end{array}$ & $\begin{array}{r}5.26 \\
6.23 \\
11.19\end{array}$ & 0.0063 & 0.015 & 0.021 & 3. IE 5 & $6.9 E-5$ \\
\hline $\begin{array}{l}\text { Dresden } \\
\text { Dresden }\end{array}$ & ${ }^{\frac{1}{2}}{ }^{3}$ TOTAL & $\begin{array}{l}\text { BWr } \\
\text { BWR } \\
\text { BWR }\end{array}$ & $\begin{array}{r}700 \\
2527 \\
2527 \\
5754\end{array}$ & $\begin{array}{l}0.32 \\
4.32 \\
4.16 \\
0.48\end{array}$ & 0 & 0.11 & 0.11 & $6.5 \mathrm{E} 6$ & $1.7 t-5$ \\
\hline Duane Arnold & 1 & BWR & $16: 38$ & 3.14 & 0 & $0.0 \times 4$ & 0.064 & $5.8 \mathrm{EF}$ & $1.1 E \cdot 4$ \\
\hline $\begin{array}{l}\text { 1. M. Farley } \\
\text { J. M. Farley }\end{array}$ & $i^{1}$ TOTAL & $\begin{array}{l}\text { PWR } \\
\text { PWR }\end{array}$ & $\begin{array}{l}2652 \\
2652 \\
5304\end{array}$ & $\begin{array}{r}5.91 \\
7.17 \\
13.08\end{array}$ & 0.020 & 0.045 & 0.065 & $3.7 \mathrm{ES}$ & $1.7 \mathrm{E}-4$ \\
\hline fermi & 2 & BWR & 3292 & 4.06 & 0.010 & 0.0024 & 0.012 & 4.956 & $2.5 E-6$ \\
\hline J. A. Fitzpateick & 1 & BWR & 2436 & 4.36 & 0.017 & 0.14 & 0.16 & $8.6 \mathrm{E} 5$ & $1.8 \mathrm{E}-4$ \\
\hline Fort Calhoun & 1 & PWR & 1500 & 2.63 & 0.33 & 0.0580 & 0.34 & $7.6 \mathrm{ES}$ & $4.4 E-4$ \\
\hline R. E. Ginna & 1 & PWR & 1520 & 3.53 & 0.16 & 0.073 & 0.23 & $1.2 \mathrm{E} 6$ & $1.9 E-4$ \\
\hline Grand Culf & 1 & BWR & 3833 & 9.39 & 0.0004 & 0.0007 & 0.0011 & $3.4 E 5$ & $3.2 E \cdot 6$ \\
\hline Haddam Neck & 1 & PWR & 1825 & 3.31 & 0.077 & 0.46 & 0.54 & $3.5 E 6$ & $1.5 E-4$ \\
\hline Harris & 1 & PWR & 2775 & 5.33 & 1.7 & 0.10 & 1.8 & 1.416 & $1.3 E-3$ \\
\hline $\begin{array}{l}\text { E. 1. Hatch } \\
\text { E. I. Hatch }\end{array}$ & $2^{1}$ TOTAL & $\begin{array}{l}\text { BWR } \\
\text { BWR }\end{array}$ & $\begin{array}{l}2436 \\
2436 \\
4877\end{array}$ & $\begin{array}{l}4.11 \\
4.25 \\
8.36\end{array}$ & 6.4 & 0.0 .35 & 6.4 & $3.5 \mathrm{E} 5$ & $3.8 E \cdot 2$ \\
\hline Hope Creek & 1 & BWR & 3293 & 6.99 & 0.96 & 0.13 & 1.1 & $4.9 E 6$ & $2.2 E-4$ \\
\hline $\begin{array}{l}\text { Indian polnt } \\
\text { Indian point }\end{array}$ & $3_{\text {TOTAL }}^{1}{ }^{1}$ & $\begin{array}{l}\text { PWR } \\
\text { PWR } \\
\text { PWR }\end{array}$ & $\begin{array}{r}615 \\
2758 \\
2760 \\
6133\end{array}$ & $\begin{array}{r}0 . \\
6.06 \\
6.71 \\
12.77\end{array}$ & 0.092 & 0.34 & 0.43 & $1.6 E 7$ & $2.8 E-5$ \\
\hline Kewaunee & 1 & PwR & 1650 & 3.91 & 1.3 & 0.0022 & 1.3 & $6.3 E 5$ & $2.1 \mathrm{E}-3$ \\
\hline Lacrosse $(b)$ & 1 & BWR & 165 & 0. & 0.41 & 0.0003 & 0.41 & $3.5 E 5$ & $1,2 \mathrm{E}-3$ \\
\hline $\begin{array}{l}\text { Lasalle } \\
\text { Lasalle }\end{array}$ & $\begin{array}{l}1 \\
2 \\
\text { TOTAL. }\end{array}$ & $\begin{array}{l}\text { BWR } \\
\text { BWR }\end{array}$ & $\begin{array}{l}3323 \\
3323 \\
6646\end{array}$ & $\begin{array}{r}5.44 \\
5.66 \\
11.10\end{array}$ & 0 & 0.27 & 0.27 & 1. $1 E 6$ & $2.3 E-4$ \\
\hline Limerlck & & BWR & 3293 & 6.67 & 0 & 0.25 & 0.25 & $6.9 E 6$ & $3.6 \mathrm{E} \cdot 5$ \\
\hline maine Yankee & 1 & PWR & 2630 & 5.02 & 0.0029 & 0.0047 & 0.0076 & $6.3 \mathrm{E} 5$ & $1.2 \mathrm{E}-5$ \\
\hline $\begin{array}{l}\text { mcculte } \\
\text { mccuite }\end{array}$ & ${ }^{1}$ TOTAL & $\begin{array}{l}\text { PWR } \\
\text { PWR }\end{array}$ & $\begin{array}{l}3411 \\
3411 \\
6822\end{array}$ & $\begin{array}{r}7.39 \\
8.05 \\
15.14\end{array}$ & 16. & 0.17 & 16. & $1.8 E 6$ & $9.16=3$ \\
\hline $\begin{array}{l}\text { millstone } \\
\text { Milistone }\end{array}$ & $\begin{array}{l}1 \\
2 \\
3 \\
3 \\
\\
\text { TOTAL }\end{array}$ & $\begin{array}{l}\text { BWR } \\
\text { PWR } \\
\text { PWR }\end{array}$ & $\begin{array}{l}2011 \\
2700 \\
3411 \\
4571\end{array}$ & $\begin{array}{r}5.54 \\
5.73 \\
7.67 \\
18.94\end{array}$ & 0.0027 & 0.22 & 0.22 & $2.6 \mathrm{E} 6$ & $8.5 E-5$ \\
\hline mont icello & 1 & BWR & 1670 & 4.57 & 0 & 0.18 & 0.18 & $2.3 \mathrm{E} 6$ & $8.0 E-5$ \\
\hline $\begin{array}{l}\text { Nine mlle point } \\
\text { Nine mile Point }\end{array}$ & $2_{\text {TOTAL }}^{1}$ & $\begin{array}{l}\text { BWR } \\
\text { BWR }\end{array}$ & $\begin{array}{l}1850 \\
3323 \\
3173\end{array}$ & $\begin{array}{l}0 . \\
2.51 \\
2.51\end{array}$ & 0.21 & 0.0044 & 0.21 & $8.6 \mathrm{E} 5$ & $2.5 \mathrm{E}-4$ \\
\hline
\end{tabular}


TABLE 4. (Contd)

\begin{tabular}{|c|c|c|c|c|c|c|c|c|c|}
\hline \multirow[b]{2}{*}{ slie } & \multirow[b]{2}{*}{ Unit } & \multirow[b]{2}{*}{ Type } & \multirow{2}{*}{$\begin{array}{c}\text { Licensed } \\
\text { Thermal } \\
\text { Power } \\
\text { (Mw) } \\
\end{array}$} & \multirow{2}{*}{$\begin{array}{c}\text { Electrlc } \\
\text { Energy } \\
\text { Ceneration } \\
\text { igso (a) } \\
(T w, h r)(\mathrm{a}) \\
\end{array}$} & \multicolumn{3}{|c|}{$\begin{array}{c}\text { Populat lon Dose comml tment } \\
\text { (persch-(em) }\end{array}$} & \multirow{2}{*}{$\begin{array}{l}\text { Populat lon } \\
\text { at } R \mid s k \\
\end{array}$} & \multirow{2}{*}{$\begin{array}{r}\text { Average } \\
\text { Individua } \\
\text { rotal-Bod } \\
\text { Dose } \\
\text { Commitmen } \\
\text { (mrem) } \\
\end{array}$} \\
\hline & & & & & Llquid & $A \mid r^{(C)}$ & Total & & \\
\hline $\begin{array}{l}\text { iorth Anna } \\
\text { North Anna }\end{array}$ & ${ }^{1}$ TOTAL & $\begin{array}{l}\text { PWR } \\
\text { PWR }\end{array}$ & $\begin{array}{l}2893 \\
2893 \\
5786\end{array}$ & $\begin{array}{r}6.90 \\
7.88 \\
14.78\end{array}$ & 0.01 & 0.026 & 0.036 & 1. $2 \mathrm{E} 6$ & $3.1 E-5$ \\
\hline $\begin{array}{l}\text { oconee } \\
\text { oconee }\end{array}$ & $\begin{array}{ll}1 & \\
2 & \\
3 & \\
& \text { TOTAL }\end{array}$ & $\begin{array}{l}\text { PWR } \\
\text { PWR } \\
\text { PWR }\end{array}$ & $\begin{array}{l}2568 \\
2568 \\
2568 \\
7704\end{array}$ & $\begin{array}{r}7.19 \\
5.54 \\
5.97 \\
18.70\end{array}$ & 3.3 & 0.54 & 3.8 & $9.9 E 5$ & $3.9 E-3$ \\
\hline oyster creek & 1 & BWR & 1930 & 3.54 & 0.20 & 2.0 & 2.2 & $3.6 \mathrm{E} 6$ & $6.0 E-4$ \\
\hline Pallsades & 1 & PWR & 2530 & 3.44 & 0.19 & 0.025 & 0.22 & 1. $1 E 6$ & $2.0 \mathrm{E}-4$ \\
\hline Palo verde & $\begin{array}{ll}1 & \\
2 & \\
3 & \text { TOTAL. }\end{array}$ & $\begin{array}{l}\text { PWR } \\
\text { PWR } \\
\text { PWR }\end{array}$ & $\begin{array}{r}3800 \\
3800 \\
3800 \\
11400\end{array}$ & $\begin{array}{r}6.67 \\
6.75 \\
0.04 \\
13.46\end{array}$ & 0 & 0.20 & 0.20 & 1. $2 \mathrm{ES}$ & $1.7 E-4$ \\
\hline $\begin{array}{l}\text { Peach sottom } \\
\text { Peach Bottom }\end{array}$ & $3^{2}$ TOTAL & $\begin{array}{l}\text { BWR } \\
\text { BWR }\end{array}$ & $\begin{array}{l}3293 \\
3293 \\
6586\end{array}$ & $\begin{array}{l}\mathbf{0} \\
\mathbf{0} \\
\mathbf{0}\end{array}$ & 0.32 & 0.014 & 0.33 & 4. $3 E 5$ & $7.7 E-5$ \\
\hline Perry & 1 & BWR & 3579 & 7.23 & 0.11 & 0.068 & 0.18 & $2.4 E 6$ & $7.5 E \cdot 5$ \\
\hline plIgrIm & 1 & BWR & 1998 & 0 & 0.0014 & 0.0006 & 0.0020 & $4.4 E 6$ & $4.6 E-7$ \\
\hline $\begin{array}{l}\text { Point Beach } \\
\text { Point Beach }\end{array}$ & $i^{1}$ TOTAL & $\begin{array}{l}\text { PWR } \\
\text { PWR }\end{array}$ & $\begin{array}{l}1518 \\
1518 \\
3036\end{array}$ & $\begin{array}{l}3.83 \\
3.72 \\
7.55\end{array}$ & 0.017 & 0.030 & 0.047 & $6.4 E .5$ & $7.4 E \cdot 5$ \\
\hline $\begin{array}{l}\text { Pralrle Island } \\
\text { Pralrie Island }\end{array}$ & $2^{1}$ TOTAL & $\begin{array}{l}\text { PWR } \\
\text { PWR }\end{array}$ & $\begin{array}{l}1650 \\
1650 \\
3300\end{array}$ & $\begin{array}{l}3.82 \\
3.89 \\
7.71\end{array}$ & 0.0031 & 0.077 & 0.080 & $2.3 E 6$ & $3.5 E-5$ \\
\hline $\begin{array}{l}\text { Quad cities } \\
\text { Quad clties }\end{array}$ & $2^{1}$ TOTAL & $\begin{array}{l}\text { BWR } \\
\text { BWR }\end{array}$ & $\begin{array}{l}2511 \\
2511 \\
5022\end{array}$ & $\begin{array}{l}5.66 \\
4.18 \\
9.84\end{array}$ & 0.040 & 0.016 & 0.056 & $7.3 E 5$ & $7.3 E-5$ \\
\hline Rancho seco & 1 & PWR & 2772 & 2.81 & 0.021 & 0.059 & 0.080 & 2.166 & $3.8 \mathrm{E}-5$ \\
\hline RIver Bend & 1 & BWR & 2894 & 7.25 & 0.0067 & 0.0010 & 0.0017 & $7.6 E 5$ & 2. $2 E-6$ \\
\hline H. B. Robinson & 2 & PWR & 2300 & 3.18 & 0.041 & 0.020 & 0.061 & $7.3 E 5$ & 8. $3 E-5$ \\
\hline $\begin{array}{l}\text { St. Lucle } \\
\text { st. Lucle }\end{array}$ & $\begin{array}{ll}1 & \\
2 & \text { TOTAL }\end{array}$ & $\begin{array}{l}\text { PWR } \\
\text { PWR }\end{array}$ & $\begin{array}{l}2560 \\
2700 \\
5260\end{array}$ & $\begin{array}{r}6.25 \\
7.41 \\
13.66\end{array}$ & 0.0002 & 0.36 & 0.36 & $7.2 \mathrm{ES}$ & $5.0 E-4$ \\
\hline $\begin{array}{l}\text { Sa l en } \\
\text { sa lem }\end{array}$ & $\begin{array}{ll}1 & \\
2 & \text { TOTAL. }\end{array}$ & $\begin{array}{l}\text { PWR } \\
\text { PWR }\end{array}$ & $\begin{array}{l}3338 \\
3338 \\
6676\end{array}$ & $\begin{array}{r}7.41 \\
5.97 \\
13.38\end{array}$ & 0.28 & 0.66 & 0.94 & 4. $9 \mathrm{E} 6$ & $1.9 \mathrm{E}-4$ \\
\hline $\begin{array}{l}\text { san onofre } \\
\text { san onotre }\end{array}$ & $\begin{array}{ll}1 & \\
2 & \\
3 & \\
& \text { TOTAL }\end{array}$ & $\begin{array}{l}\text { PWR } \\
\text { PWR } \\
\text { PWR }\end{array}$ & $\begin{array}{l}1347 \\
3410 \\
3390 \\
8147\end{array}$ & $\begin{array}{r}1.37 \\
9.00 \\
6.13 \\
16.50\end{array}$ & 0.023 & 0.48 & 0.50 & $5.5 E 6$ & 9. 1E-5 \\
\hline $\begin{array}{l}\text { Sequoyah } \\
\text { sequoyah }\end{array}$ & $\begin{array}{l}1 \\
2 \\
\end{array}$ TOTAL & $\begin{array}{l}\text { PWR } \\
\text { PWR }\end{array}$ & $\begin{array}{l}2815 \\
2815 \\
5630\end{array}$ & $\begin{array}{l}0.07 \\
3.88 \\
3.95\end{array}$ & 0.26 & 0.019 & 0.28 & B: $8 E 5$ & $3.2 E-4$ \\
\hline Shor eham & 1 & BWR & 121.8 & 0 & 0.0015 & 0 & 0.0015 & 5. $2 E 6$ & $2.9 E-7$ \\
\hline South rexas & 1 & PWR & 3800 & 2.79 & 0.14 & 0.028 & 0.17 & $2.8 E 5$ & 6. $1 E-4$ \\
\hline Summer & 1 & PWR & 2775 & 5.05 & 13. & 0.0070 & 13. & $9.0 E 5$ & $1.4 E-2$ \\
\hline $\begin{array}{l}\text { Surry } \\
\text { surry }\end{array}$ & $\stackrel{1}{2}$ TOTAL $^{2}$ & $\begin{array}{l}\text { PWR } \\
\text { PWR }\end{array}$ & $\begin{array}{l}2441 \\
2441 \\
4882\end{array}$ & $\begin{array}{l}2.69 \\
3.57 \\
6.26\end{array}$ & 0.47 & 0.040 & 0.51 & $1.9 E 6$ & $2.7 E-4$ \\
\hline $\begin{array}{l}\text { Susquehanna } \\
\text { susquehanna }\end{array}$ & $\stackrel{1}{2}$ TOTAL & $\begin{array}{l}\text { BWR } \\
\text { BWR }\end{array}$ & $\begin{array}{l}3293 \\
3293 \\
6586\end{array}$ & $\begin{array}{r}8.41 \\
5.90 \\
14.31\end{array}$ & 0.018 & 0.016 & 0.034 & $1.5 E 6$ & $2.3 \mathrm{E}-5$ \\
\hline $\begin{array}{l}\text { Three mile Island } \\
\text { Three Mile island }\end{array}$ & $\begin{array}{l}1 \\
2 \\
\text { nd TOTAL }\end{array}$ & $\begin{array}{l}\text { PWR } \\
\text { PWR }\end{array}$ & $\begin{array}{l}2535 \\
2772 \\
5307\end{array}$ & $\begin{array}{l}5.47 \\
0.47 \\
5.47\end{array}$ & 0.12 & 0.22 & 0.34 & 2. 1E 6 & $1.6 E-4$ \\
\hline Trojan & 1 & PWR & 3411 & 6.34 & 0.0013 & 0.045 & 0.046 & 1.566 & $3.1 E-5$ \\
\hline $\begin{array}{l}\text { Turkey Point } \\
\text { Turkey Point }\end{array}$ & $\begin{array}{l}3 \\
4 \text { TOTAL }\end{array}$ & $\begin{array}{l}\text { PWR } \\
\text { PWR }\end{array}$ & $\begin{array}{l}2200 \\
2200 \\
4400\end{array}$ & $\begin{array}{l}3.45 \\
3.26 \\
6.71\end{array}$ & 0.045 & 0.14 & 0,18 & $3.0 E 6$ & $6.3 E-5$ \\
\hline vermont vankee & 1 & BWR & 1593 & 4.11 & 0 & 0.059 & 0.059 & $1.4 E 6$ & 4. $1 E \cdot 5$ \\
\hline vogtle & 1 & PWR & 3411 & 6.79 & 0.0029 & 0.018 & 0.021 & $6.2 E 5$ & $3.4 E-5$ \\
\hline Watertord & 3 & PWR & 3390 & 6.54 & 0.063 & 0.48 & 0.54 & 1.856 & $3.0 E-1$ \\
\hline WNP-2 & & BWR & 3323 & 6.00 & 0.0002 & 0.029 & 0.029 & $2.8 E 5$ & $1.1 E-4$ \\
\hline woll creek & 1 & PWR & 3411 & 6.66 & 0.18 & 0.011 & 0.19 & $1.9 E 5$ & $1.06-3$ \\
\hline Yankee Rowe & 1 & PWR & 600 & 1.12 & 0.45 & 0.038 & 0.49 & 1.666 & $3.0 E-4$ \\
\hline $\begin{array}{l}\text { zion } \\
\text { zion }\end{array}$ & $2^{1}$ TOTAL & $\begin{array}{l}\text { PWR } \\
\text { PWR }\end{array}$ & $\begin{array}{l}3250 \\
3250 \\
650 C\end{array}$ & $\begin{array}{r}6.34 \\
6.65 \\
12.99 \\
\end{array}$ & 68 & 0.44 & 7.2 & 7.366 & 1. $0 E-3$ \\
\hline TOTAL FOR ALL SITIS & & & & 511.21 & 65. & 9.6 & 75. & 1.568 & $\ldots$ \\
\hline Arlthmetic mean & & & & 7.2 & 0.92 & 0.14 & 1.1 & 2. $1 f 6$ & $1.1 E \cdot 3$ \\
\hline ceometric mean & & & & 0.80 & 0.086 & 0.038 & 0.18 & 1.266 & $1.5 E-4$ \\
\hline
\end{tabular}

(a) $1 \mathrm{Tw}-\mathrm{hr}=3.6 \mathrm{t} 15$ loules.

(b) Does not have charcoal delay beds in the gaseous effluent line from alr elector 


\section{Site-Dependent Parameters}

In the Site Summaries section, the location (including latitude and longitude) for each reactor site and the estimated 1988 population within 2 to $80 \mathrm{~km}$ around each site is given. In addition, the locations of major metropolitan centers within $80 \mathrm{~km}$ are listed along with treir 1988 extrapolated populations. The populations of the Standard Metropolitan Statistical Areas (SMSA) are given where applicable. Next, the site-specific data pertinent to the airborne pathways are specified. The average production rates of vegetable crops and animal products are given for the area within an $80-\mathrm{km}$ radius based upon the statewide average. This production has been reduced for sites on lakes and seacoasts to account for the presence of the body of water. An animal grazing factor is estimated for each site location. This factor accounts for the fraction of the year during which grazing animals such as milk cows and beef cattle graze on fresh pasture in the region around the site. After average production rates are given, the period of record and the percent data recovery of the meteorological data used in calculating diffusion factors are indicated.

Various site-dependent factors associated with the waterborne pathways are presented next. For lake and ucean sites, we used the average dilution of plant effluents for the year 1988 specified by Tichler, et a1. (1991). For river sites, the average annual river flow is tabulated. This flow was used in place of a dilution flow from the plant to account for dilutions of liquid releases at the locations of probable intake of drinking water and aquatic food catch. Any exceptions to this scheme have been footnoted. Next is shown the estimated 1988 population utilizing drinking water drawn from supplies containing diluted effluents from the site. These are shown with an estimated dilution factor where applicable. Fish and invertebrate catch data taken from the respective plant environmental statement, when available (see Table 3), are listed next, along with estimated dilution factors for the lake and ocean sites. When site-specific fish and invertebrate catch data were not available, the generic consumption rates were used for the particular site. Sites on salt water were assumed to contribute no dose from drinking water. Any invertebrate catch from fresh water was not considered. 


\section{RESULTS}

This report consists of a summary of values used for site-specific parameters at each site, as explained above, and the results of population dose commitment calculations. The population dose commitments are presented in two tables facing the page summarizing site-specific parameters for that site. These tables include both waterborne and airborne pathway dose commitments for the several organs of reference for each age group investigated. They also include the dose to the whole population, which includes all age groups. The airborne population dose commitments for each of 160 segments $(a)$ partitioning the region around the site were divided by the population residing within that segment to derive an average individual dose for that segment. These doses are summarized as a histogram showing percent of the population receiving a given dose level for each site. The fractional population dose from the waterborne pathway was not determined in this manner, because the NRC does not at present take into account the location of individuals exposed via this pathway, except those exposed through ingestion of drinking water.

Population dose commitments estimated for both the waterborne pathways and airborne pathways varied widely over the sites studied. The total dose commitments (from both pathways) varied from a high of i6 to a low of 0.0011 person-rem for plants in operation during the whole year. The arithmetic mean for the total dose from liquid pathways (0.92 person-rem) and airborne pathways (0.14 person-rem) was 1.1 person-rem (see Table 4).

As in past years, most of the plants accounted for less than a personrem to their surrounding population from plant releases of radio- nuclides into liquid effluent streams. Only two sites had waterborne releases that resulted in population doses exceeding 10 person-rem. The sites were McGuire with 16 person-rem and Summer with 13. These waterborne doses were due almost totally to tritium via the drinking water pathway in the case of McGuire and cesium from fish consumption in the case of Summer.

The doses from radionuclide releases from plant vents and/or stacks into the atmosphere also accounted for less than a person-rem for most sites. Only one site had airborne releases resulting in 1 or more personrem. The site was Oyster creek with 2 person-rem. This airborne dose was the result of krypton-88 and Xenon-135.

The total population dose commitments from all sites for 1988 were estimated to be 65 person-rem via waterborne pathways and 10 person-rem via airborne pathways for a total of 75 person-rem (see Table 4).

(a) See Appendix for definition of segments. 
Figure 1 is a histogram for all sites taken together. We can see from this plot that about $97 \%$ of the total population at risk from airborne releases (140 million) would each receive a dose commitment of less than 3 $\times 10^{-4}$ mrem. We can see further that about $9 \%$ receive a dose which is less than $1 \times 10^{-6}$ mrem. However, no attempt was made in this study to estimate the maximum dose commitment received by any one individual from the radionuclides released at any of the sites. However, licensee calculation of doses to the maximally exposed individual at some sites indicated values of up to approximately 100 times average individual doses (on the order of a few millirem per year).

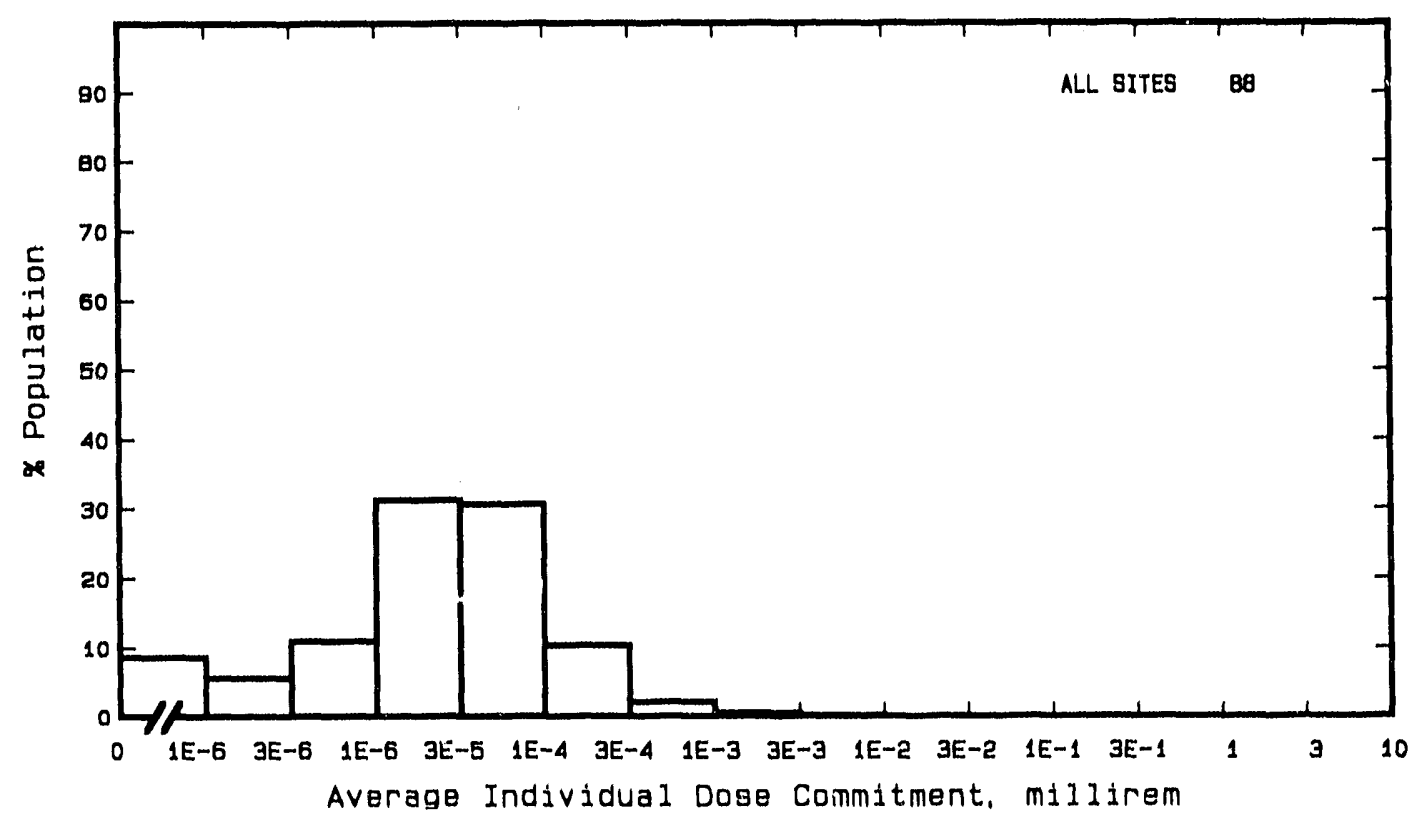

FIGURE 1. Fraction of Total Population Receiving Various Average Individual Total-Body Dose Commitments for Al1 Sites

Figure 2 shows graphically by "Box-and-Whisker Plots" the wide range of the airborne population dose commitments for the reactor sites. The median, upper and lower quartiles and upper and lower octiles for the distribution of doses calculated for each of 160 segments are indicated for each site. The upper and lower octiles are shown as the horizontal bars on the "whiskers;" the upper and lower quartiles are indicated as the top and bottom of the "box." The median is indicated as the horizontal line inside the box. No airborne releases were reported for the Shoreham site for 1988; therefore, none are plotted. 


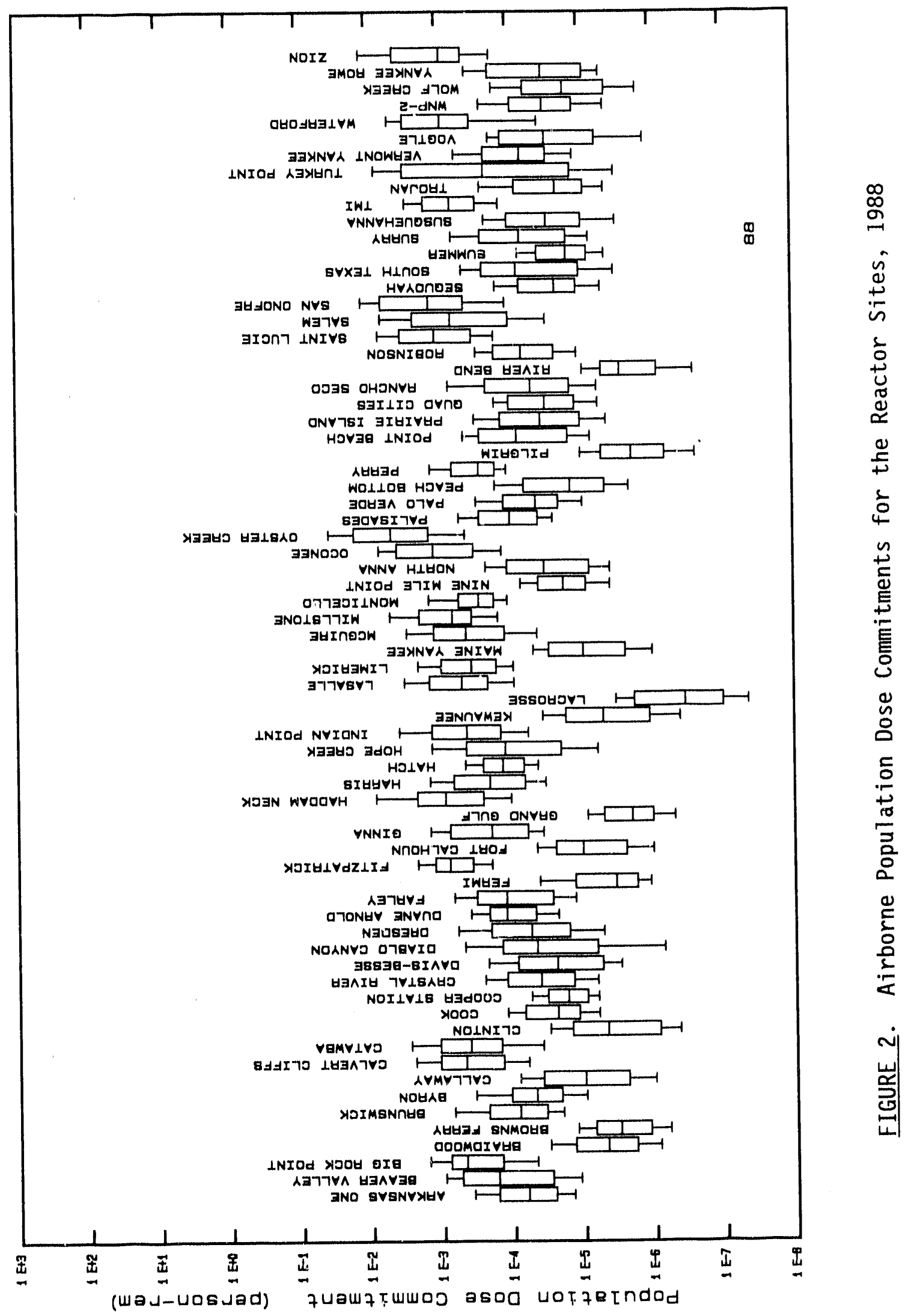


We should point out here, however, that the doses estimated in this study are extremely low compared to an average annual background dose of approximately $100 \mathrm{mrem}$ (neglecting radon daughters). We have compared dose commitments calculated in this study with annual background. However, this comparison is not quite exact, since these dose commitments are those total-body doses received from the year's effluent release over 50 years of a person's lifetime. However, most of the dose commitment calculated here is delivered in the first year, so the comparison is reasonably valid.

For comparison purposes, the doses in the site summary tables are given to two significant figures; however, the data and models used to calculate the doses limit their accuracy to at most one significant figure. 


\section{SITE COMPARISONS}

Table 5 shows the dose commitments for the past 14 years along with the energy produced in that year. The last column shows the cost/benefit for commercial nuclear power as a ratio of population dose to generated power. Except for 1979 (the year of the TMI-2 accident) this ratio has been tending downward even though the population around many of the sites has been rising.

TABLE 5. Comparison of Annual Population Dose Commitments and Energy Output for the Past 14 Years

\begin{tabular}{|c|c|c|c|c|c|}
\hline \multirow{2}{*}{$\begin{array}{l}\text { Year } \\
1975 \\
1976 \\
1977 \\
1978 \\
1979\end{array}$} & $\begin{array}{r}D C \\
\text { Liquid } \\
\end{array}$ & $\begin{array}{l}\text { Commi } \\
\text { Air } \\
\text { Soli-r }\end{array}$ & $\begin{array}{l}\text { ts } \\
\text { Total }\end{array}$ & $\begin{array}{l}\text { Energy } \\
\text { Output } \\
(T W-h r) \\
\end{array}$ & $\begin{array}{l}\text { Cost/Benefit } \\
\text { (person-rem } \\
\frac{T W-h r)}{}\end{array}$ \\
\hline & $\begin{array}{r}76 \\
82 \\
160 \\
110 \\
2.20\end{array}$ & $\begin{array}{r}1300 \\
390 \\
540 \\
530 \\
1600\end{array}$ & $\begin{array}{r}1300 \\
470 \\
700 \\
640 \\
1800\end{array}$ & $\begin{array}{l}170 \\
180 \\
250 \\
270 \\
250\end{array}$ & $\begin{array}{l}7.6 \\
2.5 \\
2.8 \\
2.3 \\
7.2\end{array}$ \\
\hline $\begin{array}{l}1980 \\
1981 \\
1982 \\
1983 \\
1984\end{array}$ & $\begin{array}{r}120 \\
87 \\
50 \\
95 \\
160\end{array}$ & $\begin{array}{r}57 \\
63 \\
87 \\
76 \\
120\end{array}$ & $\begin{array}{l}180 \\
150 \\
140 \\
170 \\
280\end{array}$ & $\begin{array}{l}250 \\
280 \\
270 \\
280 \\
320\end{array}$ & $\begin{array}{l}0.13 \\
0.54 \\
0.51 \\
0.60 \\
0.87\end{array}$ \\
\hline $\begin{array}{l}1985 \\
1986 \\
1987 \\
1988\end{array}$ & $\begin{array}{l}91 \\
71 \\
56 \\
65\end{array}$ & $\begin{array}{r}110 \\
44 \\
22 \\
10\end{array}$ & $\begin{array}{r}200 \\
110 \\
78 \\
75\end{array}$ & $\begin{array}{l}370 \\
410 \\
450 \\
510\end{array}$ & $\begin{array}{l}0.54 \\
0.27 \\
0.17 \\
0.15\end{array}$ \\
\hline
\end{tabular}

The reactor sites are compared in Table 6 as to the total population dose over the years of this study, 1975-1988. The sites were placed within six groupings by person-rem depending on resulting population dose summed over each of the years through 1988:

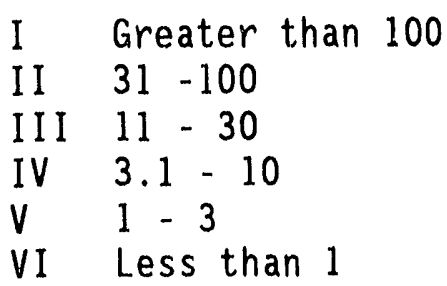


Table 6 shows the sites within the groups along with the reactor manufacturer, year of commercial operation commencement, and the indicated population doses in person-rem. The manufacturer codes are as follows:

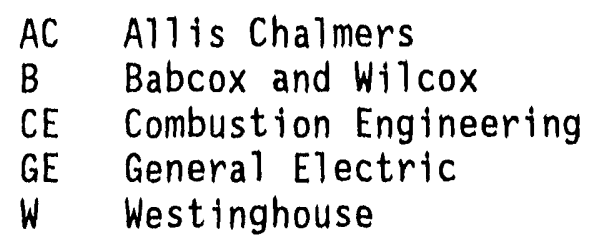

Table 7 shows the average population dose in person-rem for the last three year's. The sites are listed in order of average dose with the high doses toward the top. Breaks in the table are indicated every half order of magnitude: $0.03, .01 .03$, etc.

Table 8 shows the present contributions of all radionuclides contributing over 3\% to the population dose from 1988 effluents. The contributors are shown for each site for both liquid (L) and air (A) pathways. Note that only a few nuclides of those reported by the sites (Tichler, et a). 1991) usually contribute to the dose. 


\section{IABLE 6. Total-Body Population Doses from Nuclear, Power Plant}

Yaar Comerctal

Total person-kem for year

site operation

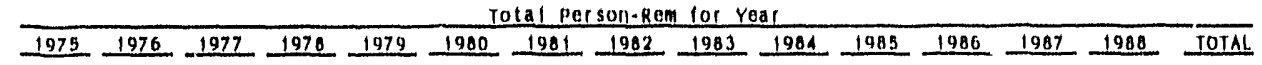

\section{1., 100 person-rem}

oyster creek. OE

Nine Mile Point, OE

zion, $w$

Ocones, a

Peach Bottom, OE

Quad citles.

North Anna.
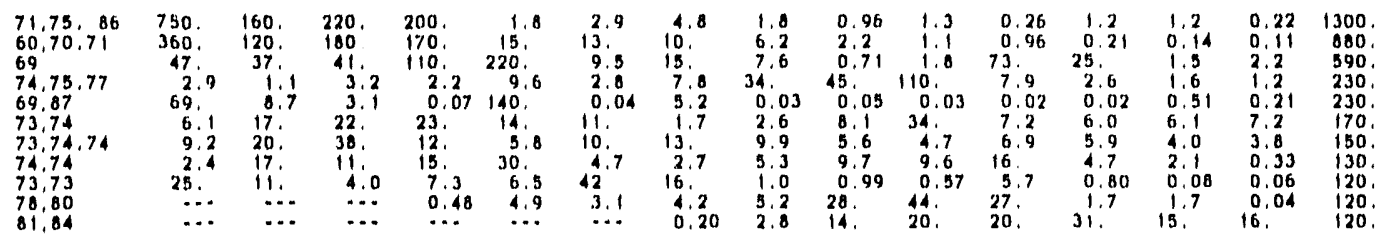

11. 31 - 100 person-rem

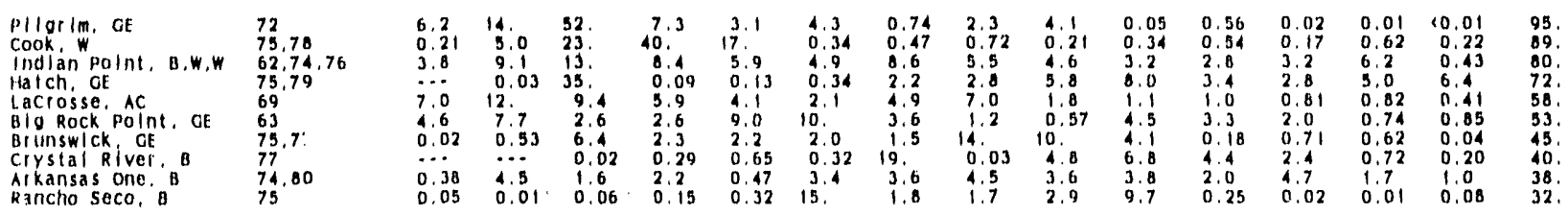

$111.11-30$ person-rem
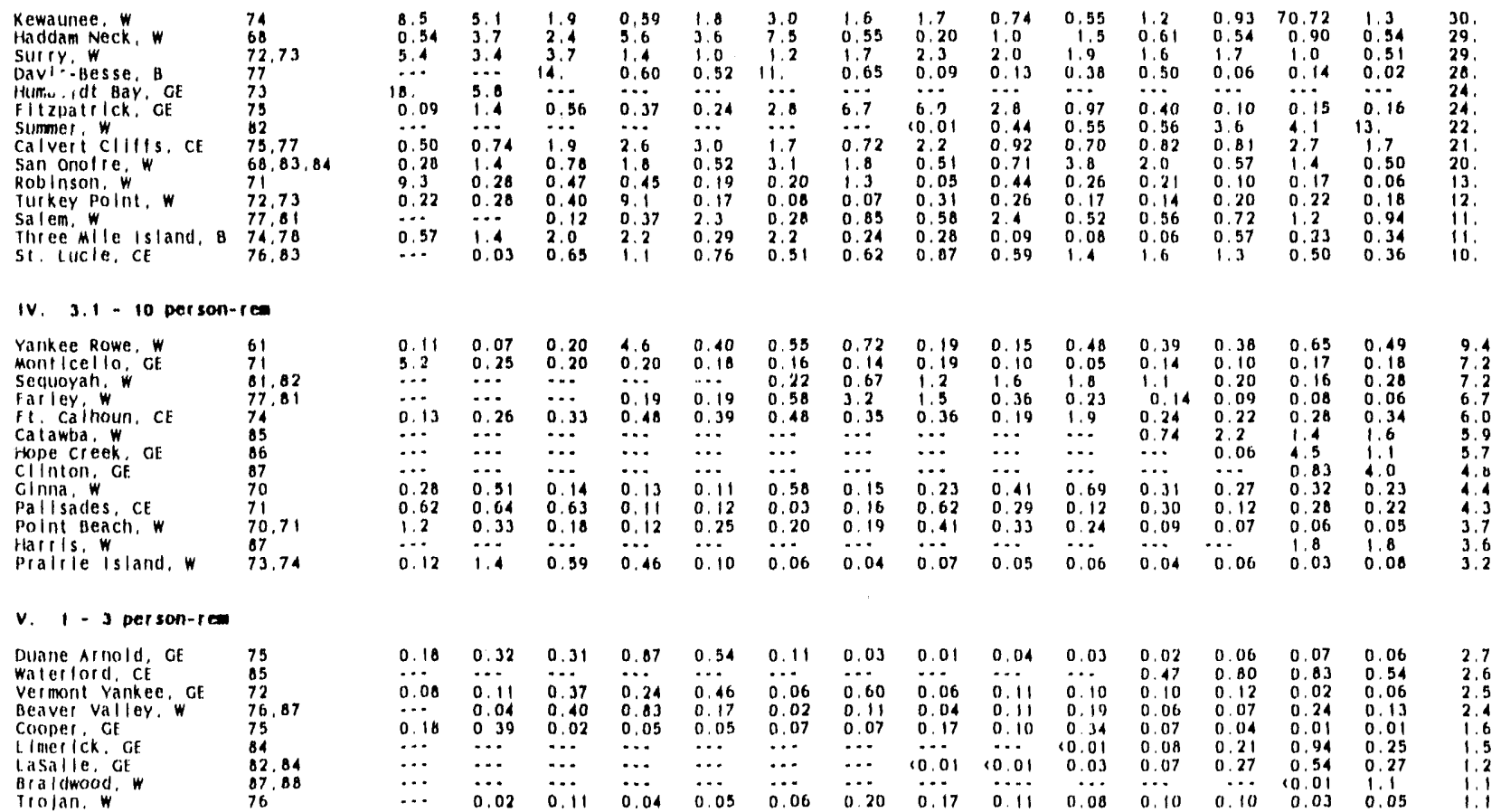

vi. $I$ person-rea
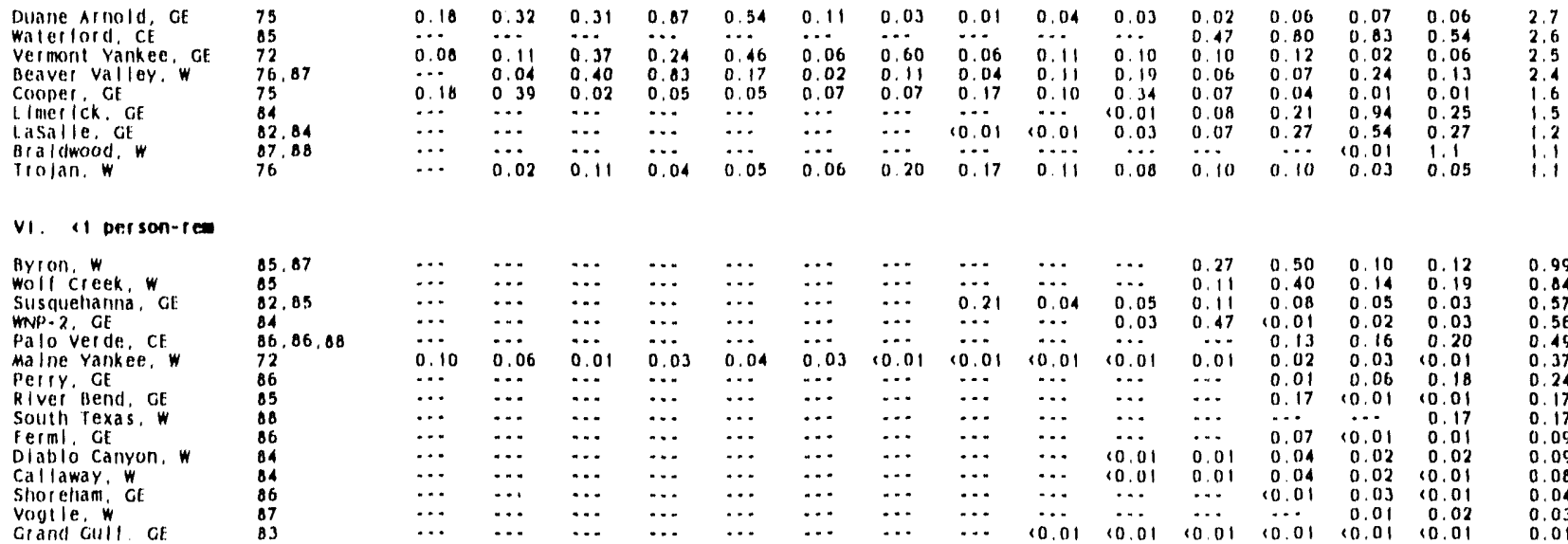

IOIAl.
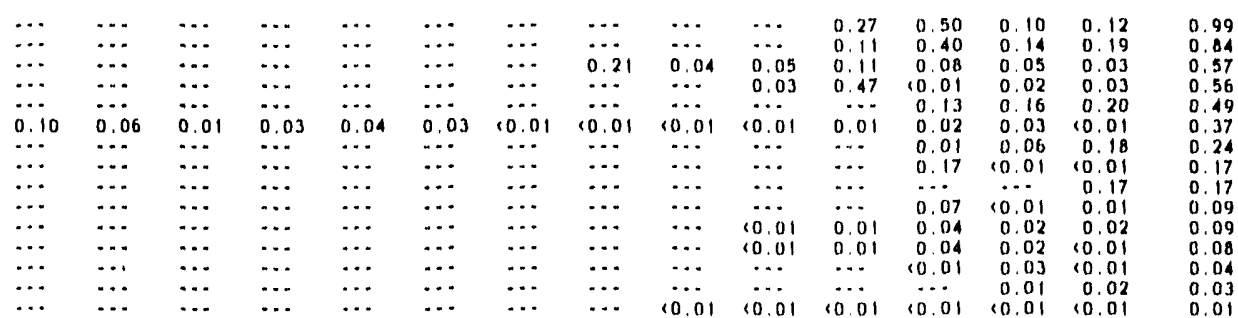

(a) Only the doses from the THI accident, 1979, are excluded.

(i) Does not include doses irom nuclides not reported in releases, such as carbon-14 
Table 7. Average Population Dose for Last Three Years, person-rem

\begin{tabular}{|c|c|c|c|c|}
\hline Site & 1986 & 1087 & 1988 & Average \\
\hline McGuire & 31.10 & 15.17 & 16.17 & 20.81 \\
\hline $\begin{array}{l}\text { Oyster Creek } \\
\text { Summer } \\
\text { Zion } \\
\text { Hatch } \\
\text { Oconee } \\
\text { Indian Pt. }\end{array}$ & $\begin{array}{r}25.00 \\
3.60 \\
6.04 \\
2.75 \\
5.93 \\
3.20\end{array}$ & $\begin{array}{l}1.50 \\
4.11 \\
6.09 \\
4.96 \\
3.97 \\
6.20\end{array}$ & $\begin{array}{r}2.20 \\
13.01 \\
7.24 \\
6.43 \\
3.84 \\
0.43\end{array}$ & $\begin{array}{l}9.57 \\
6.91 \\
6.46 \\
4.71 \\
4.58 \\
3.28\end{array}$ \\
\hline $\begin{array}{l}\text { Arkansas One } \\
\text { Peach Bottom } \\
\text { Hope Creek } \\
\text { Browns Ferry } \\
\text { Calvert Cliffs } \\
\text { Catawba } \\
\text { Clinton } \\
\text { Harris } \\
\text { Big Rock Point } \\
\text { North Anna } \\
\text { Crystal River } \\
\text { Surry }\end{array}$ & $\begin{array}{l}4.73 \\
4.71 \\
0.06 \\
2.64 \\
0.81 \\
2.15 \\
\therefore . . \\
\cdots 1.97 \\
1.97 \\
2.41 \\
1.70\end{array}$ & $\begin{array}{l}1.71 \\
2.07 \\
4.50 \\
1.60 \\
2.66 \\
1.37 \\
0.83 \\
1.78 \\
0.74 \\
1.72 \\
0.72 \\
1.04\end{array}$ & $\begin{array}{l}1.03 \\
0.33 \\
1.09 \\
1.20 \\
1.74 \\
1.61 \\
4.01 \\
1.80 \\
0.85 \\
0.04 \\
0.20 \\
0.51\end{array}$ & $\begin{array}{l}2.49 \\
2.37 \\
1.88 \\
1.81 \\
1.74 \\
1.71 \\
1.61 \\
1.19 \\
1.10 \\
1.14 \\
1.11 \\
1.08\end{array}$ \\
\hline $\begin{array}{l}\text { Kewaunee } \\
\text { Salem } \\
\text { Millstone } \\
\text { San Onofre } \\
\text { Waterford } \\
\text { St. Lucie } \\
\text { LaCrosse } \\
\text { Haddam Neck } \\
\text { Yankee Rowe } \\
\text { Limerick } \\
\text { Brunswick } \\
\text { Three Mile Isl. } \\
\text { Braidwoud } \\
\text { LaSalle } \\
\text { Couk } \\
\text { Quad Cities }\end{array}$ & $\begin{array}{l}0.93 \\
0.72 \\
1.23 \\
0.57 \\
0.80 \\
1.30 \\
0.81 \\
0.54 \\
0.38 \\
0.21 \\
0.71 \\
0.57 \\
0.27 \\
0.17 \\
0.80\end{array}$ & $\begin{array}{l}0.72 \\
1.17 \\
1.20 \\
1.43 \\
0.83 \\
0.50 \\
0.82 \\
0.90 \\
0.65 \\
0.94 \\
0.62 \\
0.23 \\
<0.01 \\
0.54 \\
0.62 \\
0.08\end{array}$ & $\begin{array}{l}1.30 \\
0.94 \\
0.22 \\
0.50 \\
0.54 \\
0.36 \\
0.41 \\
0.54 \\
0.49 \\
0.25 \\
0.04 \\
0.34 \\
1.11 \\
0.27 \\
0.22 \\
0.06\end{array}$ & $\begin{array}{l}0.98 \\
0.94 \\
0.89 \\
0.83 \\
0.72 \\
0.72 \\
0.68 \\
0.66 \\
0.50 \\
0.47 \\
0.46 \\
0.38 \\
0.37 \\
0.36 \\
0.33 \\
0.31\end{array}$ \\
\hline $\begin{array}{l}\text { Ft. Calhoun } \\
\text { Ginna } \\
\text { Nine Mile Point } \\
\text { Holf Creek } \\
\text { Byron } \\
\text { Sequoyah } \\
\text { Pal isades } \\
\text { Turkey Point } \\
\text { Pala Verde } \\
\text { Dresden } \\
\text { Mont icello } \\
\text { Beaver Vall ey } \\
\text { Fitzpatrick } \\
\text { Robinson }\end{array}$ & $\begin{array}{l}0.22 \\
0.27 \\
0.02 \\
0.40 \\
0.50 \\
0.20 \\
0.12 \\
0.20 \\
0.13 \\
0.21 \\
0.10 \\
0.07 \\
0.10 \\
0.10\end{array}$ & $\begin{array}{l}0.28 \\
0.32 \\
0.51 \\
0.14 \\
0.10 \\
0.16 \\
0.28 \\
0.22 \\
0.16 \\
0.14 \\
0.17 \\
0.23 \\
0.15 \\
0.17\end{array}$ & $\begin{array}{l}0.34 \\
0.23 \\
0.21 \\
0.19 \\
0.12 \\
0.28 \\
0.21 \\
0.18 \\
0.20 \\
0.11 \\
0.18 \\
0.13 \\
0.16 \\
0.06\end{array}$ & $\begin{array}{l}0.28 \\
0.28 \\
0.25 \\
0.24 \\
0.24 \\
0.21 \\
0.20 \\
0.20 \\
0.16 \\
0.15 \\
0.15 \\
0.15 \\
0.14 \\
0.11\end{array}$ \\
\hline $\begin{array}{l}\text { Perry } \\
\text { Farley } \\
\text { Davis-Besse } \\
\text { Duane Arnold } \\
\text { Point Beach } \\
\text { Trojan } \\
\text { Vermont Yankee } \\
\text { River Bend } \\
\text { South Texas } \\
\text { Prairie Island } \\
\text { Susquehanna } \\
\text { Rancho Seco }\end{array}$ & $\begin{array}{l}0.00 \\
0.09 \\
0.06 \\
0.06 \\
0.07 \\
0.10 \\
0.12 \\
0.17 \\
0.06 \\
0.06 \\
0.08 \\
0.02\end{array}$ & $\begin{array}{l}0.06 \\
0.08 \\
0.14 \\
0.07 \\
0.06 \\
0.03 \\
0.02 \\
<0.01 \\
0.0 \\
0.03 \\
0.05 \\
0.01\end{array}$ & $\begin{array}{l}0.18 \\
0.06 \\
0.02 \\
0.06 \\
0.05 \\
0.05 \\
0.06 \\
<0.01 \\
0.17 \\
0.08 \\
0.03 \\
0.08\end{array}$ & $\begin{array}{l}0.08 \\
0.08 \\
0.07 \\
0.06 \\
0.06 \\
0.06 \\
0.06 \\
0.06 \\
0.06 \\
0.06 \\
0.06 \\
0.04\end{array}$ \\
\hline $\begin{array}{l}\text { Fermi } \\
\text { Diablo Canyon } \\
\text { Call laway } \\
\text { Maine Yankee } \\
\text { Cooper } \\
\text { WNP-2 } \\
\text { Shoreham } \\
\text { Pilgrim } \\
\text { Vogtle } \\
\text { Grand Gulf }\end{array}$ & $\begin{array}{r}0.07 \\
0.04 \\
0.04 \\
0.02 \\
0.04 \\
<0.01 \\
<0.01 \\
0.02 \\
<0.01\end{array}$ & $\begin{array}{r}<0.01 \\
0.02 \\
0.02 \\
0.03 \\
0.01 \\
0.02 \\
0.03 \\
0.01 \\
0.01 \\
<0.01\end{array}$ & $\begin{array}{r}0.01 \\
0.02 \\
<0.01 \\
<0.01 \\
0.01 \\
0.03 \\
<0.01 \\
<0.01 \\
0.02 \\
<0.01\end{array}$ & $\begin{array}{l}0.03 \\
0.03 \\
0.02 \\
0.02 \\
0.02 \\
0.02 \\
0.01 \\
0.01 \\
0.01 \\
0.01\end{array}$ \\
\hline
\end{tabular}


TABLE 8. Major Radionucilde Contributions to Population Doses from Liquid (L) and Air (A) Pathways

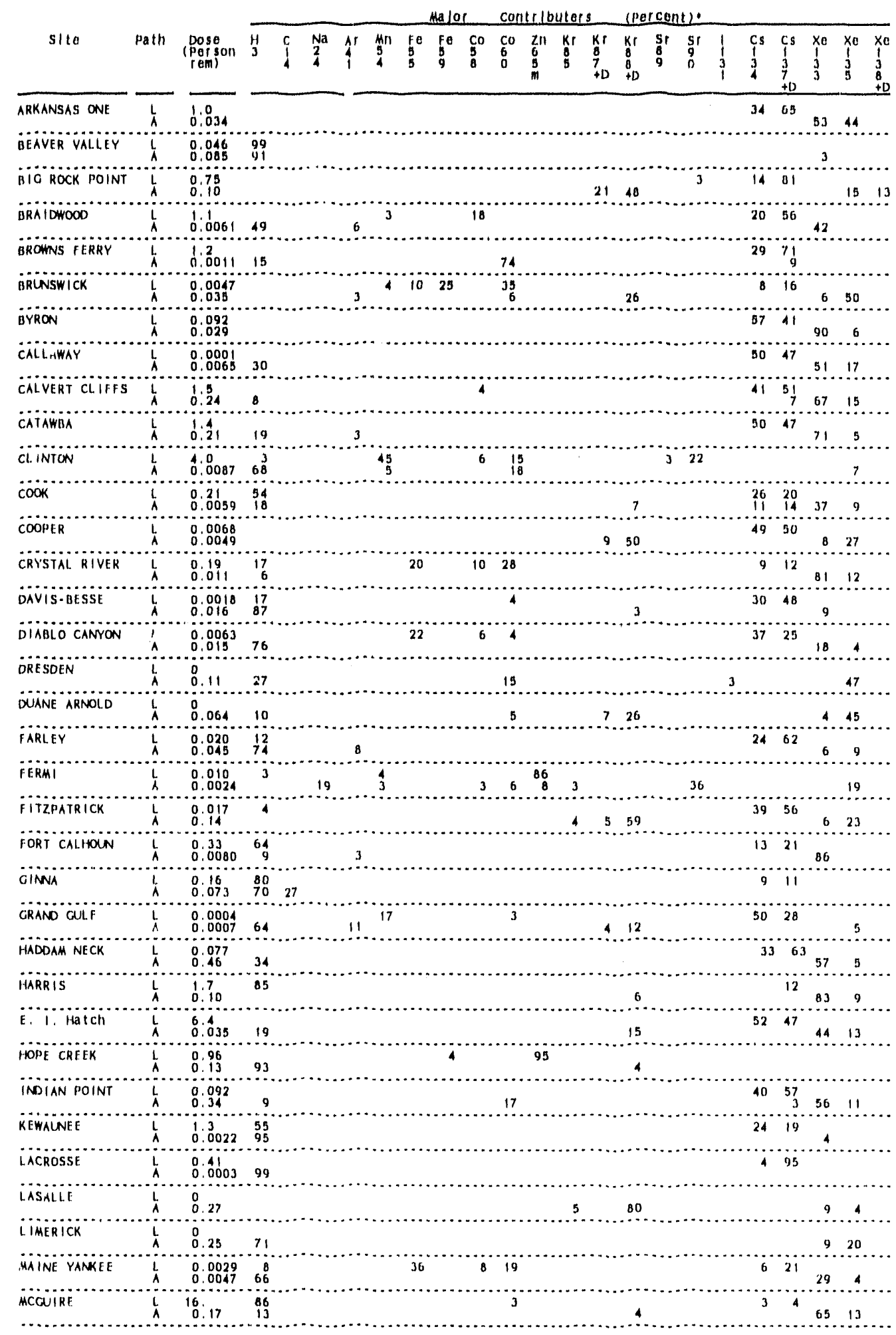


TABLE 8. (Contd)

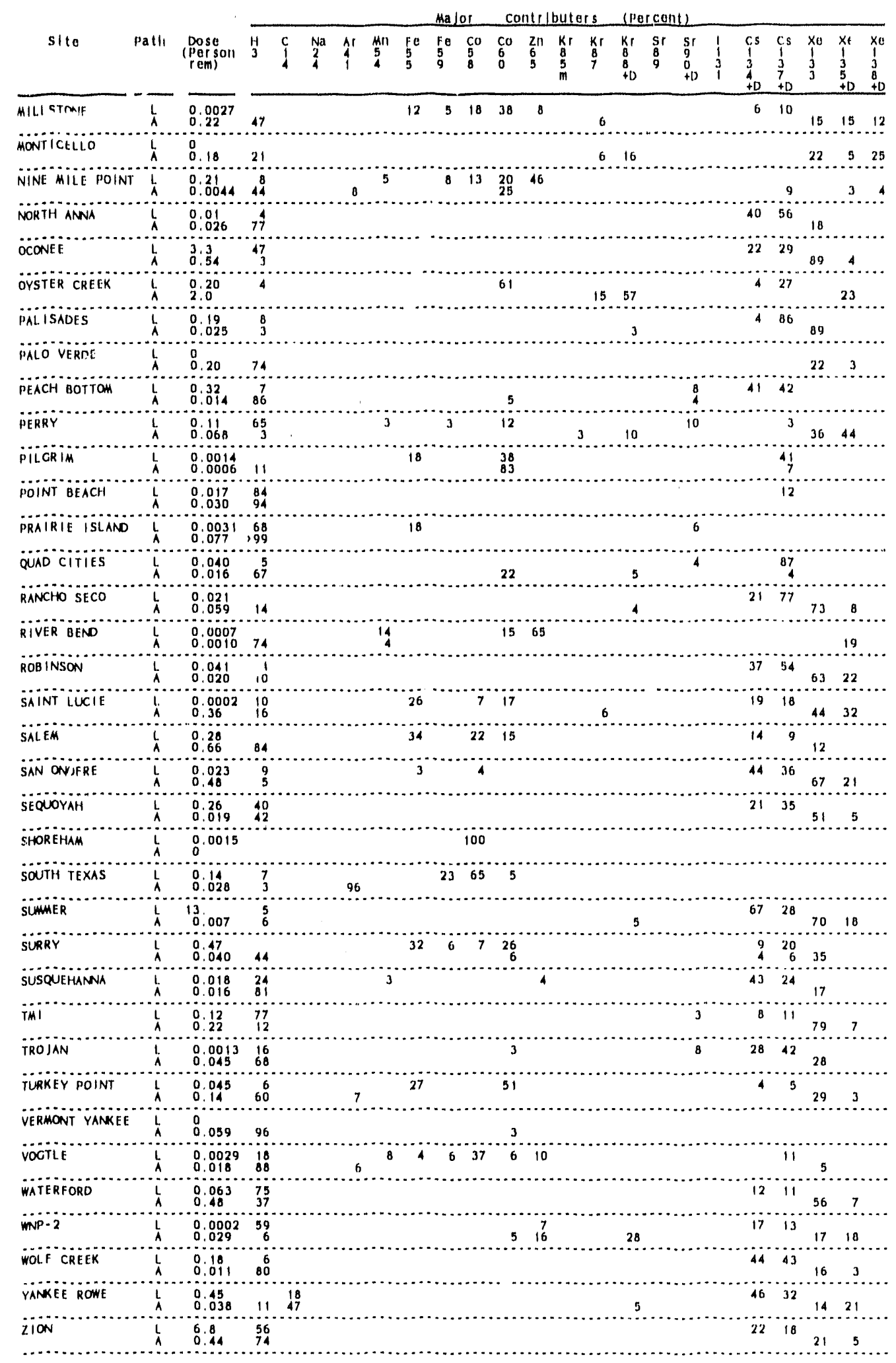

- Contributions less than $3 x$ are not included in table. 
SITE SUMMARIES

1988

2. i 
Site: ARKANSAS ONE

POPE COUNTY, ARKANSAS

Location: N $35.3100^{\circ}$ W $93.2308^{\circ}$

POPULATION DATA

Total Population Within 2-to-80-km Reginn: 1.9E5

Major Metropolitan Centers Within Region:

Center

Russellville

Conway
Population

14,000

20,000
Location

$10 \mathrm{~km} \quad \mathrm{E}$ $76 \mathrm{~km} \quad \mathrm{ESE}$

SITE-SPECIFIC DATA - AIRBORNE PATHWAYS

Average Annual State Production

of Crops and Animal Products

in 80-km Radius Circle

Regional Productivity Factor:

Animal Grazing Factor:

Meteorology Period of Record: 1 JAN 75 - 31 DEC 75 Recovery: 97\%
Veg: $5.8 \mathrm{E} 6$ kilogram

Milk: 4.8E7 liter

Meat: $7.2 \mathrm{E} 7$ kilogram

1

0.7

SITE-SPECIFIC DATA - WATERBORNE PATHWAYS via ARKANSAS RIVER

Average River Flow at Site: $36,000 \mathrm{ft}^{3} / \mathrm{s}$

Drinking Water:

None

Fish:

Edible Harvest: $1.4^{(a)} \mathrm{kg} / \mathrm{yr}$ Dilution Factor: 1

(a) Average individual consumption rates as given in the Final Environmental Statement (FES) (1973) were used in lieu of catch data. The reference to environmental statements here and on succeeding pages may be found in Table 3 . 


\section{POPULATION DOSE-COMMITMENT ESTIMATES AND \\ AVERAGE INDIVIDUAL DOSE-COMMITMENT HISTOGRAM FOR}

ARKANSAS ONE 1 AND 2

Dose Commitments (person-rem) from Waterborne Pathways

Total Body GI-LLI Thyroid Bone Liver

$\begin{array}{llllll}\text { Infant } & 0.0 \mathrm{E}+00 & 0.0 \mathrm{E}+00 & 0.0 \mathrm{E}+00 & 0.0 \mathrm{E}+00 & 0.0 \mathrm{E}+00 \\ \text { Child } & 4.4 \mathrm{E}-02 & 1.8 \mathrm{E}-02 & 8.0 \mathrm{E}-03 & 2.4 \mathrm{E}-01 & 2.6 \mathrm{E}-01 \\ \text { Teen } & 8.4 \mathrm{E}-02 & 3.8 \mathrm{E}-02 & 5.8 \mathrm{E}-03 & 1.4 \mathrm{E}-01 & 2.2 \mathrm{E}-01 \\ \text { Adult } & 9.2 \mathrm{E}-01 & 3.3 \mathrm{E}-01 & 3.8 \mathrm{E}-02 & 8.3 \mathrm{E}-01 & 1.3 \mathrm{E}+00 \\ \text { TOTAL } & 1.0 \mathrm{E}+00 & 3.9 \mathrm{E}-01 & 5.2 \mathrm{E}-02 & 1.2 \mathrm{E}+00 & 1.8 \mathrm{E}+00\end{array}$

Dose Commitments (person-rem) from Airborne Pathways

Total Body GI-LLI Thyroid Bone Liver

$\begin{array}{lllllll}\text { Infant } & 4.9 \mathrm{E}-04 & 4.9 \mathrm{E}-04 & 6.7 \mathrm{E}-04 & 4.8 \mathrm{E}-04 & 4.9 \mathrm{E}-04 & 5.2 \mathrm{E}-04 \\ \text { Child } & 5.5 \mathrm{E}-03 & 5.4 \mathrm{E}-03 & 6.5 \mathrm{E}-03 & 5.3 \mathrm{E}-03 & 5.5 \mathrm{E}-03 & 5.9 \mathrm{E}-03 \\ \text { Teen } & 4.0 \mathrm{E}-03 & 4.0 \mathrm{E}-03 & 4.4 \mathrm{E}-03 & 3.9 \mathrm{E}-03 & 4.0 \mathrm{E}-03 & 4.6 \mathrm{E}-03 \\ \text { Adult } & 2.4 \mathrm{E}-02 & 2.4 \mathrm{E}-02 & 2.6 \mathrm{E}-02 & 2.4 \mathrm{E}-02 & 2.4 \mathrm{E}-02 & 2.6 \mathrm{E}-02 \\ \text { TOTAL } & 3.4 \mathrm{E}-02 & 3.4 \mathrm{E}-02 & 3.7 \mathrm{E}-02 & 3.3 \mathrm{E}-02 & 3.4 \mathrm{E}-02 & 3.7 \mathrm{E}-02\end{array}$

Production/Consumption factors: (a)

Produce: 0.15 Milk: $1.9 \quad$ Meat: 4.6

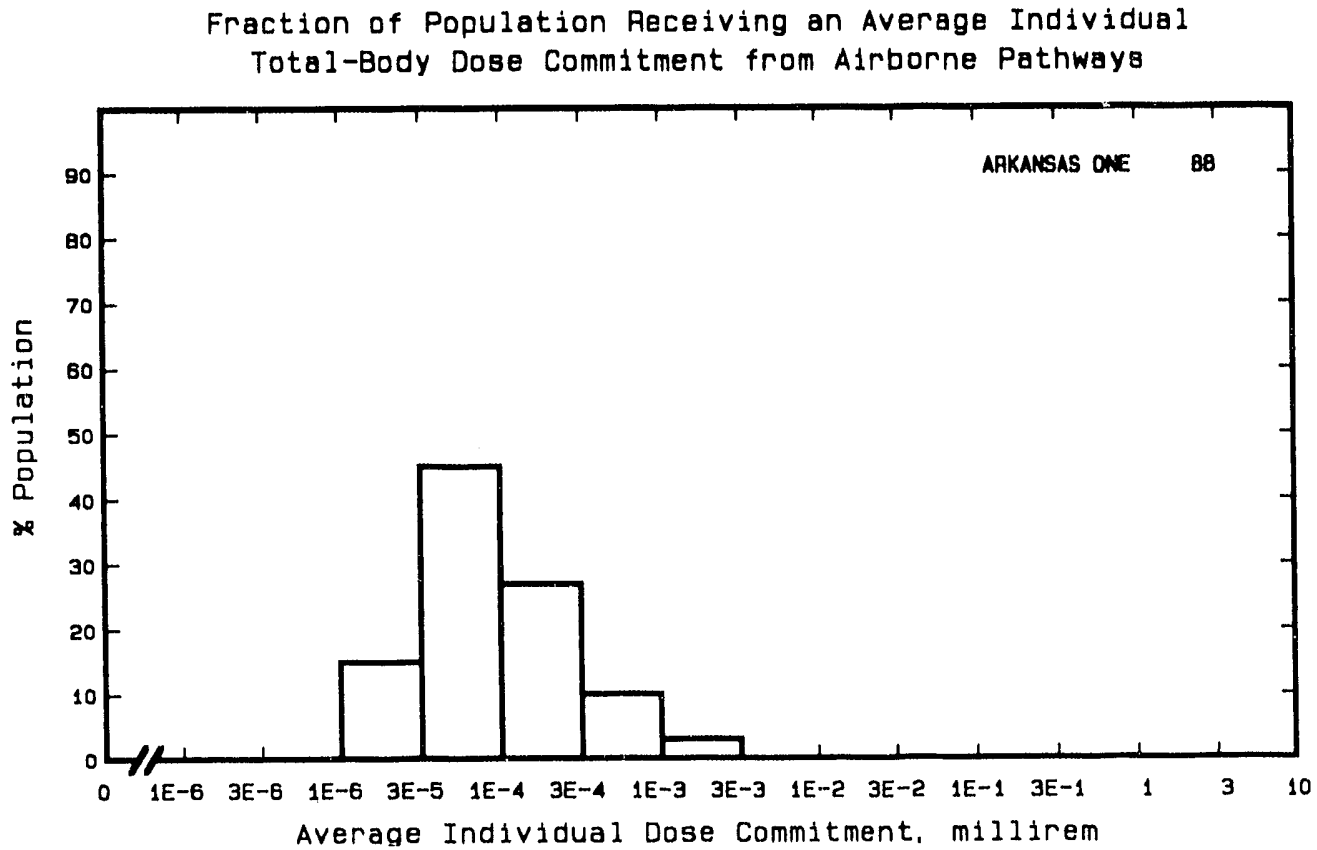

(a) See Appendix A, Page A-5, for Explanation of this ratio. 
Site: BEAVER VALLEY

SHIPPINGPORT, PENNSYLVANIA

Location: N $40.6219^{\circ} \quad$ W $80.4339^{\circ}$

POPULATION DATA

Total Population Within 2-to-80-km Region: 3.7E6

Major Metropolitan Centers Within Region:

Center Population Location

$\begin{array}{lrrr}\text { Pittsburgh SMSA } & 2,100,000 & 42 \mathrm{~km} & \text { ESE } \\ \text { Youngstown-Warren SMSA } & 520,000 & 56 \mathrm{~km} & \text { NNW } \\ \text { Stuebenville-Weirton SMSA } & 160,000 & 33 \mathrm{~km} & \text { SSW } \\ \text { Wheeling SMSA } & 190,000 & 66 \mathrm{~km} & \text { SSW } \\ \text { New Castle } & 35,000 & 43 \mathrm{~km} & \text { N }\end{array}$

SITE-SPECIFIC DATA - AIRBORNE PATHWAYS

Average Annual State Production

of Crops and Animal Products

In $80-\mathrm{km}$ Radius Circle

Regional Productivity Factor:

Animal Grazing Factor:

Meteorology Period of Record: 1 JAN 77 - 31 DEC 77 Recovery: 92\%
Veg: $5.3 \mathrm{E} 7 \mathrm{ki}$ ogram

Milk: 5.3E8 liter

Meat: $5.4 \mathrm{E} 7 \mathrm{kilogram}$

1

0.5

SITE-SPECIFIC DATA - WATERBORNE PATHWAYS via OHIO RIVER

Average River $\mathrm{Flow}$

at Site: $30,000 \mathrm{ft}^{3} / \mathrm{s}$

Drinking Water:

Exposed Population: 6,200

Fish:

Edible Harvest: $410 \mathrm{~kg} / \mathrm{yr}$

Dilution Factor: 1

(a) This factor accounts for the incomplete dilution of plant effluent by river at point of drinking water intake at Midland. 


\section{POPULATION DOSE-COMMITMENT ESTIMATES AND \\ AVERAGE INDIVIDUAL DOSE-COMMITMENT HISTOGRAM FOR \\ BEAVER VALLEY 1 AND 2}

Dose Commitments (person-rem) from Waterborne Pathways

Total Body GI-LLI Thyroid Bone Liver

$\begin{array}{llllll}\text { Infant } & 8.7 \mathrm{E}-04 & 8.7 \mathrm{E}-04 & 9.0 \mathrm{E}-04 & 1.4 \mathrm{E}-05 & 8.9 \mathrm{E}-04 \\ \text { Child } & 9.8 \mathrm{E}-03 & 9.9 \mathrm{E}-03 & 9.9 \mathrm{E}-03 & 1.6 \mathrm{E}-04 & 9.9 \mathrm{E}-03 \\ \text { Teen } & 3.7 \mathrm{E}-03 & 3.9 \mathrm{E}-03 & 3.8 \mathrm{E}-03 & 4.0 \mathrm{E}-05 & 3.8 \mathrm{E}-03 \\ \text { Adult } & 3.2 \mathrm{E}-02 & 3.3 \mathrm{E}-02 & 3.2 \mathrm{E}-02 & 2.5 \mathrm{E}-04 & 3.2 \mathrm{E}-02 \\ \text { TOTAL } & 4.6 \mathrm{E}-02 & 4.8 \mathrm{E}-02 & 4.7 \mathrm{E}-02 & 4.6 \mathrm{E}-04 & 4.7 \mathrm{E}-02\end{array}$

Dose Commitments (person-rem) from Airborne Pathways

Total Body GI-LLI Thyroid Bone Liver Lung

$\begin{array}{lllllll}\text { Infant } & 1.5 \mathrm{E}-03 & 1.4 \mathrm{E}-03 & 5.4 \mathrm{E}-03 & 1.3 \mathrm{E}-04 & 1.5 \mathrm{E}-03 & 1.5 \mathrm{E}-03 \\ \text { Child } & 1.7 \mathrm{E}-02 & 1.7 \mathrm{E}-02 & 3.9 \mathrm{E}-02 & 1.3 \mathrm{E}-03 & 1.7 \mathrm{E}-02 & 1.8 \mathrm{E}-02 \\ \text { Teen } & 1.1 \mathrm{E}-02 & 1.1 \mathrm{E}-02 & 2.0 \mathrm{E}-02 & 8.7 \mathrm{E}-04 & 1.1 \mathrm{E}-02 & 1.2 \mathrm{E}-02 \\ \text { Adult } & 5.6 \mathrm{E}-02 & 5.6 \mathrm{E}-02 & 8.8 \mathrm{E}-02 & 5.0 \mathrm{E}-03 & 5.6 \mathrm{E}-02 & 6.0 \mathrm{E}-02 \\ \text { TOTAL } & 8.5 \mathrm{E}-02 & 8.5 \mathrm{E}-02 & 1.5 \mathrm{E}-01 & 7.3 \mathrm{E}-03 & 8.6 \mathrm{E}-02 & 9.1 \mathrm{E}-02\end{array}$

Production/Consumption factors:

Produce: 0.075 Milk: $1.1 \quad$ Meat: 0.19

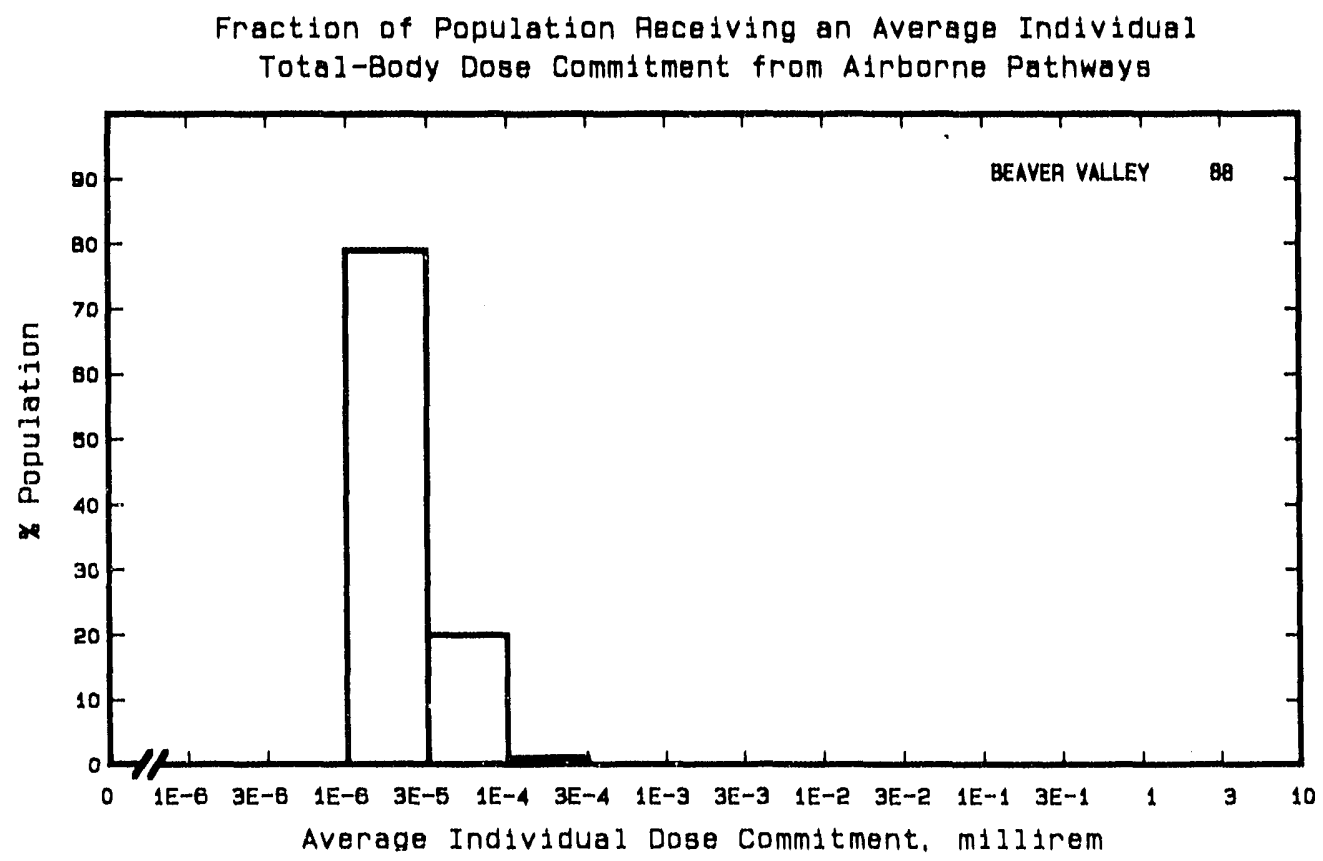


Site: BIG ROCK POINT

CHARLEVOIX COUNTY, MICHIGAN

Location: $\quad N 45.3592^{\circ} \quad$ W $85.1947^{\circ}$

POPULATION DATA

Total Population Within 2-to-80-km Region: 1.7E5

Major Metropolitan Centers Within Region:

Center

Population

Location

Traverse City

Petoskey

16,000

6,100

Cheboygan

5,100

$75 \mathrm{~km} \quad \mathrm{SSW}$

$18 \mathrm{~km} \quad \mathrm{E}$

$65 \mathrm{~km} \quad \mathrm{ENE}$

\section{SITE-SPECIFIC DATA .. AIRBORNE PATHWAYS}

Average Annual State Production

of Crops and Animal Products

In $80-\mathrm{km}$ Radius Circle

Regional Productivity Factor:

Animal Grazing Factor:
Veg: $6.9 E 7$ kilogram

Milk: $2.9 E 8$ itter

Meat: $4.5 E 7$ kilogram

0.5

0.5

Meteorology Period of Record: 9 FEB $61-8$ FEB 63 Recovery: 85\%

Drinking Water:

Fish:

\section{via LAKE MICHIGAN}

Average Dilution Flow

from Plant: $92 \mathrm{ft}^{3} / \mathrm{s}$

Exposed Population: 31,009 (a)

Dilution Factor: $4.9 E-5(b)$

Edible Harvest: (c) $\mathrm{kg} / \mathrm{yr}$

Dilution Factor: 0.01

(a) Population exposed to contaminated drinking water derived from information obtained from J. Hennigan, Division of Radiation Health, Bureau of Environment and Health, Michigan Department of Public Health.

(b) Drinking water dilution factor estimated by averaging dilution factor derived from $F$ igure 6B-5,

Vol. I of WASH-1258 (1973) suitably weighted for population.

(c) Generic consumption rate used (Table A-1). 


\section{POPULATION DOSE-COMMITMENT ESTIMATES AND \\ AVERAGE INDIVIDUAL DOSE-COMMITMENT HISTOGRAM FOR \\ BIG ROCK POINT}

Dose Commitments (person-rem) from Waterborne Pathways

Total Body

GI-LLI Thyroid

Bone

Liver

Infant

$$
9.8 \mathrm{E}-07
$$

$1.5 \mathrm{E}-07$

$1.7 \mathrm{E}-07$

4.2E-06

$1.2 \mathrm{E}-06$

Child

Teen

Adult

$3.4 \mathrm{E}-02$

$3.4 \mathrm{E}-03$

$7.5 \mathrm{E}-05$

2. $0 \mathrm{E}-01$

$1.9 \mathrm{E}-01$

$6.1 \mathrm{E}-02$

7. $3 \mathrm{E}-03$

5. $4 \mathrm{E}-05$

1. $2 \mathrm{E}-01$

1. $6 \mathrm{E}-01$

6. $5 \mathrm{E}-01$

$6.3 E-02$

$3.5 \mathrm{E}-04$

7. 3E-01

9. $4 E-01$

TOTAL

7. $5 E-01$

7. $4 \mathrm{E}-02$

4.8E- 04

1. $1 \mathrm{E}+00$

1. $3 E+00$

Dose Commitments (person-rem) from Airborne Pathways

Total Body GI-LLI Thyroid Bone Liver Lung

$\begin{array}{lllllll}\text { Infant } & 1.4 \mathrm{E}-03 & 1.4 \mathrm{E}-03 & 1.6 \mathrm{E}-03 & 1.4 \mathrm{E}-03 & 1.4 \mathrm{E}-03 & 1.5 \mathrm{E}-03 \\ \text { Child } & 1.6 \mathrm{E}-02 & 1.6 \mathrm{E}-02 & 1.8 \mathrm{E}-02 & 1.6 \mathrm{E}-02 & 1.6 \mathrm{E}-02 & 1.6 \mathrm{E}-02 \\ \text { Teen } & 1.2 \mathrm{E}-02 & 1.2 \mathrm{E}-02 & 1.2 \mathrm{E}-02 & 1.2 \mathrm{E}-02 & 1.2 \mathrm{E}-02 & 1.2 \mathrm{E}-02 \\ \text { Adult } & 7.1 \mathrm{E}-02 & 7.1 \mathrm{E}-02 & 7.4 \mathrm{E}-02 & 7.0 \mathrm{E}-02 & 7.1 \mathrm{E}-02 & 7.3 \mathrm{E}-02 \\ \text { TOTAL } & 1.0 \mathrm{E}-01 & 1.0 \mathrm{E}-01 & 1.1 \mathrm{E}-01 & 9.9 \mathrm{E}-02 & 1.0 \mathrm{E}-01 & 1.0 \mathrm{E}-01\end{array}$

Production/Consumption factors:
Produce: 1.1
Milk: 6.5
Meat: 1.7
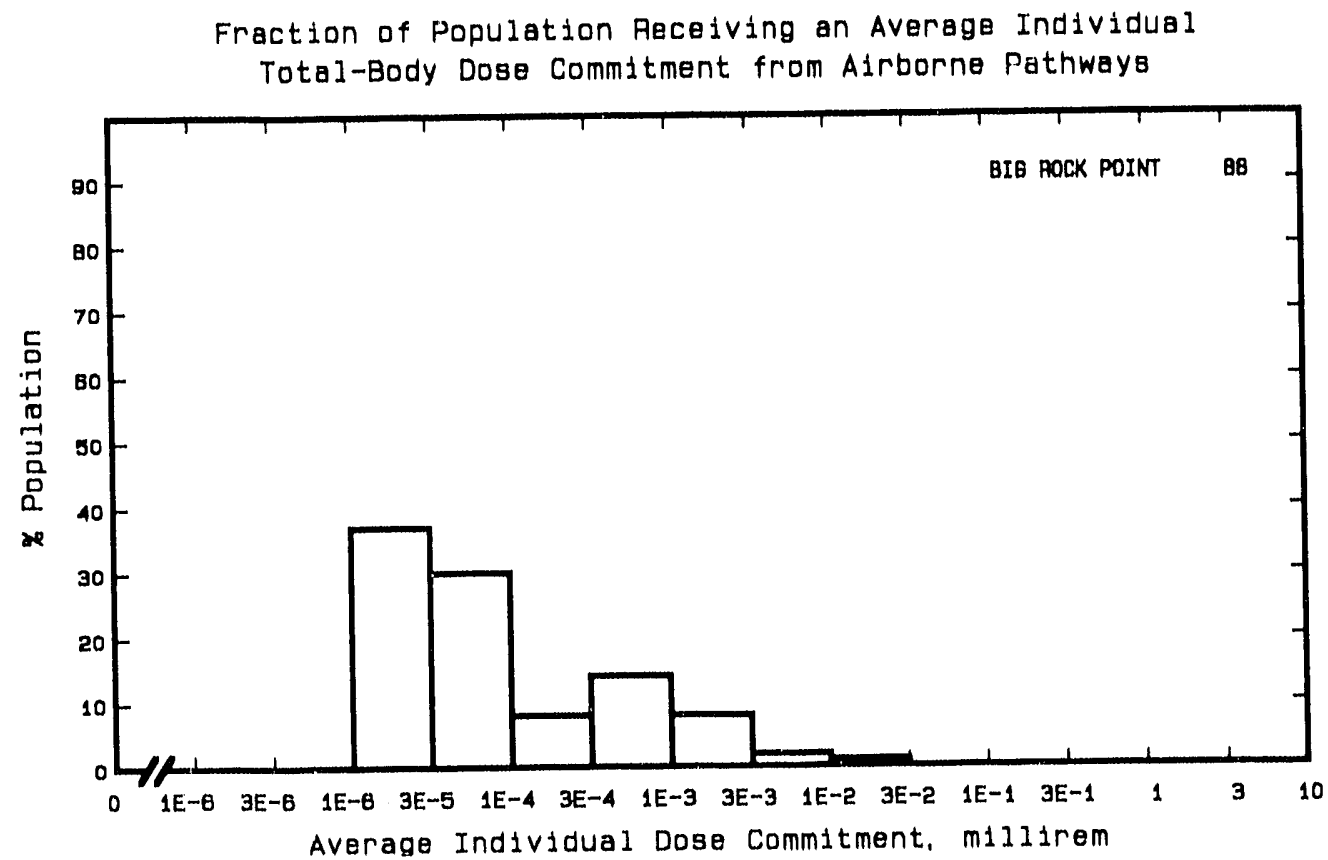
Site: BRAIDWOOD

BRAIDWOOD, ILLINOIS

Location: $\quad N 41.2683^{\circ} \quad W 88.2133^{\circ}$

POPULATION DATA

Total Population Within 2-to-80-km Region: 4.3E6

Major Metropolitan Centers Within Region:

Center

Chicago SMSA

Gary-Hammond-E.Chicago-SMSA

Kankakee SMSA

Aurora

Joliet
Population

$7,100,000(a)$

$640,000(a)$

100,000

81,000

78,000

\section{Location}

$70 \mathrm{~km} \quad \mathrm{NE}$

$70 \mathrm{~km} \quad \mathrm{ENE}$

$32 \mathrm{~km} \quad \mathrm{ESE}$

$55 \mathrm{~km} \quad \mathrm{~N}$

$40 \mathrm{~km} \quad \mathrm{NNE}$

\section{SITE-SPECIFIC DATA - AIRBORNE PATHWAYS}

Average Annual State Production

of Crops and Animal Products

In $80-\mathrm{km}$ Radius Circle

Regional Productivity Factor:

Animal Grazing Factor:

Meteorology Period of Record: 1 JAN 79 - 31 DEC 82 Recovery: $98 \%$
Veg: 1.1E8 kilogram

Milk: 1.8E8 liter

Meat: $1.9 \mathrm{E} 8$ kilogram

0.9

0.5

SITE-SPECIFIC DATA - WATERBORNE PATHWAYS via KANKAKEE RIVER

Average River Flow

at Site: $3,600 \mathrm{ft}^{3} / \mathrm{s}$

Drinking Water: None

Fish:

Edible Harvest: $0.18^{(\mathrm{b})} \mathrm{kg} / \mathrm{yr}$

Dilution Factor: 1

(a) Population of total SMSA given; population of SMSA fraction within $80 \mathrm{~km}$ of site would be somewhat smalier.

(b) Ten percent of population are assumied to obtain fish from river. Average individual consumption rate of $5 \mathrm{~g} / \mathrm{d}$ given in FES (1974) used in lieu of catch data. 


\section{POPULATION DOSE-COMMITMENT ESTIMATES AND \\ AVERAGE INDIVIDUAL DOSE-COMMITMENT HISTOGRAM FOR}

BRAIDWOOD 1 and 2

Dose Commitments (person-rem) from Waterborne Pathways

Total Body GI-LLI Thyroid Bone Liver

$\begin{array}{llllll}\text { Infant } & 0.0 E+00 & 0.0 E+00 & 0.0 E+00 & 0.0 E+00 & 0.0 E+00 \\ \text { Child } & 8.3 E-02 & 1.2 E-01 & 2.6 E-02 & 1.9 E-01 & 2.4 E-01 \\ \text { Teen } & 9.9 E-02 & 2.7 E-01 & 1.9 E-02 & 1.2 E-01 & 2.1 E-01 \\ \text { Adult } & 9.1 E-01 & 2.4 E+00 & 1.3 E-01 & 6.7 E-01 & 1.2 E+00 \\ \text { TOTAL } & 1.1 E+00 & 2.8 E+00 & 1.7 E-01 & 9.8 E-01 & 1.7 E+00\end{array}$

Dose Commitments (person-rem) from Airborne Pathways

Total Body GI-LLI Ihyroid Bone Liver Lung

$\begin{array}{lllllll}\text { Infant } & 7.3 \mathrm{E}-05 & 7.3 \mathrm{E}-05 & 3.7 \mathrm{E}-04 & 4.5 \mathrm{E}-05 & 7.4 \mathrm{E}-05 & 7.6 \mathrm{E}-05 \\ \text { Child } & 1.0 \mathrm{E}-03 & 1.0 \mathrm{E}-03 & 3.3 \mathrm{E}-03 & 4.9 \mathrm{E}-04 & 1.0 \mathrm{E}-03 & 1.1 \mathrm{E}-03 \\ \text { Teen } & 7.2 \mathrm{E}-04 & 7.2 \mathrm{E}-04 & 1.6 \mathrm{E}-03 & 3.6 \mathrm{E}-04 & 7.2 \mathrm{E}-04 & 7.9 \mathrm{E}-04 \\ \text { Adult } & 4.3 \mathrm{E}-03 & 4.3 \mathrm{E}-03 & 7.7 \mathrm{E}-03 & 2.2 \mathrm{E}-03 & 4.3 \mathrm{E}-03 & 4.5 \mathrm{E}-03 \\ \text { TOTAL } & 6.1 \mathrm{E}-03 & 6.1 \mathrm{E}-03 & 1.3 \mathrm{E}-02 & 3.1 \mathrm{E}-03 & 6.1 \mathrm{E}-03 & 6.4 \mathrm{E}-03\end{array}$

Production/Consumption factors:

Produce: 0.12 Milk: 0.28 Meat: 0.49

Fraction of Population Receiving an Average Individual

Total-Body Dose Commitment from Alrborne Pathways

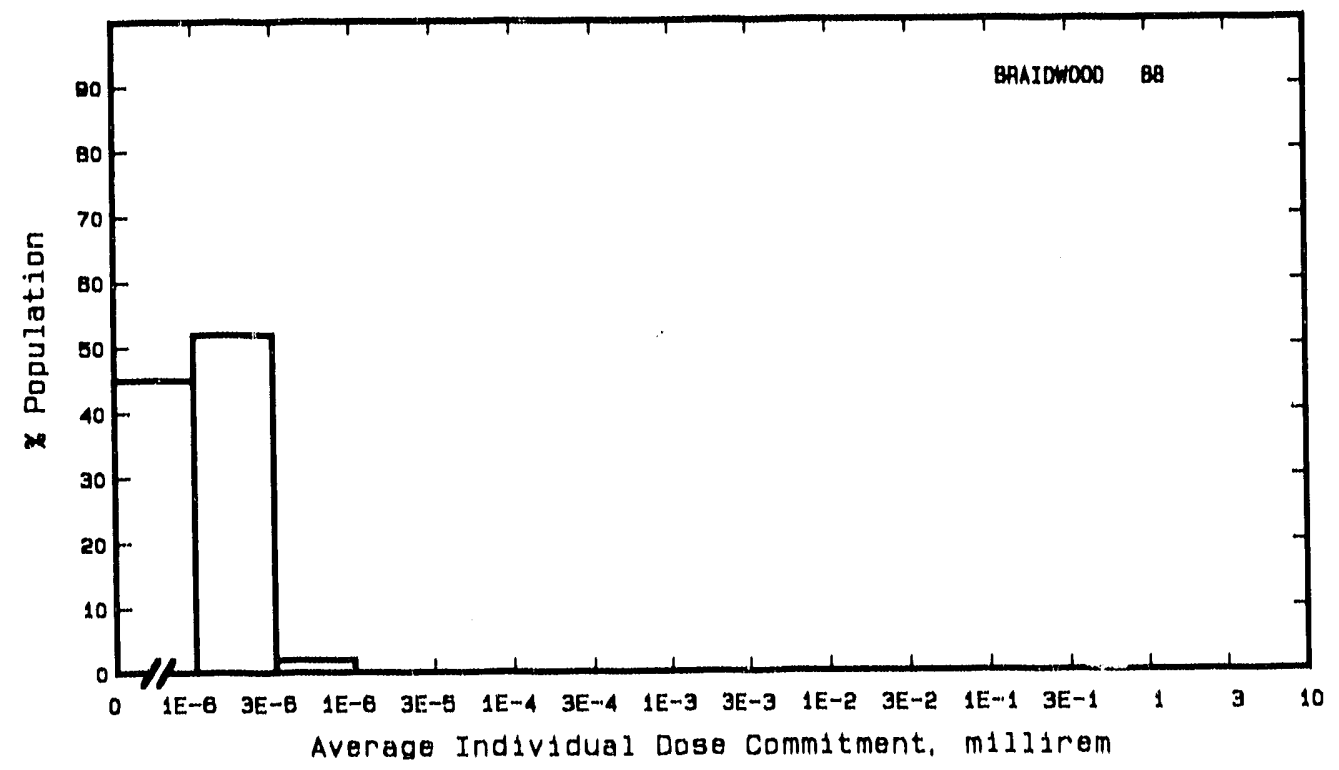


Site: BROWNS FERRY

DECATUR, ALABAMA

Location: $\quad$ N $34.7042^{\circ}$ W $87.1186^{\circ}$

POPULATION DATA

Total Population Within 2-to-80-km Region: 7.5E5

Major Metropolitan Centers Within Region:

Center

Huntsville SMSA

Florence SMSA

Decatur

Athens

Cullman
Population

310,000

140,000

43,000

15,000

13,000
Location

$49 \mathrm{~km} \quad \mathrm{E}$

$52 \mathrm{~km}$ WNW

$16 \mathrm{~km} \quad \mathrm{SE}$

$17 \mathrm{~km}$. NE

$64 \mathrm{~km}$ SSE

SITE-SPECIFIC DATA - AIRBORNE PATHWAYS

Average Annual State Production

Of Crops and Arimal Products

In $80-\mathrm{km}$ Radius Circle

Regional Productivity Factor:

Animal Grazing Factor:
Veg: $1.7 \mathrm{E} 7 \mathrm{k}+1$ logram

Milk: 5.7E7 liter

Meat: $8.6 \mathrm{E} 7 \mathrm{k}+$ logram

1

0.7

Meteorology Period of Record: 1 JAN 74 - 31 DEC 75 Recovery: $94 \%$

SITE-SPECIFIC DATA - WATERBORNE PATHWAYS via TENNESSEE RIVER at WHEELER

Average River Flow

at Site: $45,000 \mathrm{ft}^{3} / \mathrm{s}$

Drinking Water:

Exposed Population: 26,000

Dilution Factor: 1

Fish:

Edible Harvest: $1.6 \mathrm{E} 6 \mathrm{~kg} / \mathrm{yr}$

Dilution Factor: 1 


\section{POPULATION DOSE-COMMITMENT ESTIMATES AND \\ AVERAGE INDIVIDUAL DOSE-COMMITMENT HISTOGRAM FOR \\ BROWNS FERRY 1,2 , AND 3}

Dose Commltments (person-rem) from Waterborne Pathways

Iota1 Body GI-LLI Ihyrotd Bone Liver

Infant

Child

Teen

Adult

$1.8 \mathrm{E}-05$

$3.8 \mathrm{E}-06$

$7.2 E-07$

$1.5 \mathrm{E}-04$

$1.9 E-04$

$4.9 E-02$

2. $2 \mathrm{E}-03$

8.7E-06

2. $8 \mathrm{E}-01$

$3.0 \mathrm{E}-01$

9. $3 E-02$

4. $3 E-03$

3.7E-06

1. $0 E+00$

3. $5 E-02$

3.1E- 05

$1.7 \mathrm{E}-01$

2. 5E-01

TOTAL

1. $2 \mathrm{E}+00$

4.2E-02

4. 4E- 05

9.6E-01

$1.5 \mathrm{E}+00$

2. $O E+00$

Dose Commitments (person-rem) from Atrborne Pathways

Total Body GI-LLI Thyrotd Bone Liver Lung

$\begin{array}{llllllll}\text { Infant } & 1.5 \mathrm{E}-05 & 1.4 \mathrm{E}-05 & 1.4 \mathrm{E}-05 & 1.9 \mathrm{E}-05 & 2.2 \mathrm{E}-05 & 2.9 \mathrm{E}-05 \\ \text { Child } & 1.8 \mathrm{E}-04 & 1.9 \mathrm{E}-04 & 1.6 \mathrm{E}-04 & 2.0 \mathrm{E}-04 & 2.3 \mathrm{E}-04 & 4.1 \mathrm{E}-04 \\ \text { Teen } & 1.3 \mathrm{E}-04 & 1.5 \mathrm{E}-04 & 1.2 \mathrm{E}-04 & 1.2 \mathrm{E}-04 & 1.5 \mathrm{E}-04 & 3.4 \mathrm{E}-04 \\ \text { Adult } & 8.0 \mathrm{E}-04 & 9.8 \mathrm{E}-04 & 7.0 \mathrm{E}-04 & 6.5 \mathrm{E}-04 & 8.1 \mathrm{E}-04 & 1.6 \mathrm{E}-03 \\ \text { TOTAL } & 1.1 \mathrm{E}-03 & 1.3 \mathrm{E}-03 & 9.9 \mathrm{E}-04 & 9.8 \mathrm{E}-04 & 1.2 \mathrm{E}-03 & 2.4 \mathrm{E}-03\end{array}$

Production/Consumption factors:

Produce: $0.12 \quad$ Mrlk: $0.58 \quad$ Meat: 1.4

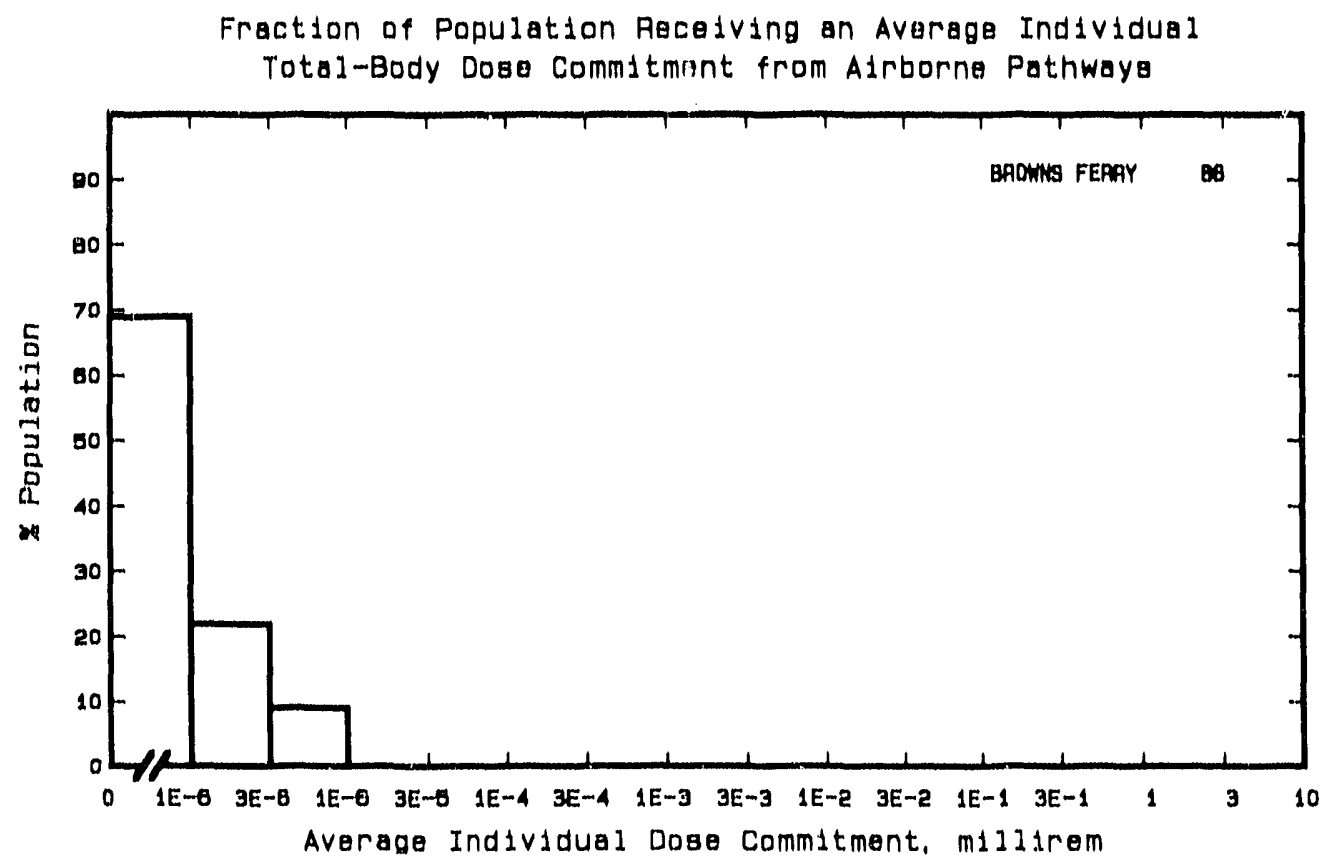


Stte: BRUNSWICK

BRUNSWICK COUNTY, NORTH CAROLINA

Locatton: $\quad N 33.9583^{\circ} \quad W 78.0106^{\circ}$

POPULATION DATA

Total Population Within 2-to-80-km Region: 2.4E5

- Major Metropolitan Centers Within Region:

\begin{tabular}{lccc} 
Center & Population & \multicolumn{2}{l}{ Location } \\
Wilmington SMSA & 140,000 & $32 \mathrm{~km}$ & NNE \\
Whiteville & 5,800 & $75 \mathrm{~km}$ & WNW \\
N. Myrtle Beach & 4,200 & $65 \mathrm{~km}$ & WSW
\end{tabular}

SITE-SPECIFIC DATA - AIRBORNE PATHWAYS

Average Annual State Production

of Crops and Antmal Products

In $80-\mathrm{km}$ Radius Circle

Veg: $2.6 \mathrm{E} 7 \mathrm{k}+$ logram

Milk: 1.0E8 1tter

Meat: $5.8 \mathrm{E} 7 \mathrm{k}+1$ ogram

Regional Productivity Factor:

Animal Grazing Factor:

0.3

0.7

Meteorology Perlod of Record: 1 JAN 75 - 31 DEC 75 Recovery: $93 \%$

SITE-SPECIFIC DATA - WATERBORNE PATHWAYS via ATLANTIC OCEAN

Average Dilution Flow

from Plant: $200 \mathrm{ft}^{3} / \mathrm{s}$

Fish:

Edible Harvest: $2.1 \mathrm{E} 5(\mathrm{a}) \mathrm{kg} / \mathrm{yr}$

Dilution Factor: 0.001

Invertebrates:

Edible Harvest: $1.1 \mathrm{E5}$ (a) $\mathrm{kg} / \mathrm{yr}$

Ditution Factor: 0.002

(a) Fish and invertebrate harvests together total harvest given in FES (1973). 
POPULATION DOSE-COMMITMENT ESTIMATES AND

AVERAGE INDIVIDUAL DOSE-COMMITMENT HISTOGRAM FOR

BRUNSWICK 1 AND 2

Dose Commltments (person-rem) from Waterborne Pathways

\begin{tabular}{|c|c|c|c|c|c|c|}
\hline \multirow[b]{2}{*}{$\begin{array}{l}\text { Infant } \\
\text { Child } \\
\text { Teen } \\
\text { Adult }\end{array}$} & Iotal Bods & GI-LLI & \multicolumn{2}{|c|}{ Thyrotd } & Bone & Liver \\
\hline & $\begin{array}{l}0.0 \mathrm{E}+00 \\
7.1 \mathrm{E}-04 \\
5.1 \mathrm{E}-04 \\
3.4 \mathrm{E}-03\end{array}$ & $\begin{array}{l}0.0 E+00 \\
1.2 E-03 \\
2.5 E-03 \\
2.2 E-02\end{array}$ & \multicolumn{2}{|c|}{$\begin{array}{l}0.0 E+00 \\
1.8 E-04 \\
1.3 E-04 \\
8.5 E-04\end{array}$} & $\begin{array}{l}0.0 E+00 \\
1.1 E-03 \\
6.5 E-04 \\
3.8 E-03\end{array}$ & $\begin{array}{l}0.0 \mathrm{E}+00 \\
1.2 \mathrm{E}-03 \\
1.0 \mathrm{E}-03 \\
6.1 \mathrm{E}-03\end{array}$ \\
\hline \multirow[t]{3}{*}{ TOTAL } & $4.7 E-03$ & $2.5 E-02$ & \multicolumn{2}{|c|}{$1.2 E-03$} & $5.6 E-03$ & $8.4 E-03$ \\
\hline & \multicolumn{6}{|c|}{ Dose Commitments (person-rem) from Airborne Pathways } \\
\hline & Total Body & $\underline{G I-L L I}$ & Thyroid & Bone & Liver & Lung \\
\hline $\begin{array}{l}\text { Infant } \\
\text { Child } \\
\text { Teen } \\
\text { Adult }\end{array}$ & $\begin{array}{l}5.1 E-04 \\
5.7 E-03 \\
4.1 E-03 \\
2.5 E-02\end{array}$ & $\begin{array}{l}5.1 E-04 \\
5.7 E-03 \\
4.2 E-03 \\
2.6 E-02\end{array}$ & $\begin{array}{l}4.1 \mathrm{E}-03 \\
2.7 \mathrm{E}-02 \\
1.3 \mathrm{E}-02 \\
5.5 \mathrm{E}-02\end{array}$ & $\begin{array}{l}5.2 \mathrm{E}-04 \\
5.8 \mathrm{E}-03 \\
4.1 \mathrm{E}-03 \\
2.5 \mathrm{E}-02\end{array}$ & $\begin{array}{l}5.3 \mathrm{E}-04 \\
5.8 \mathrm{E}-03 \\
4.2 \mathrm{E}-03 \\
2.5 \mathrm{E}-02\end{array}$ & $\begin{array}{l}5.5 E-04 \\
6.4 E-03 \\
4.8 E-03 \\
2.8 E-02\end{array}$ \\
\hline TOTAL & $3.5 \mathrm{E}-02$ & $3.6 \mathrm{E}-02$ & $9.9 E-02$ & $3.5 \mathrm{E}-02$ & $3.6 \mathrm{E}-02$ & $3.9 \mathrm{E}-02$ \\
\hline
\end{tabular}

Production/Consumption factors:

Produce: $0.17 \quad$ Milk: $1.0 \quad$ Meat: 0.91

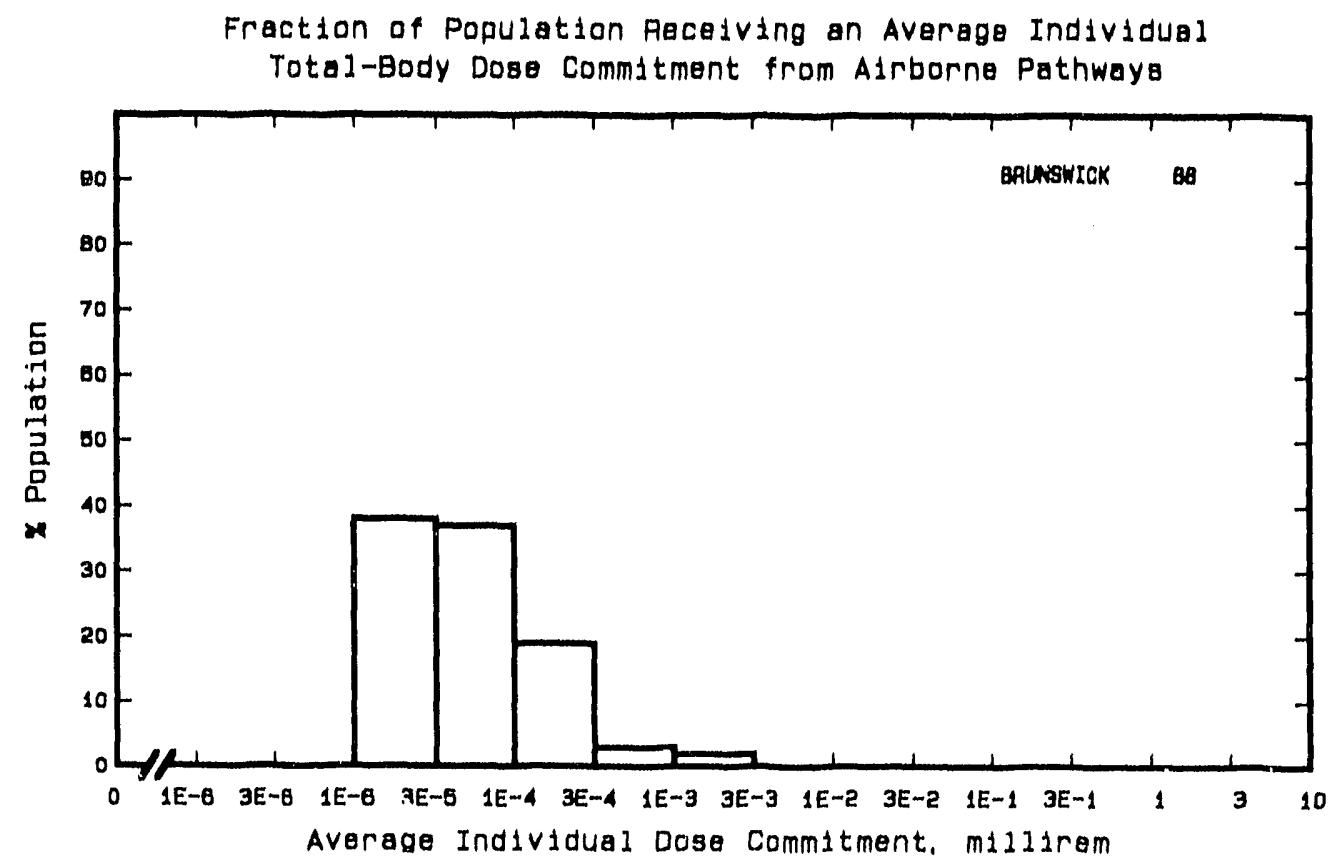


Stte: BYRON

BYRON, ILLINOIS

Location: $\quad N 42.1300^{\circ} \quad W 89.2550^{\circ}$

POPULATION DATA

Total Population Within 2-to-80-km Region: 9.4E5

Major Metropolitan Centers Within Region:

Center

Rockford SMSA

Freeport

Belvedere

Janesville

Beloit

De Kalb

Elgin
Population

280,000

26,000

42,000

61,000

35,000

33,000

64,000

\section{Location}

$27 \mathrm{~km} \quad \mathrm{NE}$

$35 \mathrm{~km} \quad \mathrm{NNW}$

$40 \mathrm{~km} \quad \mathrm{NE}$

$67 \mathrm{~km} \quad \mathrm{~N}$

$48 \mathrm{~km} \quad \mathrm{NNE}$

$48 \mathrm{~km} \quad E S E$

$80 \mathrm{~km} \quad \mathrm{E}$

SITE-SPECIFIC DATA - AIRBORNE PATHWAYS

Average Annual State Production

of Crops and Animal Products

In $80-\mathrm{km}$ Radius Circle

Veg: $1.1 \mathrm{E} 8 \mathrm{k \dagger logram}$

Milk: $1.8 \mathrm{E} 8$ liter

Regional Productivity Factor:

Animal Grazing Factor:

Meat: $1.9 \mathrm{E} 8$ kilogram

Meteorology Period of Record: I JAN 74 - 31 DEC 74 Recovery: $95 \%$

SITE-SPECIFIC DATA - WATERBORNE PATHWAYS via ROCK RIVER

Average River Flow

at Site: $4,600 \mathrm{ft}^{3} / \mathrm{s}$

Drinking Water: None

Fish:

Edible Harvest: $2.1 \mathrm{E} 4 \mathrm{~kg} / \mathrm{yr}$

Dilution Factor: 1 


\section{POPULATION DOSE-COMMITMENT ESTIMATES AND \\ AVERAGE INDIVIDUAL DOSE-COMMITMENT HISTOGRAM FOR \\ BYRON 1 AND 2}

Dose Commitments (person-rem) from Waterborne Pathways

\begin{tabular}{|c|c|c|c|c|c|}
\hline & Total Body & GI-LLI & Thyroid & Bone & Liver \\
\hline $\begin{array}{l}\text { Infant } \\
\text { Child } \\
\text { Teen } \\
\text { Adult }\end{array}$ & $\begin{array}{l}0.0 E+00 \\
4.0 E-03 \\
7.5 E-03 \\
8.0 E-02\end{array}$ & $\begin{array}{l}0.0 \mathrm{E}+00 \\
5.6 \mathrm{E}-03 \\
1.2 \mathrm{E}-02 \\
1.0 \mathrm{E}-01\end{array}$ & $\begin{array}{l}0.0 E+00 \\
4.5 E-04 \\
3.3 E-04 \\
2.3 E-03\end{array}$ & $\begin{array}{l}0.0 E+00 \\
1.7 E-02 \\
1.0 E-02 \\
6.1 E-02\end{array}$ & $\begin{array}{l}0.0 \mathrm{E}+00 \\
2.1 \mathrm{E}-02 \\
1.8 \mathrm{E}-02 \\
1.1 \mathrm{E}-01\end{array}$ \\
\hline TOTAL & $9.2 E-02$ & $1.2 E-01$ & $3.0 E-03$ & $8.9 E-02$ & $1.5 \mathrm{E}-01$ \\
\hline
\end{tabular}

Dose Commitments (person-rem) from Airborne Pathways

Total Body GI-LLI Thyroid Bone Liver Lung

$\begin{array}{lllllll}\text { Infant } & 4.2 \mathrm{E}-04 & 4.1 \mathrm{E}-04 & 6.2 \mathrm{E}-03 & 4.2 \mathrm{E}-04 & 4.3 \mathrm{E}-04 & 4.4 \mathrm{E}-04 \\ \text { Child } & 4.6 \mathrm{E}-03 & 4.6 \mathrm{E}-03 & 5.0 \mathrm{E}-02 & 4.6 \mathrm{E}-03 & 4.7 \mathrm{E}-03 & 5.1 \mathrm{E}-03 \\ \text { Teen } & 3.4 \mathrm{E}-03 & 3.3 \mathrm{E}-03 & 2.1 \mathrm{E}-02 & 3.3 \mathrm{E}-03 & 3.4 \mathrm{E}-03 & 4.0 \mathrm{E}-03 \\ \text { Adult } & 2.0 \mathrm{E}-02 & 2.0 \mathrm{E}-02 & 8.5 \mathrm{E}-02 & 2.0 \mathrm{E}-02 & 2.0 \mathrm{E}-02 & 2.2 \mathrm{E}-02 \\ \text { TOTAL } & 2.9 \mathrm{E}-02 & 2.9 \mathrm{E}-02 & 1.6 \mathrm{E}-01 & 2.8 \mathrm{E}-02 & 2.9 \mathrm{E}-02 & 3.2 \mathrm{E}-02\end{array}$

Production/Consumption factors:

Produce: 0.55 Milk: 1.3 Meat: 2.2

Fraction of Population Peceiving an Average Iridividual Total-Body Dose Commitment from Airborne Pathways

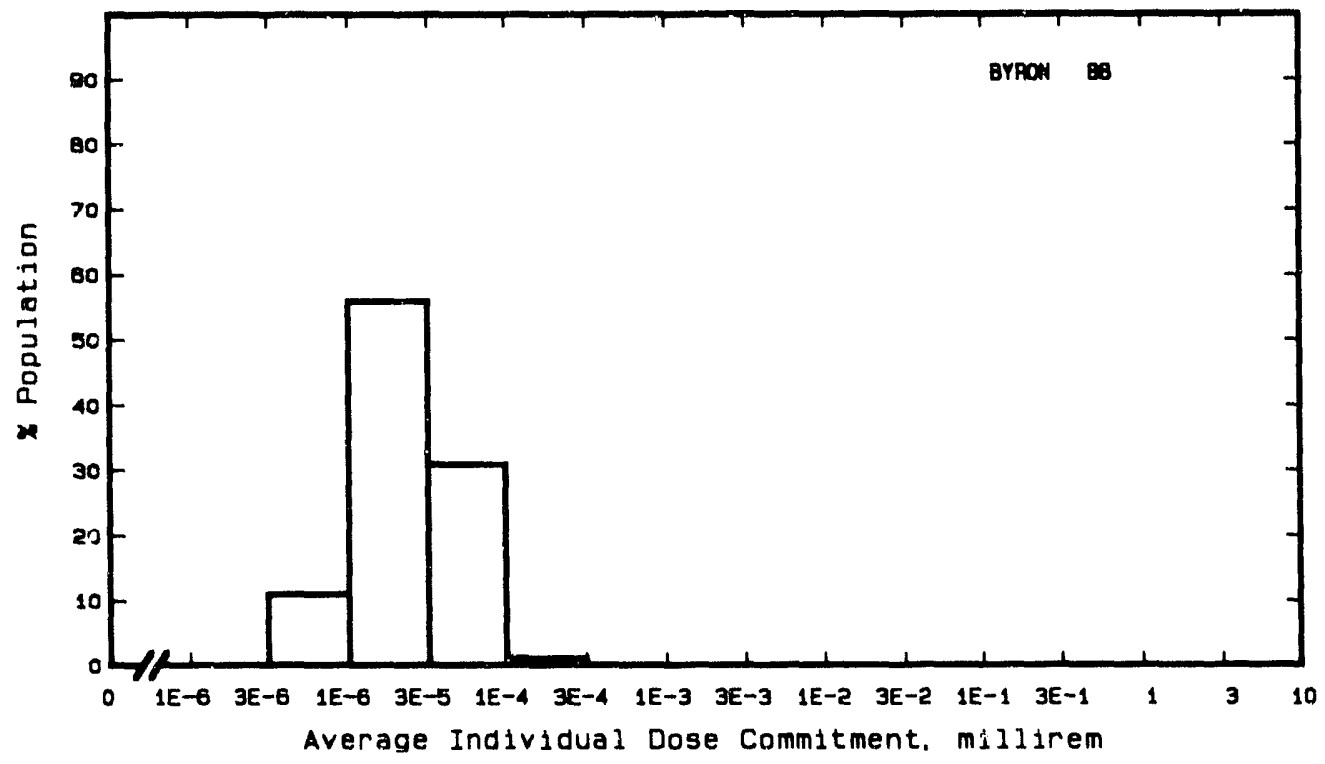


Site: CALLAWAY

FULTON, MISSOURI

Location: $\quad$ N $38.7618^{\circ}$ W $91.7979^{\circ}$

POPULATION DATA

Total Population Within 2-to-80-km Region: 3.7 E5

Major Metropolitan Centers Within Region:

\section{Center}

Columbia

Jefferson City

Mexico

Washington

Fulton
Population

60,000

33,000

12,000

8,500

13,000

\section{Location}

$48 \mathrm{~km} \quad W N W$

$40 \mathrm{~km} \quad W S W$

$45 \mathrm{~km} \quad \mathrm{NNW}$

$69 \mathrm{~km} \quad \mathrm{ESE}$

$19 \mathrm{~km} \mathrm{NW}$

\section{SITE-SPECIFIC DATA - AIRBORNE PATHWAYS}

Average Annual State Production

of Crops and Animal Products

In $80-\mathrm{km}$ Radius Circle

Regional Productivity Factor:

Animal Grazing Factor:
Veg: $3.3 E 7$ kilogram

Milk: 1.5E8 liter

Meat: 1.9 E8 ki Togram

0.9

0.6

Meteorology Period of Record: 4 MAY 74 - 3 MAY 75 Recovery: $89 \%$

Average River Flow

at Site: $80,000 \mathrm{ft}^{3} / \mathrm{s}$

Drinking Water:

None

Fish:

Edible Harvest: $1.0 \mathrm{E} 3 \mathrm{~kg} / \mathrm{yr}$

Dilution Factor: 1 
POPULATION DOSE-COMMITMENT ESTIMATES AND

AVERAGE INDIVIDUAL DOSE-COMMITMENT HISTOGRAM FOR

CALLAWAY

Dose Commitments (person-rem) from Waterborne Pathways

Total Body

GI-LLI

Thyroid

Bone

Liver

Infant

$0.0 E+00$

$0.0 E+00$

$0.0 \mathrm{E}+00$

$0.0 E+00$

$0.0 \mathrm{E}+00$

Child

$3.6 \mathrm{E}-06$

4. $9 \mathrm{E}-07$

1. $5 \mathrm{E}-07$

1.7E-05

1. $9 \mathrm{E}-05$

Teen

$6.7 \mathrm{E}-06$

8. $4 \mathrm{E}-07$

1. $3 \mathrm{E}-07$

1. $0 \mathrm{E}-05$

$1.6 \mathrm{E}-05$

Adult

7.2E-05

7. $0 \mathrm{E}-06$

1. $0 \mathrm{E}-06$

6. $1 \mathrm{E}-05$

9. $6 \mathrm{E}-05$

TOTAL

8.2E-05

8. $4 E-06$

1. $3 \mathrm{E}-06$

8. $9 \mathrm{E}-05$

1. $3 E-04$

Dose Commitments (person-rem) from Airborne Pathways

Total Body GI-LLI Thyroid Bone Liver Lung

$\begin{array}{lllllll}\text { Infant } & 8.9 \mathrm{E}-05 & 8.9 \mathrm{E}-05 & 9.3 \mathrm{E}-05 & 6.5 \mathrm{E}-05 & 8.9 \mathrm{E}-05 & 9.5 \mathrm{E}-05 \\ \text { Child } & 1.1 \mathrm{E}-03 & 1.1 \mathrm{E}-03 & 1.2 \mathrm{E}-03 & 7.2 \mathrm{E}-04 & 1.1 \mathrm{E}-03 & 1.2 \mathrm{E}-03 \\ \text { Teen } & 7.7 \mathrm{E}-04 & 7.7 \mathrm{E}-04 & 7.9 \mathrm{E}-04 & 5.3 \mathrm{E}-04 & 7.7 \mathrm{E}-04 & 8.9 \mathrm{E}-04 \\ \text { Adult } & 4.5 \mathrm{E}-03 & 4.5 \mathrm{E}-03 & 4.6 \mathrm{E}-03 & 3.2 \mathrm{E}-03 & 4.5 \mathrm{E}-03 & 4.9 \mathrm{E}-03 \\ \text { TOTAL } & 6.5 \mathrm{E}-03 & 6.5 \mathrm{E}-03 & 6.6 \mathrm{E}-03 & 4.5 \mathrm{E}-03 & 6.5 \mathrm{E}-03 & 7.1 \mathrm{E}-03\end{array}$

Production/Consumption factors:

Produce: 0.41

Milk: 2.8

Meat: 5.8

Fraction of Population Receiving an Average Individual Total-Body Dose Commitment from Airborne Pathwaya

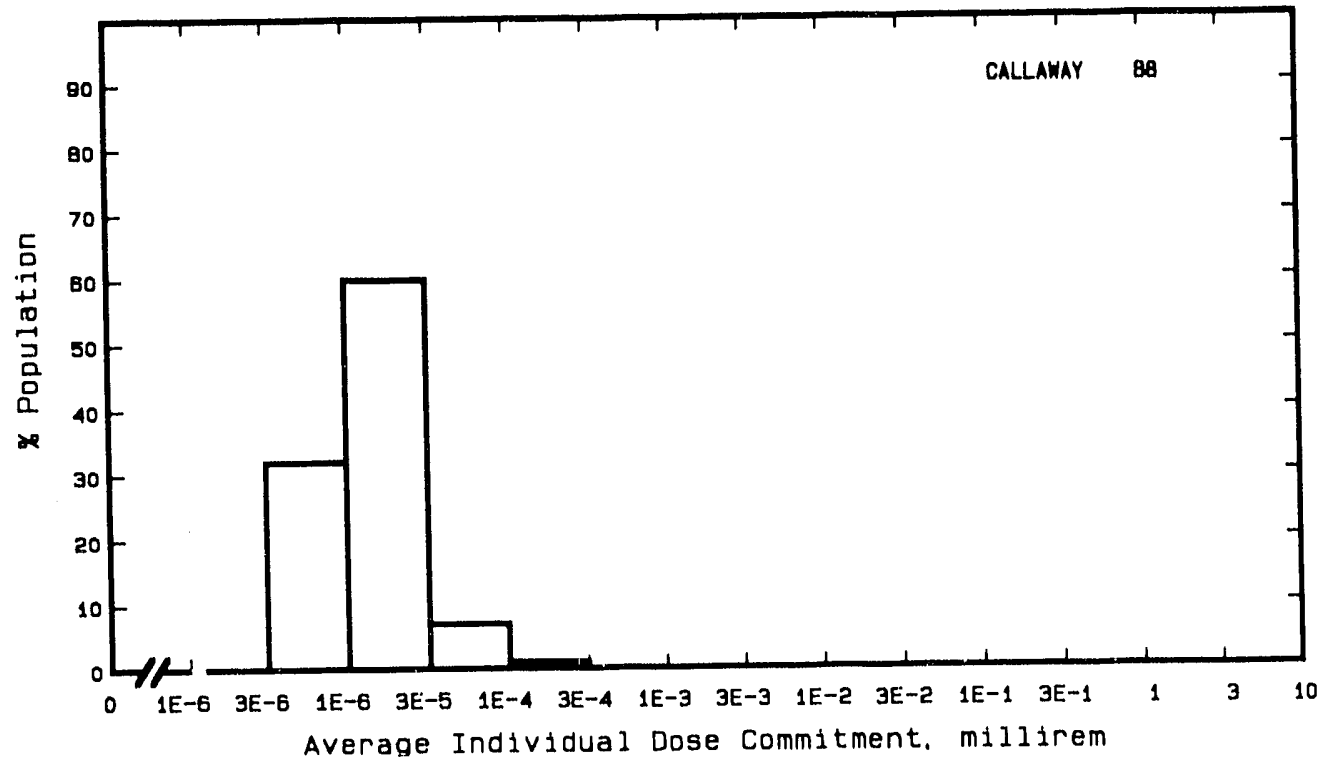


Site: CALVERT CLIFFS

LUSBY, MARYLAND

Location: $\quad N 38.4347^{\circ} \quad W 76.4419^{\circ}$

POPULATION DATA

Total Population Within 2-to-80-km Region: 2.8E6

Major Metropolitan Centers Within Region:

Center

Washington, DC SMSA (3/4)

Bowie

Annapol is

Waldorf

Salisbury

Cambridge
Population

$2,700,000$

35,000

33,000

10,800

16,000

12,000
Location

$73 \mathrm{~km} \mathrm{NW}$

$71 \mathrm{~km} \quad \mathrm{NNW}$

$61 \mathrm{~km} \mathrm{~N}$

$46 \mathrm{~km} \quad W N W$

$75 \mathrm{~km} \quad \mathrm{E}$

$32 \mathrm{~km} \quad \mathrm{ENE}$

SITE-SPECIFIC DATA - AIRBORNE PATHWAYS

Average Annual State Production

of Crops and Animal Products

In $80-\mathrm{km}$ Radius Circle

Veg: 4.5E7 kilogram

Milk: 5.0E8 liter

Regional Productivity Factor:

Meat: $6.2 \mathrm{E} 7$ kilogram

Animal Grazing Factor:

0.6

0.6

Meteorology Period of Record: 1 JAN 75 - 31 DEC 75 Recovery: 96\%

SITE-SPECIFIC DATA - WATERBORNE PATHWAYS via CHESAPEAKE BAY

Average Dilution Flow

from Plant: $2,700 \mathrm{ft}^{3} / \mathrm{s}$

Fish:

Edible Harvest: $1.0 \mathrm{E} 7 \mathrm{~kg} / \mathrm{yr}$

Invertebrates:

Edible Harvest: $7.4 \mathrm{E} 6 \mathrm{~kg} / \mathrm{yr}$
Dilution Factor: $0.062(\mathrm{~g})$

(a) Dilutions given in FES (1973). 
POPULATION DOSE-COMMITMENT ESTIMATES AND

AVERAGE INDIVIDUAL DOSE-COMMITMENT HISTOGRAM FOR

CALVERT CLIFFS 1 AND 2

Dose Commitments (person-rem) from Waterborne Pathways

Total Body GI-LLI Thyroid Bone Liver

\begin{tabular}{|c|c|c|c|c|c|c|}
\hline $\begin{array}{l}\text { Infant } \\
\text { Child } \\
\text { Teen } \\
\text { Adult }\end{array}$ & $\begin{array}{l}0.0 \mathrm{E}+00 \\
7.9 \mathrm{E}-02 \\
1.3 \mathrm{E}-01 \\
1.3 \mathrm{E}+00\end{array}$ & $\begin{array}{l}0.0 \\
4.2 \\
8.8 \\
7.7\end{array}$ & & $\begin{array}{l}0.0 \mathrm{E}+00 \\
3.1 \mathrm{E}-01 \\
2.2 \mathrm{E}-01 \\
1.4 \mathrm{E}+00\end{array}$ & $\begin{array}{l}0.0 E+00 \\
3.0 E-01 \\
1.8 E-01 \\
1.0 E+00\end{array}$ & $\begin{array}{l}0.0 E+00 \\
3.5 E-01 \\
2.9 E-01 \\
1.7 E+00\end{array}$ \\
\hline \multirow[t]{3}{*}{ TOTAL } & $1.5 \mathrm{E}+00$ & 9.0 & & $2.0 E+00$ & $1.5 E+00$ & $2.4 \mathrm{E}+00$ \\
\hline & \multicolumn{6}{|c|}{ Dose Commitments (person-rem) from Airborne Pathways } \\
\hline & Total Body & $\underline{G I-L L I}$ & Thyroid & Bone & Liver & Lung \\
\hline $\begin{array}{l}\text { Infant } \\
\text { Child } \\
\text { Teen } \\
\text { Adult }\end{array}$ & $\begin{array}{l}3.7 \mathrm{E}-03 \\
4.0 \mathrm{E}-02 \\
2.9 \mathrm{E}-02 \\
1.7 \mathrm{E}-01\end{array}$ & $\begin{array}{l}3.3 \mathrm{E}-03 \\
3.7 \mathrm{E}-02 \\
2.7 \mathrm{E}-02 \\
1.6 \mathrm{E}-01\end{array}$ & $\begin{array}{l}1.8 \mathrm{E}-01 \\
9.4 \mathrm{E}-01 \\
3.8 \mathrm{E}-01 \\
1.3 \mathrm{E}+00\end{array}$ & $\begin{array}{l}5.2 E-03 \\
4.9 E-02 \\
2.9 E-02 \\
1.6 E-01\end{array}$ & $\begin{array}{l}6.0 \mathrm{E}-03 \\
5.3 \mathrm{E}-02 \\
3.3 \mathrm{E}-02 \\
1.8 \mathrm{E}-01\end{array}$ & $\begin{array}{l}3.8 E-03 \\
4.2 E-02 \\
3.2 E-02 \\
1.7 E-01\end{array}$ \\
\hline TOTAL & $2.4 E-01$ & $2.3 E-01$ & $2.8 E+00$ & 2. $4 \mathrm{E}-01$ & $2.7 E-01$ & $2.5 E-01$ \\
\hline
\end{tabular}

Production/Consumption factors:

Produce: 0.049 Milk: 0.80 Meat: 0.16

Fraction of Population Receiving an Average Individual

Tota1-Body Dose Commitment from Alrborne Pathwaya

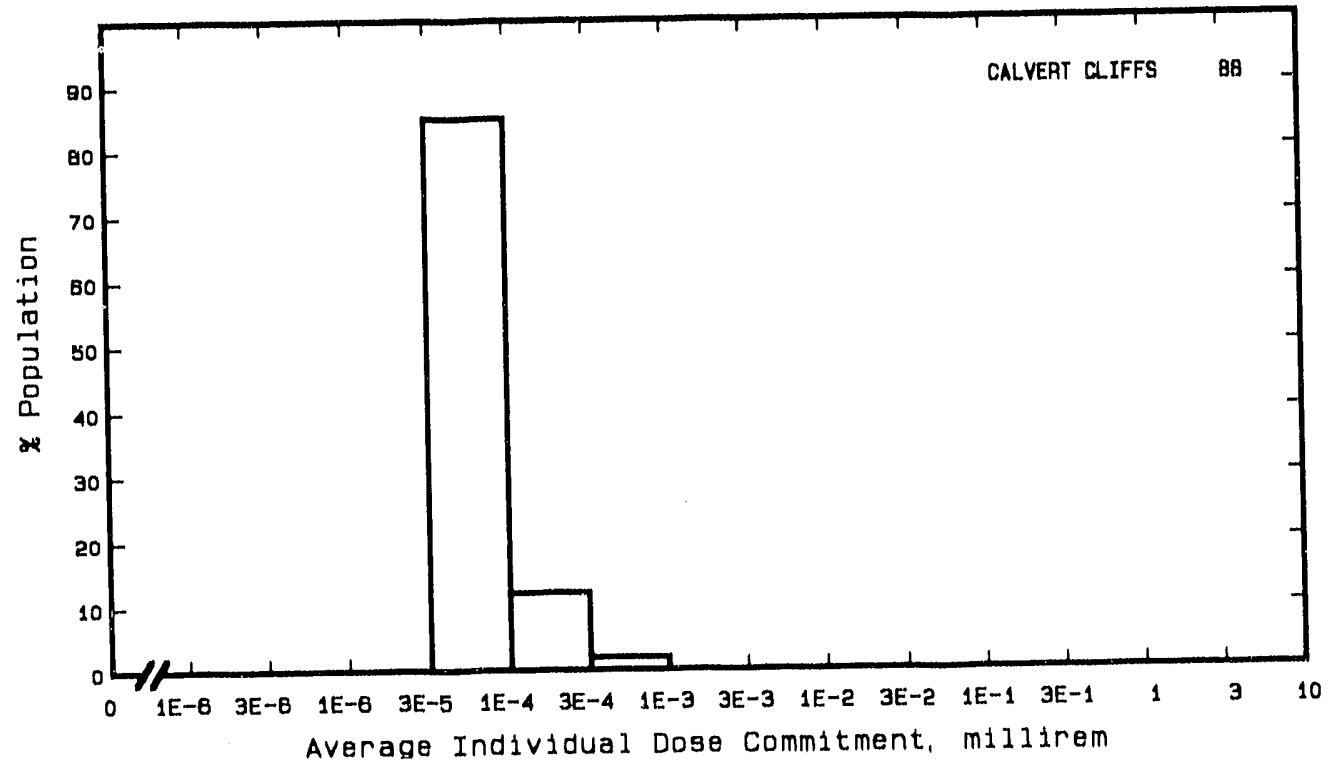


Stte: CATAWBA

CLOVER, SOUTH CAROLINA

Location: $\quad$ N $34.9950^{\circ} \quad$ W $81.2450^{\circ}$

POPULATION DATA

Total Population Within 2-to-80-km Region: 1.6E6

Major Metropolitan Centers Within Region:

Center Population Location

$\begin{array}{lrrl}\text { Charlotte-Gastonia SMSA } & 680,000 & 29 \mathrm{~km} & \mathrm{NE} \\ \text { Kannapolis } & 37,000 & 64 \mathrm{~km} & \mathrm{NE} \\ \text { Rock Hill } & 37,000 & 11 \mathrm{~km} & \mathrm{~S} \\ \text { Spartanburg } & 16,000 & 80 \mathrm{~km} & \text { W }\end{array}$

SITE-SPECIFIC DATA - AIRBORNE PATHWAYS

Average Annual State Production

of Crops and Animal Products

In $80-\mathrm{km}$ Radius Circle

Regional Productivity Factor:

Animal Grazing Factor:

Meteorology Period of Record: 17 DEC 75 - 16 DEC 76 Recovery: 94\%
Veg: $7.4 \mathrm{E} 6 \mathrm{kilogram}$

Milk: $5.7 E 7$ liter

Meat: $5.0 E 7$ kilogram

0.9

0.75

SITE-SPECIFIC DATA - WATERBORNE PATHWAYS via CATAWBA RIVER

Average River Flow

at Site: $4,400 \mathrm{ft}^{3} / \mathrm{s}$

Drinking Water:

None

Fish:

Edible Harvest: $1.0 \mathrm{E} 6 \mathrm{~kg} / \mathrm{yr}$ Dilution Factor: 1 


\section{POPULATION DOSE-COMMITMENT ESTIMATES AND AVERAGE INDIVIDUAL DOSE-COMMITMENT HISTOGRAM FOR}

CATAWBA 1 AND 2

Dose Commitments (person-rem) from Waterborne Pathways

\begin{tabular}{|c|c|c|c|c|}
\hline Total Body & GI-LLI & Thyroid & Bone & Liver \\
\hline $\begin{array}{l}0.0 E+00 \\
6.2 E-02 \\
1.1 E-01 \\
1.2 E+00\end{array}$ & $\begin{array}{l}0.0 E+00 \\
9.6 E-02 \\
2.0 E-01 \\
1.7 E+00\end{array}$ & $\begin{array}{l}0.0 \mathrm{E}+00 \\
1.6 \mathrm{E}-02 \\
1.2 \mathrm{E}-02 \\
8.1 \mathrm{E}-02\end{array}$ & $\begin{array}{l}0.0 E+00 \\
2.8 E-01 \\
1.7 E-01 \\
9.6 E-01\end{array}$ & $\begin{array}{l}0.0 E+00 \\
3.3 E-01 \\
2.8 E-01 \\
1.7 E+00\end{array}$ \\
\hline $1.4 E+00$ & $2.0 E+00$ & $1.1 E-01$ & 1. $4 E+00$ & 2. $3 E+00$ \\
\hline
\end{tabular}

Dose Commitments (person-rem) from Airborne Pathways

Total Body GI-LLI Thyroid Bone Liver Lung

$\begin{array}{lllllll}\text { Infant } & 2.8 \mathrm{E}-03 & 2.8 \mathrm{E}-03 & 3.5 \mathrm{E}-03 & 2.5 \mathrm{E}-03 & 2.8 \mathrm{E}-03 & 3.0 \mathrm{E}-03 \\ \text { Chi1d } & 3.3 \mathrm{E}-02 & 3.3 \mathrm{E}-02 & 3.8 \mathrm{E}-02 & 2.7 \mathrm{E}-02 & 3.3 \mathrm{E}-02 & 3.6 \mathrm{E}-02 \\ \text { Teen } & 2.4 \mathrm{E}-02 & 2.5 \mathrm{E}-02 & 2.7 \mathrm{E}-02 & 2.0 \mathrm{E}-02 & 2.5 \mathrm{E}-02 & 2.9 \mathrm{E}-02 \\ \text { Adult } & 1.5 \mathrm{E}-01 & 1.5 \mathrm{E}-01 & 1.6 \mathrm{E}-01 & 1.2 \mathrm{E}-01 & 1.5 \mathrm{E}-01 & 1.6 \mathrm{E}-01 \\ \text { TOTAL } & 2.1 \mathrm{E}-01 & 2.1 \mathrm{E}-01 & 2.3 \mathrm{E}-01 & 1.7 \mathrm{E}-01 & 2.1 \mathrm{E}-01 & 2.3 \mathrm{E}-01\end{array}$

Production/Consumption factors:

Produce: $0.022 \quad$ Milk: 0.25 Meat: 0.35

Fraction of Population Feceiving an Average Individual Tota1-Body Dose Comm1tment from Airborne Pathways

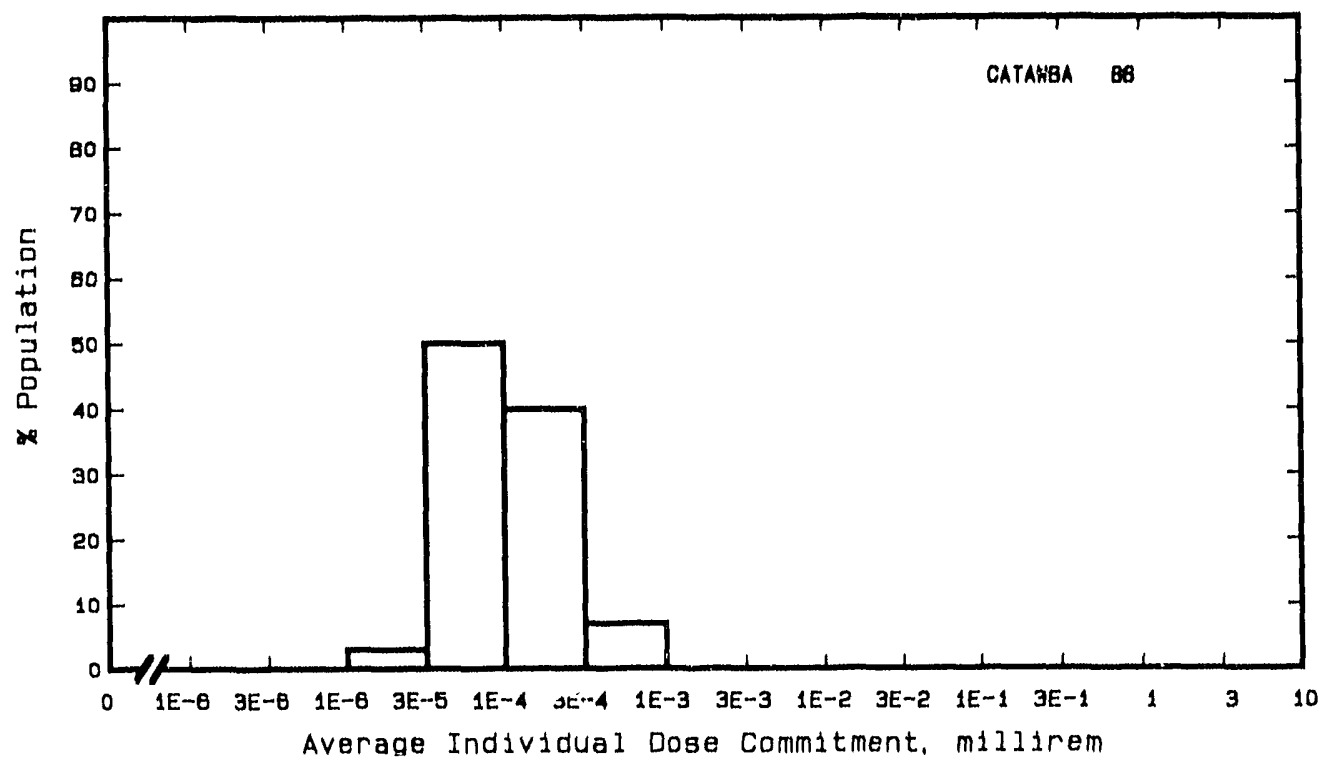


Site: CLINTON

CLINTON, ILLINOIS

Location: $\quad N 40.1517^{\circ} \quad$ W $88.9533^{\circ}$

POPULATION DATA

Total Population Within 2-to-80-km Region: 2.7E6

Major Metropolitan Centers Within Region:

Center

Decatur

Springfield

Champaign-Urbana

Bloomington-Normal
Population

94,000

100,000

94,000

80,000
Location

$32 \mathrm{~km} \mathrm{~S}$

$72 \mathrm{~km} \mathrm{SW}$

$50 \mathrm{~km} \quad \mathrm{E}$

$35 \mathrm{~km} \quad \mathrm{NNW}$

\section{SITE-SPECIFIC DATA - AIRBORNE PATHWAYS}

Average Annual State Production

Of Crops and Animai Products

In $80-\mathrm{km}$ Radius Circle

Regional Productivity Factor:

Animal Grazing Factor:

Meteorology Period of Record: 14 MAR 72 - 28 SEP 79 Recovery: 91\%
Veg: 1.1 E8 kilogram

Milk: $1.8 \mathrm{E} 8$ liter

Meat: $1.9 \mathrm{E} 8$ kilogram

0.9

0.5

SITE-SPECIFIC DATA - WATERBORNE PATHWAYS via CLINTON LAKE

Average Dilution Flow

from Plant: $0.38 \mathrm{ft}^{3} / \mathrm{s}$

Drinking Water: None

Fish:

Edible Harvest: $0.18(\mathrm{a}) \mathrm{kg} / \mathrm{yr}$

Dilution Factor: 0.25

(a) Ten percent of population are assumed to obtain fish from lake. Average individual consumption rate of $5 \mathrm{~g} / \mathrm{d}$ given in FES (1974) used in lieu of catch data. 
POPULATION DOSE-COMMITMENT ESTIMATES AND

AVERAGE INDIVIDUAL DOSE-COMMITMENT HISTOGRAM FOR

\section{CLINTON 1}

Dose Commitments (person-rem) from Waterhorne Pathways

\begin{tabular}{|c|c|c|c|c|c|c|}
\hline & Iotal Bod & GI- & & Thyrotd & Bone & Liver \\
\hline $\begin{array}{l}\text { Infant } \\
\text { Child } \\
\text { Teen } \\
\text { Adult }\end{array}$ & $\begin{array}{l}0.0 E+00 \\
6.4 E-01 \\
4.6 E-01 \\
2.9 E+00\end{array}$ & $\begin{array}{l}0.0 \mathrm{E} \\
1.5 \mathrm{E} \\
3.4 \mathrm{E} \\
3.1 \mathrm{E}\end{array}$ & & $\begin{array}{l}0.0 \mathrm{E}+00 \\
1.3 \mathrm{E}-02 \\
1.1 \mathrm{E}-02 \\
9.0 \mathrm{E}-02\end{array}$ & $\begin{array}{l}0.0 E+00 \\
1.5 E+00 \\
9.6 E-01 \\
6.1 E+00\end{array}$ & $\begin{array}{l}0.0 E+00 \\
1.3 E+00 \\
1.2 E+00 \\
7.5 E+00\end{array}$ \\
\hline TOTAL & $4.0 E+00$ & $3.5 \mathrm{E}$ & & $1.1 \mathrm{E}-01$ & $8.5 \mathrm{E}+00$ & $1.0 E+01$ \\
\hline & Dose Commit & lents (per & on-rem) $f$ & from Atrborn & Pathways & \\
\hline & Iotal Body & GI-LLI & Thyroid & Bone & Liver & Lung \\
\hline $\begin{array}{l}\text { Infant } \\
\text { Child } \\
\text { Teen } \\
\text { Adult }\end{array}$ & $\begin{array}{l}9.7 \mathrm{E}-05 \\
1.6 \mathrm{E}-03 \\
1.0 \mathrm{E}-03 \\
6.0 \mathrm{E}-03\end{array}$ & $\begin{array}{l}9.7 \mathrm{E}-05 \\
1.6 \mathrm{E}-03 \\
1.2 \mathrm{E}-03 \\
7.0 \mathrm{E}-03\end{array}$ & $\begin{array}{l}2.1 \mathrm{E}-04 \\
2.4 \mathrm{E}-03 \\
1.4 \mathrm{E}-03 \\
7.5 \mathrm{E}-03\end{array}$ & $\begin{array}{l}3.9 E-05 \\
4.7 E-04 \\
3.2 E-04 \\
1.9 E-03\end{array}$ & $\begin{array}{l}9.7 \mathrm{E}-05 \\
1.5 \mathrm{E}-03 \\
1.0 \mathrm{E}-03 \\
6.0 \mathrm{E}-03\end{array}$ & $\begin{array}{l}1.5 E-04 \\
2.4 E-03 \\
1.9 E-03 \\
9.5 E-03\end{array}$ \\
\hline TOTAL & $8.7 E-03$ & $9.9 \mathrm{E}-03$ & $1.2 \mathrm{E}-02$ & $2.8 E-03$ & $8.7 E-03$ & $1.4 \mathrm{E}-02$ \\
\hline
\end{tabular}

Production/Consumption factors:
Produce: 0.19
Milk: $\quad 0.45$
Meat: 0.78

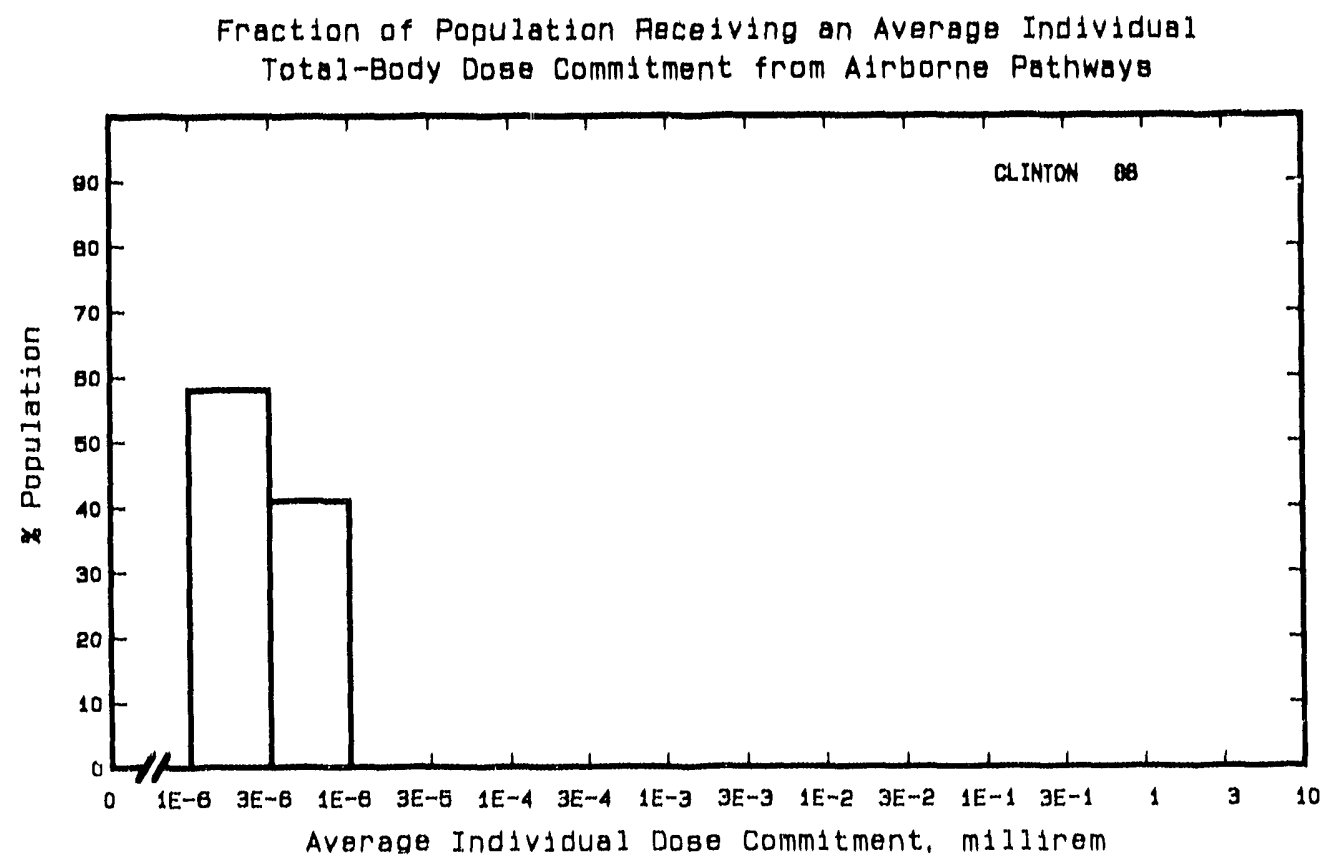


Site: COOK

BENTON HARBOR, MICHIGAN

Location: $\quad$ N $41.9761^{\circ} \quad$ W $86.5664^{\circ}$

POPULATION DATA

Total Population Within 2-to-80-km Region: $1.2 E 6$

Major Metropolttan Centers Within Region:

\begin{tabular}{lcccc}
\multicolumn{1}{c}{ Center } & Population & \multicolumn{2}{c}{ Location } \\
Gary & 140,000 & $77 \mathrm{~km}$ & SW \\
South Bend SMSA & 280,000 & $42 \mathrm{~km}$ & SE \\
Elkhart SMSA & 140,000 & $58 \mathrm{~km}$ & SE \\
Mishiwaka & 40,000 & $47 \mathrm{~km}$ & SE \\
Michigan City & 37,000 & $40 \mathrm{~km}$ & SW
\end{tabular}

SITE-SPECIFIC DATA - AIRBORNE PATHWAYS

Average Annual State Production

of Crops and Animal Products

In $80-\mathrm{km}$ Radius Circle

Regional Productivity Factor:

Animal Grazing Factor:
Veg: $1.1 \mathrm{E} 8 \mathrm{k}+\mathrm{log} r \mathrm{am}$

Ml1k: 2.3E8 1iter

Meat: $1.9 \mathrm{E} 8 \mathrm{kflogram}$

0.6

0.5

Meteorology Period of Record: 1 MAY 75 - 31 APR 76 Recovery: $95 \%$

Average Dilution Flow

from Plant: $2,500 \mathrm{ft}^{3} / \mathrm{s}$

Drinking Water:

Exposed Population: 269,000
Dilution Factor: $0.025(a)$

Fish:

Edible Harvest: $1.5 \mathrm{E} 6 \mathrm{~kg} / \mathrm{yr}$

Dilution Factor: 0.01

(a) Drinking water dllution factor est lmated by averaging dilution factors dertved from FES (1973) suitably weighted for population. 
POPULATION DOSE-COMMITMENT ESTIMATES AND

AVERAGE INDIVIDUAL DOSE-COMMITMENT HISTOGRAM FOR

COOK 1 AND 2

Dose Commitments (person-rem) from Waterborne Pathways

Iotal Body GI-HLI Ihyrotd Bone Liver

$\begin{array}{llllll}\text { Infant } & 2.4 \mathrm{E}-03 & 2.4 \mathrm{E}-03 & 5.9 \mathrm{E}-03 & 2.6 \mathrm{E}-04 & 2.7 \mathrm{E}-03 \\ \text { Cht1d } & 3.1 \mathrm{E}-02 & 2.8 \mathrm{E}-02 & 5.2 \mathrm{E}-02 & 1.8 \mathrm{E}-02 & 4.8 \mathrm{E}-02 \\ \text { Teen } & 1.7 \mathrm{E}-02 & 1.2 \mathrm{E}-02 & 1.8 \mathrm{E}-02 & 1.02-02 & 2.7 \mathrm{E}-02 \\ \text { Adu1t } & 1.6 \mathrm{E}-01 & 1.0 \mathrm{E}-01 & 1.4 \mathrm{E}-01 & 5.9 \mathrm{E}-02 & 1.9 \mathrm{E}-01 \\ \text { TOTAL } & 2.1 \mathrm{E}-01 & 1.4 \mathrm{E}-01 & 2.2 \mathrm{E}-01 & 8.7 \mathrm{E}-02 & 2.7 \mathrm{E}-01\end{array}$

Dose Commitments (person-rem) from Atrborne Pathways

Iotal Body GI-LLI Ihyrotd Bone Liver Lung

$\begin{array}{lllllll}\text { Infant } & 7.8 \mathrm{E}-05 & 6.7 \mathrm{E}-05 & 3.6 \mathrm{E}-03 & 1.3 \mathrm{E}-04 & 1.7 \mathrm{E}-04 & 7.9 \mathrm{E}-05 \\ \text { Child } & 9.7 \mathrm{E}-04 & 8.1 \mathrm{E}-04 & 2.4 \mathrm{E}-02 & 1.4 \mathrm{E}-03 & 1.7 \mathrm{E}-03 & 9.4 \mathrm{E}-04 \\ \text { Teen } & 7.1 \mathrm{E}-04 & 5.7 \mathrm{E}-04 & 9.4 \mathrm{E}-03 & 6.7 \mathrm{E}-04 & 9.3 \mathrm{E}-04 & 6.7 \mathrm{E}-04 \\ \text { Adult } & 4.2 \mathrm{E}-03 & 3.4 \mathrm{E}-03 & 3.3 \mathrm{E}-02 & 3.3 \mathrm{E}-03 & 4.5 \mathrm{E}-03 & 3.7 \mathrm{E}-0.3 \\ \text { TOTAL } & 5.9 \mathrm{E}-03 & 4.8 \mathrm{E}-03 & 7.0 \mathrm{E}-02 & 5.5 \mathrm{E}-03 & 7.2 \mathrm{E}-03 & 5.4 \mathrm{E}-03\end{array}$

Production/Consumption factors:

Produce: 0.29 Milk: 0.91 Meat: 1.2

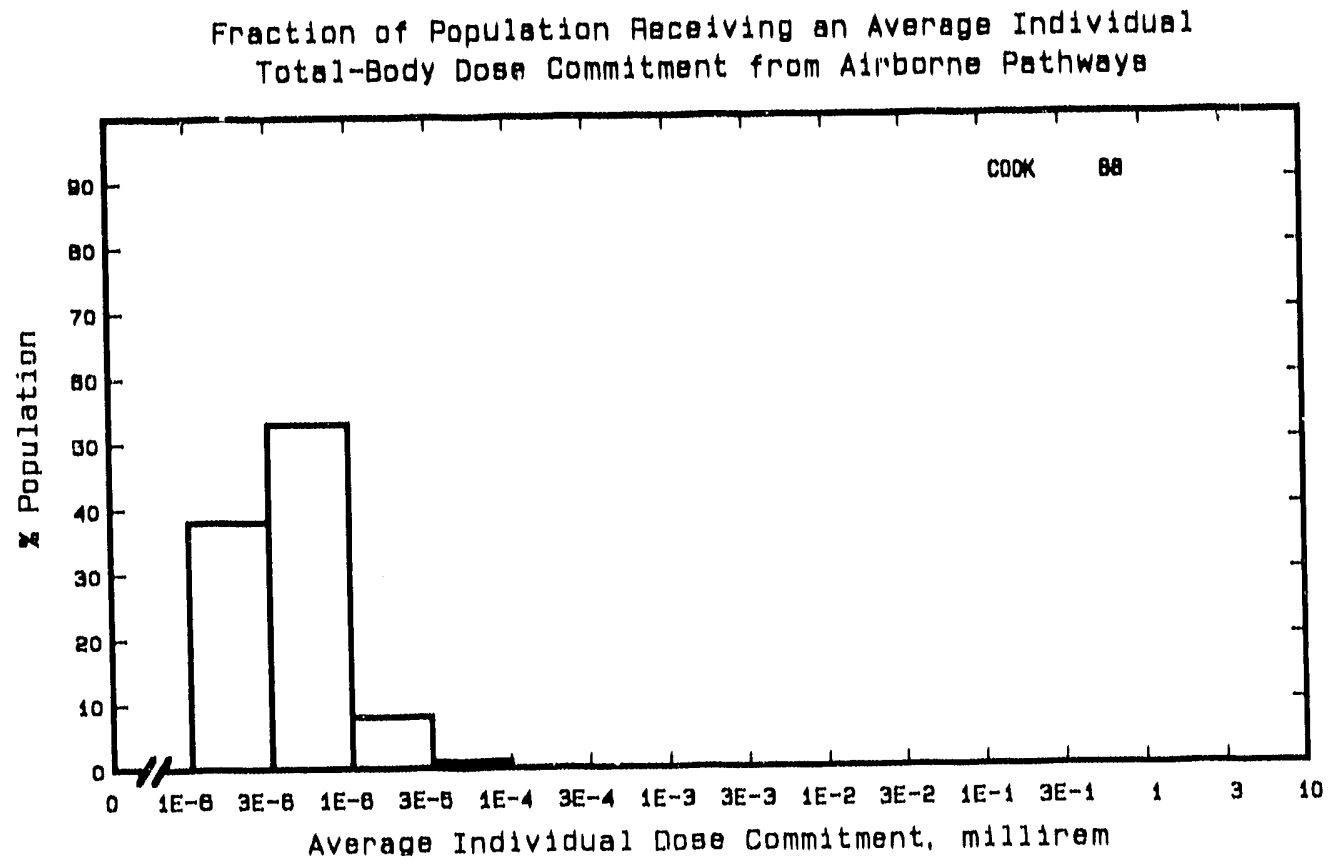


Site: COOPER

NEMAHA COUNTY, NEBRASKA

Location: $\quad$ N $40.3619^{\circ} \quad$ W $95.6411^{\circ}$

POPULATION DATA

Total Population Within 2-to-80-km Region: $1.7 \mathrm{E} 5$

Major Metropolitan Centers Within Region:

\begin{tabular}{lcccc}
\multicolumn{1}{c}{ Center } & Populatton & \multicolumn{3}{c}{ Location } \\
\cline { 2 - 4 } & 7,100 & $40 \mathrm{~km}$ & NNW \\
Nebraska City & 6,800 & $80 \mathrm{~km}$ & NNE \\
Red Oak & 6,300 & $76 \mathrm{~km}$ & NNIW \\
Plattsmouth & 6,300 & $51 \mathrm{~km}$ & NNE \\
Shenandoah & 5,400 & $33 \mathrm{~km}$ & $\mathrm{~S}$
\end{tabular}

SITE-SPECIFIC DATA - AIRBORNE PATHWAYS

Average Annual State Production

of Crops and Antmal Products

In $80-\mathrm{km}$ Radius Circle

Regional Productivity Factor:

Animal Grazing Factor:

Meteorology Pertod of Record: 1 MAR 70 - 31 DEC 75 Recovery: $89 \%$
Veg: $9.7 \mathrm{E} 7 \mathrm{kilogram}$

Milk: $7.2 \mathrm{E} 7$ 1 lter

Meat: 2.0E8 kilogram

1

0.6

SITE-SPECIFIC DATA - WATERBORNE PATHWAYS via MISSOURI RIVER

Average River Flow

at Site: $31,000 \mathrm{ft}^{3} / \mathrm{s}$

Drinking Water:

None

Fish:

Edible Harvest: $5.0 \mathrm{E} 3(\mathrm{a}) \mathrm{kg} / \mathrm{yr}$

(a) Assumes 1/2 fish harvest caught below plant. 
POPULATION DOSE-COMMITMENT ESTIMATES AND

AVERAGE INDIVIDUAL DOSE-COMMITMENT HISTOGRAM FOR

COOPER

Dose Commitments (person-rem) from Waterborne Pathways

Total Body

GI-LLI

Ihyrotd

Bone

Liver

Infant

$0.0 E+00$

$0.0 E+00$

$0.0 E+00$

$0.0 \mathrm{E}+00$

$0.0 E+00$

Child

2. $9 \mathrm{E}-04$

2. $5 \mathrm{E}-05$

$1.8 \mathrm{E}-05$

$1.4 \mathrm{E}-03$

$1.6 \mathrm{E}-03$

Teen

5. $5 \mathrm{E}-04$

5. $2 \mathrm{E}-05$

1. $3 \mathrm{E}-05$

8.6E-04

$1.4 \mathrm{E}-03$

Adult

6. $0 \mathrm{E}-03$

4. 5E-04

8. $3 \mathrm{E}-05$

$5.0 \mathrm{E}-03$

8. $2 \mathrm{E}-03$

TOTAL

$6.8 \mathrm{E}-03$

5. $3 \mathrm{E}-04$

7. 3E-03

$1.1 \mathrm{E}-02$

Dose Commitments (person-rem) from Atrborne Pathways

Iotal Body GI-LLI Thyrotd Bone Liver Lung

$\begin{array}{lllllll}\text { Infant } & 7.2 \mathrm{E}-05 & 7.0 \mathrm{E}-05 & 1.4 \mathrm{E}-03 & 7.5 \mathrm{E}-05 & 7.4 \mathrm{E}-05 & 7.3 \mathrm{E}-05 \\ \text { Child } & 8.0 \mathrm{E}-04 & 7.9 \mathrm{E}-04 & 1.3 \mathrm{E}-02 & 9.6 \mathrm{E}-04 & 8.2 \mathrm{E}-04 & 8.2 \mathrm{E}-04 \\ \text { Teen } & 5.8 \mathrm{E}-04 & 5.8 \mathrm{E}-04 & 5.2 \mathrm{E}-03 & 6.2 \mathrm{E}-04 & 5.9 \mathrm{E}-04 & 6.2 \mathrm{E}-04 \\ \text { Adult } & 3.5 \mathrm{E}-03 & 3.5 \mathrm{E}-03 & 2.0 \mathrm{E}-02 & 3.6 \mathrm{E}-03 & 3.5 \mathrm{E}-03 & 3.6 \mathrm{E}-03 \\ \text { TOTAL } & 4.9 \mathrm{E}-03 & 4.9 \mathrm{E}-03 & 3.9 \mathrm{E}-02 & 5.3 \mathrm{E}-03 & 5.0 \mathrm{E}-03 & 5.1 \mathrm{E}-03\end{array}$

Production/Consumption factors:

Produce: 2.9 Milk: 3.2 Meat: 14

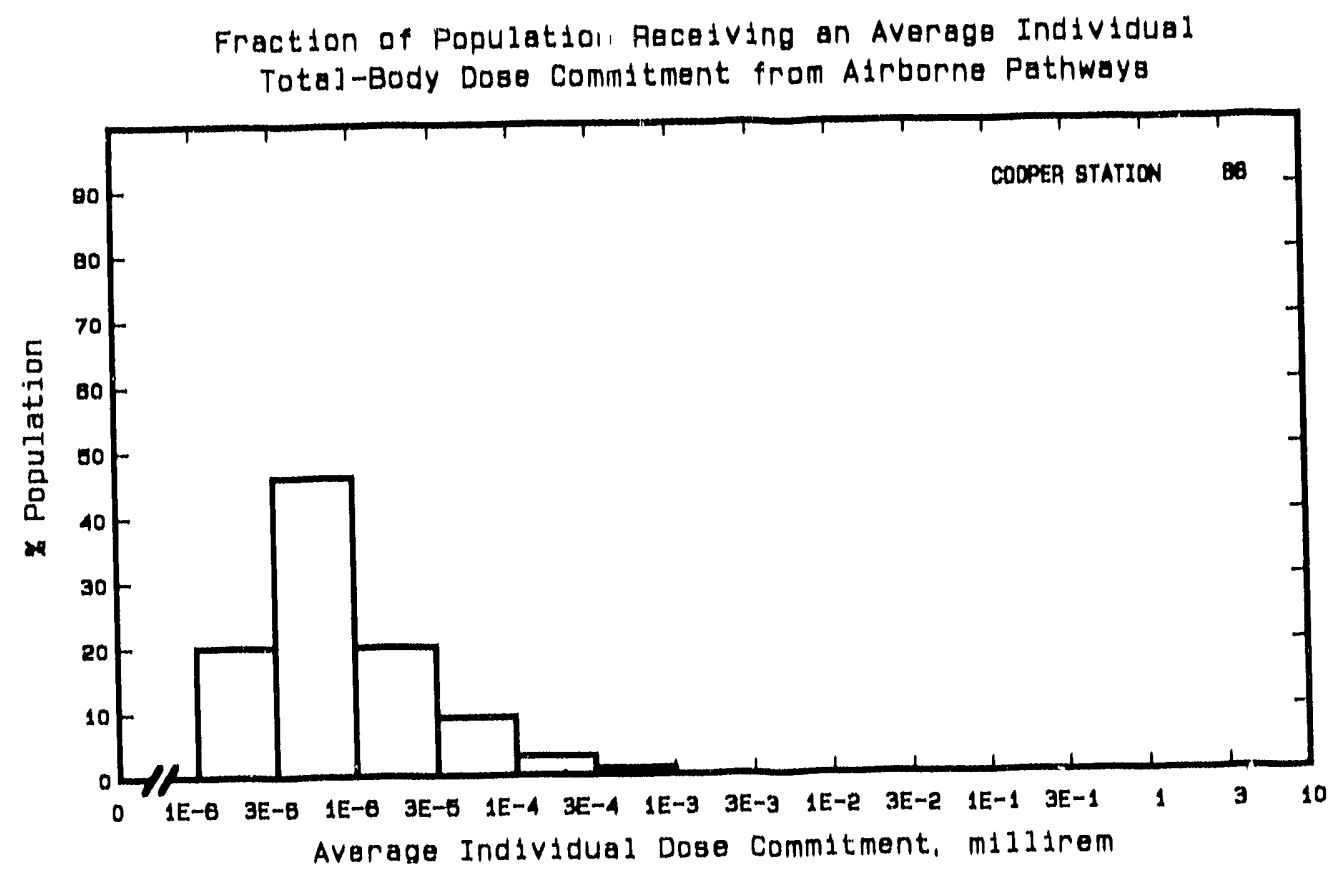


Site: CRYSTAL RIVER

CRYSTAL RIVER, FLORIDA

Location: $\quad N 28.9572^{\circ} \quad W 82.6989^{\circ}$

POPULATION DATA

Total Population Within 2-to-80-km Region: 4.6E5

Major Metropolitan Centers Within Region:

Center

Ocala

Leesburg

New Port Richey
Population

47,000

16,000

14,000
Location

$60 \mathrm{~km} \quad \mathrm{ENE}$

$80 \mathrm{~km} \quad \mathrm{E}$

$79 \mathrm{~km} \mathrm{~S}$

SITE-SPECIFIC DATA - AIRBORNE PATHWAYS

Average Annual State Production

of Crops and Animal Products

In $80-\mathrm{km}$ Radius Circle

Regional Productivity Factor:

Animal Grazing Factor:

Metenrology Period of Record: 1 JAN 75 - 31 DEC 75 Recovery: 93\%
Veg: $2.8 E 7$ kilogram

Milk: 1.1E8 liter

Meat: $7.2 E 7$ kilogram

0.5

1

SITE-SPECIFIC DATA - WATERBORNE PATHWAYS VIa GULF OF MEXICO

Fish:

Average Dilution Flow

from Plant: $94 \mathrm{ft}^{3} / \mathrm{s}$

Edible Harvest: $3.2 \mathrm{E} 5 \mathrm{~kg} / \mathrm{yr}$

Difution Factor: 0.1

Invertebrates:

Edible Harvest: $1.8 \mathrm{E} 5 \mathrm{~kg} / \mathrm{yr}$

Dilution Factor: 0.1 
POPULATION DOSE-COMMITMENT ESTIMATES AND

AVERAGE INDIVIDUAL DOSE-COMMITMENT HISTOGRAM FOR

CRYSTAL RIVER

Dose Commitments (person-rem) from Waterborne Pathways

Total Body GI-LLI Ihyroid Bone Liver

$\begin{array}{llllll}\text { Infant } & 0.0 \mathrm{E}+00 & 0.0 \mathrm{E}+00 & 0.0 \mathrm{E}+00 & 0.0 \mathrm{E}+00 & 0.0 \mathrm{E}+00 \\ \text { Child } & 2.8 \mathrm{E}-02 & 1.4 \mathrm{E}-01 & 1.2 \mathrm{E}-02 & 6.6 \mathrm{E}-02 & 4.6 \mathrm{E}-02 \\ \text { Teen } & 2.0 \mathrm{E}-02 & 2.8 \mathrm{E}-01 & 9.3 \mathrm{E}-03 & 3.7 \mathrm{E}-02 & 3.6 \mathrm{E}-02 \\ \text { Adult } & 1.4 \mathrm{E}-01 & 2.5 \mathrm{E}+00 & 6.5 \mathrm{E}-02 & 2.2 \mathrm{E}-01 & 2.2 \mathrm{E}-01 \\ \text { TOTAL } & 1.9 \mathrm{E}-01 & 2.9 \mathrm{E}+00 & 8.7 \mathrm{E}-02 & 3.2 \mathrm{E}-01 & 3.0 \mathrm{E}-01\end{array}$

Dose Commitments (person-rem) from Airborne Pathways

Total Body GI-LLI Thyroid Bone Liver Lung

$\begin{array}{lllllll}\text { Infant } & 1.6 \mathrm{E}-04 & 1.6 \mathrm{E}-04 & 6.1 \mathrm{E}-04 & 1.5 \mathrm{E}-04 & 1.6 \mathrm{E}-04 & 1.7 \mathrm{E}-04 \\ \text { Child } & 1.8 \mathrm{E}-03 & 1.8 \mathrm{E}-03 & 4.1 \mathrm{E}-03 & 1.7 \mathrm{E}-03 & 1.8 \mathrm{E}-03 & 2.0 \mathrm{E}-03 \\ \text { Teen } & 1.3 \mathrm{E}-03 & 1.3 \mathrm{E}-03 & 2.2 \mathrm{E}-03 & 1.2 \mathrm{E}-03 & 1.3 \mathrm{E}-03 & 1.5 \mathrm{E}-03 \\ \text { Adult } & 7.7 \mathrm{E}-03 & 7.7 \mathrm{E}-03 & 1.0 \mathrm{E}-02 & 7.2 \mathrm{E}-03 & 7.7 \mathrm{E}-03 & 8.5 \mathrm{E}-03 \\ \text { TOTAL } & 1.1 \mathrm{E}-02 & 1.1 \mathrm{E}-02 & 1.7 \mathrm{E}-02 & 1.0 \mathrm{E}-02 & 1.1 \mathrm{E}-02 & 1.2 \mathrm{E}-02\end{array}$

Production/Consumption factors:

Produce: $0.16 \quad$ Milk: 0.93 Meat: 0.98

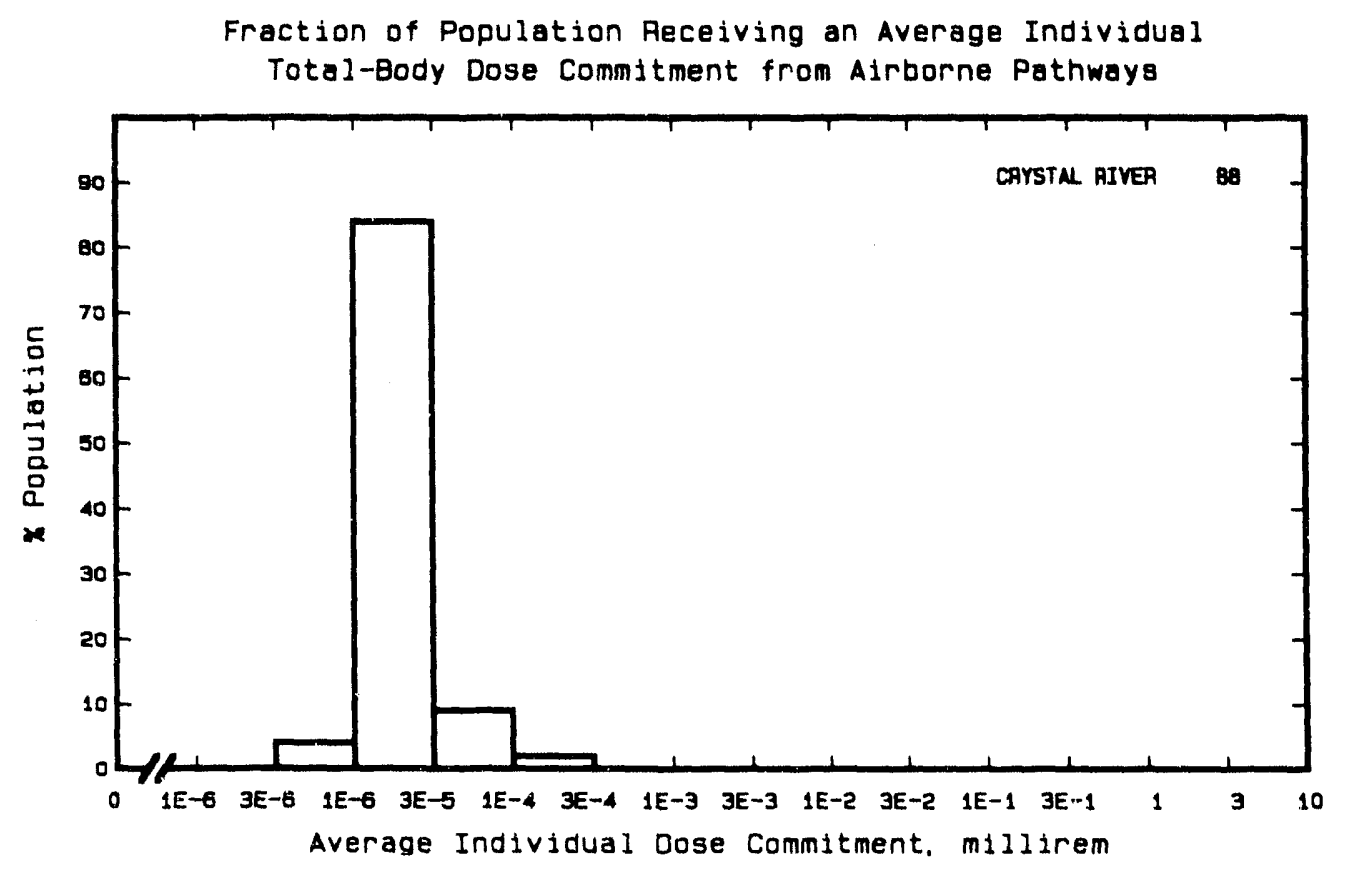


Site: DAVIS-BESSE

PORT CLINTON, OHIO

Location: $\quad N 41.5972^{\circ} \quad$ W $83.0864^{\circ}$

POPULATION DATA

Total Population Within 2-to-80-km Region: 1.8E6

Major Metropolitan Centers Within Region:

Center

Toledo SMSA

Dearborn

Taylor Lorain

Lincoln Park

Findlay

Sandusky
Population

610,000

91,000

77,000

75,000

45,000

36,000

33,000

\section{Location}

$38 \mathrm{~km} \quad W N W$

$80 \mathrm{~km} \mathrm{~N}$

$71 \mathrm{~km} \mathrm{~N}$

$77 \mathrm{~km} \quad \mathrm{ESE}$

$73 \mathrm{~km} \mathrm{~N}$

$77 \mathrm{~km} \quad \mathrm{SW}$

$35 \mathrm{~km} \quad \mathrm{ESE}$

SITE-SPECIFIC DATA - AIRBORNE PATHWAYS

Average Annual State Production

of Crops and Animal Products

In $80-\mathrm{km}$ Radius Circle

Regional Productivity Factor:

Animal Grazing Factor:
Veg: $6.9 E 7$ kilogram

Milk: 3.7E8 liter

Meat: $1.2 \mathrm{E} 8 \mathrm{kilogram}$

0.6

0.5

Meteorology Period of Record: 4 AUG 74 - 3 AUG 76 Recovery: $99 \%$

SITE-SPECIFIC DATA - WATERBORNE PATHWAYS via LAKE ERIE

Average Dilution Flow

from Plant: $330 \mathrm{ft}^{3} / \mathrm{s}$

Drinking Water:

Exposed Population: 450,000

Dilution Factor: $1.6 \mathrm{E}-4(\mathrm{a})$

Fish:

Edible Harvest: $5.7 \mathrm{E} 6$ (b) $\mathrm{kg} / \mathrm{yr}$

Dilution Factor: $1.8 \mathrm{E}-4$ (b)

(a) Drinking water dilution factor estimated by averaging dilution factor derived from FES (1973), suitably we ighted for population.

(b) Letter from Terry D. Murray, Toledo Edison Company to Charles A. Willis, NRC, July 20, 1984. 


\section{POPULATION DOSE-COMMITMENT ESTIMATES AND \\ AVERAGE. INDIVIDUAL DOSE-COMMITMENT HISTOGRAM FOR \\ DAVIS-BESSE}

Dose Commitments (person-rem) from Waterborne Pathways

Total Body GI-LLI Thyroid Bone Liver

$\begin{array}{llllll}\text { Infant } & 7.8 \mathrm{E}-06 & 8.5 \mathrm{E}-06 & 8.4 \mathrm{E}-06 & 1.6 \mathrm{E}-06 & 8.1 \mathrm{E}-06 \\ \text { Child } & 1.6 \mathrm{E}-04 & 1.4 \mathrm{E}-04 & 9.0 \mathrm{E}-05 & 3.3 \mathrm{E}-04 & 4.2 \mathrm{E}-04 \\ \text { Teen } & 1.5 \mathrm{E}-04 & 1.2 \mathrm{E}-04 & 3.5 \mathrm{E}-05 & 1.9 \mathrm{E}-04 & 3.1 \mathrm{E}-04 \\ \text { Adult } & 1.5 \mathrm{E}-03 & 1.1 \mathrm{E}-03 & 2.9 \mathrm{E}-04 & 1.1 \mathrm{E}-03 & 1.9 \mathrm{E}-03 \\ \text { TOTAL } & 1.8 \mathrm{E}-03 & 1.3 \mathrm{E}-03 & 4.2 \mathrm{E}-04 & 1.6 \mathrm{E}-03 & 2.7 \mathrm{E}-03\end{array}$

Dose Commitments (person-rem) from Airborne Pathways

Total Body GI-LLI Thyroid Bone Liver Lung

$\begin{array}{lllllll}\text { Infant } & 2.5 \mathrm{E}-04 & 2.5 \mathrm{E}-04 & 7.1 \mathrm{E}-04 & 2.8 \mathrm{E}-05 & 2.5 \mathrm{E}-04 & 2.5 \mathrm{E}-04 \\ \text { Child } & 3.1 \mathrm{E}-03 & 3.1 \mathrm{E}-03 & 5.7 \mathrm{E}-03 & 3.1 \mathrm{E}-04 & 3.1 \mathrm{E}-03 & 3.1 \mathrm{E}-03 \\ \text { Teen } & 1.9 \mathrm{E}-03 & 1.9 \mathrm{E}-03 & 2.9 \mathrm{E}-03 & 2.2 \mathrm{E}-04 & 1.9 \mathrm{E}-03 & 2.0 \mathrm{E}-03 \\ \text { Adult } & 1.0 \mathrm{E}-02 & 1.02 \mathrm{E}-02 & 1.3 \mathrm{E}-02 & 1.3 \mathrm{E}-03 & 1.0 \mathrm{E}-02 & 1.0 \mathrm{E}-02 \\ \text { TOTAL } & 1.6 \mathrm{E}-02 & 1.6 \mathrm{E}-02 & 2.3 \mathrm{E}-02 & 1.9 \mathrm{E}-03 & 1.6 \mathrm{E}-02 & 1.6 \mathrm{E}-02\end{array}$

Production/Consumption factors:

Produce: $0.12 \quad$ Milk: $0.95 \quad$ Meat: 0.48

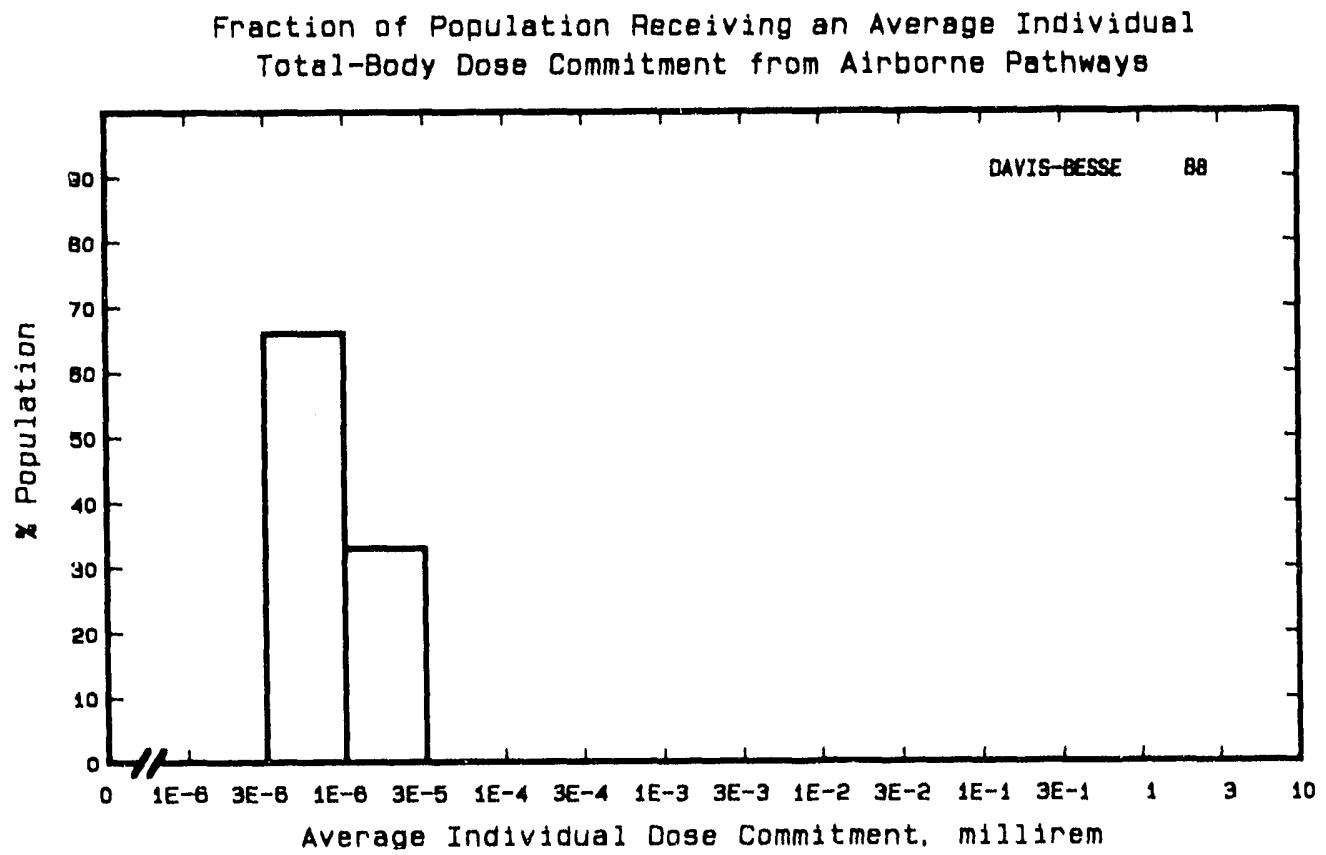


Site: DIABLO CANYON

AVILA BEACH, CALIFORNIA

Location: $\quad$ N $35.2111^{\circ} \quad$ W $120.8522^{\circ}$

POPULATION DATA

Total Population Within 2-to-80-km Region: 3.1 E5

Major Metropolitan Centers Within Region:

\begin{tabular}{lcccc} 
Center & Population & \multicolumn{3}{c}{ Location } \\
\cline { 2 - 6 } San Luis 0bispo & 40,000 & $19 \mathrm{~km}$ & ENE \\
Atascudero & 14,000 & $34 \mathrm{~km}$ & NNE \\
Lompoc & 28,000 & $74 \mathrm{~km}$ & SSE \\
Morro Bay & 10,000 & $18 \mathrm{~km}$ & $\mathrm{~N}$
\end{tabular}

SITE-SPECIFIC DATA - AIRBORNE PATHWAYS

Average Annual State Production

of Crops and Animal Products

In $80-\mathrm{km}$ Radius Circle

Regional Productivity Factor:

Animal Grazing Factor:

Meteorology Period of Record: I JAN 84 - 31 DEC 84 Recovery: 98\%
Veg: $4.8 E 7$ kilogram

Milk: 2.3E8 liter

Meat: $5.0 E 7$ kilogram

0.5

1

SITE-SPECIFIC DATA - WATERBORNE PATHWAYS via Pacific Ocean

Average Dilution Flow

from Plant: $720 \mathrm{ft}^{3} / \mathrm{s}$

Fish:

Edible Harvest: $2.0 \mathrm{E} 6 \mathrm{~kg} / \mathrm{yr}$ Dilution Factor: 0.001

Invertebrates: None 
POPULATION DOSE-COMMITMENT ESTIMATES AND

AVERAGE INDIVIDUAL DOSE-COMMITTMENT HISTOGRAM FOR

DIABLO CANYON 1 AND 2

Dose Commitments (person-rem) from Waterborne Pathways

Total Body GI-LLI Thyroid Bone Liver

$\begin{array}{llllll}\text { Infant } & 0.0 \mathrm{E}+00 & 0.0 \mathrm{E}+00 & 0.0 \mathrm{E}+00 & 0.0 \mathrm{E}+00 & 0.0 \mathrm{E}+00 \\ \text { Child } & 6.7 \mathrm{E}-04 & 5.9 \mathrm{E}-04 & 3.5 \mathrm{E}-04 & 2.8 \mathrm{E}-03 & 2.1 \mathrm{E}-03 \\ \text { Teen } & 6.1 \mathrm{E}-04 & 1.2 \mathrm{E}-03 & 2.6 \mathrm{E}-04 & 1.6 \mathrm{E}-03 & 1.7 \mathrm{E}-03 \\ \text { Adult } & 5.0 \mathrm{E}-03 & 9.9 \mathrm{E}-03 & 1.7 \mathrm{E}-03 & 9.3 \mathrm{E}-03 & 9.8 \mathrm{E}-03 \\ \text { TOTAL } & 6.3 \mathrm{E}-03 & 1.2 \mathrm{E}-02 & 2.3 \mathrm{E}-03 & 1.4 \mathrm{E}-02 & 1.4 \mathrm{E}-02\end{array}$

Dose Commitments (person-rem) from Airborne Pathways

Total Body GI-LLI Thyroid Bone Liver Lung

$\begin{array}{lllllll}\text { Infant } & 1.7 \mathrm{E}-04 & 1.7 \mathrm{E}-04 & 4.1 \mathrm{E}-04 & 5.0 \mathrm{E}-05 & 1.8 \mathrm{E}-04 & 1.8 \mathrm{E}-04 \\ \text { Child } & 2.7 \mathrm{E}-03 & 2.7 \mathrm{E}-03 & 4.1 \mathrm{E}-03 & 5.6 \mathrm{E}-04 & 2.7 \mathrm{E}-03 & 2.8 \mathrm{E}-03 \\ \text { Teen } & 1.8 \mathrm{E}-03 & 1.8 \mathrm{E}-03 & 2.3 \mathrm{E}-03 & 4.0 \mathrm{E}-04 & 1.8 \mathrm{E}-03 & 1.8 \mathrm{E}-03 \\ \text { Aduit } & 9.9 \mathrm{E}-03 & 9.9 \mathrm{E}-03 & 1.2 \mathrm{E}-02 & 2.4 \mathrm{E}-03 & 9.9 \mathrm{E}-03 & 1.0 \mathrm{E}-02 \\ \text { TOTAL } & 1.5 \mathrm{E}-02 & 1.5 \mathrm{E}-02 & 1.9 \mathrm{E}-02 & 3.4 \mathrm{E}-03 & 1.5 \mathrm{E}-02 & 1.5 \mathrm{E}-02\end{array}$

Production/Consumption factors:

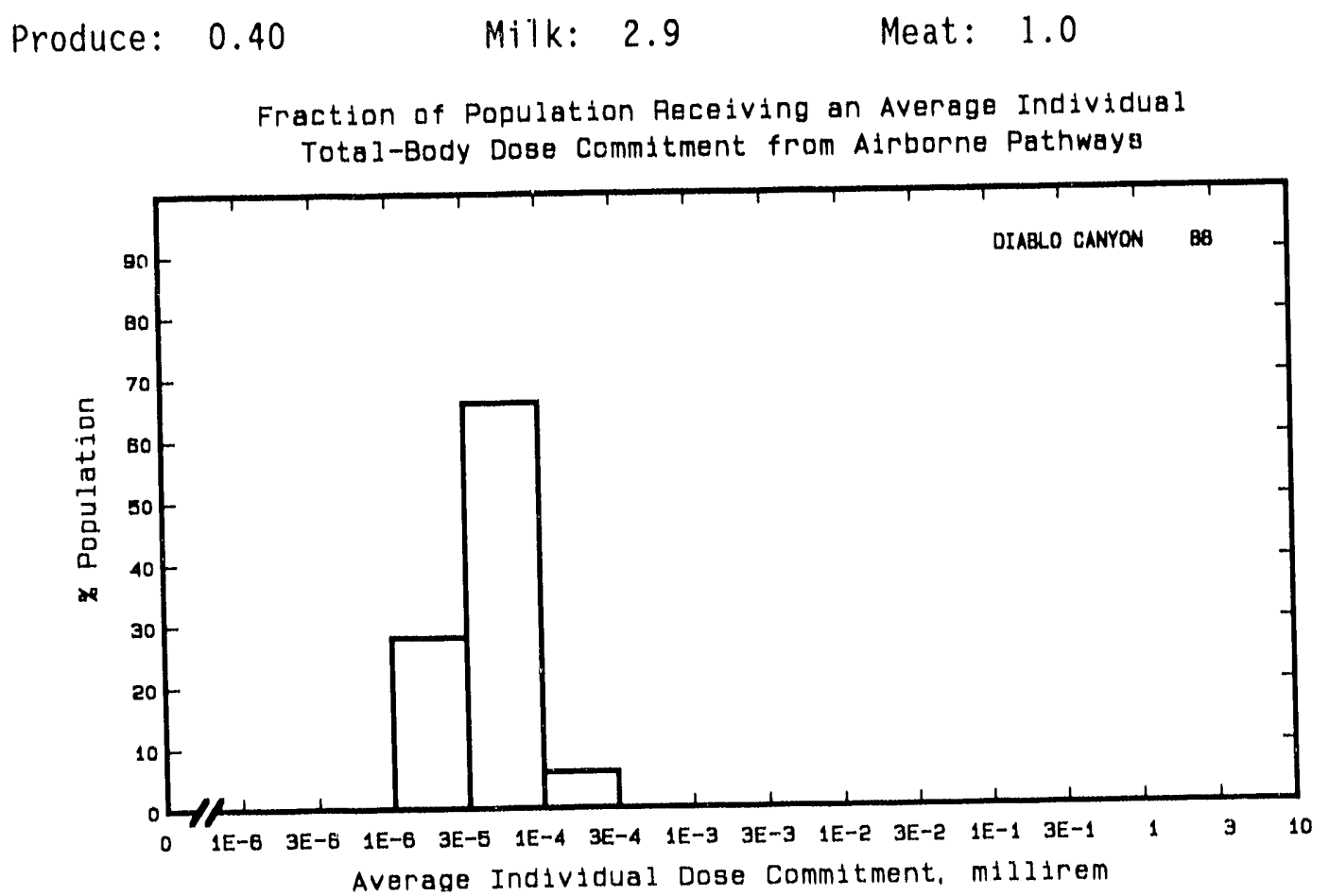


Site: DRESDEN

GRUNDY COUNTY, ILLINOIS

Location: $\quad$ N $41.3897^{\circ}$ W $88.2711^{\circ}$

POPULATION DATA

Total Population Within 2-to-80-km Region: 6.5E6

Major Metropolitan Centers Within Region:

Center

Chicago SMSA

Gary-Hammond-E. Chicago-SMSA

Kankakee SMSA

Aurora

Joliet

Elgin

\section{Population}

$7,200,000(a)$

$650,000(a)$

100,000

82,000

79,000

57,000
Location

$75 \mathrm{~km} \quad \mathrm{NE}$

$80 \mathrm{~km} \quad \mathrm{ENE}$

$45 \mathrm{~km} \quad \mathrm{SE}$

$41 \mathrm{~km} \mathrm{~N}$

$22 \mathrm{~km} \quad \mathrm{NE}$

$74 \mathrm{~km} \mathrm{~N}$

SITE-SPECIFIC DATA - AIRBORNE PATHWAYS

Average Annual State Production

of Crops and Animal Products

In $80-\mathrm{km}$ Radius Circle

Regional Productivity Factor:

Animal Grazing Factor:
Veg: $1.1 \mathrm{E8}$ kilogram

Milk: 1.8E8 1iter

Meat: $1.9 \mathrm{E} 8 \mathrm{kilogram}$

1

0.5

Meteorology Period of Record: 1 JAN 74 - 31 JAN 75 Recovery: $77 \%$

SITE-SPECIFIC DATA - WATERBORNE PATHWAYS via ILLINOIS RIVER

Average River Flow

at Site: $12,000 \mathrm{ft}^{3} / \mathrm{s}$

Drinking Water:

Exposed Population: (b)

Fish:

Edible Harvest: (b)

(a) Population of total SMSA given; population of SMSA fraction within $80 \mathrm{~km}$ of site would be somewhat smaller.

(b) River water used for sewage disposal for Chicago, so population doses from liquid pathways assumed to be near zero. 
POPULATION DOSE-COMMITMENT ESTIMATES AND

AVERAGE INDIVIDUAL DOSE-COMMITMENT HISTOGRAM FOR

DRESDEN 1, 2, AND 3

Dose Commitments (person-rem) from Waterborne Pathways

Total Body GI-LLI Thyroid Bone Liver

Infant

Child

Teen

Adult

(Little or No Waterborne Pathway Doses)

TOTAL

Dose Commitments (person-rem) from Airborne Pathways

Total Body GI-LLI Thyroid Bone Liver Lung

$\begin{array}{lllllll}\text { Infant } & 1.4 \mathrm{E}-03 & 1.3 \mathrm{E}-03 & 7.5 \mathrm{E}-02 & 1.3 \mathrm{E}-03 & 1.6 \mathrm{E}-03 & 1.8 \mathrm{E}-03 \\ \text { Child } & 1.8 \mathrm{E}-02 & 1.7 \mathrm{E}-02 & 6.3 \mathrm{E}-01 & 1.4 \mathrm{E}-02 & 1.9 \mathrm{E}-02 & 2.4 \mathrm{E}-02 \\ \text { Teen } & 1.3 \mathrm{E}-02 & 1.3 \mathrm{E}-02 & 3.0 \mathrm{E}-01 & 9.4 \mathrm{E}-03 & 1.3 \mathrm{E}-02 & 1.9 \mathrm{E}-02 \\ \text { Adult } & 7.4 \mathrm{E}-02 & 7.8 \mathrm{E}-02 & 1.2 \mathrm{E}+00 & 5.4 \mathrm{E}-02 & 7.6 \mathrm{E}-02 & 1.0 \mathrm{E}-01 \\ \text { TOTAL } & 1.1 \mathrm{E}-01 & 1.1 \mathrm{E}-01 & 2.2 \mathrm{E}+00 & 7.9 \mathrm{E}-02 & 1.1 \mathrm{E}-01 & 1.4 \mathrm{E}-01\end{array}$

Production/Consumption factors:

Produce: 0.090 Milk: 0.21 Meat: 0.36

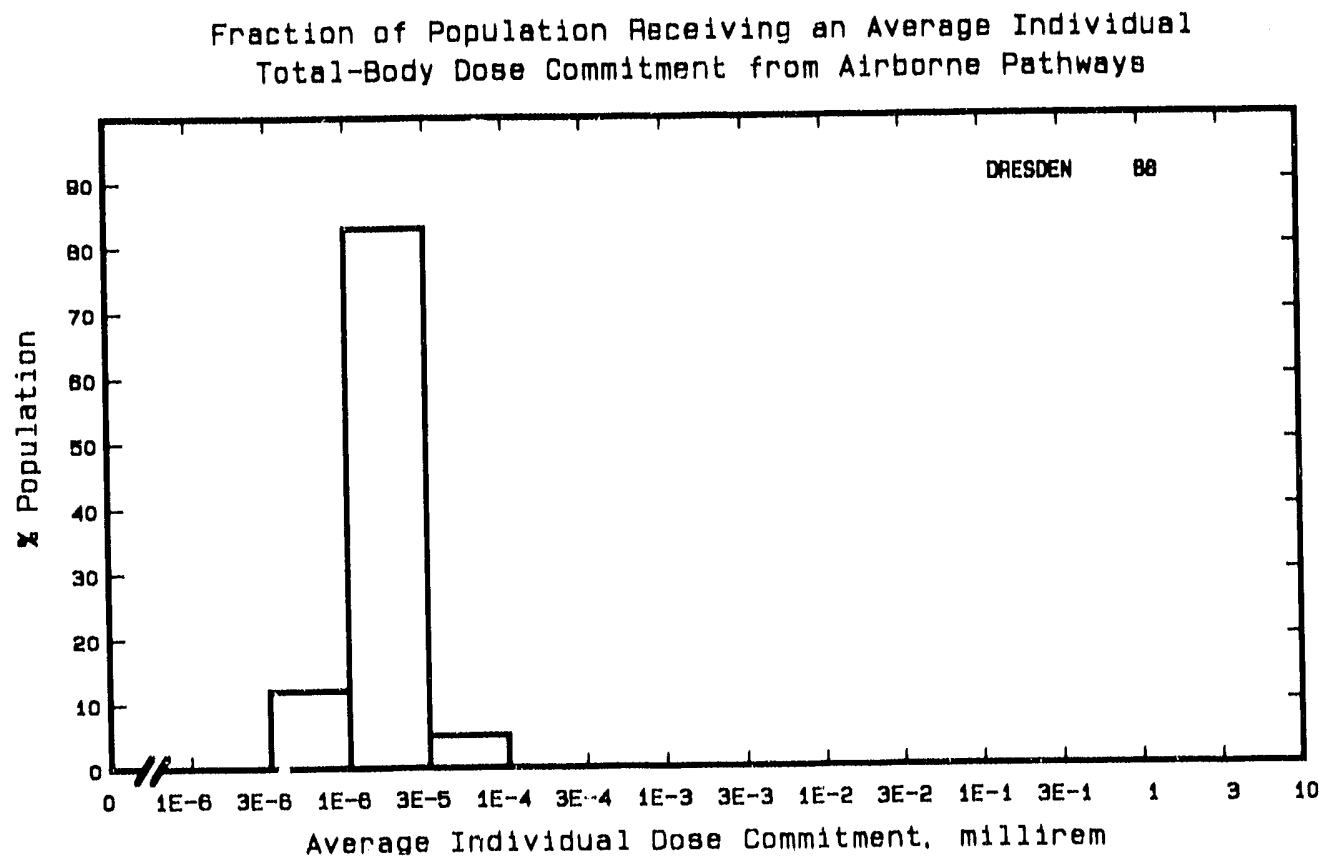


Site: DUANE ARNOLD

CEDAR RAPIDS, IOWA

Location: $\quad N \quad 42.1006^{\circ} \quad$ W $91.7772^{\circ}$

POPULATION DATA

Total Population Within 2-to-80-km Region: 5.8E5

Major Metropolitan Centers Within Region:

Center

Population

Location

Cedar Rapids SMSA

170,000

Water100-Cedar Falls SMSA 140,000

Iowa City SMSA

Marion

81,000

19,000

$17 \mathrm{~km} \quad \mathrm{SE}$

$64 \mathrm{~km} \quad \mathrm{NW}$

$52 \mathrm{~km}$ SSE

$16 \mathrm{~km} \quad \mathrm{ESE}$

SITE-SPECIFIC DATA - AIRBORNE PATHWAYS

Average Annual State Production

of Crops and Animal Products

In $80-\mathrm{km}$ Radius Circle

Veg: $9.8 \mathrm{E} 7 \mathrm{kilogram}$

Milk: 2.6E8 Titer

Meat: $4.2 E 8$ kilogram

Regional Productivity Factor:

1

Animal Grazing Factor:

0.5

Meteorology Period of Record: 1 FEB 71 - 31 DEC 75 Recovery: $57 \%$

SITE-SPECIFIC DATA - WATERBORNE PATHWAYS via CEDAR RIVER

Average River Flow

at Site: $3,100 \mathrm{ft}^{3} / \mathrm{s}$

Drinking Water: None

Fish: None 


\section{POPULATION DOSE-COMMITMENT ESTIMATES AND \\ AVERAGE INDIVIDUAL DOSE-COMMITMENT HISTOGRAM FOR \\ DUANE ARNOLD}

Dose Commitments (person-rem) from Waterborne Pathways

Total Body GI-LLI Ihyrotd Bone Liver

Infant

child

Teen

(No Waterborne Pathway Doses) (a)

Adult

TOTAL

Dose Commitments (person-rem) from Airborne Pathways

Total Body GI-LLI Thyroid Bone Liver Lung

$\begin{array}{lllllll}\text { Infant } & 8.9 \mathrm{E}-04 & 8.9 \mathrm{E}-04 & 3.1 \mathrm{E}-03 & 8.3 \mathrm{E}-04 & 9.0 \mathrm{E}-04 & 9.3 \mathrm{E}-04 \\ \text { Child } & 1.1 \mathrm{E}-02 & 1.1 \mathrm{E}-02 & 3.1 \mathrm{E}-02 & 9.3 \mathrm{E}-03 & 1.1 \mathrm{E}-02 & 1.1 \mathrm{E}-02 \\ \text { Teen } & 7.6 \mathrm{E}-03 & 7.7 \mathrm{E}-03 & 1.6 \mathrm{E}-02 & 6.7 \mathrm{E}-03 & 7.6 \mathrm{E}-03 & 8.3 \mathrm{E}-03 \\ \text { Adult } & 4.5 \mathrm{E}-02 & 4.6 \mathrm{E}-02 & 7.5 \mathrm{E}-02 & 4.1 \mathrm{E}-02 & 4.5 \mathrm{E}-02 & 4.8 \mathrm{E}-02 \\ \text { TOTAL } & 6.4 \mathrm{E}-02 & 6.5 \mathrm{E}-02 & 1.3 \mathrm{E}-01 & 5.7 \mathrm{E}-02 & 6.4 \mathrm{E}-02 & 6.9 \mathrm{E}-02\end{array}$

Production/Consumption factors:

Produce: 0.86

Milk: 3.3

Meat: 8.9

Fraction of Population Receiving an Average Individual

Total-Body Dose Commitment from Alrborne Pathways

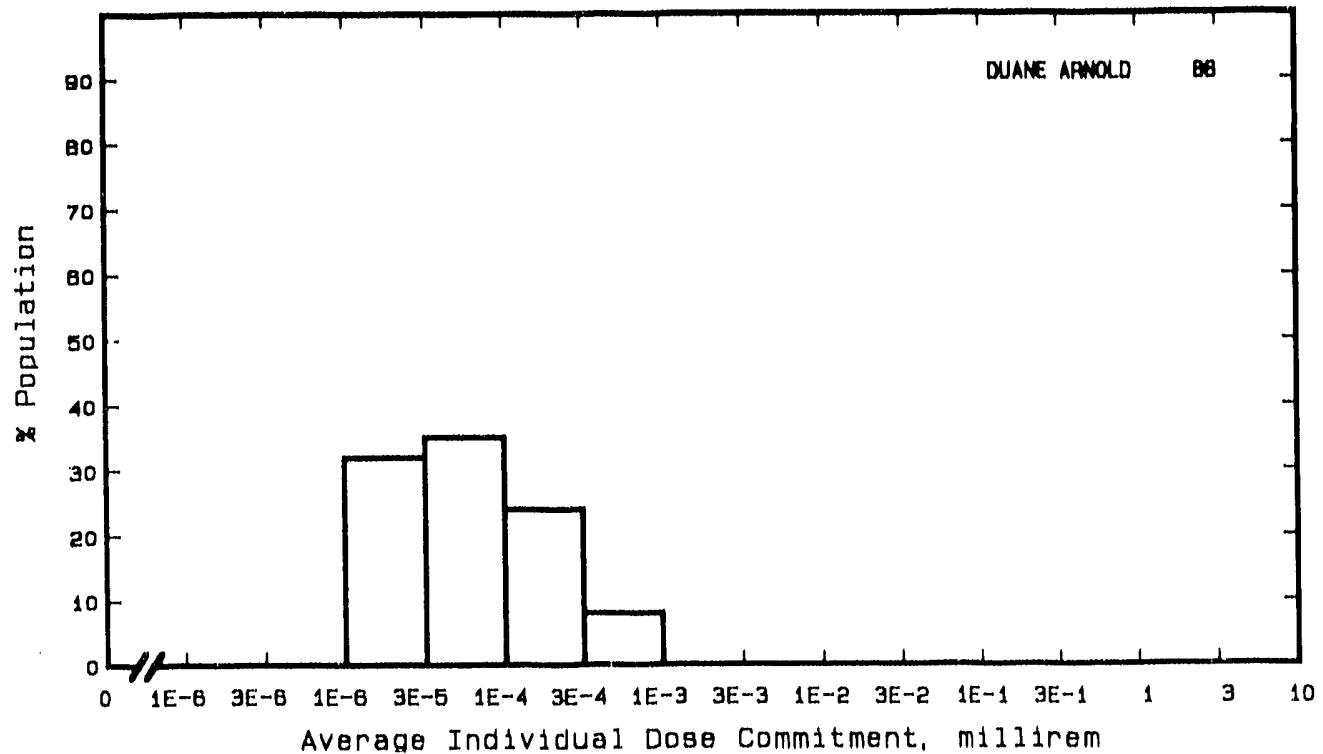

(a) No liquid releases reported (Tichler, et al. 1991). 
Stte: J. M. FARLEY

DOTHAN, ALABAMA

Location: $\quad$ N $31.2228^{\circ} \quad W 85.1126^{\circ}$

POPULATION DATA

Total Population Within 2-to-80-km Region: 3.7E.5

Major Metropolitan Centers Within Region:

\begin{tabular}{lccc}
\multicolumn{1}{c}{ Center } & Population & \multicolumn{2}{c}{ Location } \\
\cline { 2 - 5 } & & $27 \mathrm{~km}$ & $W$ \\
Dothan & 51,000 & $71 \mathrm{~km}$ & $W$ \\
Enterprise & 18,000 & $56 \mathrm{~km}$ & WNW \\
Ozark & 13,000 & $75 \mathrm{~km}$ & $\mathrm{~N}$ \\
Eufaula & 12,000 & $62 \mathrm{~km}$ & SE \\
Batnbridge & 11,000 &
\end{tabular}

Average Annual State Production

of Crops and Animal Products

In $80-\mathrm{km}$ Radius Circle

Regional Productivity Factor:

Animal Grazing Factor:
Veg: $1.7 \mathrm{E} 7 \mathrm{k \uparrow logram}$

Milk: 5.7E7 1tter

Meat: 8.6E7 ktlogram

0.95

0.8

Meteornlogy Period of Record: 1 APR 71 - 31 MAR 75 Recovery: 100\%

SITE-SPECIFIC DATA - WATERBORNE PATHWAYS VIa CHATTAHOOCHEE RIVER

Average River Flow

at Site: $12,000 \mathrm{ft}^{3} / \mathrm{s}$

Drinking Water:

None

Fish:

Edible Harvest: $2.3 \mathrm{E} 5 \mathrm{~kg} / \mathrm{yr}$

Dilution Factor: 1 
POPULATION DOSE-COMMITMENT ESTIMATES AND

AVERAGE INDIVIDUAL DOSE-COMMITMENT HISTOGRAM FOR

J. M. FARLEY 1 AND 2

Dose Commltments (person-rem) from Waterborne Pathways

Iotal Body GI-LLI Thyrotd Bone Liver

$\begin{array}{llllll}\text { Infant } & 0.0 \mathrm{E}+\mathrm{CO} & 0.0 \mathrm{E}+00 & 0.0 \mathrm{E}+00 & 0.0 \mathrm{E}+00 & 0.0 \mathrm{E}+00 \\ \text { Child } & 1.1 \mathrm{E}-03 & 3.5 \mathrm{E}-03 & 3.2 \mathrm{E}-04 & 4.3 \mathrm{E}-03 & 4.8 \mathrm{E}-03 \\ \text { Teen } & 1.7 \mathrm{E}-03 & 7.0 \mathrm{E}-03 & 2.9 \mathrm{E}-04 & 2.5 \mathrm{E}-03 & 4.0 \mathrm{E}-03 \\ \text { Adult } & 1.8 \mathrm{E}-02 & 6.0 \mathrm{E}-02 & 2.3 \mathrm{E}-03 & 1.5 \mathrm{E}-02 & 2.4 \mathrm{E}-02 \\ \text { TOTAL } & 2.0 \mathrm{E}-02 & 7.1 \mathrm{E}-02 & 2.9 \mathrm{E}-03 & 2.2 \mathrm{E}-02 & 3.3 \mathrm{E}-02\end{array}$

Dose Commttments (person-rem) from Atrborne Pathways

Total Body GI-LLI Thyrold Bone Liver Lung

$\begin{array}{lllllll}\text { Infant } & 5.9 \mathrm{E}-04 & 5.9 \mathrm{E}-04 & 9.4 \mathrm{E}-04 & 1.6 \mathrm{E}-04 & 5.9 \mathrm{E}-04 & 6.2 \mathrm{E}-04 \\ \text { Child } & 8.3 \mathrm{E}-03 & 8.3 \mathrm{E}-03 & 1.0 \mathrm{E}-02 & 1.8 \mathrm{E}-03 & 8.3 \mathrm{E}-03 & 8.7 \mathrm{E}-03 \\ \text { Teen } & 5.4 \mathrm{E}-03 & 5.4 \mathrm{E}-03 & 6.2 \mathrm{E}-03 & 1.3 \mathrm{E}-03 & 5.4 \mathrm{E}-03 & 5.9 \mathrm{E}-03 \\ \text { Adult } & 3.0 \mathrm{E}-02 & 3.0 \mathrm{E}-02 & 3.3 \mathrm{E}-02 & 7.8 \mathrm{E}-03 & 3.0 \mathrm{E}-02 & 3.2 \mathrm{E}-02 \\ \text { TOTAL } & 4.5 \mathrm{E}-02 & 4.5 \mathrm{E}-02 & 5.1 \mathrm{E}-02 & 1.1 \mathrm{E}-02 & 4.5 \mathrm{E}-02 & 4.7 \mathrm{E}-02\end{array}$

Production/Consumption factors:

Produce: $0.22 \quad$ Milk: $1.1 \quad$ Meat: 2.7

Fraction of Population Faceiving an Average Individual Total-Body Dose Cummitment from Alrborne Pathwaya

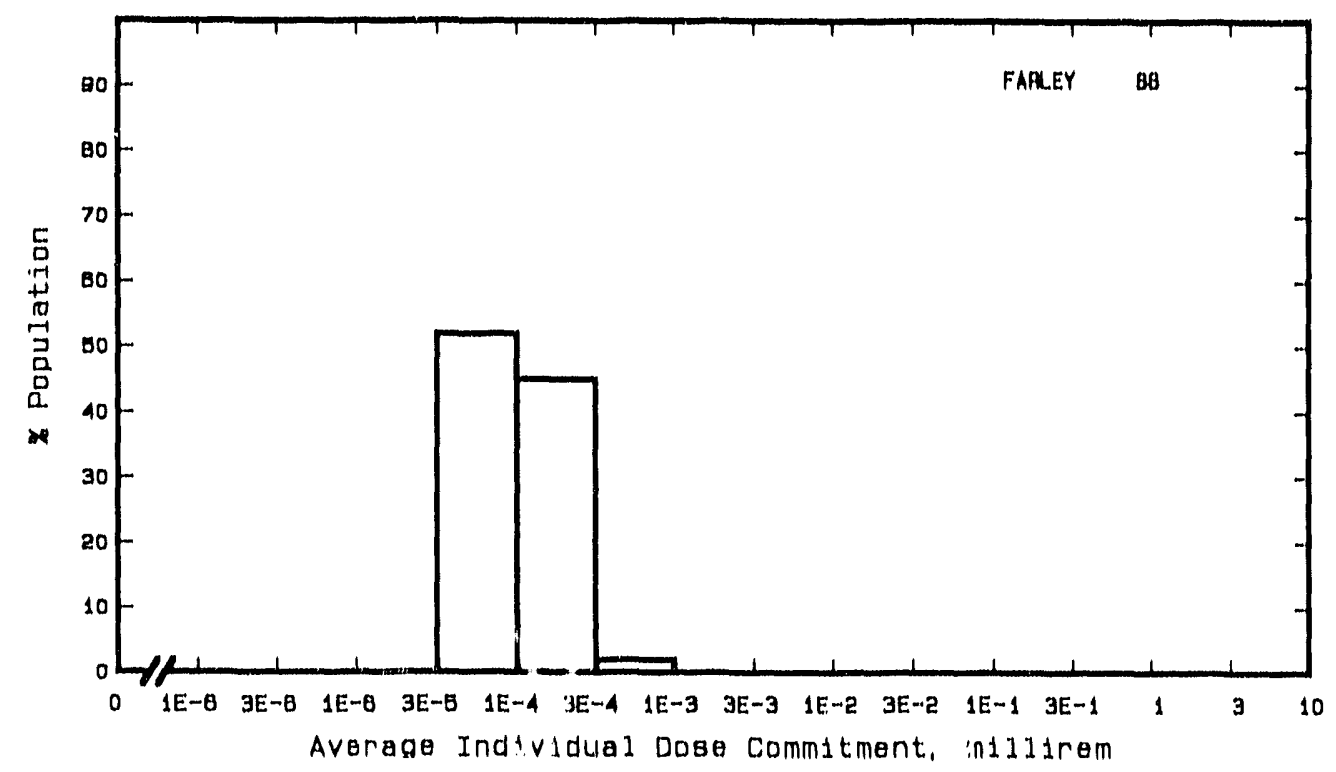


Stte: FERMI

LAGOONA BEACH, MICHIGAN

Location: $\quad$ N $41.9781^{\circ} \quad$ W $83.2594^{\circ}$

POPULATION DATA

Total Population Within 2-to-80-km Region: $4.9 E 6$

Major Metropolitan Centers Within Region:

Center

Detroit

Toledo

Ann Arbor

Sandusky

Monroe
Population

$1,200,000$
350,000
110,000
31,000
26,000

\section{Location}

24-80 km NNW

$40 \mathrm{~km} \quad \mathrm{SW}$

$48 \mathrm{~km} \quad \mathrm{NW}$

$72 \mathrm{~km} \quad \mathrm{SE}$

$13 \mathrm{~km}$ WSW

SITE-SPECIFIC DATA - AIRBORNE PATHWAYS

Average Annual State Production

of Crops and Animal Products

In $80-\mathrm{km}$ Radius Circle

Regional Productivity Factor:

Animal Grazing Factor:
Veg: $6.8 \mathrm{E} 7 \mathrm{kilogram}$

Milk: 2.9E8 itter

Meat: $4.5 E 7$ kilogram

0.6

0.5

Meteorology Pertod of Record: 1 JUN 74 - 31 MAY 75 Recovery: 90\%

Average Dilution Flow

from Plant: $44 \mathrm{ft}^{3} / \mathrm{s}$

Drinking Water:

Exposed Population: 520,000

Dilution Factor: 0.00056

Fish:
Edtble Harvest: $8.9 \mathrm{E} 6 \mathrm{~kg} / \mathrm{yr}$

Dilution Factor: 0.001 
POPULATION DOSE-COMMITMENT ESTIMATES AND

AVERAGE INDIVIDUAL DOSE-COMMITMENT HISTOGRAM FOR

FERMI 2

Dose Commitments (person-rem) from Waterborne Pathways

Iotal Body GI-LLI Ihyroid Bone Liver

$\begin{array}{llllll}\text { Infant } & 1.1 \mathrm{E}-05 & 2.0 \mathrm{E}-05 & 8.8 \mathrm{E}-06 & 1.7 \mathrm{E}-05 & 1.3 \mathrm{E}-05 \\ \text { Child } & 1.8 \mathrm{E}-03 & 2.4 \mathrm{E}-03 & 8.7 \mathrm{E}-05 & 1.3 \mathrm{E}-03 & 2.8 \mathrm{E}-03 \\ \text { Teen } & 1.2 \mathrm{E}-03 & 4.7 \mathrm{E}-03 & 3.1 \mathrm{E}-05 & 8.5 \mathrm{E}-04 & 2.6 \mathrm{E}-03 \\ \text { Adu1t } & 7.3 \mathrm{E}-03 & 4.2 \mathrm{E}-02 & 2.5 \mathrm{E}-04 & 5.7 \mathrm{E}-03 & 1.6 \mathrm{E}-02 \\ \text { TOTAL } & 1.0 \mathrm{E}-02 & 4.9 \mathrm{E}-02 & 3.8 \mathrm{E}-04 & 7.9 \mathrm{E}-03 & 2.1 \mathrm{E}-02\end{array}$

Dose Commitments (person-rem) from Atrborne Pathways

Iotal Body GI-LLI Ihyrotd Bone Liver Lung

$\begin{array}{lllllll}\text { Infant } & 2.9 \mathrm{E}-05 & 2.8 \mathrm{E}-05 & 7.7 \mathrm{E}-04 & 9.2 \mathrm{E}-05 & 3.0 \mathrm{E}-05 & 6.6 \mathrm{E}-05 \\ \text { Child } & 4.8 \mathrm{E}-04 & 3.3 \mathrm{E}-04 & 7.6 \mathrm{E}-03 & 2.3 \mathrm{E}-03 & 3.2 \mathrm{E}-04 & 9.1 \mathrm{E}-04 \\ \text { Teen } & 3.0 \mathrm{E}-04 & 3.0 \mathrm{E}-04 & 4.2 \mathrm{E}-03 & 1.6 \mathrm{E}-03 & 2.2 \mathrm{E}-04 & 7.4 \mathrm{E}-04 \\ \text { Adult } & 1.6 \mathrm{E}-03 & 1.7 \mathrm{E}-03 & 1.9 \mathrm{E}-02 & 8.3 \mathrm{E}-03 & 1.1 \mathrm{E}-03 & 3.1 \mathrm{E}-03 \\ \text { TOTAL } & 2.4 \mathrm{E}-03 & 2.3 \mathrm{E}-03 & 3.1 \mathrm{E}-02 & 1.2 \mathrm{E}-02 & 1.7 \mathrm{E}-03 & 4.9 \mathrm{E}-03\end{array}$

Production/Consumption factors:

Produce: $0.043 \quad$ Milk: 0.27 Meat: 0.068

Fraction of Population Facalving an Average Individual
Total-Body Dose Commdtment from Adroorne Pathwaya

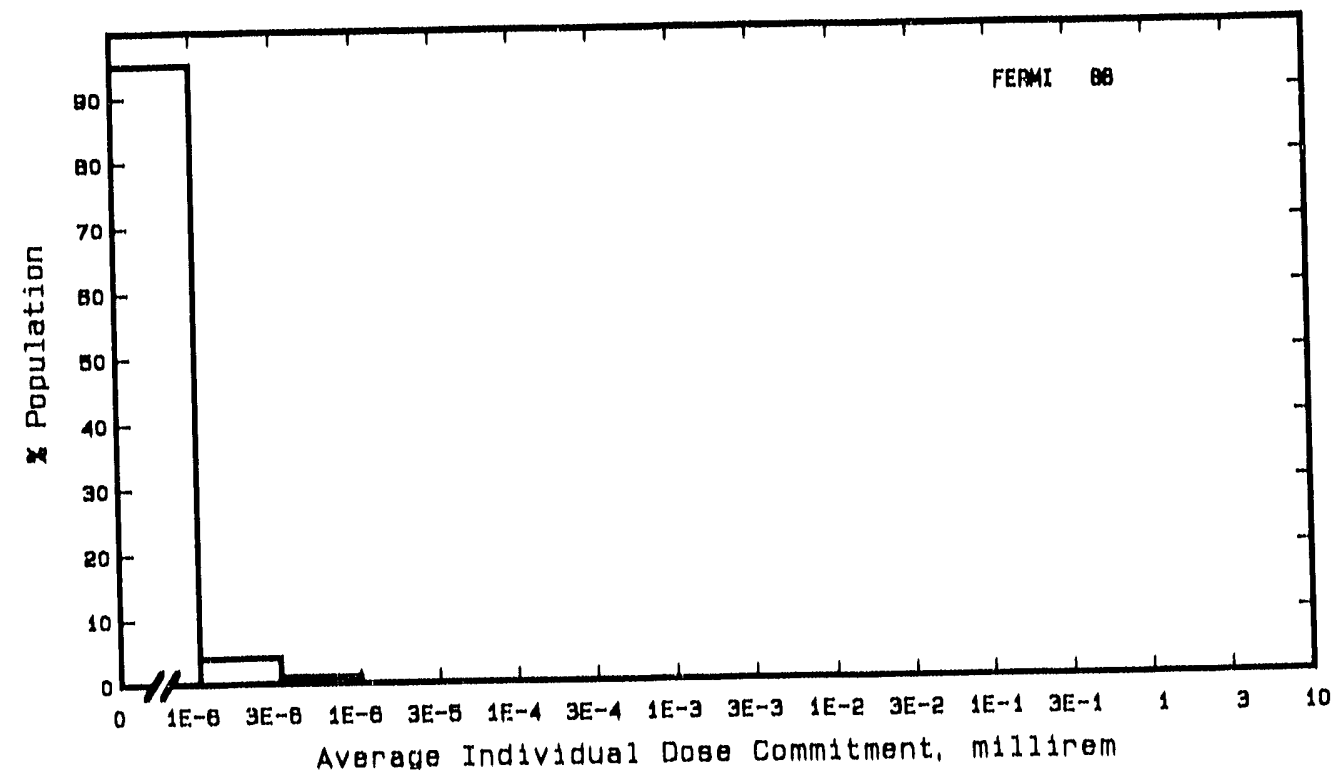


Site: J. A. FITZPATRICK

OSWEGO, NEW YORK

Location: $\quad$ N $43.5239^{\circ} \quad$ W $76.3983^{\circ}$

POPULATION DATA

Total Population Within 2-to-80-km Region: 8.6 E5

Major Metropolitan Centers Within Region:

\begin{tabular}{lccc}
\multicolumn{1}{c}{ Center } & Population & \multicolumn{2}{c}{ Locatton } \\
\cline { 2 - 4 } & & & \\
Syracuse SMSA & 650,000 & $86 \mathrm{~km}$ & SSE \\
Rome & 48,000 & $60 \mathrm{~km}$ & ESE \\
Auburn & 33,000 & $66 \mathrm{~km}$ & SSW \\
Watertown & 28,000 & $64 \mathrm{~km}$ & $\mathrm{NE}$ \\
Kingston & 24,000 & $79 \mathrm{~km}$ & $\mathrm{~N}$
\end{tabular}

SITE-SPECIFIC DATA - AIRBORNE PATHWAYS

Average Annual State Production

Veg: $7.6 \mathrm{E} 7$ kilogram

of Crops and Animal Products

Milk: 7.0E8 11ter

In $80-\mathrm{km}$ Radius Circle

Meat: $3.3 E 7$ kilogram

Regional Productivity Factor:

Antmal Grazing Factor:

0.7

0.5

Meteorology Period of Record: 1 JAN 74 - 31 DEC 75 Recovery: $97 \%$

SITE-SPECIFIC DATA - WATERBORNE PATHWAYS via LAKE ONTARIO
Average Dllution Flow
fron Plant: $710 \mathrm{ft}^{3} / \mathrm{s}$
Drinking Water:
Exposed Population: $549,000(a)$
Dilution Factor: $0.003(b)$
Fish:
Edible Harvest: $7.355 \mathrm{~kg} / \mathrm{yr}$
Dilution Factor: $0.005 \mathrm{~B})$

(a) Population exposed to drinking water derived from Nine Mlle Point FES (1974).

(b) Dilution factors dertved from FES (1973). 


\title{
POPULATION DOSE-COMMITMENT ESTIMATES AND \\ AVERAGE INDIVIDUAL DOSE-COMMITMENT HISTOGRAM FOR
}

\author{
J. A. FITZPATRICK
}

Dose Commitments (person-rem) from Waterborne Pathways

Total Body GI-LLI Thyroid Bone Liver

$\begin{array}{llllll}\text { Infant } & 2.6 \mathrm{E}-05 & 2.1 \mathrm{E}-05 & 6.7 \mathrm{E}-05 & 4.8 \mathrm{E}-05 & 8.3 \mathrm{E}-05 \\ \text { Child } & 9.5 \mathrm{E}-04 & 3.1 \mathrm{E}-04 & 5.5 \mathrm{E}-04 & 3.8 \mathrm{E}-03 & 4.5 \mathrm{E}-03 \\ \text { Teen } & 1.4 \mathrm{E}-03 & 2.4 \mathrm{E}-04 & 1.9 \mathrm{E}-04 & 2.1 \mathrm{E}-03 & 3.4 \mathrm{E}-03 \\ \text { Adult } & 1.5 \mathrm{E}-02 & 2.1 \mathrm{E}-03 & 1.4 \mathrm{E}-03 & 1.2 \mathrm{E}-02 & 2.02 \mathrm{E}-02 \\ \text { TOTAL } & 1.7 \mathrm{E}-02 & 2.7 \mathrm{E}-03 & 2.2 \mathrm{E}-03 & 1.8 \mathrm{E}-02 & 2.8 \mathrm{E}-02\end{array}$

Dose Commitments (person-rem) from Airborne Pathways

Total Body GI-LLI Thyroid Bone Liver Lung

$\begin{array}{lllllll}\text { Infant } & 2.1 \mathrm{E}-03 & 2.1 \mathrm{E}-03 & 1.3 \mathrm{E}-02 & 2.1 \mathrm{E}-03 & 2.1 \mathrm{E}-03 & 2.1 \mathrm{E}-03 \\ \text { Child } & 2.3 \mathrm{E}-02 & 2.3 \mathrm{E}-02 & 9.3 \mathrm{E}-02 & 2.3 \mathrm{E}-02 & 2.3 \mathrm{E}-02 & 2.4 \mathrm{E}-02 \\ \text { Teen } & 1.7 \mathrm{E}-02 & 1.7 \mathrm{E}-02 & 4.3 \mathrm{E}-02 & 1.7 \mathrm{E}-02 & 1.7 \mathrm{E}-02 & 1.8 \mathrm{E}-02 \\ \text { Adult } & 1.0 \mathrm{E}-01 & 1.0 \mathrm{E}-01 & 1.9 \mathrm{E}-01 & 1.0 \mathrm{E}-01 & 1.0 \mathrm{E}-01 & 1.1 \mathrm{E}-01 \\ \text { TOTAL } & 1.4 \mathrm{E}-01 & 1.4 \mathrm{E}-01 & 3.3 \mathrm{E}-01 & 1.4 \mathrm{E}-01 & 1.4 \mathrm{E}-01 & 1.5 \mathrm{E}-01\end{array}$

Production/Consumption factors:

Produce: 0.32 Milk: 4.4 Meat: 0.34

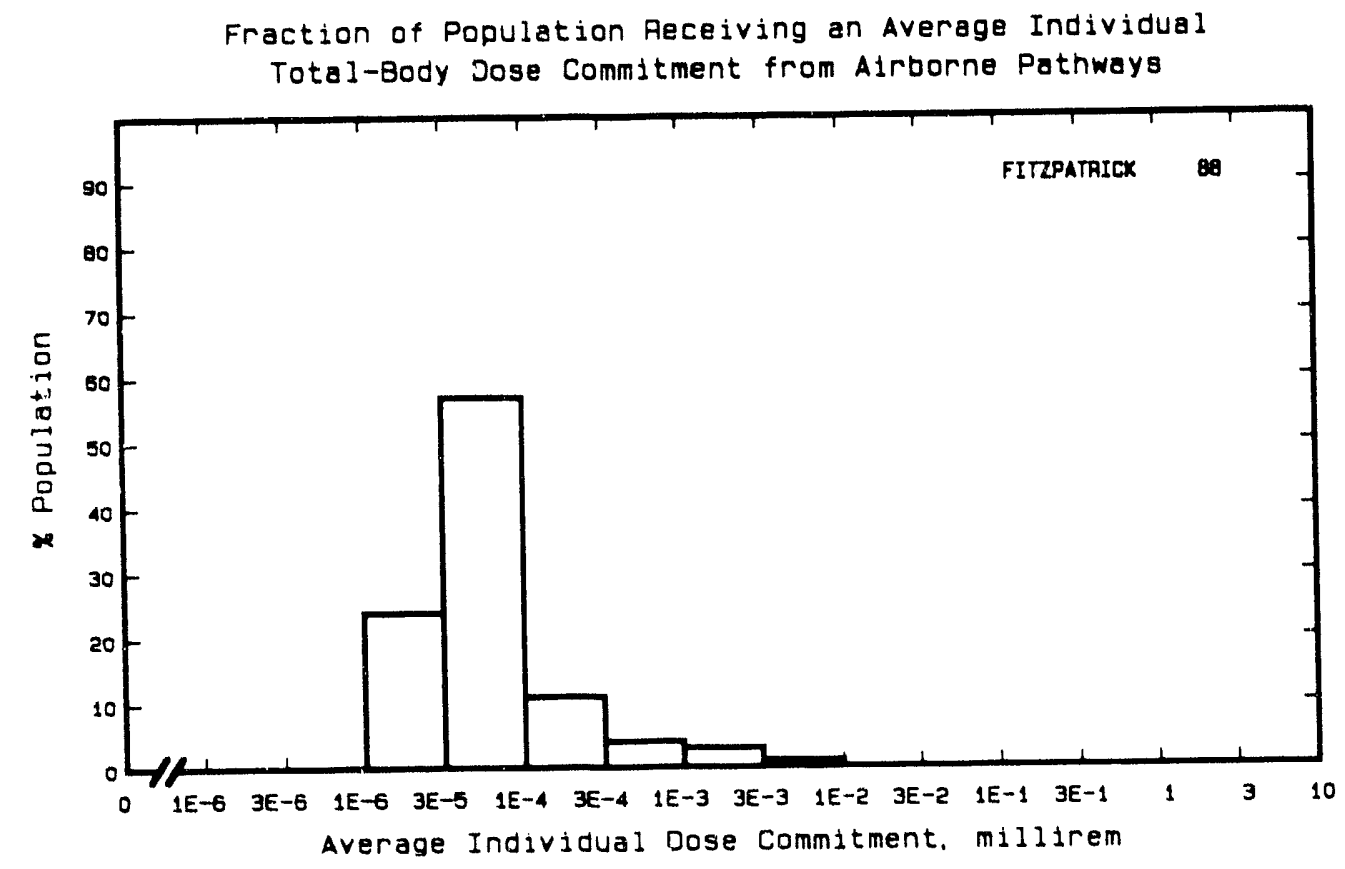


Site: FORT CALHOUN

WASHINGTON COUNTY, NEBRASKA

Location: $\quad$ N $41.5208^{\circ} \quad$ W $96.0767^{\circ}$

POPULATION DATA

Total Population Within 2-to-80-km Region: 7.6 E5

Major Metropolitan Centers Within Region:

Center
Omaha SMSA
Council BIuffs
Freemont
Bellevue

Population

Location

610,000

56,000

24,000

22,000

$\begin{array}{lll}32 & \mathrm{~km} & \text { SSE } \\ 34 & \mathrm{~km} & \text { SE } \\ 36 & \mathrm{~km} & \text { WSW } \\ 44 & \mathrm{~km} & \text { SSE }\end{array}$

SITE-SPECIFIC DATA - AIRBORNE PATHWAYS

Average Annual State Production

of Crops and Animal Products

In $80-\mathrm{km}$ Radius Circle

Regional Productivity Factor:

Animal Grazing Factor:
Veg: $9.7 E 7$ kilogram

Milk: $7.2 E 7$ liter

Meat: 2.068 kilogram

1

0.5

Meteorology Period of Record: 1 JAN 74 - 31 DEC 74 Recovery: $98 \%$

\section{SITE-SPECIFIC DATA - WATERBORNE PATHWAYS via MISSOURI RIVER}

Average River Flow

$$
\text { at Site: } 27,000 \mathrm{ft}^{3} / \mathrm{s}
$$

Drinking Water:

Exposed Population: 580,000 (a)

Dilution Factor: 1

Fish:

Edible Harvest: $1.0 \mathrm{E} 4 \mathrm{~kg} / \mathrm{yr}$

Dilution Factor: 1

(a) Drinking water population assumed to be approximately Omaha SMSA (FES 1972). 


\section{POPULATION DOSE-COMMITMENT ESTIMATES AND \\ AVERAGE INDIVIDUAL DOSE-COMMITMENT HISTOGRAM FOR}

FORT CALHOUN

Dose Commitments (person-rem) from Waterborne Pathways

Total Body GI-LLI Ihyroid Bone Liver

$\begin{array}{llllll}\text { Infant } & 4.8 \mathrm{E}-03 & 4.4 \mathrm{E}-03 & 2.2 \mathrm{E}-02 & 4.9 \mathrm{E}-03 & 9.4 \mathrm{E}-03 \\ \text { Child } & 5.8 \mathrm{E}-02 & 5.0 \mathrm{E}-02 & 1.7 \mathrm{E}-01 & 5.5 \mathrm{E}-02 & 9.5 \mathrm{E}-02 \\ \text { Teen } & 2.6 \mathrm{E}-02 & 2.1 \mathrm{E}-02 & 5.5 \mathrm{E}-02 & 1.5 \mathrm{E}-02 & 3.6 \mathrm{E}-02 \\ \text { Adult } & 2.4 \mathrm{E}-01 & 1.8 \mathrm{E}-01 & 4.2 \mathrm{E}-01 & 9.9 \mathrm{E}-02 & 2.7 \mathrm{E}-01 \\ \text { TOTAL } & 3.3 \mathrm{E}-01 & 2.5 \mathrm{E}-01 & 6.6 \mathrm{E}-01 & 1.7 \mathrm{E}-01 & 4.1 \mathrm{E}-01\end{array}$

Dose Conmitments (person-rem) from Airborne Pathways

Total Body GI-LLI Thyroid Bone Liver Lung

$\begin{array}{lllllll}\text { Infant } & 1.1 \mathrm{E}-04 & 1.1 \mathrm{E}-04 & 2.4 \mathrm{E}-04 & 1.0 \mathrm{E}-04 & 1.1 \mathrm{E}-04 & 1.2 \mathrm{E}-04 \\ \text { Child } & 1.3 \mathrm{E}-03 & 1.3 \mathrm{E}-03 & 2.5 \mathrm{E}-03 & 1.2 \mathrm{E}-03 & 1.3 \mathrm{E}-03 & 1.4 \mathrm{E}-03 \\ \text { Teen } & 9.3 \mathrm{E}-04 & 9.3 \mathrm{E}-04 & 1.4 \mathrm{E}-03 & 8.4 \mathrm{E}-04 & 9.4 \mathrm{E}-04 & 1.1 \mathrm{E}-03 \\ \text { Adult } & 5.6 \mathrm{E}-03 & 5.6 \mathrm{E}-03 & 7.4 \mathrm{E}-03 & 5.1 \mathrm{E}-03 & 5.6 \mathrm{E}-03 & 6.2 \mathrm{E}-03 \\ \text { TOTAL } & 8.0 \mathrm{E}-03 & 7.9 \mathrm{E}-03 & 1.2 \mathrm{E}-02 & 7.2 \mathrm{E}-03 & 8.0 \mathrm{E}-03 & 8.9 \mathrm{E}-03\end{array}$

Production/Consumption factors:

Produce: 0.65 Milk: 0.72 Meat: 3.2

Fraction of Population Receiving an Average Individual

Total-Body Dose Commitment from Airborne Pathways

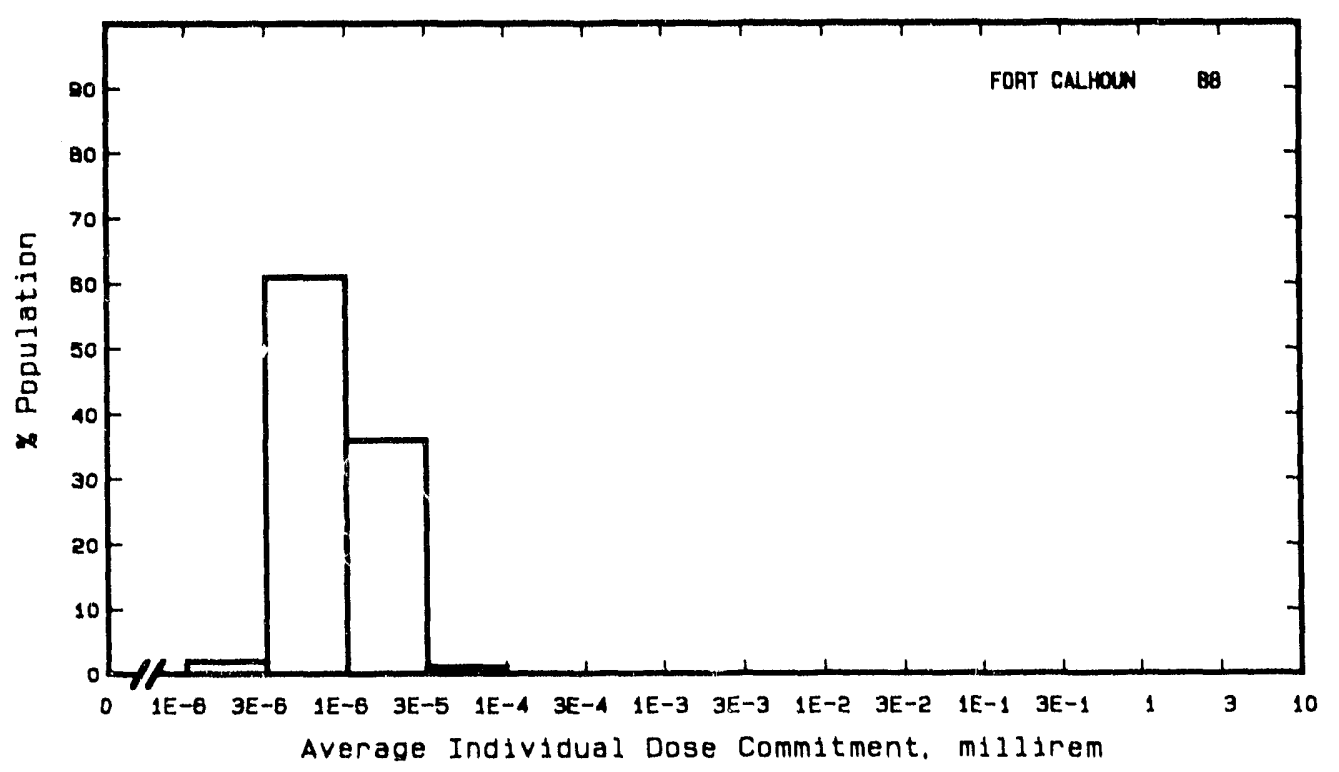


Site: R. E. GINNA

ONTARIO, NEW YORK

Location: $\quad$ N $43.2778^{\circ} \quad$ W $77.3089^{\circ}$

POPULATION DATA

Total Population Within 2-to-80-km Region: $1.2 \mathrm{E} 6$

Major Metropolitan Centers Within Region:

Center

Rochester SMSA

Auburn

Oswego

Batavia

Geneva
Population

990,000

32,000

20,000

17,000

15,000
Location

$27 \mathrm{~km} \quad W S W$

$71 \mathrm{~km} \quad$ ESE

$67 \mathrm{~km}$ ENE

$78 \mathrm{~km}$ WSW

$52 \mathrm{~km}$ SSE

SITE-SPECIFIC DATA - AIRBORNE PATHWAYS

Average Annual State Production

of Crops and Animal Products

In $80-\mathrm{km}$ Radius Circle

Regional Productivity Factor:

Animal Grazing Factor:

Meteorology Period of Record: 1 JAN 66 - 31 DEC 67 Recovery: 89\%
Veg: $7.6 \mathrm{E} 7 \mathrm{kilogram}$

Milk: 7.0E8 liter

Meat: $3.3 \mathrm{E} 7$ kilogram

0.6

0.5

SITE-SPECIFIC DATA - WATERBORNE PATHWAYS via LAKE ONTARIO

Average Dilution Flow

from Plant: $690 \mathrm{ft}^{3} / \mathrm{s}$

Drinking Water:

Exposed Population: 570,000

Dilution Factor: 0.01 (a)

Fish:

Edible Harvest: $7.3 \mathrm{E} 5 \mathrm{~kg} / \mathrm{yr}$

(a) Dilution factors from FES (1973). 


\section{POPULATION DOSE-COMMITMENT ESTIMATES AND \\ AVERAGE INDIVIDUAL DOSE-COMMITMENT HISTOGRAM FOR}

R. E. GINNA

Dose Commitments (person-rem) from Waterborne Pathways

Total Body GI-LLI Ihyroid Bone Liver

$\begin{array}{llllll}\text { Infant } & 2.5 \mathrm{E}-03 & 2.4 \mathrm{E}-03 & 5.5 \mathrm{E}-03 & 1.6 \mathrm{E}-04 & 2.6 \mathrm{E}-03 \\ \text { Child } & 2.9 \mathrm{E}-02 & 2.7 \mathrm{E}-02 & 4.9 \mathrm{E}-02 & 6.7 \mathrm{E}-03 & 3.4 \mathrm{E}-02 \\ \text { Teen } & 1.3 \mathrm{E}-02 & 1.1 \mathrm{E}-02 & 1.7 \mathrm{E}-02 & 3.5 \mathrm{E}-03 & 1.6 \mathrm{E}-02 \\ \text { Adult } & 1.1 \mathrm{E}-01 & 9.0 \mathrm{E}-02 & 1.4 \mathrm{E}-01 & 2.0 \mathrm{E}-02 & 1.2 \mathrm{E}-01 \\ \text { TOTAL } & 1.6 \mathrm{E}-01 & 1.3 \mathrm{E}-01 & 2.1 \mathrm{E}-01 & 3.1 \mathrm{E}-02 & 1.7 \mathrm{E}-01\end{array}$

Dose Commitments (person-rem) from Airborne Pathways

Total Body GI-LLI Thyroid Bone Liver Lung

$\begin{array}{lllllll}\text { Infant } & 2.1 \mathrm{E}-03 & 2.1 \mathrm{E}-03 & 2.2 \mathrm{E}-03 & 6.8 \mathrm{E}-03 & 2.1 \mathrm{E}-03 & 2.1 \mathrm{E}-03 \\ \text { Child } & 2.1 \mathrm{E}-02 & 2.1 \mathrm{E}-02 & 2.1 \mathrm{E}-02 & 5.8 \mathrm{E}-02 & 2.1 \mathrm{E}-02 & 2.1 \mathrm{E}-02 \\ \text { Teen } & 9.2 \mathrm{E}-03 & 9.2 \mathrm{E}-03 & 9.4 \mathrm{E}-03 & 1.8 \mathrm{E}-02 & 9.2 \mathrm{E}-03 & 9.2 \mathrm{E}-03 \\ \text { Aduit } & 4.0 \mathrm{E}-02 & 4.0 \mathrm{E}-02 & 4.1 \mathrm{E}-02 & 5.5 \mathrm{E}-02 & 4.02 \mathrm{E}-02 & 4.0 \mathrm{E}-02 \\ \text { TOTAL } & 7.3 \mathrm{E}-02 & 7.3 \mathrm{E}-02 & 7.4 \mathrm{E}-02 & 1.4 \mathrm{E}-01 & 7.3 \mathrm{E}-02 & 7.3 \mathrm{E}-02\end{array}$

Production/Consumption factors:

Produce: 0.19 Milk: 2.6 Meat: 0.20

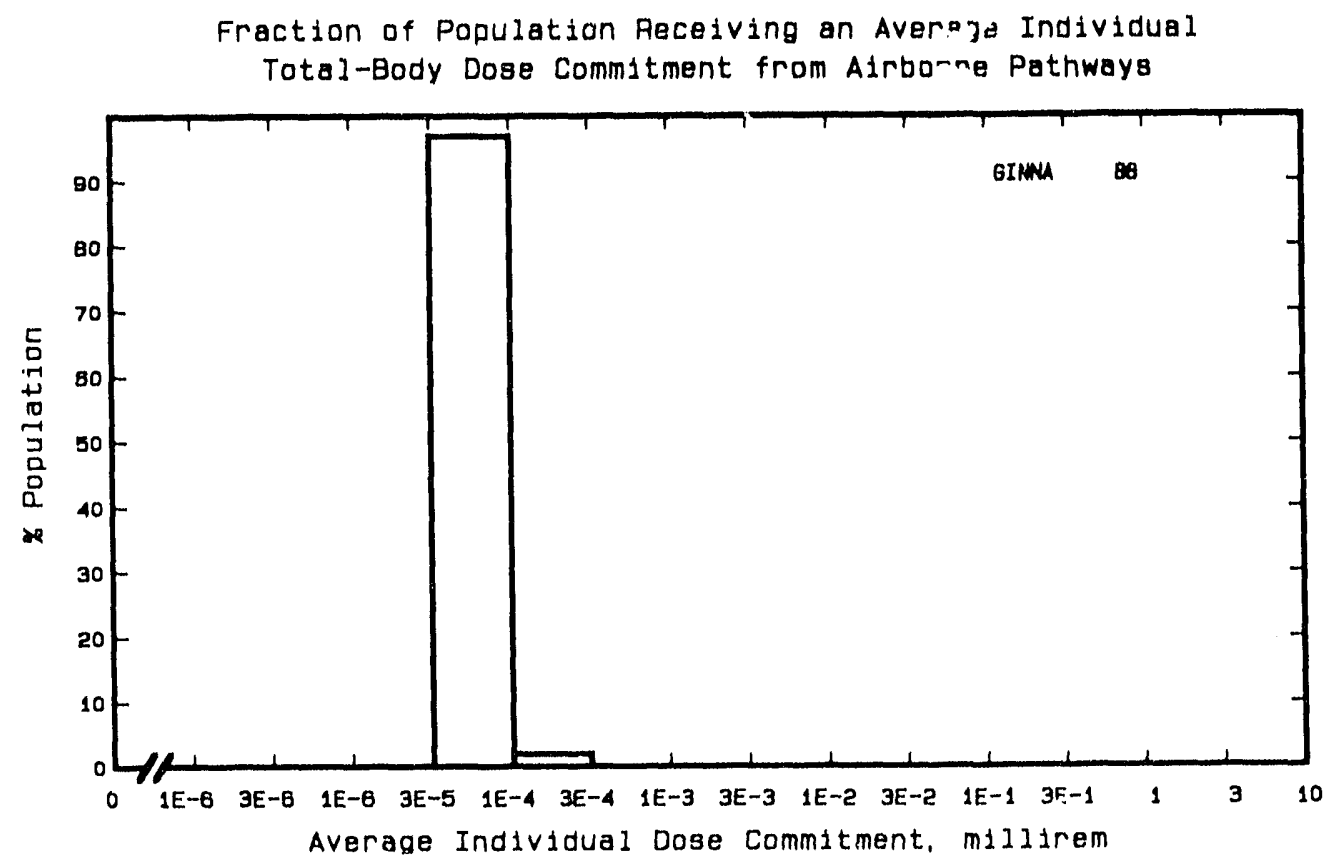


Site: GRAND GULF

PORT GIBSON, MISSISSIPPI

Location: $\quad N 32.0270^{\circ} \quad W 91.2530^{\circ}$

POPULATION DATA

Total Population Within 2-to-80-km Region: 3.4E5

Major Metropolitan Centers Within Region:

Center

Vicksburg

TaTlutah

Natches

Brookhaven
Population

27,000

10,000

20,000

11,000
Location

$40 \mathrm{~km} \quad \mathrm{NNE}$

$45 \mathrm{~km} \quad \mathrm{NNW}$

$60 \mathrm{~km} \quad \mathrm{SSW}$

$76 \mathrm{~km} \quad \mathrm{SE}$

SITE-SPECIFIC DATA - AIRBORNE PATHWAYS

Average Annual State Production

of Crops and Animal Products

In $80-\mathrm{km}$ Radius Circle

Regional Productivity Factor:

Animal Grazing Factor:
Veg: 4.4E6 kilogram

Milk: $7.1 E 7$ liter

Meat: $9.9 E 7$ kilogram

0.9

0.8

Meteorology Period of Record: 1 AUG 72 - 31 JUL 73 Recovery: 99\%

Average River

Flow at Site: $4000,000 \mathrm{ft}^{3} / \mathrm{s}$

Drinking Water: None

Fish:

Invertebrates:
Edible Harvest: $7.0 \mathrm{E} 5 \mathrm{~kg} / \mathrm{yr}$

Dilution Factor: 1

Edible Harvest: $7.0 \mathrm{E} 3 \mathrm{~kg} / \mathrm{yr}$

Dilution Factor: 1 
POPULATION DOSE-COMMITMENT ESTIMATES AND

AVERAGE INDIVIDUAL DOSE-COMMITMENT HISTOGRAM FOR

GRAND GULF

Dose Commitments (person-rem) from Waterborne Pathways

Total Body GI-LLI Ihyroid Bone Liver

$\begin{array}{llllll}\text { Infant } & 0.0 \mathrm{E}+00 & 0.0 \mathrm{E}+00 & 0.0 \mathrm{E}+00 & 0.0 \mathrm{E}+00 & 0.0 \mathrm{E}+00 \\ \text { Child } & 2.6 \mathrm{E}-05 & 5.4 \mathrm{E}-05 & 3.9 \mathrm{E}-07 & 5.3 \mathrm{E}-05 & 1.1 \mathrm{E}-04 \\ \text { Teen } & 3.2 \mathrm{E}-05 & 1.2 \mathrm{E}-04 & 3.3 \mathrm{E}-07 & 3.2 \mathrm{E}-05 & 9.4 \mathrm{E}-05 \\ \text { Adult } & 2.9 \mathrm{E}-04 & 1.1 \mathrm{E}-03 & 2.5 \mathrm{E}-06 & 1.9 \mathrm{E}-04 & 5.7 \mathrm{E}-04 \\ \text { TOTAL } & 3.5 \mathrm{E}-04 & 1.3 \mathrm{E}-03 & 3.3 \mathrm{E}-06 & 2.7 \mathrm{E}-04 & 7.7 \mathrm{E}-04\end{array}$

Dose Commitments (person-rem) frnm Airborne Pathways

Total Body GI-LLI Thyroid Bone Liver Lung

$\begin{array}{lllllll}\text { Infant } & 1.0 \mathrm{E}-05 & 1.0 \mathrm{E}-05 & 2.9 \mathrm{E}-05 & 3.7 \mathrm{E}-06 & 1.0 \mathrm{E}-05 & 1.1 \mathrm{E}-05 \\ \text { Child } & 1.3 \mathrm{E}-04 & 1.4 \mathrm{E}-04 & 2.3 \mathrm{E}-04 & 4.2 \mathrm{E}-05 & 1.3 \mathrm{E}-04 & 1.3 \mathrm{E}-04 \\ \text { Teen } & 8.5 \mathrm{E}-05 & 9.8 \mathrm{E}-05 & 1.3 \mathrm{E}-04 & 3.0 \mathrm{E}-05 & 8.5 \mathrm{E}-05 & 9.1 \mathrm{E}-05 \\ \text { Adult } & 4.9 \mathrm{E}-04 & 6.1 \mathrm{E}-04 & 6.3 \mathrm{E}-04 & 1.8 \mathrm{E}-04 & 4.9 \mathrm{E}-04 & 5.1 \mathrm{E}-04 \\ \text { TOTAL } & 7.1 \mathrm{E}-04 & 8.6 \mathrm{E}-04 & 1.0 \mathrm{E}-03 & 2.6 \mathrm{E}-04 & 7.1 \mathrm{E}-04 & 7.5 \mathrm{E}-04\end{array}$

Production/Consumption factors:

Produce: 0.060 Milk: $1.5 \quad$ Meat: 3.3

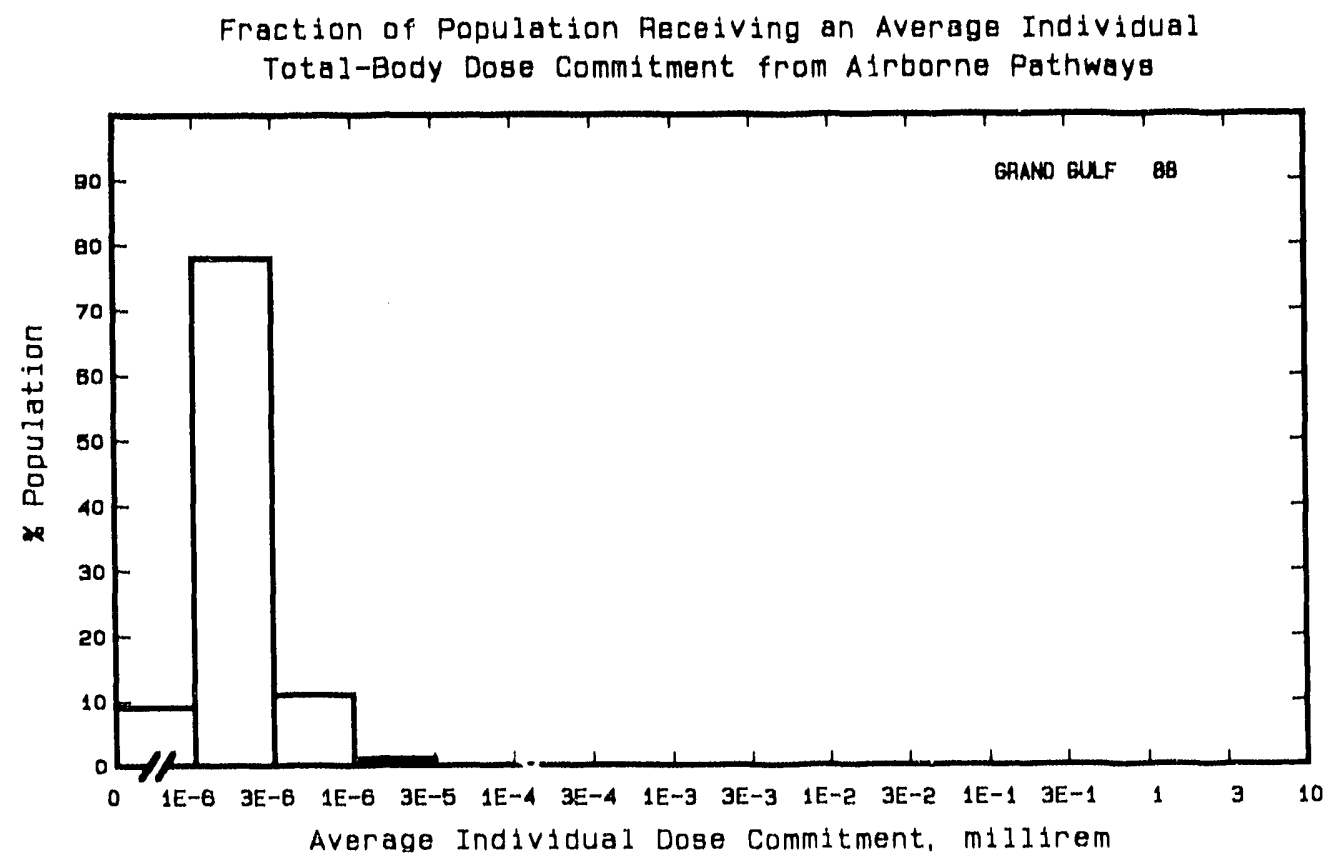


Site: HADDAM NECK

Location: $\quad$ N $41.4819^{\circ}$
HADDAM NECK, CONNECTICUT

W $72.4992^{\circ}$

POPULATION DATA

Total Population Within 2-to-80-km Region: 3.5E6

Major Metropolitan Centers Within Region:

Center

Hartford SMSA

Springfield-Chicopee-Holyoke SMSA

New Haven-West Haven SMSA

Bridgeport SMSA

New London-Norwich
Population

750,000

550,000

440,000

420,000

250,000
Location

$35 \mathrm{~km} \quad \mathrm{NNW}$

$70 \mathrm{~km} \mathrm{~N}$

$40 \mathrm{~km}$ WSW

$66 \mathrm{~km}$ WSW

$35 \mathrm{~km} \quad \mathrm{ESE}$

SITE-SPECIFIC DATA - AIRBORNE PATHWAYS

Average Annual State Production

of Crops and Animal Products

In $80-\mathrm{km}$ Radius Circle

Regional Productivity Factor:

Animal Grazing Factor:

Meteorology Period of Record: 1 JAN 75 - 31 DEC 75 Recovery: 95\%
Veg: $3.2 E 7 \mathrm{k \uparrow logram}$

Milk: 4.4E8 liter

Meat: $2.0 E 7$ kilogram

0.7

0.6

SITE-SPECIFIC DATA - WATERBORNE PATHWAYS via DISCHARGE CANAL TO CONN

RIVER

Average Discharge Canal

Flow at Site: $690 \mathrm{ft}^{3} / \mathrm{s}$

Drinking Water:

None

Fish:

Edible Harvest: $9.1 \mathrm{E}^{(\mathrm{a})} \mathrm{kg} / \mathrm{yr}$

Dilution Factor: 1

(a) Caught in discharge canal according to FES (1973). 


\begin{abstract}
POPULATION DOSE-COMMITMENT ESTIMATES AND
AVERAGE INDIVIDUAL DOSE-COMMITMENT HISTOGRAM FOR

HADDAM NECK
\end{abstract}

Dose Commitments (person-rem) from Waterborne Pathways

Total Body GI-LLI Thyrotd Bone Litver

$\begin{array}{llllll}\text { Infant } & 0.0 \mathrm{E}+00 & 0.0 \mathrm{E}+00 & 0.0 \mathrm{E}+00 & 0.0 \mathrm{E}+00 & 0.0 \mathrm{E}+00 \\ \text { Child } & 3.5 \mathrm{E}-03 & 4.8 \mathrm{E}-04 & 1.8 \mathrm{E}-03 & 1.8 \mathrm{E}-02 & 1.9 \mathrm{E}-02 \\ \text { Teen } & 6.3 \mathrm{E}-03 & 7.6 \mathrm{E}-04 & 1.3 \mathrm{E}-03 & 1.1 \mathrm{E}-02 & 1.6 \mathrm{E}-02 \\ \text { Adult } & 6.8 \mathrm{E}-02 & 6.4 \mathrm{E}-03 & 8.8 \mathrm{E}-03 & 6.3 \mathrm{E}-02 & 9.4 \mathrm{E}-02 \\ \text { TOTAL } & 7.7 \mathrm{E}-02 & 7.6 \mathrm{E}-03 & 1.2 \mathrm{E}-02 & 9.1 \mathrm{E}-02 & 1.3 \mathrm{E}-01\end{array}$

Dose Commitments (person-rem) from Airborne Pathways

Total Body GI-LLI Thyroid Bone Liver Lung

$\begin{array}{lllllll}\text { Infant } & 6.5 \mathrm{E}-03 & 6.5 \mathrm{E}-03 & 7.2 \mathrm{E}-02 & 4.4 \mathrm{E}-03 & 6.7 \mathrm{E}-03 & 6.9 \mathrm{E}-03 \\ \text { Child } & 7.7 \mathrm{E}-02 & 7.6 \mathrm{E}-02 & 4.5 \mathrm{E}-01 & 4.9 \mathrm{E}-02 & 7.7 \mathrm{E}-02 & 8.2 \mathrm{E}-02 \\ \text { Teen } & 5.5 \mathrm{E}-02 & 5.4 \mathrm{E}-02 & 2.2 \mathrm{E}-01 & 3.5 \mathrm{E}-02 & 5.5 \mathrm{E}-02 & 6.2 \mathrm{E}-02 \\ \text { Adult } & 3.2 \mathrm{E}-01 & 3.2 \mathrm{E}-01 & 9.2 \mathrm{E}-01 & 2.1 \mathrm{E}-01 & 3.2 \mathrm{E}-01 & 3.4 \mathrm{E}-01 \\ \text { TOTAL } & 4.6 \mathrm{E}-01 & 4.5 \mathrm{E}-01 & 1.7 \mathrm{E}+00 & 3.0 \mathrm{E}-01 & 4.6 \mathrm{E}-01 & 4.9 \mathrm{E}-01\end{array}$

Production/Consumption factors:

Produce: 0.033 Milk: 0.67 Meat: 0.051

Fraction of Population Receiving an Average Individual

Total-Body Dose Commitment from Alrborne Pathwaya

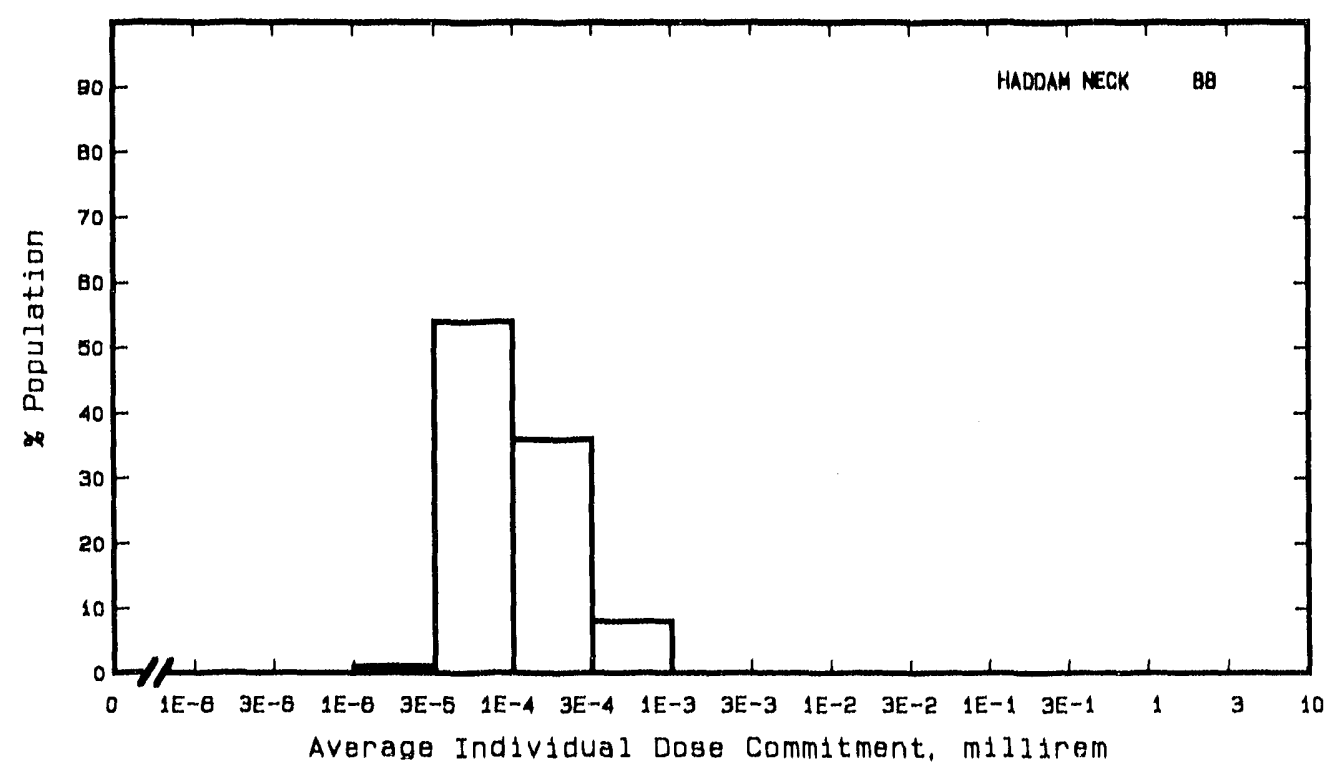


Stte: HARRIS

NEWHILL, NORTH CAROLINA

Location: N $35.6^{\circ} \quad$ W $79.0^{\circ}$

POPULATION DATA

Total Population Within 2-to-80-km Region: 1.4 E6

Major Metropolitan Centers Within Region:

Center

Raleigh-Durham SMSA

Fayetteville

Burlington

Chapel H+11

Sanford population

620,000

61,000

38,000

34,000

16,000

\section{Location}

$32 \mathrm{~km} \mathrm{NE}$

$60 \mathrm{~km} \quad \mathrm{SSE}$

$64 \mathrm{~km} \mathrm{NW}$

$32 \mathrm{~km} \quad \mathrm{NNW}$

$22 \mathrm{~km} \mathrm{SW}$

SITE-SPECIFIC DATA - AIRBORNE PATHWAYS

Average Annual State Production

of Crops and Animal Products

In $80-\mathrm{km}$ Radius Circle

Regional Productivity Factor:

Animal Grazing Factor:

Meteorology Period of Record: 17 JAN 76 - 31 DEC 78 Recovery: $98 \%$
Veg: $2.6 E 7$ kllogram

Mllk: 1.0E8 1iter

Meat: $5.8 E 7$ kilogram

0.9

0.72

SITE-SPECIFIC DATA - WATERBORNE PATHWAYS via CAPE FEAR RIVER

Average River $\mathrm{Flow}$

at Site: $3,200 \mathrm{ft}^{3} / \mathrm{s}$

Drinking Water:

Exposed Population: 180,000

Dilution Factor: 1.18

Fish:

Edible Harvest: $0.024(\mathrm{a}) \mathrm{kg} / \mathrm{yr}$ Dilution Factor: 488 (b)

(a) Average individual consumption rate of $2.4 \mathrm{~kg} / \mathrm{yr}$ given in FES (1974) used in 1 leu of catch data.

(b) Orie percent of population is assumed to obtain fish taken from und luted effluent. 


\section{POPUI.ATION DOSE-COMMITMENT ESTIMATES AND AVERAGE INDIVIDUAL DOSE-COMMITMENT HISTOGRAM FOR \\ HARRIS}

Dose Commitments (person-rem) from Waterborne Pathways Iotal Body

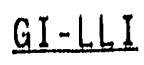

Ihyrotd

Bone

Liver

$\begin{array}{llllll}\text { Infant } & 2.3 \mathrm{E}-02 & 2.3 \mathrm{E}-02 & 2.4 \mathrm{E}-02 & 4.4 \mathrm{E}-05 & 2.3 \mathrm{E}-02 \\ \text { Child } & 3.0 \mathrm{E}-01 & 3.6 \mathrm{E}-01 & 2.9 \mathrm{E}-01 & 4.9 \mathrm{E}-02 & 3.4 \mathrm{E}-01 \\ \text { Teen } & 1.4 \mathrm{E}-01 & 2.9 \mathrm{E}-01 & 1.3 \mathrm{E}-01 & 2.9 \mathrm{E}-02 & 1.7 \mathrm{E}-01 \\ \text { Adu1t } & 1.2 \mathrm{E}+00 & 2.5 \mathrm{E}+00 & 1.1 \mathrm{E}+00 & 1.7 \mathrm{E}-01 & 1.3 \mathrm{E}+00 \\ \text { TOTAL } & 1.7 \mathrm{E}+00 & 3.2 \mathrm{E}+00 & 1.5 \mathrm{E}+00 & 2.4 \mathrm{E}-01 & 1.8 \mathrm{E}+00\end{array}$

Dose Commitments (person-rem) from Atrborne Pathways

Iotal Body GI-LLI Thyrotd Bone Liver Lung

$\begin{array}{lllllll}\text { Infant } & 1.5 \mathrm{E}-03 & 1.5 \mathrm{E}-03 & 1.5 \mathrm{E}-03 & 1.5 \mathrm{E}-03 & 1.5 \mathrm{E}-03 & 1.6 \mathrm{E}-03 \\ \text { Cht1d } & 1.6 \mathrm{E}-02 & 1.6 \mathrm{E}-02 & 1.6 \mathrm{E}-02 & 1.6 \mathrm{E}-02 & 1.6 \mathrm{E}-02 & 1.8 \mathrm{E}-02 \\ \text { Teen } & 1.2 \mathrm{E}-02 & 1.2 \mathrm{E}-02 & 1.2 \mathrm{E}-02 & 1.2 \mathrm{E}-02 & 1.2 \mathrm{E}-02 & 1.4 \mathrm{E}-02 \\ \text { Adu1t } & 7.3 \mathrm{E}-02 & 7.3 \mathrm{E}-02 & 7.3 \mathrm{E}-02 & 7.3 \mathrm{E}-02 & 7.3 \mathrm{E}-02 & 8.0 \mathrm{E}-02 \\ \text { TOTAL } & 1.0 \mathrm{E}-01 & 1.0 \mathrm{E}-\mathrm{O} 1 & 1.0 \mathrm{E}-01 & 1.0 \mathrm{E}-01 & 1.0 \mathrm{E}-01 & 1.1 \mathrm{E}-01\end{array}$

Production/Consumption factors:

Produce: $0.085 \quad$ Milk: $0.51 \quad$ Meat: 0.47

Fraction of Population Fecetving an Average Individual

Total-Body Dose Commitment from Alrborne Pathwaya

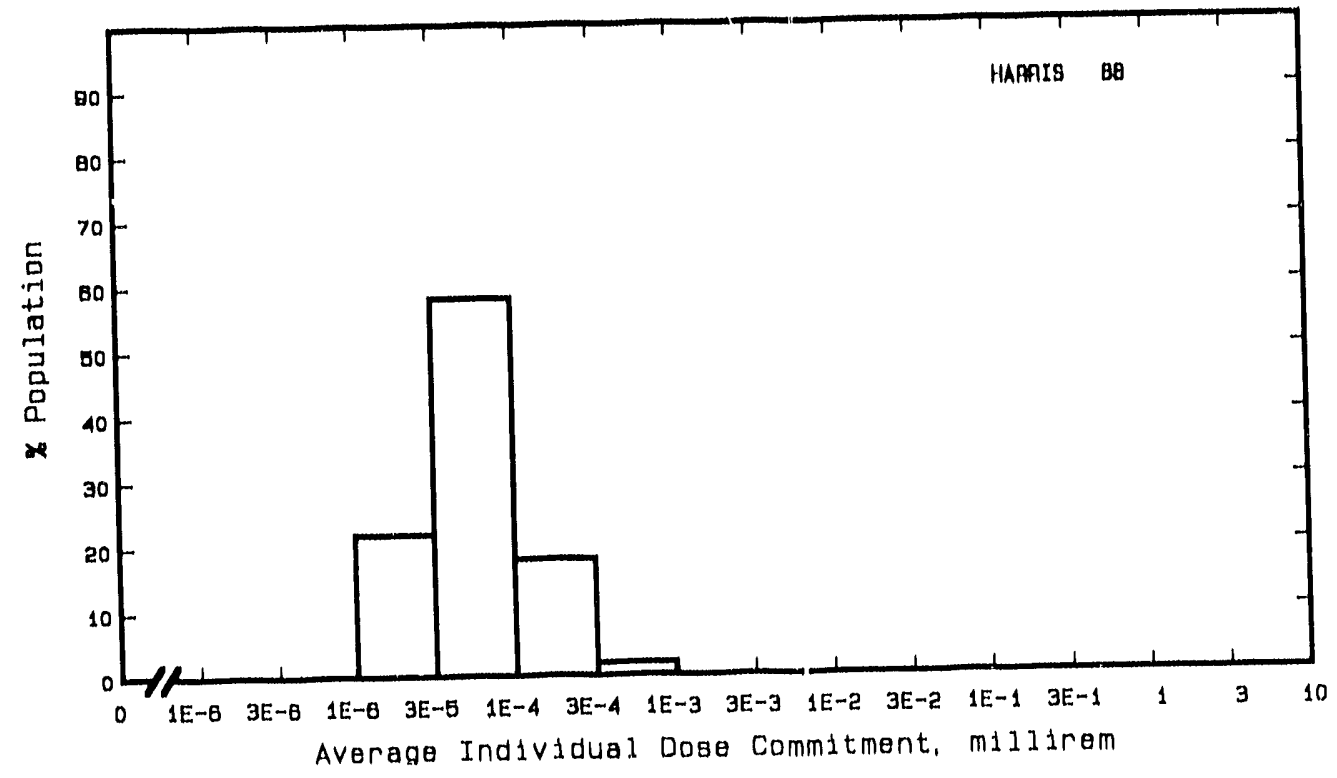


SIte: E. I. HATCH

BAXLEY, GEORGIA

Location: $\quad$ N $31.9342^{\circ} \quad$ W $82.3444^{\circ}$

POPULATION DATA

Total Population Within 2-to-80-k.m Regton: $3.5 E 5$

Major Metropolitan Centers Within Region:

Center

Waycross

Statesboro

Hinesvitle

Douglas

Vidalita
Population

23,000

17,000

13,000

13,000

11,000
Location

$80 \mathrm{~km} \mathrm{~S}$

$78 \mathrm{~km} \quad \mathrm{NE}$

$71 \mathrm{~km} \quad \mathrm{E}$

$67 \mathrm{~km} \quad \mathrm{SW}$

$32 \mathrm{~km} \mathrm{~N}$

SITE-SPECIFIC DATA - AIRBORNE PATHWAYS

Average Annual State Production

of Crops and Animal Products

In $80-\mathrm{km}$ Radius Circle

Regional Productivity Factor:

Antmal Grazing Factor:

Meteorology Period of Record: 1 JUN 70 - 31 AUG 74 Recovery: 87\%
Veg: $8.8 \mathrm{E} 6 \mathrm{ktlogram}$

Milk: 7.0E7 liter

Meat: $8.1 E 7$ kflogram

1

0.8

SITE-SPECIFIC DATA - WATERBORNE PATHWAYS VIa ALTAMAHA RIVER

Average River Flow

at Site: $13,000 \mathrm{ft}^{3} / \mathrm{s}$

Drinking Water:

None

Fish:

Edible Harvest: $6.3 \mathrm{E} 5(\mathrm{a}) \mathrm{kg} / \mathrm{yr}$

Dilution Factor: 1

(a) Commerctal catch plus 3 pounds of game ftsh per year taken from river by average person according to FE.S (1972). 
POPULATION DOSE-COMMITMENT ESTIMATES AND

AVERAGE INDIVIDUAL DOSE-COMMITMENT HISTOGRAM FOR

E. I. HATCH 1 AND 2

Dose Commitments (person-rem) from Waterborne Pathways

Total Body GI-LLI Ihyrold Bone Liver

$\begin{array}{llllll}\text { Infant } & 0.0 E+00 & 0.0 E+00 & 0.0 E+00 & 0.0 E+00 & 0.0 E+00 \\ \text { Chtld } & 2.8 E-01 & 1.6 E-02 & 3.5 E-03 & 1.3 E+00 & 1.5 E+00 \\ \text { Teen } & 5.2 E-01 & 3.2 E-02 & 2.5 E-03 & 7.7 E-01 & 1.3 E+01 \\ \text { Adult } & 5.6 E+00 & 2.8 E-01 & 1.7 E-02 & 4.5 E+00 & 7.7 E+00 \\ \text { TOTAL } & 6.4 E+00 & 3.3 E-01 & 2.3 E-02 & 6.5 E+00 & 1.1 E+01\end{array}$

Dose Commitments (person-rem) from Airborne Pathways

Total Body GI-LLI Ihyroid Bone Liver Lung

$\begin{array}{lllllll}\text { Infant } & 5.0 \mathrm{E}-04 & 5.1 \mathrm{E}-04 & 3.9 \mathrm{E}-03 & 4.9 \mathrm{E}-04 & 6.3 \mathrm{E}-04 & 5.2 \mathrm{E}-04 \\ \text { Child } & 5.9 \mathrm{E}-03 & 5.6 \mathrm{E}-03 & 2.4 \mathrm{E}-02 & 5.2 \mathrm{E}-03 & 6.6 \mathrm{E}-03 & 6.1 \mathrm{E}-03 \\ \text { Teen } & 4.1 \mathrm{E}-03 & 4.0 \mathrm{E}-03 & 1.1 \mathrm{E}-02 & 3.4 \mathrm{E}-03 & 4.4 \mathrm{E}-03 & 4.5 \mathrm{E}-03 \\ \text { Adult } & 2.4 \mathrm{E}-02 & 2.4 \mathrm{E}-02 & 4.7 \mathrm{E}-02 & 2.02 \mathrm{E}-02 & 2.5 \mathrm{E}-02 & 2.5 \mathrm{E}-02 \\ \text { TOTAL } & 3.5 \mathrm{E}-02 & 3.4 \mathrm{E}-02 & 8.6 \mathrm{E}-02 & 2.9 \mathrm{E}-02 & 3.6 \mathrm{E}-02 & 3.6 \mathrm{E}-02\end{array}$

Production/Consumption factors:

Produce: $0.13 \quad$ Mllk: $1.5 \quad$ Meat: 2.9

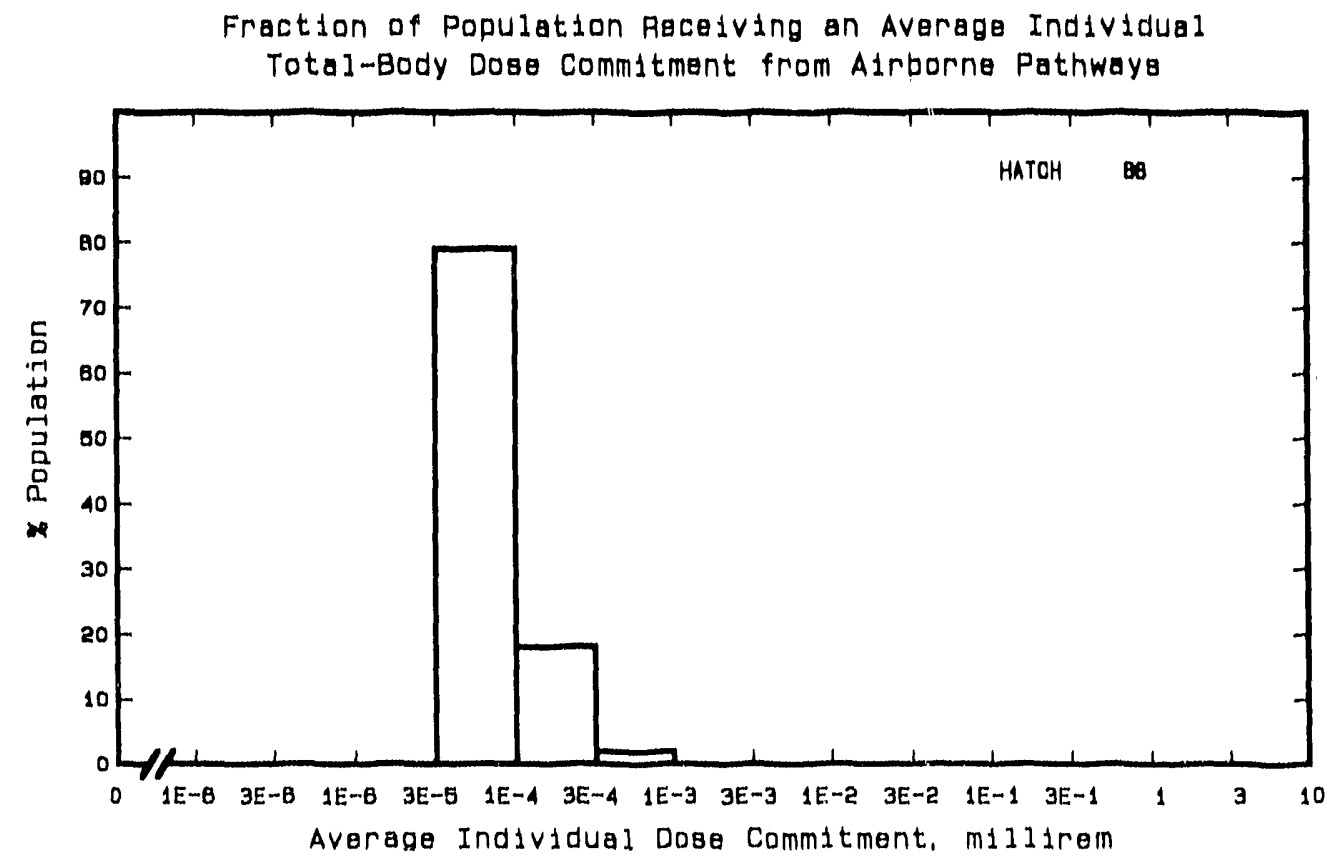


Stte: HOPE CREEK

SALEM COUNTY, NEW JERSEY

Location: $\quad$ N $39.5733^{\circ}$ W $75.4667^{\circ}$

POPULATION DATA

Total Population Within 2-to-80-km Region: $4.9 E 6$

Major Metropolitan Centers Within Region:

Center

Philadelphia

Camden

Wilmington

Vineland

Chester
Population

$$
\begin{array}{r}
1,700,000 \\
88,000 \\
75,000 \\
55,000 \\
46,000
\end{array}
$$

Location

$64 \mathrm{~km} \quad \mathrm{NNE}$

$61 \mathrm{~km} \quad \mathrm{NE}$

$30 \mathrm{~km} \quad \mathrm{~N}$

$38 \mathrm{~km} \quad \mathrm{E}$

$43 \mathrm{~km}$ NNE

SITE-SPECIFIC DATA - AIRBORNE PATHWAYS

Average Annual State Production

of Crops and Animal Products

In $80-\mathrm{km}$ Radius Circle

Regional Productivity Factor:

Animal Grazing Factor:
Veg: $7.4 \mathrm{E} 7 \mathrm{kilogram}$

Milk: 2.7E8 1iter

Meat: $2.4 \mathrm{E} 7 \mathrm{kilogram}$

0.8

0.6

Meteorology Period of Record: 1 JAN 77 - 31 DEC 81 Recovery: $97 \%$

Average Rivt, Flow

at Site: $16,000 \mathrm{ft}^{3} / \mathrm{s}$

Drinking Water:

Fish:

Invertebrates:
None

Edible Harvest: $3.6 E 5 \mathrm{~kg} / \mathrm{yr}$ Dilution Factor: 1

Edible Harvest: $1.6 E 5 \mathrm{~kg} / \mathrm{yr}$

Dilution Factor: 1 
POPULATION DOSE-COMMITMENT ESTIMATES AND AVERAGE INDIVIDUAL DOSE-COMMITMENT HISTOGRAM FOR

HOPE CREEK

Dose Commitments (person-rem) from Waterborne Pathways

Total Body GI-LLI Thyroid Bone Liver

$\begin{array}{llllll}\text { Infant } & 0.0 \mathrm{E}+00 & 0.0 \mathrm{E}+00 & 0.0 \mathrm{E}+00 & 0.0 \mathrm{E}+00 & 0.0 \mathrm{E}+00 \\ \text { Child } & 1.7 \mathrm{E}-01 & 5.5 \mathrm{E}-02 & 7.8 \mathrm{E}-06 & 1.5 \mathrm{E}-01 & 2.8 \mathrm{E}-01 \\ \text { Teen } & 1.1 \mathrm{E}-01 & 1.2 \mathrm{E}-01 & 6.2 \mathrm{E}-06 & 5.4 \mathrm{E}-02 & 2.5 \mathrm{E}-01 \\ \text { Adult } & 6.8 \mathrm{E}-01 & 1.1 \mathrm{E}+00 & 4.5 \mathrm{E}-05 & 6.2 \mathrm{E}-01 & 1.6 \mathrm{E}+00 \\ \text { TOTAL } & 9.6 \mathrm{E}-01 & 1.2 \mathrm{E}+00 & 5.9 \mathrm{E}-05 & 8.5 \mathrm{E}-01 & 2.1 \mathrm{E}+00\end{array}$

Dose Commitments (person-rem) from Airborne Pathways

Total Body GI-LLI Thyroid Bone Liver Lung

$\begin{array}{lllllll}\text { Infant } & 1.5 \mathrm{E}-03 & 1.5 \mathrm{E}-03 & 1.5 \mathrm{E}-03 & 1.2 \mathrm{E}-04 & 1.5 \mathrm{E}-03 & 1.5 \mathrm{E}-03 \\ \text { Child } & 2.3 \mathrm{E}-02 & 2.3 \mathrm{E}-02 & 2.3 \mathrm{E}-02 & 1.4 \mathrm{E}-03 & 2.3 \mathrm{E}-02 & 2.3 \mathrm{E}-02 \\ \text { Teen } & 1.6 \mathrm{E}-02 & 1.6 \mathrm{E}-02 & 1.6 \mathrm{E}-02 & 1.0 \mathrm{E}-03 & 1.6 \mathrm{E}-02 & 1.6 \mathrm{E}-02 \\ \text { Adult } & 9.1 \mathrm{E}-02 & 9.1 \mathrm{E}-02 & 9.1 \mathrm{E}-02 & 6.1 \mathrm{E}-03 & 9.1 \mathrm{E}-02 & 9.1 \mathrm{E}-02 \\ \text { TOTAL } & 1.3 \mathrm{E}-01 & 1.3 \mathrm{E}-01 & 1.3 \mathrm{E}-01 & 8.6 \mathrm{E}-03 & 1.3 \mathrm{E}-01 & 1.3 \mathrm{E}-01\end{array}$

Production/Consumption factors:

Produce: 0.062 Milk: 0.34 Meat: 0.050

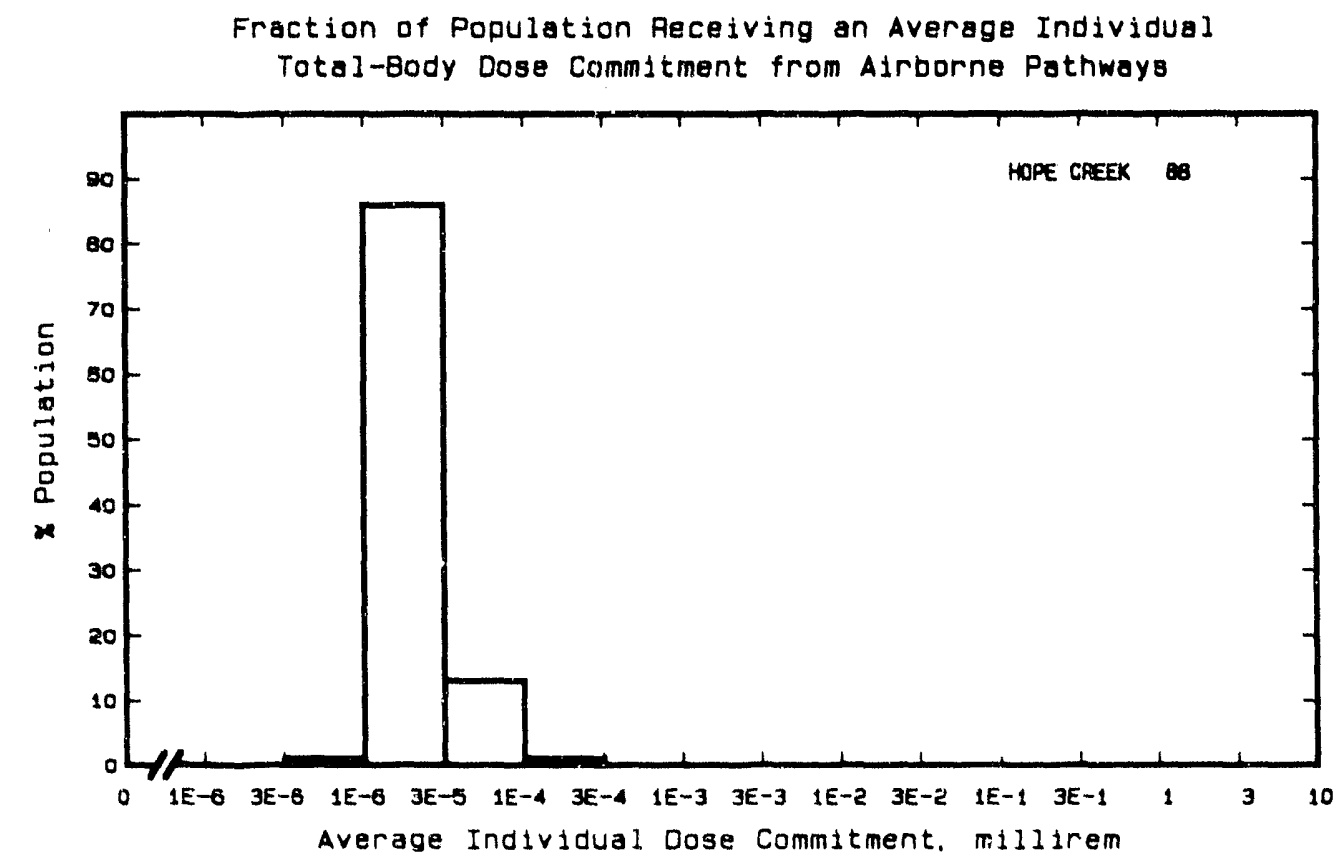

2.57 
Site: INDIAN POINT

BUCHANAN, NEW YORK

Location: $\quad$ N $41.2714^{\circ} \quad$ W $73.9525^{\circ}$

POPULATION DATA

Total Population Within 2-to-80-km Region: 1.6E7

Major Metropolitan Centers Within Region:

Center Population Location

$\begin{array}{lrrl}\text { New York SMSA } & 8,700,000 & 57 \mathrm{~km} & \text { S } \\ \text { Newark SMSA } & 1,900,000 & 62 \mathrm{~km} & \text { SSW } \\ \text { Nassau County SMSA } & 1,300,000 & 70 \mathrm{~km} & \text { SSE } \\ \text { Jersey City SMSA } & 570,000 & 61 \mathrm{~km} & \text { S } \\ \text { Paterson-Clifton-Passaic SMSA } & 450,000 & 44 \mathrm{~km} & \text { SSW }\end{array}$

SITE-SPECIFIC DATA - AIRBORNE PATHWAYS

Average Annual State Production

Veg: $7.6 \mathrm{E} 7 \mathrm{kilogram}$

of Crops and Animal Products

In $80-\mathrm{km}$ Radius Circle

Milk: 7.0E8 liter

Meat: $3.3 E 7$ kilogram

Regional Productivity Factor:

0.8

Animal Grazing Factor:

0.5

Meteorology Period of Record: 1 JAN 75 - 31 DEC 75 Recovery: $96 \%$

SITE-SPECIFIC DATA - WATERBORNE PATHWAYS via HUDSON RIVER

Average River Flow

at Site: $20,000 \mathrm{ft}^{3} / \mathrm{s}$

Drinking Water: None

Fish: Edible Harvest: (a)

Dilution Factor: 0.001 (b)

(a) No fish catch data given in FES (1982), so generic consumption rates used (Table A-1).

(b) One percent of population obtain 10\% of the ir fish from river according to FES (19/2). 
POPULATION DOSE-COMMITMENT ESTIMATES AND

AVERAGE INDIVIDUAL DOSE-COMMITMENT HISTOGRAM FOR

INDIAN POINT 1,2 , AND 3

Dose Commitments (person-rem) from Waterborne Pathways

\begin{tabular}{|c|c|c|c|c|c|}
\hline & Total Body & $\underline{G I-L L I}$ & Thyroid & Bone & Liver \\
\hline $\begin{array}{l}\text { Infant } \\
\text { Child } \\
\text { Teen } \\
\text { Adult }\end{array}$ & $\begin{array}{l}0.0 E+00 \\
4.1 E-03 \\
7.5 E-03 \\
8.0 E-02\end{array}$ & $\begin{array}{l}0.0 E+00 \\
2.7 E-03 \\
5.6 E-03 \\
4.9 E-02\end{array}$ & $\begin{array}{l}0.0 E+00 \\
9.3 E-04 \\
6.8 E-04 \\
4.5 E-03\end{array}$ & $\begin{array}{l}0.0 E+00 \\
2.4 E-02 \\
1.4 E-02 \\
8.3 E-02\end{array}$ & $\begin{array}{l}0.0 E+00 \\
2.2 E-02 \\
1.9 E-02 \\
1.1 E-01\end{array}$ \\
\hline TOTAL & $9.2 E-02$ & $5.7 E-02$ & $6.1 E-03$ & $1.2 E-01$ & $1.5 E-01$ \\
\hline
\end{tabular}

Dose Commitments (person-rem) from Airborne Pathways

Iotal Body GI-LLI Thyroid Bone Liver Lung

$\begin{array}{lllllll}\text { Infant } & 4.8 \mathrm{E}-03 & 4.7 \mathrm{E}-03 & 1.5 \mathrm{E}-02 & 4.9 \mathrm{E}-03 & 5.3 \mathrm{E}-03 & 6.4 \mathrm{E}-03 \\ \text { Child } & 5.5 \mathrm{E}-02 & 5.4 \mathrm{E}-02 & 1.2 \mathrm{E}-01 & 5.3 \mathrm{E}-02 & 5.8 \mathrm{E}-02 & 8.1 \mathrm{E}-02 \\ \text { Teen } & 4.0 \mathrm{E}-01 & 4.0 \mathrm{E}-02 & 7.5 \mathrm{E}-02 & 3.7 \mathrm{E}-02 & 4.2 \mathrm{E}-02 & 6.6 \mathrm{E}-02 \\ \text { Adult } & 2.4 \mathrm{E}-01 & 2.4 \mathrm{E}-01 & 3.9 \mathrm{E}-01 & 2.2 \mathrm{E}-01 & 2.4 \mathrm{E}-01 & 3.4 \mathrm{E}-01 \\ \text { TOTAL } & 3.4 \mathrm{E}-01 & 3.4 \mathrm{E}-01 & 6.0 \mathrm{E}-01 & 3.1 \mathrm{E}-01 & 3.5 \mathrm{E}-01 & 4.9 \mathrm{E}-01\end{array}$

Production/Consumption factors:
Produce: 0.020
Milk: 0.27
Meat: 0.021

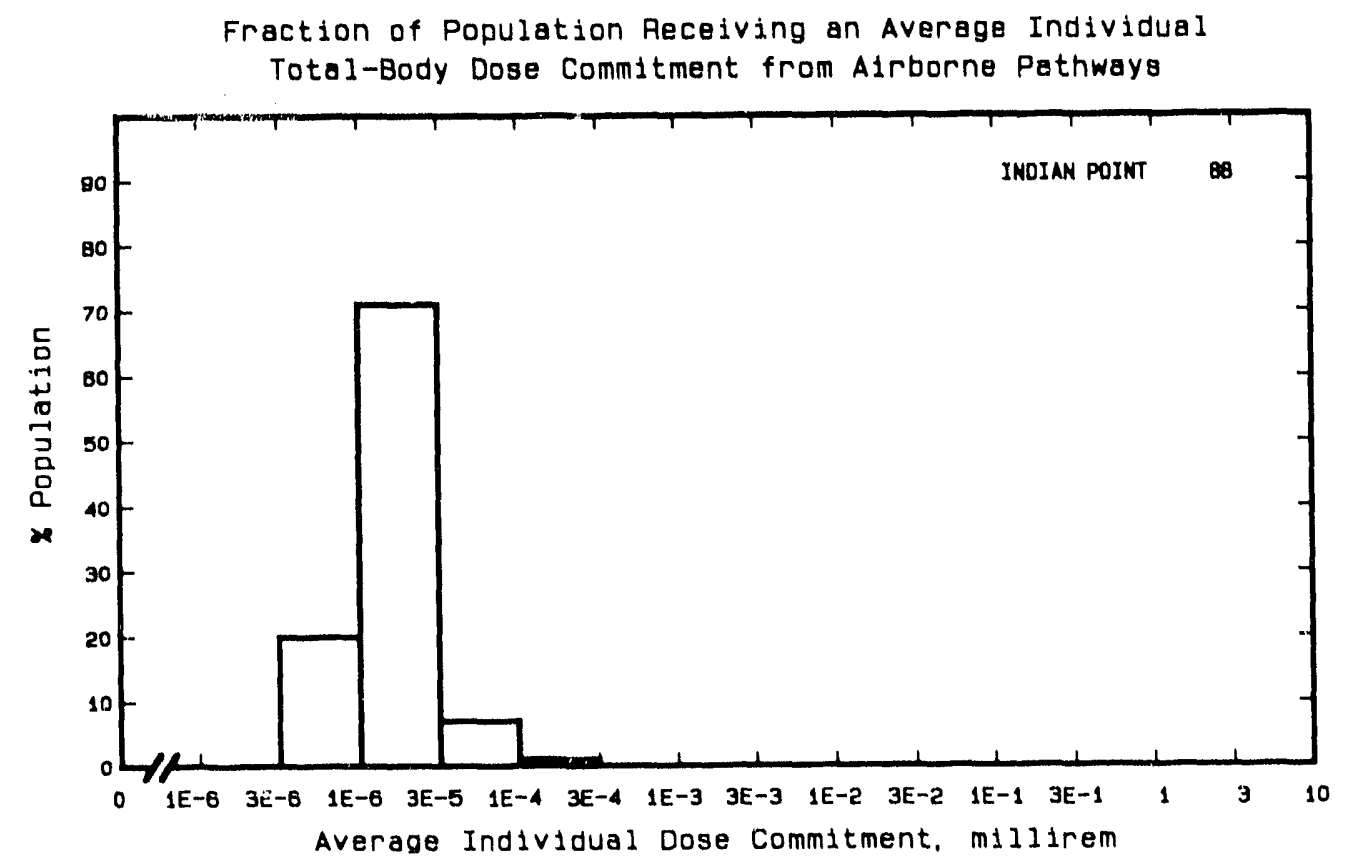


Site: KEWAUNEE

CARLTON, WISCONSIN

Location: $\quad$ N $44.3431^{\circ} \quad$ W $87.5361^{\circ}$

POPULATION DATA

Total Population Within 2-to-80-km Region: 6.3E5

Major Metropolitan Centers Within Region:

\begin{tabular}{lccc} 
Center & Population & \multicolumn{2}{c}{ Location } \\
\cline { 2 - 5 } & & & \\
Greenbay SMSA & 180,000 & $44 \mathrm{~km}$ & NW \\
Appleton SMSA & 290,000 & $72 \mathrm{~km}$ & W \\
Sheboygan & 48,000 & $65 \mathrm{~km}$ & SSW \\
Manitowoc & 33,000 & $29 \mathrm{~km}$ & SSW \\
Neenah & 22,000 & $72 \mathrm{~km}$ & W
\end{tabular}

SITE-SPECIFIC DATA - AIRBORNE PATHWAYS

Average Annual State Production

of Crops and Animal Products

In $80-\mathrm{km}$ Radius Circle

Veg: $7.2 \mathrm{E} 7 \mathrm{kilogram}$

Milk: 1.2E9 liter

Meat: 1.0E8 kilogram

Regional Productivity Factor: $\quad 0.5$

Animal Grazing Factor: $\quad 0.5$

Meteorology Period of Record: 2 JAN 69 - 31 DEC 69 Recovery: $76 \%$

SITE-SPECIFIC DATA - WATERBORNE PATHWAYS via LAKE MICHIGAN

Average Dilution Flow from

Plant: $44 \mathrm{ft}^{3} / \mathrm{s}$

Drinking Water: Exposed Population: 270,000

Dilution Factor: $8.2 \mathrm{E}-3(\mathrm{a})$

Fish:

Edible Harvest: $1.1(\mathrm{~b}) \mathrm{kg} / \mathrm{yr}$
Dilution Factor: $0.01(\mathrm{c})^{\mathrm{g}}$

(a) Drinking water dilution factor estimated by averaging dilution factors derived from FES (1972) suitably weighted for populations.

(b) Average individual consumption rate as given in FES used in lieu of catch data.

(c) Dilution factor reduced $1 / 10$ from that used in FES in consideration of lake mixing. 
POPULATION DOSE-COMNIITMENT ESTIMATES AND

AVERAGE INDIVIDUAL DOSE-COMMITMENT HISTOGRAM FOR

KEWAUNEE

Dose Commitments (person-rem) from Waterborne Pathways

Total Body GI-LLI Thyroid Bone Liver

$\begin{array}{llllll}\text { Infant } & 1.5 \mathrm{E}-02 & 1.5 \mathrm{E}-02 & 1.7 \mathrm{E}-02 & 1.6 \mathrm{E}-03 & 1.6 \mathrm{E}-02 \\ \text { Child } & 1.9 \mathrm{E}-01 & 1.9 \mathrm{E}-01 & 1.8 \mathrm{E}-01 & 1.0 \mathrm{E}-01 & 2.8 \mathrm{E}-01 \\ \text { Teen } & 1.0 \mathrm{E}-01 & 9.7 \mathrm{E}-02 & 6.7 \mathrm{E}-02 & 5.7 \mathrm{E}-02 & 1.6 \mathrm{E}-01 \\ \text { Adult } & 9.5 \mathrm{E}-01 & 8.4 \mathrm{E}-01 & 5.6 \mathrm{E}-01 & 3.3 \mathrm{E}-01 & 1.1 \mathrm{E}+00 \\ \text { TOTAL } & 1.3 \mathrm{E}+00 & 1.1 \mathrm{E}+00 & 8.2 \mathrm{E}-01 & 5.0 \mathrm{E}-01 & 1.5 \mathrm{E}+00\end{array}$

Dose Commitments (person-rem) from Airborne Pathways

\begin{tabular}{lcccccc} 
& Total Body & GI-LLI & Thyroid & Bone & Liver & Lung \\
\cline { 2 - 4 } Infant & $2.9 \mathrm{E}-05$ & $2.8 \mathrm{E}-05$ & $7.8 \mathrm{E}-05$ & $2.5 \mathrm{E}-06$ & $2.9 \mathrm{E}-05$ & $2.9 \mathrm{E}-05$ \\
Child & $4.5 \mathrm{E}-04$ & $4.4 \mathrm{E}-04$ & $8.0 \mathrm{E}-04$ & $6.1 \mathrm{E}-05$ & $4.4 \mathrm{E}-04$ & $4.5 \mathrm{E}-04$ \\
Teen & $2.7 \mathrm{E}-04$ & $2.7 \mathrm{E}-04$ & $4.3 \mathrm{E}-04$ & $3.6 \mathrm{E}-05$ & $2.7 \mathrm{E}-04$ & $2.7 \mathrm{E}-04$ \\
Adult & $1.5 \mathrm{E}-03$ & $1.5 \mathrm{E}-03$ & $2.1 \mathrm{E}-03$ & $1.9 \mathrm{E}-04$ & $1.5 \mathrm{E}-03$ & $1.5 \mathrm{E}-03$ \\
TOTAL & $2.2 \mathrm{E}-03$ & $2.2 \mathrm{E}-03$ & $3.4 \mathrm{E}-03$ & $2.9 \mathrm{E}-04$ & $2.2 \mathrm{E}-03$ & $2.2 \mathrm{E}-03$
\end{tabular}

Production/Consumption factors:
Produce: 0.30
Milk: 7.1
Meat: 1.0

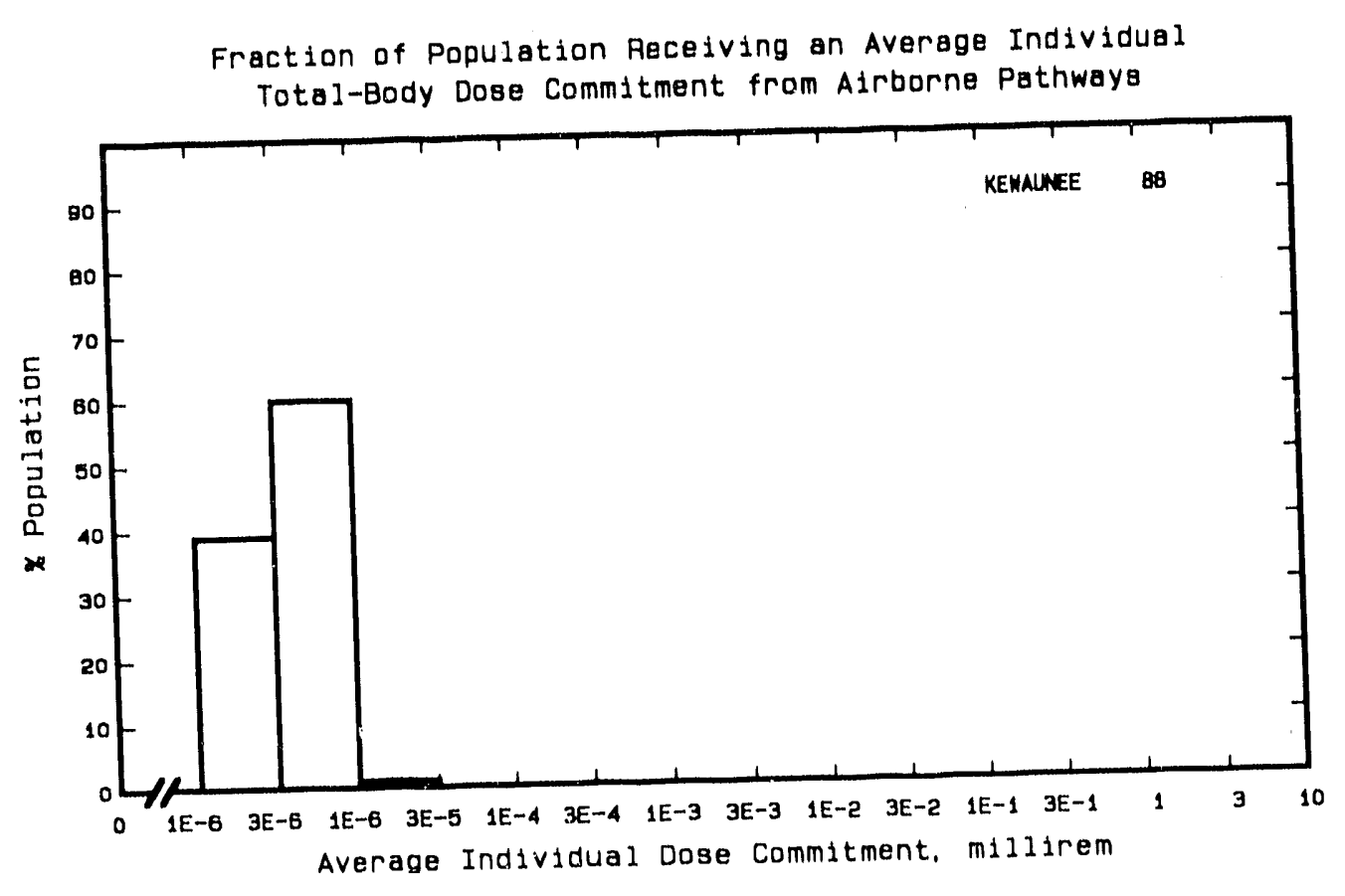


Site: LACROSSE

GENOA, WISCONSIN

Location: $\quad$ N $43.5583^{\circ} \quad$ W $91.2306^{\circ}$

POPULATION DATA

Total Population Within 2-to-80-km Region: 3.5E5

\begin{tabular}{|c|c|c|}
\hline Center & Population & Location \\
\hline $\begin{array}{l}\text { La Crosse SMSA } \\
\text { Winona }\end{array}$ & $\begin{array}{l}94,000 \\
25,000\end{array}$ & $\begin{array}{l}27 \mathrm{~km} \\
64 \mathrm{~km}\end{array}$ \\
\hline
\end{tabular}

SITE-SPECIFIC DATA - AIRBORNE PATHWAYS

Average Annual State Production

Veg: $7.2 E 7$ kilogram

of Crops and Animal Products

Mi1k: 1.2E9 1iter

In $80-\mathrm{km}$ Radius Circle

Meat: 1.0 E8 kilogram

Regional Productivity Factor:

Animal Grazing Factor:

1

0.5

Meteorology Period of Record: 1 JAN 75 - 31 DEC 75 Recovery: $97 \%$

SITE-SPECIFIC DATA - WATERBORNE PATHWAYS via MISSISSIPPI RIVER

Average River

Flow at Site: $28,000 \mathrm{ft}^{3} / \mathrm{s}$

Drinking Water: None

Fish: Edible Harvest: (a)

Dilution Factor: $0.5^{(a)}$

(a) No fish catch data given in FES (1976), so $1 / 2$ population assumed to consume river fish at generic consumption rates (Table $A-1$ ). 
POPULATION DOSE-COMMITMENT ESTIMATES AND

AVERAGE INDIVIDUAL. DOSE-COMMITMENT HISTOGRAM FOR

\section{LACROSSE}

Dose Commitments (person-rem) from Waterborne Pathways

Total Body GI-LLI Thyroid Bone Liver

$\begin{array}{llllll}\text { Infant } & 0.0 \mathrm{E}+00 & 0.0 \mathrm{E}+00 & 0.0 \mathrm{E}-400 & 0.0 \mathrm{E}+00 & 0.0 \mathrm{E}+00 \\ \text { Child } & 1.7 \mathrm{E}-02 & 1.9 \mathrm{E}-03 & 2.1 \mathrm{E}-06 & 1.2 \mathrm{E}-01 & 1.1 \mathrm{E}-01 \\ \text { Teen } & 3.3 \mathrm{E}-02 & 3.9 \mathrm{E}-03 & 1.9 \mathrm{E}-06 & 6.9 \mathrm{E}-02 & 9.1 \mathrm{E}-02 \\ \text { Adult } & 3.6 \mathrm{E}-01 & 3.4 \mathrm{E}-02 & 1.5 \mathrm{E}-05 & 4.0 \mathrm{E}-01 & 5.3 \mathrm{E}-01 \\ \text { TOTAL } & 4.1 \mathrm{E}-01 & 4.0 \mathrm{E}-02 & 1.9 \mathrm{E}-05 & 5.8 \mathrm{E}-01 & 7.3 \mathrm{E}-01\end{array}$

Dose Commitments (person-rem) from Airborne Pathways

Total Body GI-LLI Thyroid Bone Liver Lung

$\begin{array}{lllllll}\text { Infant } & 2.4 \mathrm{E}-06 & 2.4 \mathrm{E}-06 & 2.4 \mathrm{E}-06 & 9.9 \mathrm{E}-08 & 2.5 \mathrm{E}-06 & 2.5 \mathrm{E}-06 \\ \text { Child } & 5.7 \mathrm{E}-05 & 5.7 \mathrm{E}-05 & 5.7 \mathrm{E}-05 & 1.6 \mathrm{E}-06 & 5.8 \mathrm{E}-05 & 5.8 \mathrm{E}-05 \\ \text { Teen } & 3.4 \mathrm{E}-05 & 3.4 \mathrm{E}-05 & 3.4 \mathrm{E}-05 & 5.9 \mathrm{E}-07 & 3.4 \mathrm{E}-05 & 3.4 \mathrm{E}-05 \\ \text { Adu1t } & 1.8 \mathrm{E}-04 & 1.8 \mathrm{E}-04 & 1.8 \mathrm{E}-04 & 2.4 \mathrm{E}-06 & 1.8 \mathrm{E}-04 & 1.8 \mathrm{E}-04 \\ \text { TOTAL } & 2.8 \mathrm{E}-04 & 2.8 \mathrm{E}-04 & 2.8 \mathrm{E}-04 & 4.7 \mathrm{E}-06 & 2.8 \mathrm{E}-04 & 2.8 \mathrm{E}-04\end{array}$

Production/Consumption factors:

Produce: $1.0 \quad$ Milk: $25 \quad$ Meat: 3.6

Fraction of Population Receiving an Average Individual

Total-Body Dose Commitment from Alrborne Pathwaya

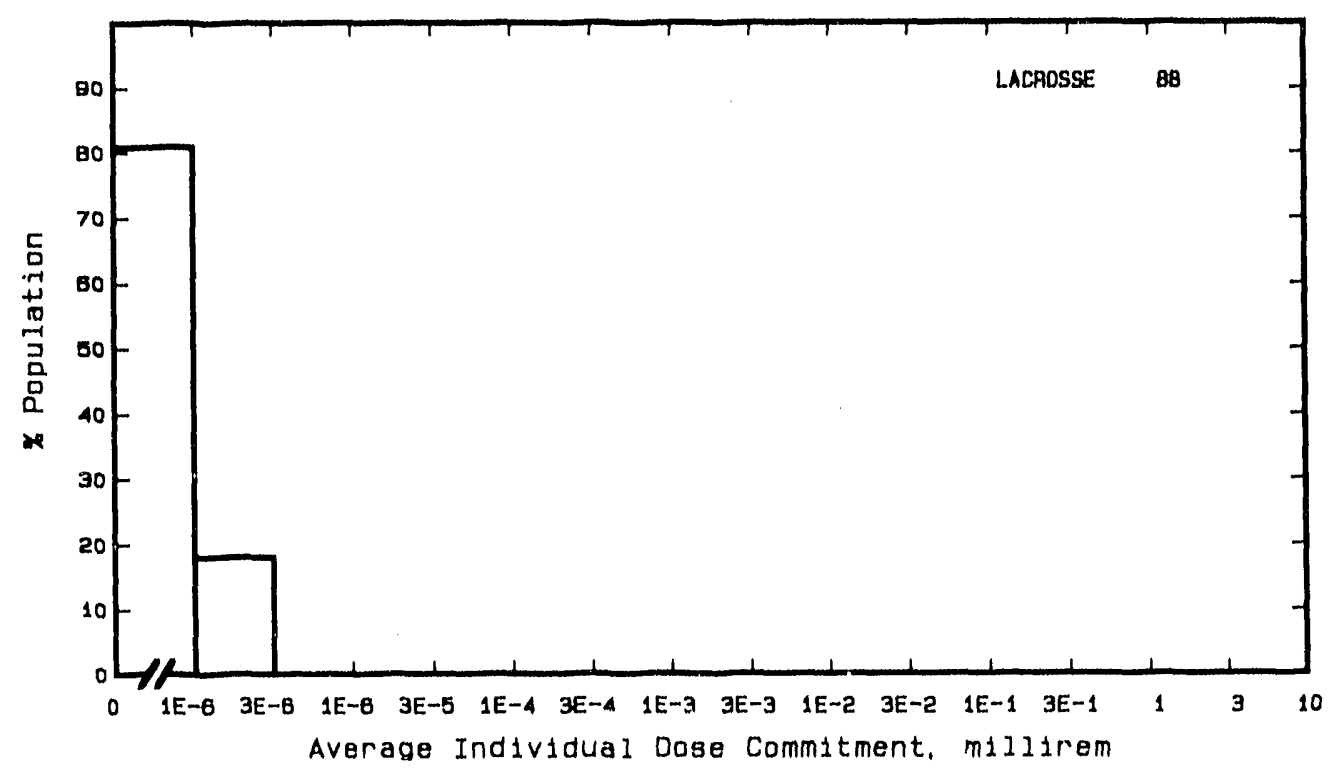


Site: LASALLE

SENECA, ILLINOIS

Location: $\quad$ N $41.2439^{\circ} \quad W 88.6708^{\circ}$

POPULATION DATA

Total Population Within 2-to-80-km Region: 1.1E6

Major Metropolitan Centers Within Region:

Center

Joliet

Aurora

Kankakee

Dekalb

Naperville
Population

78,000

80,000

100,000

33,000

42,000
Location

$59 \mathrm{~km} \quad \mathrm{NNE}$

$65 \mathrm{~km} \quad \mathrm{NNE}$

$69 \mathrm{~km} \quad \mathrm{ESE}$

$77 \mathrm{~km} \quad \mathrm{~N}$

$73 \mathrm{~km} \quad \mathrm{NE}$

SITE-SPECIFIC DATA - AIRBORNE PATHWAYS

Average Annual State Production

of Crops and Animal Products

In $80-\mathrm{km}$ Radius Circle

Regional Productivity Factor:

Animal Grazing Factor:

Meteorology Period of Record: 1 Jan 82 - 31 Dec 82 Recovery: $99 \%$
Veg: 1.1 E8 kilogram

Milk: 1.8E8 liter

Meat: 1.9 E8 kilogram

0.9

0.5

SITE-SPECIFIC DATA - WATERBORNE PATHWAYS VIa ILLINOIS RIVER

Average River Flow

at site: $11,000 \mathrm{ft}^{3} / \mathrm{s}$

Drinking Water: None

Fish:

None $(a)$

(a) Because of river quality, no fish consumption is considered according to FES (1973). 


\section{POPULATION DOSE-COMMITMENT ESTIMATES AND \\ AVERAGE INDIVIDUAL DOSE-COMMITMENT HISTOGRAM FOR}

LASALLE 1 AND 2

Dose Commitments (person-rem) from Waterborne Pathways

Total Body GI-LII Ihyroid Bone Liver

Infant

Child

Teen

Adult

(Little or No Waterborne Pathway Doses)

TOTAL

Dose Commitments (person-rem) from Airborne Pathways

Total Body GI-LII Thyroid Bone Liver Lung

$\begin{array}{lllllll}\text { Infant } & 3.9 \mathrm{E}-03 & 3.9 \mathrm{E}-03 & 1.1 \mathrm{E}-02 & 3.9 \mathrm{E}-03 & 3.9 \mathrm{E}-03 & 4.0 \mathrm{E}-03 \\ \text { Child } & 4.4 \mathrm{E}-02 & 4.4 \mathrm{E}-02 & 1.0 \mathrm{E}-01 & 4.4 \mathrm{E}-02 & 4.4 \mathrm{E}-02 & 4.5 \mathrm{E}-02 \\ \text { Teen } & 3.2 \mathrm{E}-02 & 3.2 \mathrm{E}-02 & 5.7 \mathrm{E}-02 & 3.2 \mathrm{E}-02 & 3.2 \mathrm{E}-02 & 3.4 \mathrm{E}-02 \\ \text { Adult } & 1.9 \mathrm{E}-01 & 1.9 \mathrm{E}-01 & 2.9 \mathrm{E}-01 & 1.9 \mathrm{E}-01 & 1.9 \mathrm{E}-01 & 2.0 \mathrm{E}-01 \\ \text { TOTAL } & 2.7 \mathrm{E}-01 & 2.7 \mathrm{E}-01 & 4.6 \mathrm{E}-01 & 2.7 \mathrm{E}-01 & 2.7 \mathrm{E}-01 & 2.8 \mathrm{E}-01\end{array}$

Production/Consumption factors:

Produce: 0.49 Milk: 1.1 Meat: 2.0

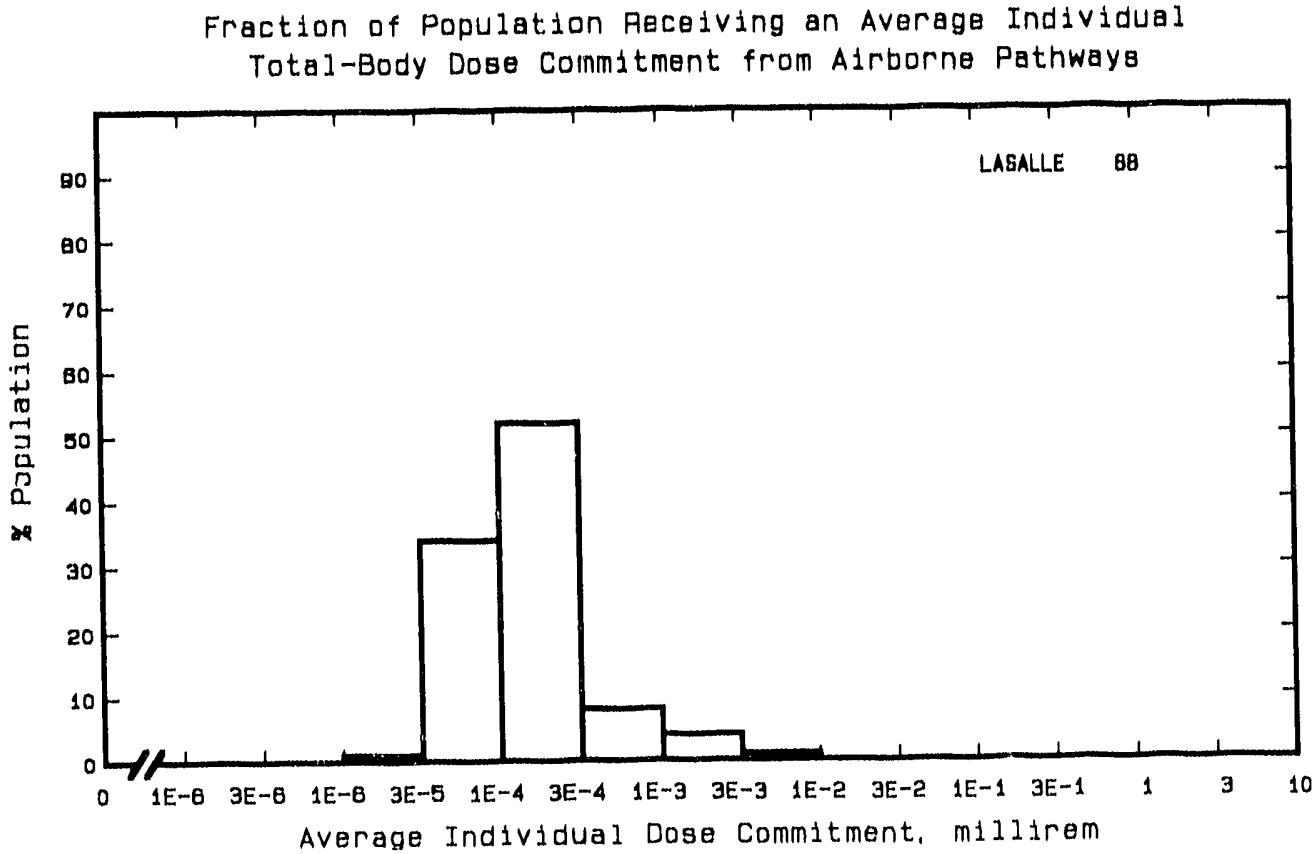


Site: LIMERICK

POTTSTOWN, PENNSYLVANIA

Location: $\quad$ N $40.2242^{\circ} \quad W 75.5875^{\circ}$

POPULATION DATA

Total Population Within 2-to-80-km Region: 6.9E6

Major Metropolitan Centers Within Region:

Center

Population

Location

Philadelphia PMSA

All entown-Bethlehem MSA

$4,800,000$

$40 \mathrm{~km}$ ESE

Reading MSA

66,000

$42 \mathrm{~km} \quad \mathrm{ENE}$

Lancaster MSA

310,000

Wilmington PMSA

360,000

$34 \mathrm{~km} \quad W N W$

Camdon

550,000

$68 \mathrm{~km} \quad W S W$

Trenton PMSA

110,000

$56 \mathrm{~km} \mathrm{~S}$

$52 \mathrm{~km} \quad \mathrm{SE}$

310,000

SITE-SPECIFIC DATA - AIRBORNE PATHWAYS

Average Annual State Production

of Crops and Animal Products

In $80-\mathrm{km}$ Radius Circle

Regional Productivity Factor:

Animal Grazing Factor:
Veg: $5.3 \mathrm{E} 7$ kilogram

Milk: 5.3E8 liter

Meat: $5.4 E 7$ kilogram

0.9

0.6

Meteorology Pertod of Record: 1 Jan 74 - 31 Dec 74 Recovery: $93 \%$

Drinking Water: None

Fish:

None 


\section{POPULATION DOSE-COMMITMENT ESTIMATES AND \\ AVERAGE INDIVIDUAL. DOSE-COMMITMENT HISTOGRAM FOR \\ LIMERICK}

Dose Commltments (person-rem) from Waterborne Pathways

Total Body GI-LII Ihyroid Bone Liver

Infant

Child

Teen

Adult

TOTAL

(No Waterborne Pathway Doses) (a)

Dose Commitments (person-rem) from Airborne Pathways

Total Body GI-LII Thyrotd Bone Liver Lung

$\begin{array}{lllllll}\text { Infant } & 3.1 \mathrm{E}-03 & 3.1 \mathrm{E}-03 & 1.8 \mathrm{E}-02 & 1.1 \mathrm{E}-03 & 3.1 \mathrm{E}-03 & 3.1 \mathrm{E}-03 \\ \text { Chi1d } & 4.2 \mathrm{E}-02 & 4.2 \mathrm{E}-02 & 1.3 \mathrm{E}-01 & 1.2 \mathrm{E}-02 & 4.2 \mathrm{E}-02 & 4.2 \mathrm{E}-02 \\ \text { Teen } & 3.1 \mathrm{E}-02 & 3.1 \mathrm{E}-02 & 7.2 \mathrm{E}-02 & 8.7 \mathrm{E}-03 & 3.1 \mathrm{E}-02 & 3.2 \mathrm{E}-02 \\ \text { Adu7t } & 1.8 \mathrm{E}-01 & 1.8 \mathrm{E}-01 & 3.4 \mathrm{E}-01 & 5.2 \mathrm{E}-02 & 1.8 \mathrm{E}-01 & 1.8 \mathrm{E}-01 \\ \text { TOTAL. } & 2.5 \mathrm{E}-01 & 2.5 \mathrm{E}-01 & 5.6 \mathrm{E}-01 & 7.4 \mathrm{E}-02 & 2.5 \mathrm{E}-01 & 2.6 \mathrm{E}-01\end{array}$

Production/Consumption factors:

Produce: $0.036 \quad$ Milk: 0.53 Meat: 0.089

Fraction of Population Feceiving an Average Individual

Total-Body Dose Commitment from Adrborne Pathways

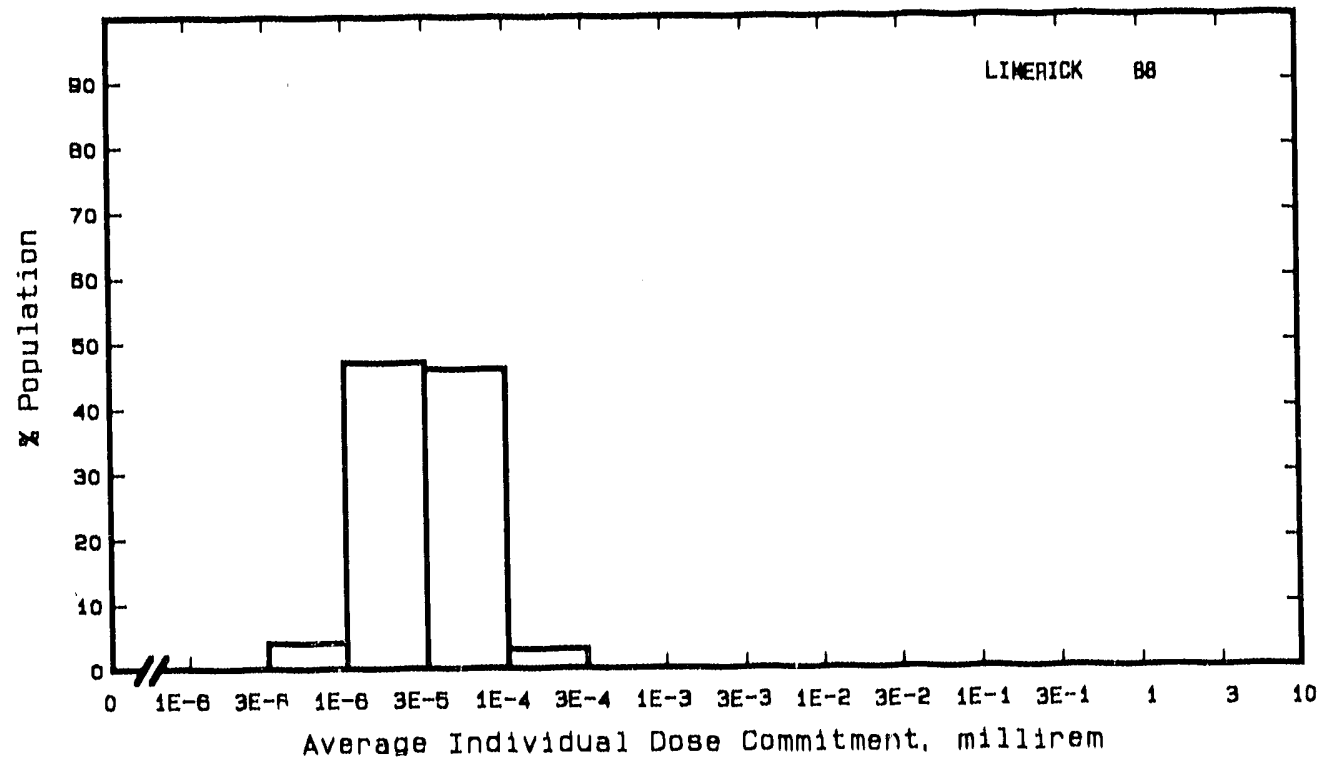

(a) No liquid releases reported (Ttchler, et al., 1991). 
Stte: MAINE YANKEE

Location: $\quad$ N $43.9506^{\circ} \quad$ W $69.6961^{\circ}$

POPULATION DATA

Total Population Within 2-to-80-km Region: $6.3 \mathrm{E} 5$

Major Metropolitan Centers Within Region:

\begin{tabular}{lcccc} 
Center & Population & \multicolumn{2}{c}{ Location } \\
\cline { 2 - 5 } Portiand SMSA & 180,000 & & $56 \mathrm{~km}$ & WSW \\
Lewiston-Auburn SMSA & 76,000 & $45 \mathrm{~km}$ & WNW \\
Augusta & 22,000 & $41 \mathrm{~km}$ & $\mathrm{~N}$ \\
Biddeford & 20,000 & $80 \mathrm{~km}$ & SW \\
Waterville & 18,000 & $67 \mathrm{~km}$ & $\mathrm{~N}$
\end{tabular}

SITE-SPECIFIC DATA - AIRBORNE PATHWAYS

Average Annual State Production

of Crops and Animal Products

In $80-\mathrm{km}$ Radius Circle

Regional Productivity Factor:

Animal Grazing Factor:
Veg: 2.4E8 kilogram

Milk: 6.6E7 1iter

Meat: 4.3E6 kilogram

0.6

0.5

Meteorology Period of Record: 1 APR 75 - 31 MAR 76 Recovery: 98\%

Average Dilution Flow

from Plant: $760 \mathrm{ft}^{3} / \mathrm{s}$

Fish:

Invertebrates:
Edible Harvest: (a)

Dilution Factor: 0.001

Edible Harvest: (a)

Dilution Factor: 0.002

(a) No seafood harvest data given in FES (1972), thus generic population consumption rates used (Table A-1). 
POPULATION DOSE-COMMITMENT ESTIMATES AND

AVERAGE INDIVIDUAL DOSE-COMMITMENT HISTOGRAM FOR

MAINE YANKEE

Dose Commitments (person-rem) from Waterborne Pathways

Iotal Body GI-LLI Thyrotd Bone Liver

$\begin{array}{llllll}\text { Infant } & 0.0 \mathrm{E}+00 & 0.0 \mathrm{E}+00 & 0.0 \mathrm{E}+00 & 0.0 \mathrm{E}+00 & 0.0 \mathrm{E}+00 \\ \text { Child } & 4.5 \mathrm{E}-04 & 9.8 \mathrm{E}-04 & 7.9 \mathrm{E}-05 & 1.7 \mathrm{E}-03 & 1.0 \mathrm{E}-03 \\ \text { Teen } & 3.1 \mathrm{E}-04 & 2.0 \mathrm{E}-03 & 6.0 \mathrm{E}-05 & 9.4 \mathrm{E}-04 & 7.9 \mathrm{E}-04 \\ \text { Adult } & 2.1 \mathrm{E}-03 & 1.7 \mathrm{E}-02 & 4.2 \mathrm{E}-04 & 5.5 \mathrm{E}-03 & 4.6 \mathrm{E}-03 \\ \text { TOTAL. } & 2.9 \mathrm{E}-03 & 2.0 \mathrm{E}-02 & 5.6 \mathrm{E}-04 & 8.1 \mathrm{E}-03 & 5.5 \mathrm{E}-03\end{array}$

Dose Commitments (person-rem) from Airborne Pathways

Iotal Body GI-LLI Ihyrotd Bone Liver Lung

$\begin{array}{lllllll}\text { Infant } & 4.4 \mathrm{E}-05 & 4.4 \mathrm{E}-05 & 1.2 \mathrm{E}-04 & 2.2 \mathrm{E}-05 & 4.4 \mathrm{E}-05 & 4.6 \mathrm{E}-05 \\ \text { Child } & 9.4 \mathrm{E}-04 & 9.4 \mathrm{E}-04 & 2.1 \mathrm{E}-03 & 2.5 \mathrm{E}-04 & 9.4 \mathrm{E}-04 & 9.7 \mathrm{E}-04 \\ \text { Teen } & 5.8 \mathrm{E}-04 & 5.8 \mathrm{E}-04 & 1.1 \mathrm{E}-03 & 1.8 \mathrm{E}-04 & 5.8 \mathrm{E}-04 & 6.2 \mathrm{E}-04 \\ \text { Adult } & 3.2 \mathrm{E}-03 & 3.2 \mathrm{E}-03 & 5.1 \mathrm{E}-03 & 1.1 \mathrm{E}-03 & 3.2 \mathrm{E}-03 & 3.3 \mathrm{E}-03 \\ \text { TOTAL } & 4.7 \mathrm{E}-03 & 4.7 \mathrm{E}-03 & 8.5 \mathrm{E}-03 & 1.5 \mathrm{E}-03 & 4.7 \mathrm{E}-03 & 4.9 \mathrm{E}-03\end{array}$

Production/Consumption factors:

Produce: $1.2 \quad$ Milk: $0.48 \quad$ Meat: 0.051

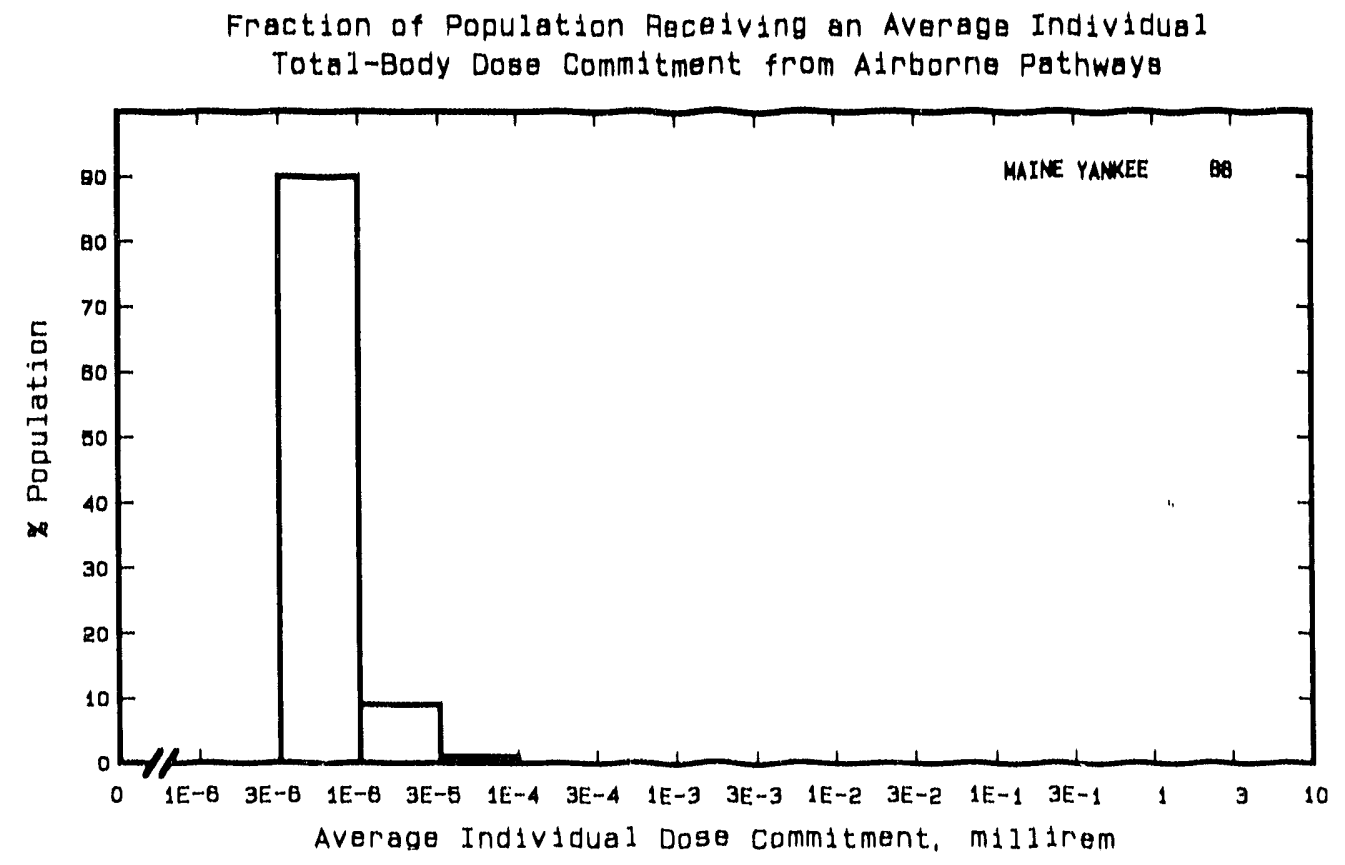


Stte: McGUIRE

Location: $\quad N 35.4322^{\circ} \quad W 80.9483^{\circ}$

POPULATION DATA

Total Population Within 2-to-80-km Region: $1.8 \mathrm{E} 6$

Major Metropolitan Centers Within Region:

\begin{tabular}{lccc}
\multicolumn{1}{c}{ Center } & Population & \multicolumn{2}{c}{ Location } \\
Charlotte-Gastonta SMSA & 700,000 & & \\
Rockhil1 & 37,000 & $25 \mathrm{~km}$ & $\mathrm{~S}$ \\
Kannapolits & 37,000 & $57 \mathrm{~km}$ & $\mathrm{~S}$ \\
Salisbury & 25,000 & $30 \mathrm{~km}$ & $\mathrm{E}$ \\
Hickory & 23,000 & $51 \mathrm{~km}$ & ENE \\
& & $49 \mathrm{~km}$ & $\mathrm{NW}$
\end{tabular}

\section{SITE-SPECIFIC DATA - AIRBORNE PATHWAYS}

Average Annual State Production

of Crops and Antmal Products

In $80-\mathrm{km}$ Radius Circle

Veg: $2.6 \mathrm{E} 7 \mathrm{kilogram}$

Milk: 1.0E8 1iter

Regional Productivity Factor:

Meat: $5.8 \mathrm{E} 7$ kilogram

Animal Grazing Factor:

0.9

0.7

Meteorology Pertod of Record: 17 OCT 70 - 16 OCT 71 Recovery: $90 \%$

SITE-SPECIFIC DATA - WATERBORNE PATHWAYS v Ia LAKE NORMAN On CATAWBA

RIVER

$\begin{array}{ll} & \text { Average River } \mathrm{Flow} \\ & \text { at Site: } 2,600 \mathrm{ft}^{3} / \mathrm{s} \\ \text { Drinking Water: } & \text { Exposed Population: } 770,000 \\ \text { Fish: } & \text { Edible Harvest: } 2.0 \mathrm{E} 5(\mathrm{a}) \mathrm{kg} / \mathrm{yr} \\ & \text { Dilution Factor: } 1\end{array}$

(a) Edible harvest from downstream reservoirs derived from creel surveys obtained from James $R$. Thornton, Duke Power Company. 
POPULATION DOSE-COMMITMENT ESTIMATES AND

AVERAGE INDIVIDUAL DOSE-COMMITMENT HISTOGRAM FOR

MCGUIRE 1 AND 2

Dose Commitments (person-rem) from Waterborne Pathways

Total Body GI-LLI Thyroid Bone Liver

$\begin{array}{llllll}\text { Infant } & 3.0 \mathrm{E}-01 & 3.1 \mathrm{E}-01 & 1.6 \mathrm{E}+00 & 3.1 \mathrm{E}-02 & 3.1 \mathrm{E}-01 \\ \text { Child } & 3.4 \mathrm{E}+00 & 4.1 \mathrm{E}+00 & 1.2 \mathrm{E}+01 & 4.8 \mathrm{E}-01 & 3.5 \mathrm{E}+00 \\ \text { Teen } & 1.3 \mathrm{E}+00 & 2.4 \mathrm{E}+00 & 4.0 \mathrm{E}+00 & 1.6 \mathrm{E}-01 & 1.4 \mathrm{E}+00 \\ \text { Adult } & 1.1 \mathrm{E}+01 & 2.2 \mathrm{E}+01 & 3.0 \mathrm{E}+01 & 9.8 \mathrm{E}-01 & 1.1 \mathrm{E}+01 \\ \text { TOTAL } & 1.6 \mathrm{E}+01 & 2.8 \mathrm{E}+01 & 4.8 \mathrm{E}+01 & 1.7 \mathrm{E}+00 & 1.7 \mathrm{E}+01\end{array}$

Dose Commitments (person-rem) from Airborne Pathways

Total Body GI-LLI Thyroid Bone Liver Lung

$\begin{array}{lllllll}\text { Infant } & 2.4 \mathrm{E}-03 & 2.4 \mathrm{E}-03 & 1.1 \mathrm{E}-02 & 2.2 \mathrm{E}-03 & 2.4 \mathrm{E}-03 & 2.6 \mathrm{E}-03 \\ \text { Child } & 2.8 \mathrm{E}-02 & 2.8 \mathrm{E}-02 & 7.9 \mathrm{E}-02 & 2.4 \mathrm{E}-02 & 2.8 \mathrm{E}-02 & 3.0 \mathrm{E}-02 \\ \text { Teen } & 2.0 \mathrm{E}-02 & 2.0 \mathrm{E}-02 & 4.1 \mathrm{E}-02 & 1.7 \mathrm{E}-02 & 2.0 \mathrm{E}-02 & 2.3 \mathrm{E}-02 \\ \text { Adult } & 1.2 \mathrm{E}-01 & 1.2 \mathrm{E}-01 & 2.0 \mathrm{E}-01 & 1.1 \mathrm{E}-01 & 1.2 \mathrm{E}-01 & 1.3 \mathrm{E}-01 \\ \text { TOTAL } & 1.7 \mathrm{E}-01 & 1.7 \mathrm{E}-01 & 3.3 \mathrm{E}-01 & 1.5 \mathrm{E}-01 & 1.7 \mathrm{E}-01 & 1.9 \mathrm{E}-01\end{array}$

Production/Consumption factors:

Produce: 0.067 Milk: $0.40 \quad$ Meat: 0.37

Fraction of Population Receiving an Average Individual

Total-Body Dose Commitment from Alrborne Pathways

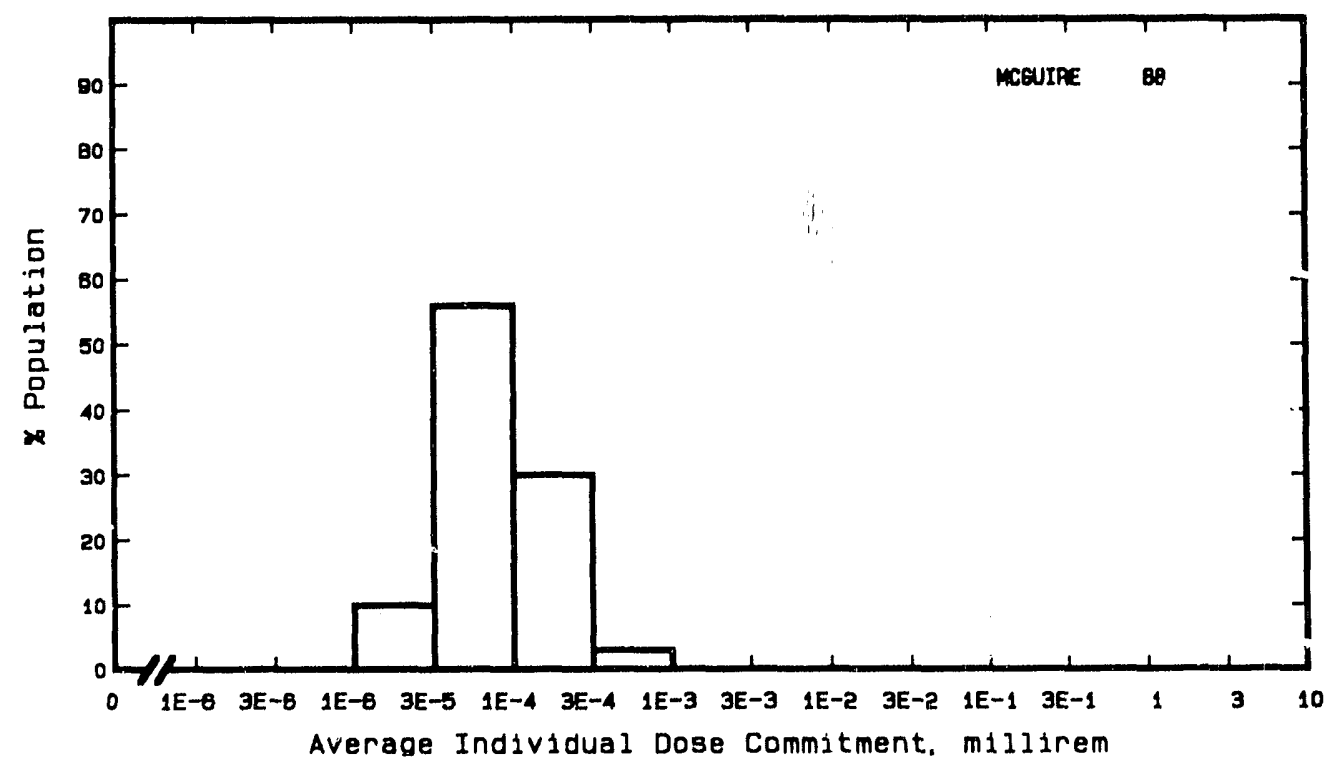


Site: MILLSTONE

WATERFORD, CONNECTICUT

Location: $\quad$ N $41.3086^{\circ} \quad$ W $72.1681^{\circ}$

POPULATION DATA

Total Population Within 2-to-80-km Region: 2.6E6

Major Metropolitan Centers Within Region:

\begin{tabular}{lcrl}
\multicolumn{1}{c}{ Center } & Population & \multicolumn{2}{c}{ Location } \\
Hartford SMSA & 750,000 & $67 \mathrm{~km}$ & NW \\
New Haven-West Haven SMSA & 430,000 & $64 \mathrm{~km}$ & W \\
New London-Norwich SMSA & 250,000 & $8 \mathrm{~km}$ & NNE \\
New Britain SMSA & 140,000 & $65 \mathrm{~km}$ & NW \\
Waterbury SMSA & 230,000 & $78 \mathrm{~km}$ & WNW
\end{tabular}

SITE-SPECIFIC DATA - AIRBORNE PATIWAYS

Average Annual State Production

of Crops and Animal Products

In $80-\mathrm{km}$ Radius Circle

Veg: $3.2 E 7$ kilogram

Milk: $4.4 E 8$ liter

Meat: $2.0 \mathrm{E} 7 \mathrm{ki}$ logram

Regional Productivity Factor:

Animal Grazing Factor:

0.6

0.6

Meteorology Period of Record: 1 JAN 74 - 31 DEC 74 Recovery: 95\%

SITE-SPECIFIC DATA - WATERBORNE PATHWAYS via NIANTIC BAY

Average Dilution Flow

from all 3 Plants: $3,900 \mathrm{ft}^{3} / \mathrm{s}$

Fish:

Edible Harvest: $9.1 \mathrm{E4} \mathrm{kg} / \mathrm{yr}$

Dilution Factor: 0.001

Invertebrates:

Edible Harvest: $9.1 E 4 \mathrm{~kg} / \mathrm{yr}$

Dilution Factor: 0.002 
POPULATION DOSE-COMMITMENT ESTIMATES AND

AVERAGE INDIVIDUAL DOSE-COMMITMENT HISTOGRAM FOR

MILLSTONE 1,2 , AND 3

Dose Commitments (person-rem) from Waterborne Pathways

Total Body GI-LLI Thyroid Bone Liver

$\begin{array}{llllll}\text { Infant } & 0.0 \mathrm{E}+00 & 0.0 \mathrm{E}+00 & 0.0 \mathrm{E}+00 & 0.0 \mathrm{E}+00 & 0.0 \mathrm{E}+00 \\ \text { Child } & 4.3 \mathrm{E}-04 & 7.1 \mathrm{E}-04 & 2.4 \mathrm{E}-04 & 6.0 \mathrm{E}-04 & 5.5 \mathrm{E}-04 \\ \text { Teen } & 3.0 \mathrm{E}-04 & 1.5 \mathrm{E}-03 & 1.7 \mathrm{E}-04 & 3.4 \mathrm{E}-04 & 4.5 \mathrm{E}-04 \\ \text { Aduit } & 1.9 \mathrm{E}-03 & 1.3 \mathrm{E}-02 & 1.1 \mathrm{E}-03 & 2.0 \mathrm{E}-03 & 2.7 \mathrm{E}-03 \\ \text { TOTAL } & 2.7 \mathrm{E}-03 & 1.5 \mathrm{E}-02 & 1.5 \mathrm{E}-03 & 3.0 \mathrm{E}-03 & 3.7 \mathrm{E}-03\end{array}$

Dose Commitments (person-rem) from Airborne Pathways

Total Body GI-LLI Thyroid Bone Liver Lung

$\begin{array}{lllllll}\text { Infant } & 3.4 \mathrm{E}-03 & 3.4 \mathrm{E}-03 & 9.4 \mathrm{E}-02 & 1.9 \mathrm{E}-03 & 3.7 \mathrm{E}-03 & 3.4 \mathrm{E}-03 \\ \text { Child } & 3.9 \mathrm{E}-02 & 3.8 \mathrm{E}-02 & 5.0 \mathrm{E}-01 & 2.0 \mathrm{E}-02 & 4.0 \mathrm{E}-02 & 4.0 \mathrm{E}-02 \\ \text { Teen } & 2.7 \mathrm{E}-02 & 2.7 \mathrm{E}-02 & 2.1 \mathrm{E}-01 & 1.4 \mathrm{E}-02 & 2.7 \mathrm{E}-02 & 2.8 \mathrm{E}-02 \\ \text { Adult } & 1.5 \mathrm{E}-01 & 1.5 \mathrm{E}-01 & 7.1 \mathrm{E}-01 & 8.0 \mathrm{E}-02 & 1.5 \mathrm{E}-01 & 1.6 \mathrm{E}-01 \\ \text { TOTAL } & 2.2 \mathrm{E}-01 & 2.2 \mathrm{E}-01 & 1.5 \mathrm{E}+00 & 1.2 \mathrm{E}-01 & 2.2 \mathrm{E}-01 & 2.3 \mathrm{E}-01\end{array}$

Production/Consumption factors:

Produce: $0.038 \quad$ Milk: 0.77 Meat: 0.059

Fraction of Population Receiving an Average Individual

Total-Body Dose Commitment from Alrborne Pathwaya

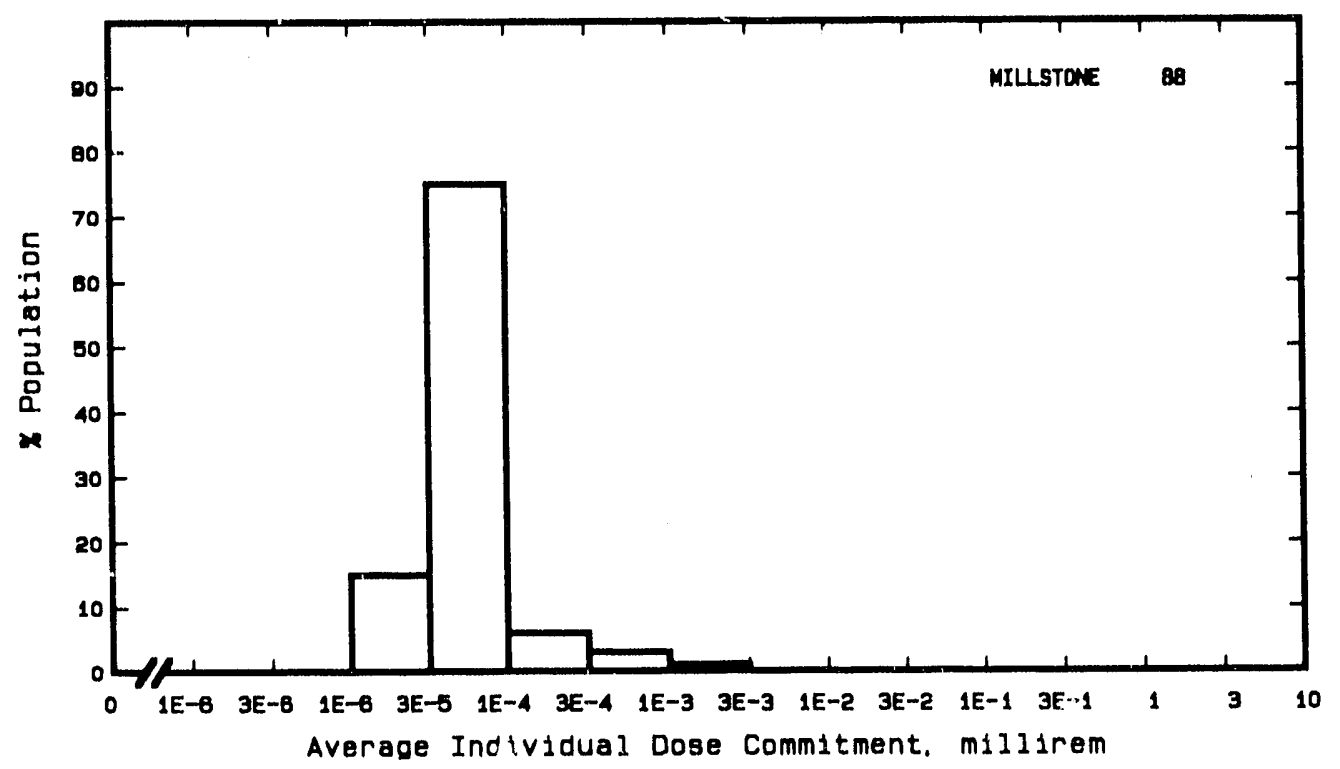


Site: MONTICELLO

MONTICELLO, MINNESO IA

Location: N $\quad$ N $45.3333^{\circ} \quad$ W $93.8483^{\circ}$

POPULATION DATA

Total Population Within 2-to-80-km Region: 2.3E6

Major Metropolitan Centers Within Region:

\section{Center}

Minneapolis-St. Paul SMSA

St. Cloud SMSA

Bloomington

Edina

Richfield
Population

$2,100,000$

160,000

85,000

48,000

40,000
Location

$60 \mathrm{~km} \quad \mathrm{SE}$

$36 \mathrm{~km} \quad \mathrm{NW}$

$72 \mathrm{~km} \quad \mathrm{SE}$

$63 \mathrm{~km} \quad \mathrm{SE}$

$67 \mathrm{~km} \quad \mathrm{SE}$

SITE-SPECIFIC DATA - AIRBORNE PATHWAYS

Average Annual State Production

of Crops and Animal Products

In $80-\mathrm{km}$ Radius Circle

Regional Productivity Factor:

Animal Grazing Factor:
Veg: $1.2 \mathrm{E} 8$ kilogram

Milk: 4.0E8 liter

Meat: 1.1E8 kilogram

1

0.5

Meteorology Period of Record: 1 JAN 74 - 31 DEC 74 Recovery: 92\%

SITE-SPECIFIC DATA - WATERBORNE PATHWAYS via MISSISSIPPI RIVER

Drinking Water:

Fish:
Average River Flow

at Site: $4,600 \mathrm{ft}^{3} / \mathrm{s}$

Exposed Population: None

Edible Harvest: None 


\section{POPULATION DOSE-COMMITMENT ESTIMATES AND \\ AVERAGE INDIVIDUAL DOSE-COMMITMENT HISTOGRAM FOR \\ MONTICELLO}

Dose Commitments (persori-rem) from Waterborne Pathways

Total Body GI-LLI Ihyroid Bone Liver

Infant.

Child

Teen

Adult

(No Waterborne Pathway Doses) (a)

TOTAL

Dose Commitments (person-rem) from Airborne Pathways

Total Body GI-LLI Thyroid Bone Liver Lung

$\begin{array}{lllllll}\text { Infant } & 2.7 \mathrm{E}-03 & 2.6 \mathrm{E}-03 & 6.7 \mathrm{E}-02 & 2.3 \mathrm{E}-03 & 2.9 \mathrm{E}-03 & 2.7 \mathrm{E}-03 \\ \text { Child } & 3.2 \mathrm{E}-02 & 3.1 \mathrm{E}-02 & 4.5 \mathrm{E}-01 & 2.7 \mathrm{E}-02 & 3.3 \mathrm{E}-02 & 3.2 \mathrm{E}-02 \\ \text { Teen } & 2.2 \mathrm{E}-02 & 2.2 \mathrm{E}-02 & 1.9 \mathrm{E}-01 & 1.8 \mathrm{E}-02 & 2.3 \mathrm{E}-02 & 2.3 \mathrm{E}-02 \\ \text { Adult } & 1.3 \mathrm{E}-01 & 1.3 \mathrm{E}-01 & 6.9 \mathrm{E}-01 & 1.0 \mathrm{E}-01 & 1.3 \mathrm{E}-01 & 1.3 \mathrm{E}-01 \\ \text { TOTAL } & 1.8 \mathrm{E}-01 & 1.8 \mathrm{E}-01 & 1.4 \mathrm{E}+00 & 1.5 \mathrm{E}-01 & 1.9 \mathrm{E}-01 & 1.9 \mathrm{E}-01\end{array}$

Production/Consumption factors:

Produce: $0.28 \quad$ Milk: $1.3 \quad$ Meat: 0.58

Fraction of Population Receiving an Average Indiyidual Total-Body Dose Commitment from Alrborne Pathways

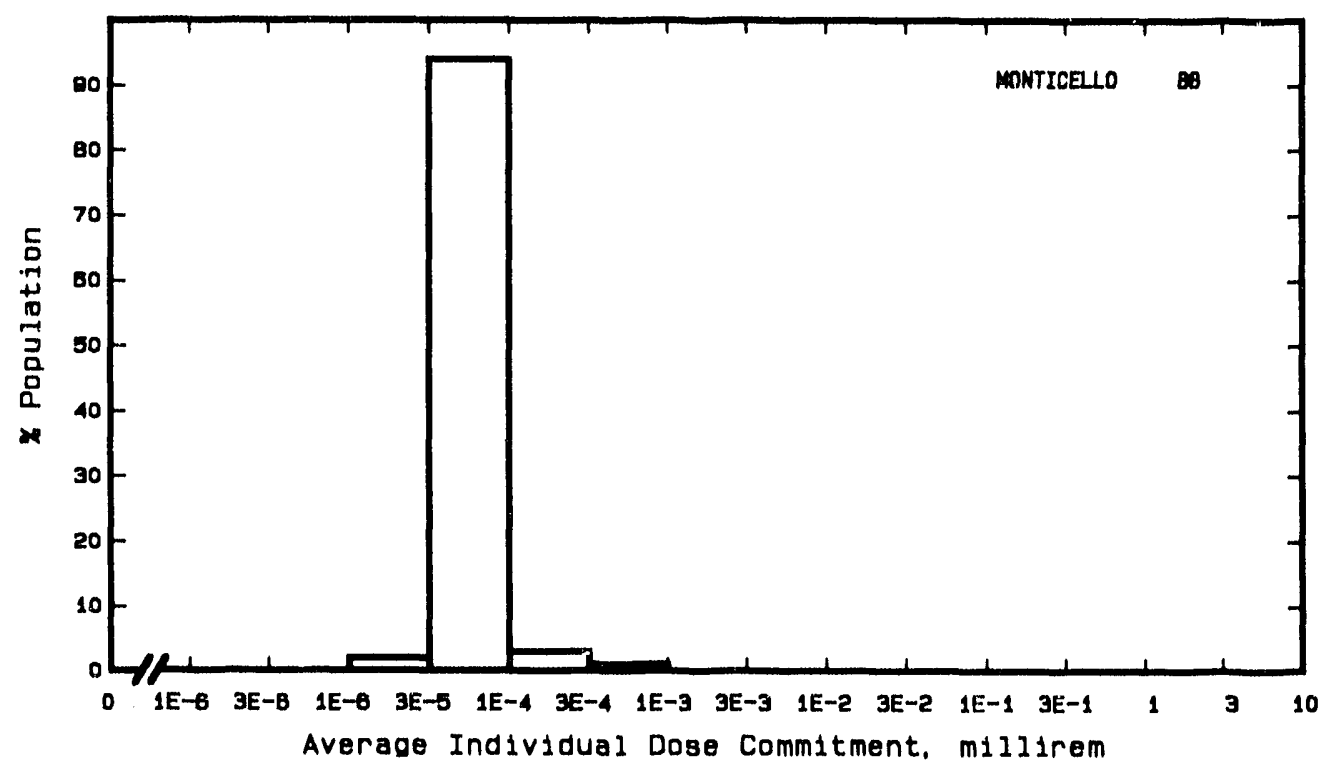

(a) No liquid releases reported (Tichler, et al. 1991). 
Site: NINE MILE POINT

OSWEGO, NEW YORK

Location: $\quad N 43.5222^{\circ} \quad$ W $76.4100^{\circ}$

POPULATION DATA

Total Population Within 2-to-80-km Region: 8.6 E5

Major Metropolitan Centers Within Region:

$\quad$ Center
Syracuse SMSA
Auburn
Watertown
Kingston
Oswego

Population

Location

680,000

33,000

28,000

24,000

20,000

$\begin{array}{lll}56 & \mathrm{~km} & \text { SSE } \\ 67 \mathrm{~km} & \text { SSW } \\ 64 \mathrm{~km} & \mathrm{NE} \\ 79 \mathrm{~km} & \mathrm{~N} \\ 11 \mathrm{~km} & \mathrm{SW}\end{array}$

SITE-SPECIFIC DATA - AIRBORNE PATHWAYS

Average Annual State Production

of Crops and Animal Products

In $80-\mathrm{km}$ Radius Circle

Veg: $7.6 \mathrm{E} 7$ kilogram

Milk: 7.0E8 liter

Regional Productivity Factor:

Animal Grazing Factor:

Meat: $3.3 \mathrm{E} 7$ kilogram

Meteorology Period of Record: 1 JAN 74 - 31 DEC 75 Recovery: 97\%

SITE-SPECIFIC DATA - WATERBORNE PATHWAYS via LAKE ONTARIO

$\begin{array}{ll} & \begin{array}{l}\text { Average Dilution } \mathrm{Fl} \text { low } \\ \text { from Plant: } 130 \mathrm{ft}^{3} / \mathrm{s}\end{array} \\ \text { Drinking Water: } & \text { Exposed Population: } 560,000 \\ & \text { Dilution Factor: } 0.01 \\ \text { Fish: } & \text { Edible Harvest: } 7.3 \mathrm{E} 5 \mathrm{~kg} / \mathrm{yr} \\ & \text { Dilution Factor: } 0.0033\end{array}$




\section{POPULATION DOSE-COMMITMENT ESTIMATES AND \\ AVERAGE INDIVIDUAL DOSE-COMMITMENT HISTOGRAM FOR \\ NINE MILE POINT 1 AND 2}

Dose Commitments (person-rem) from Waterborne Pathways

Total Body GI-LLI Thyroid Bone Liver

$\begin{array}{llllll}\text { Infant } & 3.6 \mathrm{E}-03 & 4.5 \mathrm{E}-03 & 3.1 \mathrm{E}-04 & 1.1 \mathrm{E}-03 & 4.5 \mathrm{E}-03 \\ \text { Child } & 5.2 \mathrm{E}-02 & 7.0 \mathrm{E}-02 & 3.4 \mathrm{E}-03 & 1.8 \mathrm{E}-02 & 6.1 \mathrm{E}-02 \\ \text { Teen } & 2.1 \mathrm{E}-02 & 7.1 \mathrm{E}-02 & 1.3 \mathrm{E}-03 & 7.9 \mathrm{E}-03 & 3.5 \mathrm{E}-02 \\ \text { Adult } & 1.3 \mathrm{E}-01 & 6.7 \mathrm{E}-01 & 1.1 \mathrm{E}-02 & 5.4 \mathrm{E}-02 & 2.2 \mathrm{E}-01 \\ \text { TOTAL } & 2.1 \mathrm{E}-01 & 8.2 \mathrm{E}-01 & 1.6 \mathrm{E}-02 & 8.0 \mathrm{E}-02 & 3.2 \mathrm{E}-01\end{array}$

Dose Commitments (person-rem) from Airborne Pathways

Iotal Body GI-LLI Thyroid Bone Liver Lung

$\begin{array}{lllllll}\text { Infant } & 6.3 \mathrm{E}-05 & 6.1 \mathrm{E}-05 & 7.5 \mathrm{E}-05 & 6.3 \mathrm{E}-05 & 9.4 \mathrm{E}-05 & 6.7 \mathrm{E}-05 \\ \text { Child } & 8.7 \mathrm{E}-04 & 8.1 \mathrm{E}-04 & 8.7 \mathrm{E}-04 & 9.3 \mathrm{E}-04 & 1.0 \mathrm{E}-03 & 8.7 \mathrm{E}-04 \\ \text { Teen } & 5.4 \mathrm{E}-04 & 5.3 \mathrm{E}-04 & 5.1 \mathrm{E}-04 & 4.3 \mathrm{E}-04 & 6.0 \mathrm{E}-04 & 5.7 \mathrm{E}-04 \\ \text { AduTl } & 2.9 \mathrm{E}-03 & 2.9 \mathrm{E}-03 & 2.7 \mathrm{E}-03 & 2.0 \mathrm{E}-03 & 2.9 \mathrm{E}-03 & 2.9 \mathrm{E}-03 \\ \text { TOTAL } & 4.4 \mathrm{E}-03 & 4.3 \mathrm{E}-03 & 4.2 \mathrm{E}-03 & 3.5 \mathrm{E}-03 & 4.7 \mathrm{E}-03 & 4.5 \mathrm{E}-03\end{array}$

Production/Consumption factors:

Produce: 0.32 Milk: $4.4 \quad$ Meat: 0.34

Fraction of Population Receiving an Average Individual Total-Body Dose Commitment from Alrborne Pathways

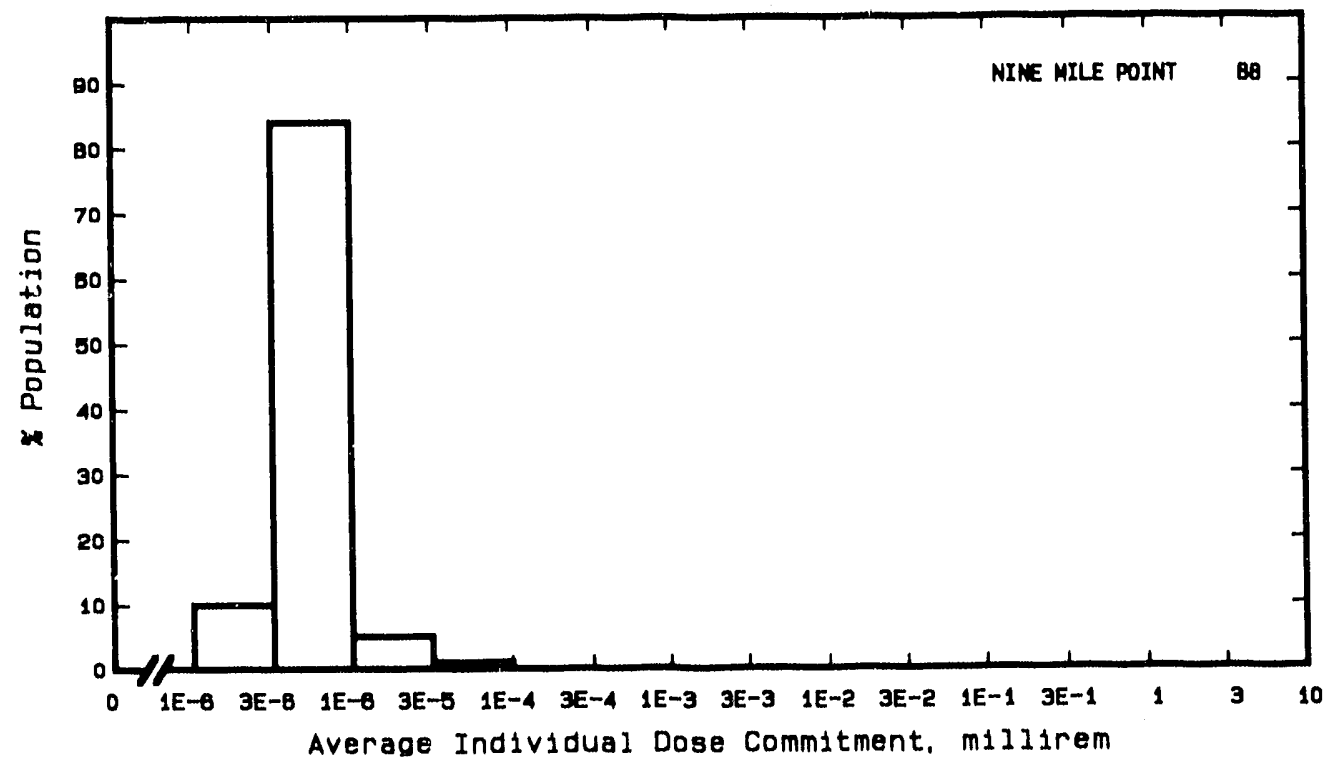


Site: NORTH ANNA

LOUISA COUNTY, VIRGINIA

Location: $\quad$ N $38.0608^{\circ} \quad$ W $77.7906^{\circ}$

POPULATION DATA

Total Population Within 2-to-80-km Region: 1.1E6

Major Metropolitan Centers Within Region:

Center

Richmond SMSA

Charlottesville

Fredricksburg

Culpeper

Ashl and
Population

680,000

44,000

16,000

7,000

5,000
Location

$66 \mathrm{~km} \quad$ SSE

$63 \mathrm{~km} \mathrm{~W}$

$40 \mathrm{~km} \quad \mathrm{NE}$

$54 \mathrm{~km} \quad \mathrm{NNW}$

$41 \mathrm{~km} \quad \mathrm{SE}$

SITE-SPECIFIC DATA - AIRBORNE PATHWAYS

Average Annual State Production

of Crops and Animal Products

In $80-\mathrm{km}$ Radius Circle

Regional Productivity Factor:

Animal Grazing Factor:
Veg: $3.5 \mathrm{E} 7$ kilogram

Milk: $1.5 \mathrm{E} 8$ liter

Meat: $7.4 \mathrm{E} 7$ kilogram

Meteorology Period of Record: 1 APR 74 - 31 APR 75 Recovery: 99\%

Average Dilution Flow from

Plants: $3,500 \mathrm{ft}^{3} / \mathrm{s}$

Drinking Water:

None

Fish:

Edible Harvest: $7.3^{(\mathrm{b})} \mathrm{kg} / \mathrm{yr}$

Dilution Factor: $0.001(\mathrm{~b})$

(a) Reconcentration of radionuclides in lake accounted for (FES 1973).

(b) Average individual consumption rates as given in FES (1973) were used in lieu of catch data. 
POPULATION DOSE-COMMITMENT ESTIMATES AND

AVERAGE INDIVIDUAL DOSE-COMMITMENT HISTOGRAM FOR

NORTH ANNA 1 AND 2

Dose Commitments (person-rem) from Waterborne Pathways

Total Body GI-LLI Thyroid Bone Liver

$\begin{array}{llllll}\text { Infant } & 0.0 \mathrm{E}+00 & 0.0 \mathrm{E}+00 & 0.0 \mathrm{E}+00 & 0.0 \mathrm{E}+00 & 0.0 \mathrm{E}+00 \\ \text { Child } & 4.6 \mathrm{E}-04 & 2.8 \mathrm{E}-04 & 1.2 \mathrm{E}-04 & 2.1 \mathrm{E}-03 & 2.4 \mathrm{E}-03 \\ \text { Teen } & 8.4 \mathrm{E}-04 & 5.3 \mathrm{E}-04 & 9.5 \mathrm{E}-05 & 1.3 \mathrm{E}-03 & 2.1 \mathrm{E}-03 \\ \text { Adult } & 8.9 \mathrm{E}-03 & 4.6 \mathrm{E}-03 & 6.8 \mathrm{E}-04 & 7.4 \mathrm{E}-03 & 1.2 \mathrm{E}-02 \\ \text { TOTAL } & 1.0 \mathrm{E}-02 & 5.4 \mathrm{E}-03 & 9.0 \mathrm{E}-04 & 1.1 \mathrm{E}-02 & 1.7 \mathrm{E}-02\end{array}$

Dose Commitments (person-rem) from Airborne Pathways

Total Body GI-LLI Thyroid Bone Liver Lung

$\begin{array}{lllllll}\text { Infant } & 3.8 \mathrm{E}-04 & 3.7 \mathrm{E}-04 & 1.9 \mathrm{E}-03 & 1.3 \mathrm{E}-04 & 4.5 \mathrm{E}-04 & 3.8 \mathrm{E}-04 \\ \text { Child } & 5.0 \mathrm{E}-03 & 4.9 \mathrm{E}-03 & 1.3 \mathrm{E}-02 & 1.3 \mathrm{E}-03 & 5.5 \mathrm{E}-03 & 5.1 \mathrm{E}-03 \\ \text { T?en } & 3.2 \mathrm{E}-03 & 3.1 \mathrm{E}-03 & 6.3 \mathrm{E}-03 & 7.4 \mathrm{E}-04 & 3.3 \mathrm{E}-03 & 3.2 \mathrm{E}-03 \\ \text { Adult } & 1.8 \mathrm{E}-02 & 1.7 \mathrm{E}-02 & 2.7 \mathrm{E}-02 & 3.9 \mathrm{E}-03 & 1.8 \mathrm{E}-02 & 1.8 \mathrm{E}-02 \\ \text { TOTAL } & 2.6 \mathrm{E}-02 & 2.5 \mathrm{E}-02 & 4.9 \mathrm{E}-02 & 6.1 \mathrm{E}-03 & 2.7 \mathrm{E}-02 & 2.6 \mathrm{E}-02\end{array}$

Production/Consumption factors:

Produce: 0.14

Milk: 0.90

Meat: 0.72

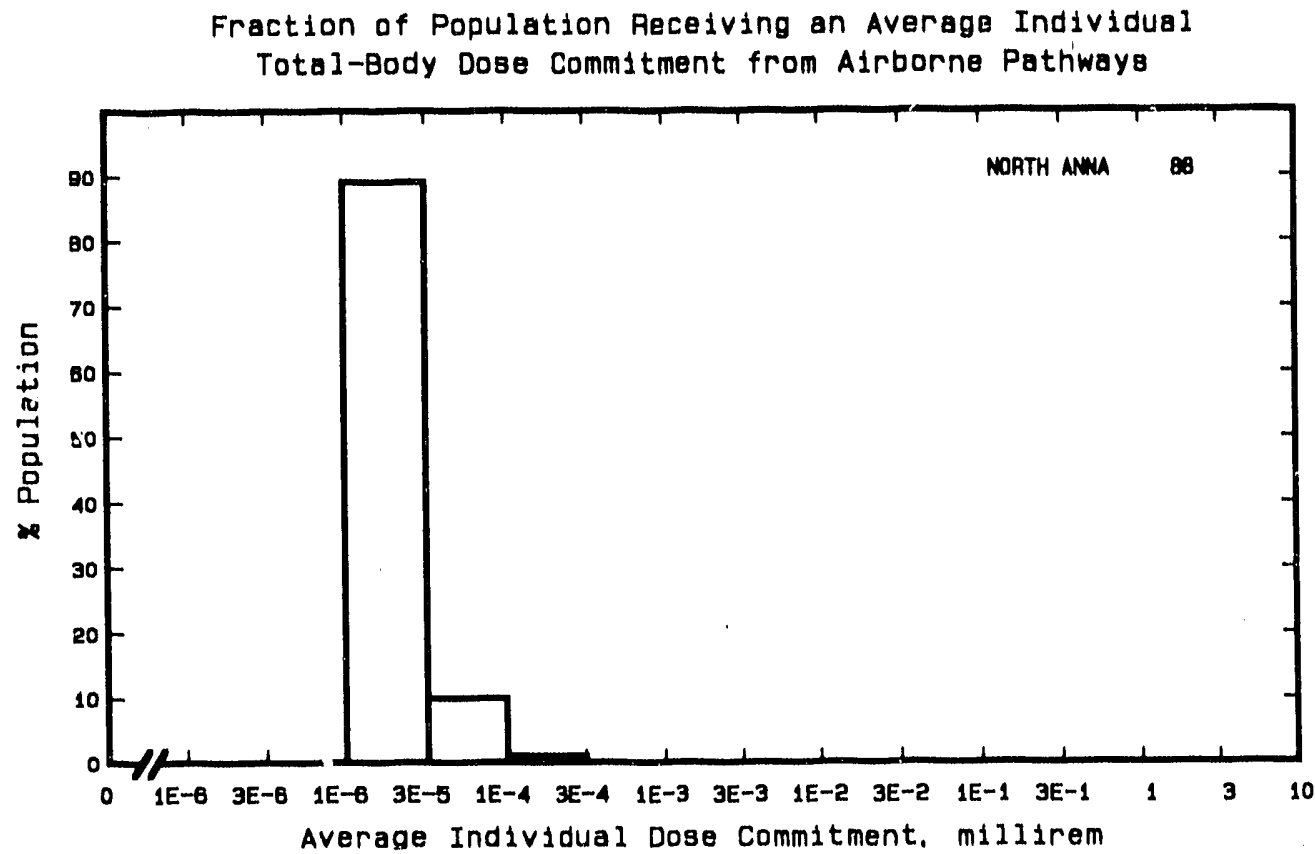


Site: OCONEE

OCONEE COUNTY, SOUTH CAROLINA

Location: $\quad$ N $34.7917^{\circ} \quad$ W $82.8986^{\circ}$

POPULATION DATA

Tota1 Population Within 2-to-80-km Region: 9.9E5

Major Metropolitan Centers Within Region:

Center

Greenville SMSA

Anderson

Easley

Greer
Population

630,000

29,000

15,000

12,000

\section{Location}

$46 \mathrm{~km} \quad \mathrm{E}$

$39 \mathrm{~km} \quad \mathrm{SI}$.

$27 \mathrm{~km} \quad \mathrm{~F}$.

$64 \mathrm{~km} \quad \mathrm{ENE}$

SITE-SPECIFIC DATA - AIRBORNE PATHWAYS

Average Annual State Production

of Crops and Animal Products

In $80-\mathrm{km}$ Radius Circle

Regional Productivity Factor:

Animal Grazing Factor:

Meteorology Period of Record: 1 JAN 75 - 31 DEC 75 Recovery: 86\%
Veg: 7.4E6 kilogram

Milk: 5.7E7 liter

Meat: $5.0 E 7$ kilogram

1

0.7

SITE-SPECIFIC DATA - WATERBORNE PATHWAYS via HARTWELL RES. On KEOWEE

RIVER

\section{Average River Flow}

at Site: $1,100 \mathrm{ft}^{3} / \mathrm{s}$

Drinking Water:

Exposed Population: 59,000

Dilution Factor: 1

Fish:

Edible Harvest: (a)

Dilution Factor: $0.01^{(b)}$

(a) No fish catch data given in FES, so generic consumption rates used (Table A-1).

(b) Ten percent of population obtain 10\% of their fish diet from Hartwell Reservoir according to FES (1972). 


\section{POPULATION DOSE-COMMITMENT ESTIMATES AND \\ AVERAGE INDIVIDUAL DOSE-COMMITMENT HISTOGRAM FOR}

OCONEE 1,2 , AND 3

Dose Commitments (person-rem) from Waterborne Pathways

Total Body GI-LII Ihyroid Bone Liver

$\begin{array}{llllll}\text { Infant } & 3.5 \mathrm{E}-02 & 4.0 \mathrm{E}-02 & 2.8 \mathrm{E}-01 & 1.3 \mathrm{E}-02 & 4.6 \mathrm{E}-02 \\ \text { Child } & 4.5 \mathrm{E}-01 & 5.8 \mathrm{E}-01 & 2.1 \mathrm{E}+00 & 4.1 \mathrm{E}-01 & 7.8 \mathrm{E}-01 \\ \text { Teen } & 2.6 \mathrm{E}-01 & 4.2 \mathrm{E}-01 & 6.7 \mathrm{E}-01 & 2.0 \mathrm{E}-01 & 4.4 \mathrm{E}-01 \\ \text { Adult } & 2.5 \mathrm{E}+00 & 3.7 \mathrm{E}+00 & 4.9 \mathrm{E}+00 & 1.2 \mathrm{E}+00 & 3.0 \mathrm{E}+00 \\ \text { TOTAL } & 3.3 \mathrm{E}+00 & 4.8 \mathrm{E}+00 & 7.9 \mathrm{E}+00 & 1.8 \mathrm{E}+00 & 4.3 \mathrm{E}+00\end{array}$

Dose Commitments (person-rem) from Airborne Pathways

Total Body GI-LII Thyroid Bone Liver Lung

$\begin{array}{llllllll}\text { Infant } & 7.7 \mathrm{E}-03 & 7.7 \mathrm{E}-03 & 3.4 \mathrm{E}-02 & 7.8 \mathrm{E}-03 & 7.9 \mathrm{E}-03 & 8.5 \mathrm{E}-03 \\ \text { Child } & 8.7 \mathrm{E}-02 & 8.6 \mathrm{E}-02 & 2.5 \mathrm{E}-01 & 8.7 \mathrm{E}-02 & 8.7 \mathrm{E}-02 & 9.8 \mathrm{E}-02 \\ \text { Teen } & 6.3 \mathrm{E}-02 & 6.3 \mathrm{E}-02 & 1.3 \mathrm{E}-01 & 6.2 \mathrm{E}-02 & 6.4 \mathrm{E}-02 & 7.8 \mathrm{E}-02 \\ \text { Adult } & 3.8 \mathrm{E}-01 & 3.8 \mathrm{E}-01 & 6.5 \mathrm{E}-01 & 3.7 \mathrm{E}-01 & 3.8 \mathrm{E}-01 & 4.3 \mathrm{E}-01 \\ \text { TOTAL } & 5.4 \mathrm{E}-01 & 5.4 \mathrm{E}-01 & 1.1 \mathrm{E}+00 & 5.3 \mathrm{E}-01 & 5.4 \mathrm{E}-01 & 6.1 \mathrm{E}-01\end{array}$

Production/Consumption factors:

Produce: 0.039 Milk: $0.44 \quad$ Meat: 0.63

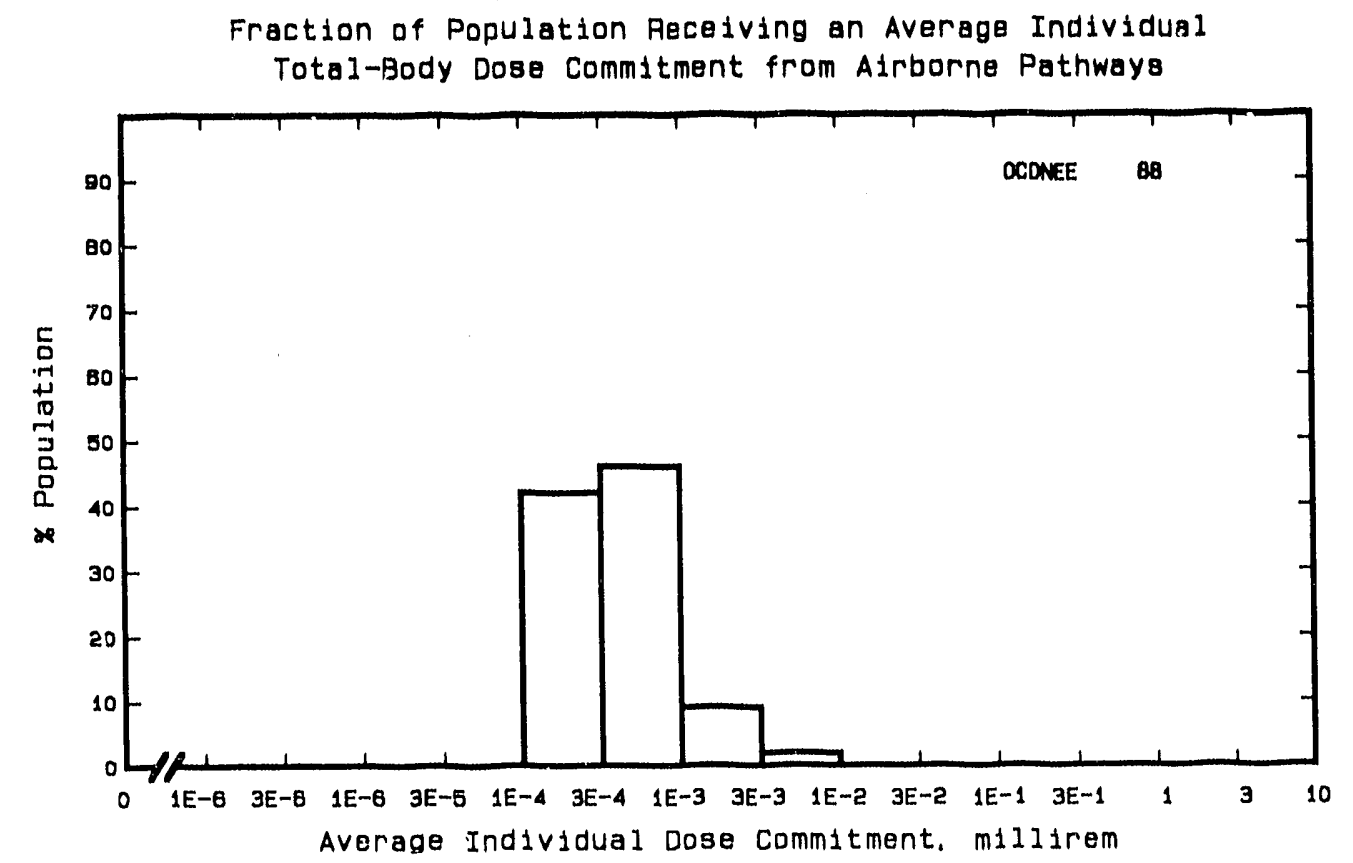


Site: OYSTER CREEK

OYSTER CREEK, NEW JERSEY

Location: $\quad$ N $39.8142^{\circ}$ W $74.2064^{\circ}$

POPULATION DATA

Total Population Within 2-to-80-km Region: 3.6E6

Major Metropolitan Centers Within Region:

Center Population Location

New Brunswick-Sayreville SMSA $640,000 \quad 77 \mathrm{~km} \quad \mathrm{~N}$

Long Branch-Asbury Park SMSA $520,000 \quad 57 \mathrm{~km}$ NNE

Trenton SMSA $\quad 320,000 \quad 66 \mathrm{~km} \quad \mathrm{NW}$

Atlantic City SMSA $\quad 190,000 \quad 55 \mathrm{~km} \quad$ SSW

Camden

88,000

$79 \mathrm{~km} \mathrm{~W}$

SITE-SPECIFIC DATA - AIRBORNE PATHWAYS

Average Annual State Production

of Crops and Animal Products

In $80-\mathrm{km}$ Radius Circle

Veg: $7.4 \mathrm{E} 7 \mathrm{kilogram}$

Milk: 2.7E8 Titer

Meat: $2.4 E 7$ kilogram

Regional Productivity Factor:

0.5

Animal Grazing Factor:

0.6

Meteorology Period of Record: 15 FEB 66 - 31 DEC 68 Recovery: $63 \%$

SITE-SPECIFIC. DATA - WATERBORNE PATHWAYS; via BARNEGAT BAY

Average Dilution Flow

from Plant: $26 \mathrm{ft}^{3} / \mathrm{s}$

Fish:

Edible Harvest: 2.1 (a) $\mathrm{kg} / \mathrm{yr}$

Dilution Factor: $0.01(\mathrm{~b})$

Invertebrates:

Edible Harvest: 0.96 (a) $\mathrm{kg} / \mathrm{yr}$

Dilution Factor: 0.01 (b)

(a) Average individual consumption rate as given in the FES (1974) used in lieu of catch data.

(b) $10 \%$ of seafood eaten assumed caught in bay waters diluted to $10 \%$ of that of discharge canal according to FES. 


\section{POPULATION DOSE-COMMITMENT ESTIMATES AND \\ AVERAGE INDIVIDUAL DOSE-COMMITMENT HISTOGRAM FOR \\ OYSTER CREEK}

Dose Commitments (person-rem) from Waterborne Pathways

Total Body GI-LII Ihyroid Bone Liver

$\begin{array}{llllll}\text { Infant } & 0.0 \mathrm{E}+00 & 0.0 \mathrm{E}+00 & 0.0 \mathrm{E}+00 & 0.0 \mathrm{E}+00 & 0.0 \mathrm{E}+00 \\ \text { Child } & 2.7 \mathrm{E}-02 & 4.6 \mathrm{E}-02 & 1.9 \mathrm{E}-03 & 1.7 \mathrm{E}-02 & 2.4 \mathrm{E}-02 \\ \text { Teen } & 2.1 \mathrm{E}-02 & 9.5 \mathrm{E}-02 & 1.5 \mathrm{E}-03 & 1.0 \mathrm{E}-02 & 2.0 \mathrm{E}-02 \\ \text { Adult } & 1.5 \mathrm{E}-01 & 8.4 \mathrm{E}-01 & 1.1 \mathrm{E}-02 & 5.9 \mathrm{E}-02 & 1.2 \mathrm{E}-01 \\ \text { TOTAL } & 2.0 \mathrm{E}-01 & 9.8 \mathrm{E}-01 & 1.4 \mathrm{E}-02 & 8.6 \mathrm{E}-02 & 1.7 \mathrm{E}-01\end{array}$

Dose Commitments (person-rem) from Airborne Pathways

Total Body GI-LII Thyroid Bone Liver Lung

$\begin{array}{lllllll}\text { Infant } & 2.9 \mathrm{E}-02 & 2.9 \mathrm{E}-02 & 6.1 \mathrm{E}-02 & 2.9 \mathrm{E}-02 & 2.9 \mathrm{E}-02 & 3.0 \mathrm{E}-02 \\ \text { Child } & 3.2 \mathrm{E}-01 & 3.2 \mathrm{E}-01 & 5.5 \mathrm{E}-01 & 3.2 \mathrm{E}-01 & 3.2 \mathrm{E}-01 & 3.3 \mathrm{E}-01 \\ \text { Teen } & 2.4 \mathrm{E}-01 & 2.4 \mathrm{E}-01 & 3.5 \mathrm{E}-01 & 2.3 \mathrm{E}-01 & 2.4 \mathrm{E}-01 & 2.5 \mathrm{E}-01 \\ \text { Adult } & 1.4 \mathrm{E}+00 & 1.4 \mathrm{E}+00 & 1.9 \mathrm{E}+00 & 1.4 \mathrm{E}+00 & 1.4 \mathrm{E}+00 & 1.5 \mathrm{E}+00 \\ \text { TOTAL } & 2.0 \mathrm{E}+00 & 2.0 \mathrm{E}+00 & 2.8 \mathrm{E}+00 & 2.0 \mathrm{E}+00 & 2.0 \mathrm{E}+00 & 2.1 \mathrm{E}+00\end{array}$

Production/Consumption factors:
Produce: 0.052
Milk: 0.28
Meat: 0.041

Fraction of Population Receiving an Average Individual

Total-Body Dose Commitment from Alrborne Pathways

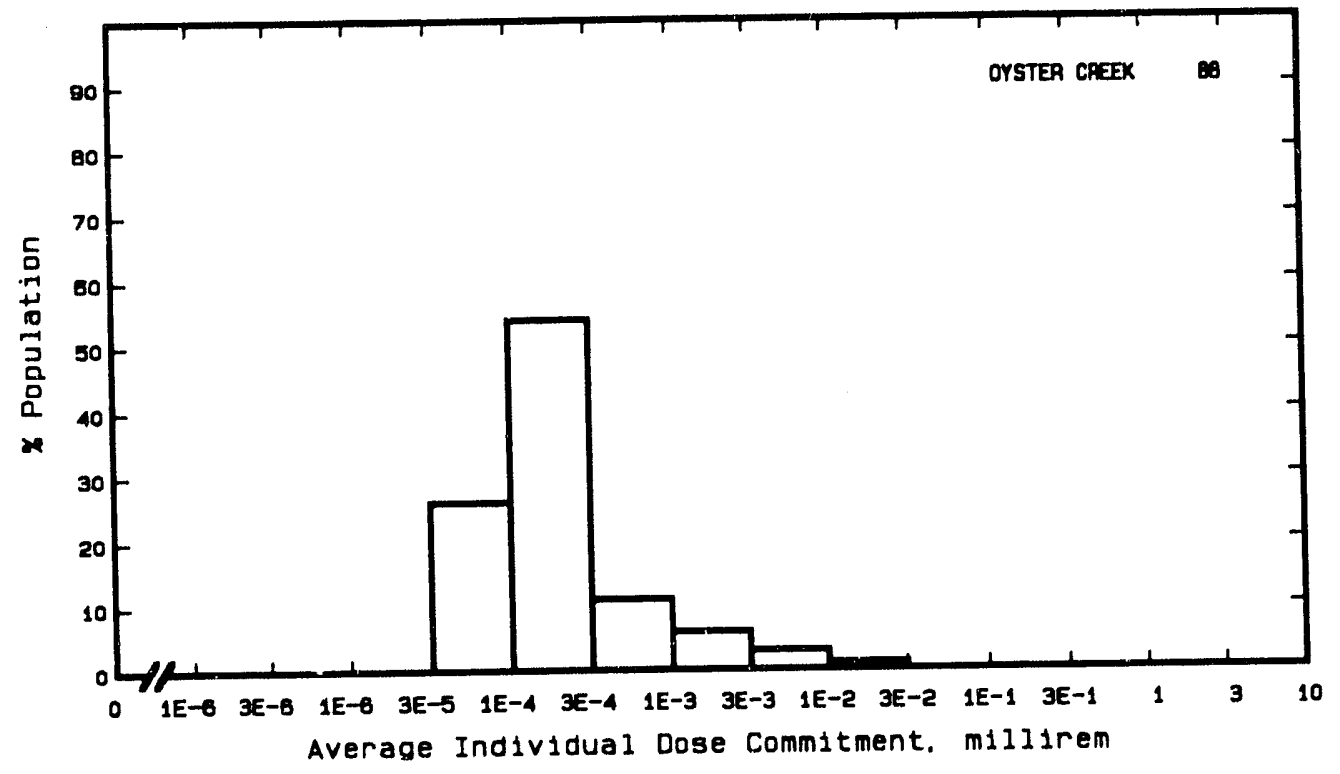


Site: PALISADES

Location: $\quad$ N $42.3222^{\circ}$
COVERT TOWNSHIP, MICHIGAN

W $86.3153^{\circ}$

POPULATION DATA

Total Population Within 2-to-80-km Region: 1.1E6

Major Metropolitan Centers Within Region:

Center

Kalamazoo-Portage SMSA

South Bend SMSA

Elkhart SMSA

Holl and

Benton Harbor
Population

280,000

280,000

140,000

26,000

15,000

\section{Location}

$61 \mathrm{~km} \quad \mathrm{E}$

$72 \mathrm{~km} \quad \mathrm{~S}$

$76 \mathrm{~km} \quad$ SSE

$53 \mathrm{~km}$ NNE

$25 \mathrm{~km} \quad \mathrm{SSW}$

\section{SITE-SPECIFIC DATA - AIRBORNE PATHWAYS}

Average Annual State Production

of Crops and Animal Products

In $80-\mathrm{km}$ Ridius Circle

Regional Productivity Factor:

Animal Grazing Factor:
Veg: $6.8 \mathrm{E} 7 \mathrm{ki}$ logram

Milk: 2.9E8 liter

Meat: $4.5 E 7$ kilogram

0.6

0.5

Meteorology Period of Record: 1 SEP 73 - 31 AUG 74 Recovery: 67\%

Average Dilution Flow from

Plant: $130 \mathrm{ft}^{3} / \mathrm{s}$

Drinking Water:

Exposed Population: 51,000

Dilution Factor: $3.5 \mathrm{E}-3(\mathrm{a})$

Fish:
Edible Harvest: (b)

Dilution Factor: 0.001

(a) Drinking water dilution factor estimated by averaying dilution factor derived from FES (1972) suitably weighted for population.

(b) Average individual consumption rate of $20 \mathrm{~g} / \mathrm{d}(7.3 \mathrm{~kg} / \mathrm{yr})$ as given in FES used in lieu of catch data. 


\section{POPULATION DOSE-COMMITMENT ESTIMATES AND AVERAGE INDIVIDUAL DOSE-COMMITMENT HISTOGRAM FOR \\ PALISADES}

Dose Commitments (person-rem) from Waterborne Pathways

Total Body GI-LII Ihyroid Bone Liver

$\begin{array}{llllll}\text { Infant } & 3.6 \mathrm{E}-04 & 3.4 \mathrm{E}-04 & 3.6 \mathrm{E}-04 & 1.2 \mathrm{E}-04 & 3.9 \mathrm{E}-04 \\ \text { Child } & 1.1 \mathrm{E}-02 & 4.4 \mathrm{E}-03 & 4.2 \mathrm{E}-03 & 5.0 \mathrm{E}-02 & 5.0 \mathrm{E}-02 \\ \text { Teen } & 1.5 \mathrm{E}-02 & 2.5 \mathrm{E}-03 & 1.7 \mathrm{E}-03 & 2.9 \mathrm{E}-02 & 3.9 \mathrm{E}-02 \\ \text { Adult } & 1.6 \mathrm{E}-01 & 2.1 \mathrm{E}-02 & 1.4 \mathrm{E}-02 & 1.7 \mathrm{E}-01 & 2.3 \mathrm{E}-01 \\ \text { TOTAL } & 1.9 \mathrm{E}-01 & 2.8 \mathrm{E}-02 & 2.1 \mathrm{E}-02 & 2.5 \mathrm{E}-01 & 3.2 \mathrm{E}-01\end{array}$

Dose Commitments (person-rem) from Airborne Pathways

Total Body GI-LII Ihyroid Bone Liver Lung

$\begin{array}{lllllll}\text { Infant } & 3.8 \mathrm{E}-04 & 3.6 \mathrm{E}-04 & 9.3 \mathrm{E}-03 & 3.8 \mathrm{E}-04 & 3.9 \mathrm{E}-04 & 4.0 \mathrm{E}-04 \\ \text { Child } & 4.2 \mathrm{E}-03 & 4.1 \mathrm{E}-03 & 5.7 \mathrm{E}-02 & 4.1 \mathrm{E}-03 & 4.2 \mathrm{E}-03 & 4.5 \mathrm{E}-03 \\ \text { Teen } & 3.0 \mathrm{E}-03 & 3.0 \mathrm{E}-03 & 2.4 \mathrm{E}-02 & 2.9 \mathrm{E}-03 & 3.0 \mathrm{E}-03 & 3.6 \mathrm{E}-03 \\ \text { Adult } & 1.8 \mathrm{E}-02 & 1.8 \mathrm{E}-02 & 8.5 \mathrm{E}-02 & 1.7 \mathrm{E}-02 & 1.8 \mathrm{E}-02 & 2.0 \mathrm{E}-02 \\ \text { TOTAL } & 2.5 \mathrm{E}-02 & 2.5 \mathrm{E}-02 & 1.8 \mathrm{E}-01 & 2.5 \mathrm{E}-02 & 2.6 \mathrm{E}-02 & 2.8 \mathrm{E}-02\end{array}$

Production/Consumption factors:

Produce: 0.20 Milk: 1.2 Meat: 0.32

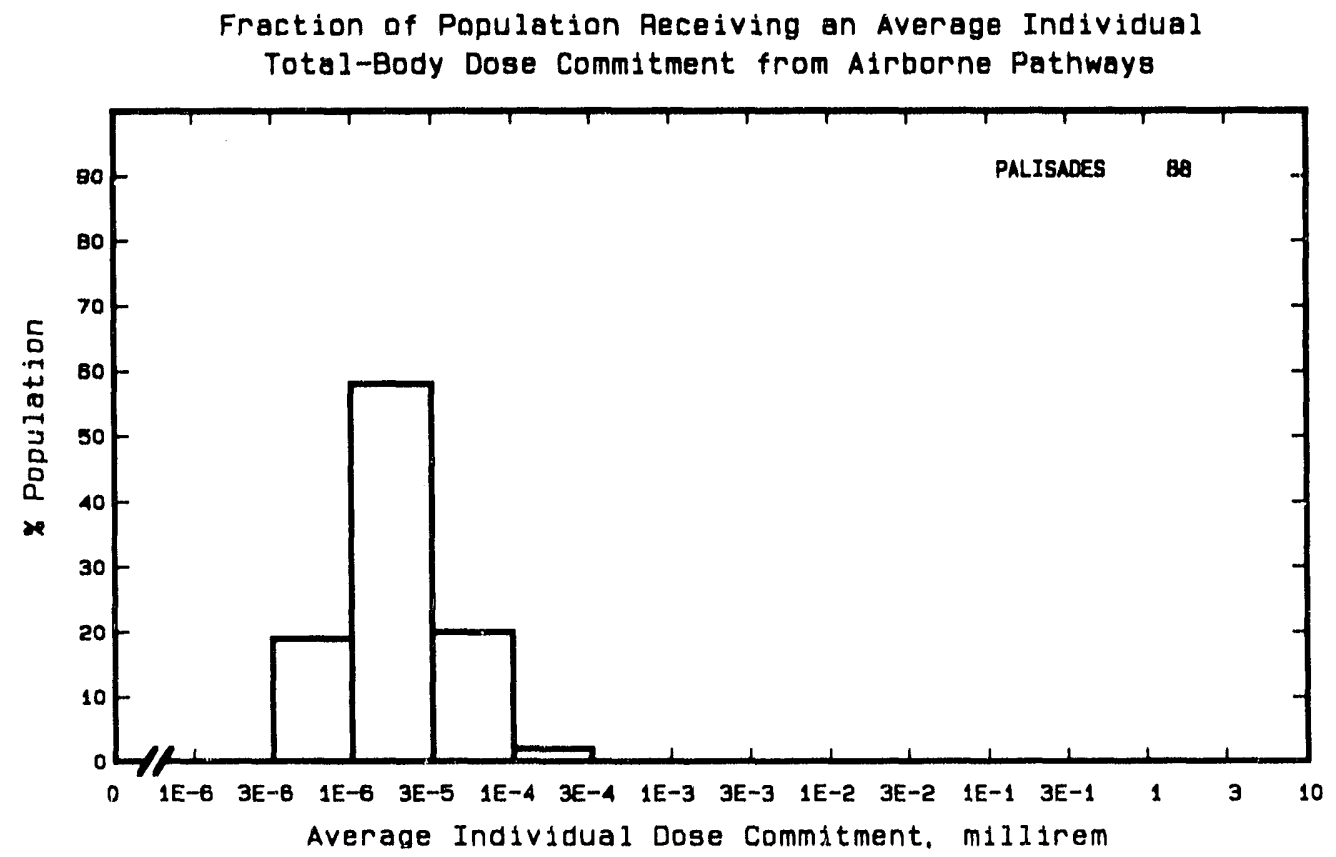


Site: PALO VERDE

Location: $\quad$ N $33.4200^{\circ}$
WINTERSBURG, ARIZONA

W $112.8683^{\circ}$

POPULATION DATA

Total Population Within 2-to-80-km Region: 1.2E6

Major Metropolitan Centers Within Region:

\begin{tabular}{ccccc} 
Center & Population & \multicolumn{2}{c}{ Location } \\
Phoenix MSA & $1,900,000$ & $64 \mathrm{~km}$ & $\mathrm{E}$ \\
Avondale & 8,200 & $49 \mathrm{~km}$ & $\mathrm{E}$
\end{tabular}

SITE-SPECIFIC DATA - AIRBORNE PATHWAYS

Average Annual State Production

of Crops and Animal Products

In $80-\mathrm{km}$ Radius Circle

Regional Productivity Factor:

Animal Grazing Factor:
Veg: $1.2 \mathrm{E} 7$ kilogram

Milk: 2.3E7 liter

Meat: $2.1 \mathrm{E} 7$ kilogram

0.9

$0.1 \operatorname{Mi} 1 k(a)$

0.9 Beef

Meteorology Period of Record: 13 AUG 73 - 8 AUG 78 Recovery: 93\%

SITE-SPECIFIC DATA - WATERBORNE PATHWAYS None

Average River Flow

at Site: (b)

Drinking Water:

None (b)

Fish:

None (b)

(a) Milk cows fed from dry lot $90 \%$ of time (FES 1975).

(b) No waterborne pathways 
POPULATION DOSE-COMMITMENT ESTIMATES AND

AVERAGE INDIVIDUAL DOSE-COMMITMENT HISTOGRAM FOR

PALO VERDE 1,2 , AND 3

Dose Commitments (person-rem) from Waterborne Pathways

Total Body GI-LLI Thyroid Bone Liver

Infant

Child

Teen

Adult

(No Waterborne Pathway Doses) (a)

TOTAL

Dose Commitments (person-rem) from Airborne Pathways

Total Body GI-LLI Thyroid Bone Liver Lung

Infant

2. $0 \mathrm{E}-03$

2. $0 \mathrm{E}-03$

3. $5 \mathrm{E}-03$

7. $3 \mathrm{E}-04$

2. $0 \mathrm{E}-03$

2. 1E-03

Child

3. $2 \mathrm{E}-02$

3.2E-02

$5.3 \mathrm{E}-02$

8.1E-03

$3.2 E-02$

3. 3E -02

Teen

2. 3E-02

2.3E-02

$3.4 \mathrm{E}-02$

$5.9 \mathrm{E}-03$

2. $3 \mathrm{E}-02$

2. 5E-02

Adult

$1.4 \mathrm{E}-01$

1. $4 \mathrm{E}-01$

1.9E-01

3. $6 \mathrm{E}-02$

1. $4 \mathrm{E}-01$

1. $4 \mathrm{E}-01$

TOTAL

2. $0 E-01$

2. $0 \mathrm{E}-01$

2.8E-01

5. 1E-02

2. $0 E-01$

2. $0 E-01$

Production/Consumption factors:

Produce: 0.045 Milk: 0.13 Meat: 0.20

Fraction of Population Peceiving an Average Individual

Tota1-Body Dose Comm1tment from Alrborne Pathways

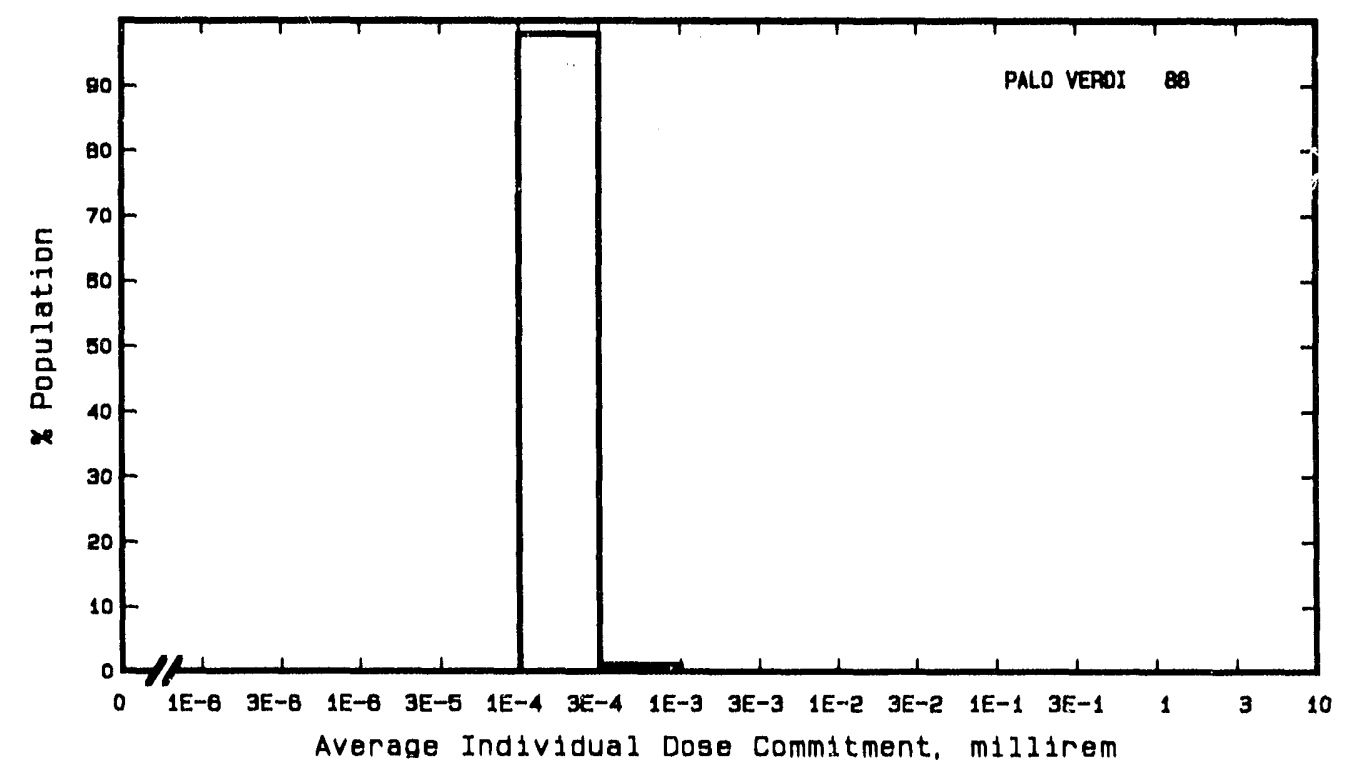

(a) No liquid releases reported (Tichler, et al. 1991) 
Site: PEACH BOTTOM

YORK COUNTY, PENNSYLVANIA

Location: N $39.7589^{\circ}$ W $76.2692^{\circ}$

POPULATION DATA

Total Population Within 2-to-80-km Region: 4.3E6

Major Metropolitan Centers Within Region:

Center

Baltimore SMSA

Harrisburg SMSA

Wilmington SMSA

York SMSA

Lancaster SMSA

\section{Population}

$2,200,000$

450,000

400,000

380,000

360,000
Location

$60 \mathrm{~km} \quad \mathrm{SSW}$

$77 \mathrm{~km} \quad \mathrm{NNW}$

$62 \mathrm{~km} \quad \mathrm{E}$

$45 \mathrm{~km} \quad \mathrm{NW}$

$31 \mathrm{~km} \mathrm{~N}$

SITE-SPECIFIC DATA - AIRBORNE PATHWAYS

Average Annual State Production

of Crops and Animal Products

In $80-\mathrm{km}$ Radius Circle

Regional Productivity Factor:

Animal Grazing Factor:
Veg: $5.3 \mathrm{E} 7 \mathrm{kilogram}$

Milk: 5.3E8 liter

Meat: 5.4E7 kilogram

0.95

0.6

Meteorology Period of Record: 1 AUG $67-31$ JUL 71 Recovery: $72 \%$

SITE-SPECIFIC DATA - WATERBORNE PATHWAYS via SUSQUEHANNA RIVER

Average River Flow

at Site: $36,000 \mathrm{ft}^{3} / \mathrm{s}$

Drinking Water:

Exposed Population: 2.2E6

Dilution Factor: 1

Fish:

Edible Harvest: (a)

Dilution Factor: $0.001^{(b)}$

(a) No fish catch data given in FES (1974), thus generic consumption rates used (Table A-1).

(b) One percent of people obtain $10 \%$ of their fish diet from river down-stream from plant according to FES. 


\section{POPULATION DOSE-COMMITMENT ESTIMATES AND \\ AVERAGE INDIVIDUAL DOSE-COMMITMENT HISTOGRAM FOR \\ PEACH BOTTOM 2 AND 3}

Dose Commitments (person-rem) from Waterborne Pathways

Total Body GI-LII Thyroid Bone Liver

$\begin{array}{llllll}\text { Infant } & 2.3 \mathrm{E}-03 & 1.0 \mathrm{E}-03 & 5.1 \mathrm{E}-04 & 1.2 \mathrm{E}-02 & 1.4 \mathrm{E}-02 \\ \text { Child } & 3.7 \mathrm{E}-02 & 1.0 \mathrm{E}-02 & 5.7 \mathrm{E}-03 & 1.4 \mathrm{E}-01 & 1.3 \mathrm{E}-01 \\ \text { Teen } & 2.4 \mathrm{E}-02 & 6.0 \mathrm{E}-03 & 2.2 \mathrm{E}-03 & 3.8 \mathrm{E}-02 & 4.9 \mathrm{E}-02 \\ \text { Adult } & 2.5 \mathrm{E}-01 & 5.4 \mathrm{E}-02 & 1.8 \mathrm{E}-02 & 2.6 \mathrm{E}-01 & 3.2 \mathrm{E}-01 \\ \text { TOTAL } & 3.2 \mathrm{E}-01 & 7.1 \mathrm{E}-02 & 2.7 \mathrm{E}-02 & 4.4 \mathrm{E}-01 & 5.1 \mathrm{E}-01\end{array}$

Dose Commitments (person-rem) from Airborne Pathways

Total Body GI-LII Thyroid Bone Liver Lung

$\begin{array}{lllllll}\text { Infant } & 2.5 \mathrm{E}-04 & 2.5 \mathrm{E}-04 & 2.2 \mathrm{E}-04 & 1.1 \mathrm{E}-04 & 3.0 \mathrm{E}-04 & 2.6 \mathrm{E}-04 \\ \text { Child } & 3.0 \mathrm{E}-03 & 2.7 \mathrm{E}-03 & 2.6 \mathrm{E}-03 & 1.7 \mathrm{E}-03 & 3.1 \mathrm{E}-03 & 3.3 \mathrm{E}-03 \\ \text { Teen } & 1.8 \mathrm{E}-03 & 1.7 \mathrm{E}-03 & 1.6 \mathrm{E}-03 & 9.4 \mathrm{E}-04 & 1.9 \mathrm{E}-03 & 2.2 \mathrm{E}-03 \\ \text { Adult } & 9.3 \mathrm{E}-03 & 9.0 \mathrm{E}-03 & 8.5 \mathrm{E}-03 & 4.6 \mathrm{E}-03 & 9.2 \mathrm{E}-03 & 1.1 \mathrm{E}-02 \\ \text { TOTAL } & 1.4 \mathrm{E}-02 & 1.4 \mathrm{E}-02 & 1.3 \mathrm{E}-02 & 7.3 \mathrm{E}-03 & 1.4 \mathrm{E}-02 & 1.7 \mathrm{E}-02\end{array}$

Production/Consumption factors:

Produce: $0.060 \quad$ Milk: 0.88 Meat: 0.15

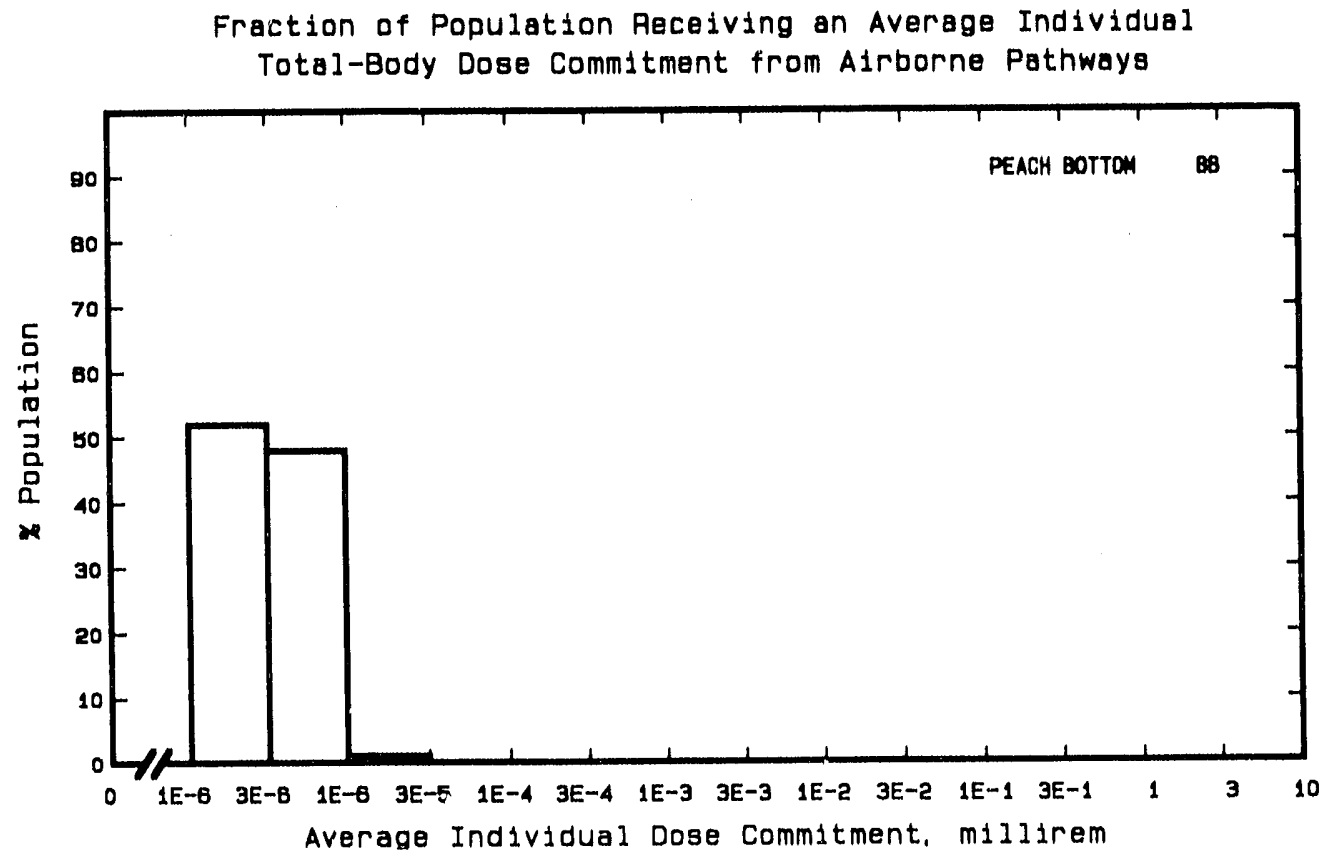


Site: PERRY

NORTH PERRY, OHIO

Location: $\quad$ N $41.8008^{\circ} \quad$ W $81.1433^{\circ}$

POPULATION DATA

Total Population Within 2-to-80-km Region: $2.4 \mathrm{E} 6$

Major Metropolitan Centers Within Region:

\begin{tabular}{lrrrr}
\multicolumn{1}{c}{ Center } & Population & \multicolumn{2}{c}{ Location } \\
\cline { 2 - 4 } & & $53 \mathrm{~km}$ & SW \\
Cleveland SMS & 580,000 & $80 \mathrm{~km}$ & SSW \\
Akron & 250,000 & $70 \mathrm{~km}$ & SE \\
Warren & 54,000 & $35 \mathrm{~km}$ & NE \\
Ashtabula & 24,000 & $11 \mathrm{~km}$ & SW
\end{tabular}

SITE-SPECIFIC DATA - AIRBORNE PATHWAYS

Average Annual State Production

of Crops and Animal Products

In $80-\mathrm{km}$ Radius Circle

Regional Productivity Factor:

Animal Grazing Factor:
Veg: $6.9 \mathrm{E7}$ kilogram

Milk: 3.7E6 liter

Meat: $1.2 E 8$ kilogram

0.45

0.5

Meteorology Period of Record: 1 MAY 72 - 31 AUG 78 Recovery: 92\%

Drinking Water:

Fish:
Average Dilution Flow
from Plant: $160 \mathrm{ft}^{3} / \mathrm{s}$

Exposed Population: $1,700,000$

Dilution Factor:

Edible Harvest: $1.2 \mathrm{E} 6 \mathrm{~kg} / \mathrm{yr}$

Dilution Factor: 0.016

(a) Estimated. 
POPULATION DOSE-COMMITMENT ESTIMATES AND

AVERAGE INDIVIDUAL DOSE-COMMITMENT HISTOGRAM FOR

PERRY

Dose Commitments (person-rem) from Waterborne Pathways

Total Body GI-LII Thyroid Bone Liver

$\begin{array}{llllll}\text { Infant } & 2.3 \mathrm{E}-03 & 2.3 \mathrm{E}-03 & 3.5 \mathrm{E}-02 & 3.0 \mathrm{E}-03 & 2.3 \mathrm{E}-03 \\ \text { Child } & 2.6 \mathrm{E}-02 & 3.4 \mathrm{E}-02 & 2.5 \mathrm{E}-01 & 3.3 \mathrm{E}-02 & 2.5 \mathrm{E}-02 \\ \text { Teen } & 9.6 \mathrm{E}-03 & 2.5 \mathrm{E}-02 & 7.4 \mathrm{E}-02 & 9.8 \mathrm{E}-03 & 1.0 \mathrm{E}-02 \\ \text { Adult } & 7.6 \mathrm{E}-02 & 2.3 \mathrm{E}-01 & 5.2 \mathrm{E}-01 & 6.6 \mathrm{E}-02 & 7.8 \mathrm{E}-02 \\ \text { TOTAL } & 1.1 \mathrm{E}-01 & 2.9 \mathrm{E}-01 & 8.7 \mathrm{E}-01 & 1.1 \mathrm{E}-01 & 1.1 \mathrm{E}-01\end{array}$

Dose Commitments (person-rem) from Airborne Pathways

Total Body GI-LII Thyroid Bone Liver Lung

$\begin{array}{lllllll}\text { Infant } & 9.4 \mathrm{E}-04 & 9.4 \mathrm{E}-04 & 4.3 \mathrm{E}-03 & 9.6 \mathrm{E}-04 & 9.5 \mathrm{E}-04 & 1.0 \mathrm{E}-03 \\ \text { Child } & 1.1 \mathrm{E}-02 & 1.1 \mathrm{E}-02 & 7.2 \mathrm{E}-02 & 1.2 \mathrm{E}-02 & 1.1 \mathrm{E}-02 & 1.1 \mathrm{E}-02 \\ \text { Teen } & 7.9 \mathrm{E}-03 & 7.8 \mathrm{E}-03 & 4.1 \mathrm{E}-02 & 8.4 \mathrm{E}-03 & 7.9 \mathrm{E}-03 & 8.8 \mathrm{E}-03 \\ \text { Adult } & 4.8 \mathrm{E}-02 & 4.7 \mathrm{E}-02 & 2.0 \mathrm{E}-01 & 5.0 \mathrm{E}-02 & 4.7 \mathrm{E}-02 & 5.0 \mathrm{E}-02 \\ \text { TOTAL } & 6.8 \mathrm{E}-02 & 6.7 \mathrm{E}-02 & 3.2 \mathrm{E}-01 & 7.2 \mathrm{E}-02 & 6.7 \mathrm{E}-02 & 7.2 \mathrm{E}-02\end{array}$

Production/Consumption factors:

Produce: 0.067 Milk: 0.0054 Meat: 0.27

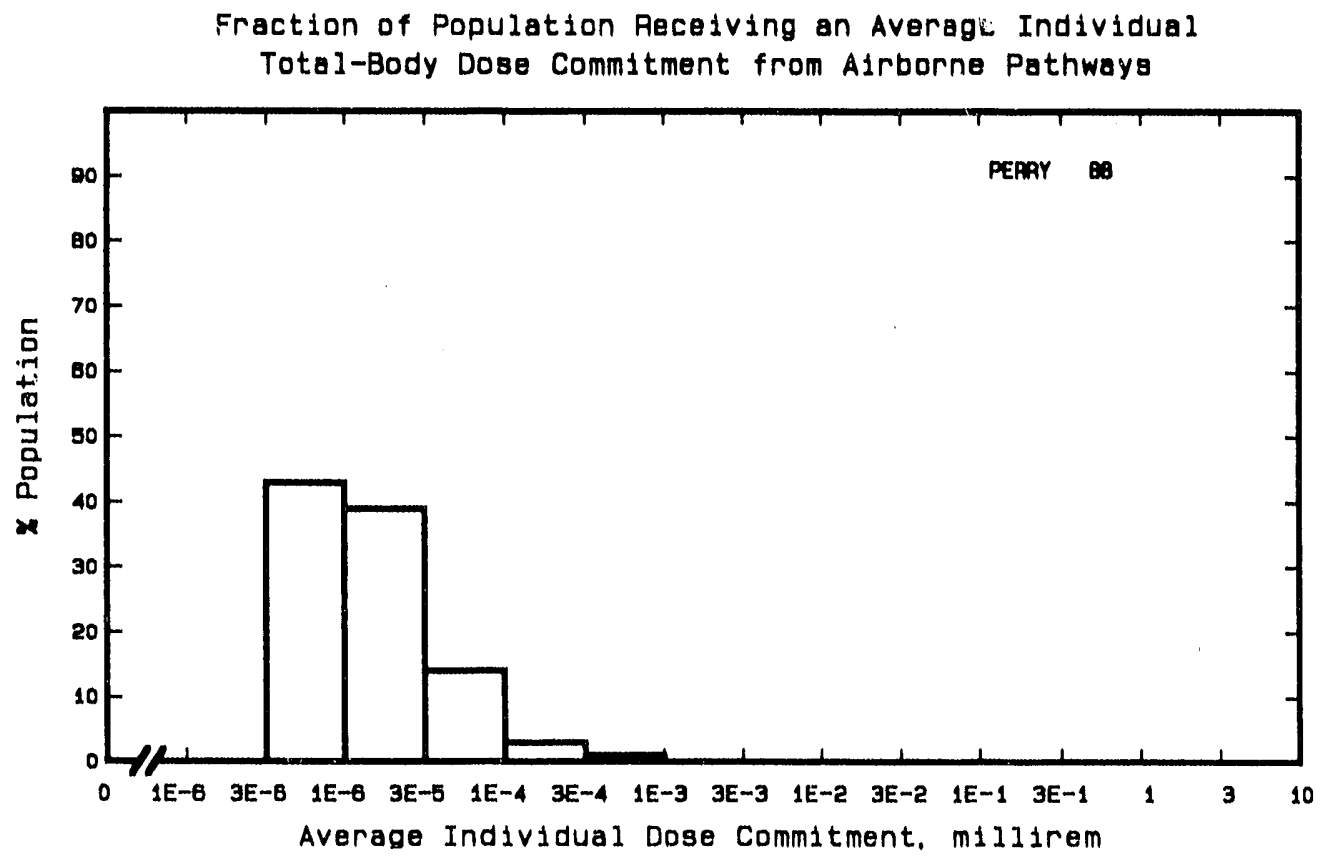


Site: PILGRIM

PLYMOUTH, MASSACHUSETTS

Location: $\quad$ N $41.9444^{\circ} \quad$ W $70.5794^{\circ}$

POPULATION DATA

Total Population Within 2-to-80-km Region: 4.4E6

Major Metropolitan Centers Within Region:

Center Population Location

$\begin{array}{lrrl}\text { Boston SMSA } & 2,800,000 & 61 \mathrm{~km} & \text { NW } \\ \text { Providence-Warwick-Pawtucket SMSA } & 840,000 & 70 \mathrm{~km} & \text { W } \\ \text { New Bedford SMSA } & 170,000 & 45 \mathrm{~km} & \text { SSW } \\ \text { Brockton SMSA } & 170,000 & 40 \mathrm{~km} & \text { WNW } \\ \text { Fall River SMSA } & 150,000 & 55 \mathrm{~km} & \text { SW }\end{array}$

SITE-SPECIFIC DATA - AIRBORNE PATHWAYS

Average Annual State Production

of Crops and Animal Products

In $80-\mathrm{km}$ Radius Circle

Veg: $2.0 \mathrm{E7}$ kilogram

Milk: 2.6E8 liter

Meat: $1.6 \mathrm{E} 7 \mathrm{kilogram}$

Regional Productivity Factor:

Animal Grazing Factor:

0.3

0.6

Meteorology Period of Record: 1 MAY 74 - 30 APR 75 Recovery: 93\%

SITE-SPECIFIC DATA - WATERBORNE PATHWAYS via CAPE COD BAY

Average Dilution Flow

from Plant: $6.0 \mathrm{ft}^{3} / \mathrm{s}$

Fish: $\quad$ Edible Harvest: $2.6 \mathrm{E} 4 \mathrm{~kg} / \mathrm{yr}$

Dilution Factor: 0.001

Invertebrates: $\quad$ Edible Harvest: $3.1 \mathrm{E4} \mathrm{kg} / \mathrm{yr}$

Dilution Factor: 0.002 
POPULATION DOSE-COMMITMENT ESTIMATES AND
AVERAGE INDIVIDUAL DOSE-COMMITMENT HISTOGRAM FOR

PILGRIM

Dose Commitments (person-rem) from Waterborne Pathways

Total Body GI-LII Thyroid Bone Liver

$\begin{array}{llllll}\text { Infant } & 0.0 \mathrm{E}+00 & 0.0 \mathrm{E}+00 & 0.0 \mathrm{E}+00 & 0.0 \mathrm{E}+00 & 0.0 \mathrm{E}+00 \\ \text { Child } & 1.9 \mathrm{E}-04 & 2.3 \mathrm{E}-04 & 1.1 \mathrm{E}-07 & 5.5 \mathrm{E}-04 & 4.0 \mathrm{E}-04 \\ \text { Teen } & 1.5 \mathrm{E}-04 & 4.7 \mathrm{E}-04 & 9.4 \mathrm{E}-08 & 3.1 \mathrm{E}-04 & 3.1 \mathrm{E}-04 \\ \text { Adult } & 1.1 \mathrm{E}-03 & 4.1 \mathrm{E}-03 & 7.5 \mathrm{E}-07 & 1.8 \mathrm{E}-03 & 1.8 \mathrm{E}-03 \\ \text { TOTAL } & 1.4 \mathrm{E}-03 & 4.8 \mathrm{E}-03 & 9.6 \mathrm{E}-07 & 2.7 \mathrm{E}-03 & 2.5 \mathrm{E}-03\end{array}$

Dose Commitments (person-rem) from Airborne Pathways

Iotal Body GI-LII Thyroid Bone Liver Lung

$\begin{array}{lllllll}\text { Infant } & 8.5 \mathrm{E}-06 & 8.3 \mathrm{E}-06 & 8.2 \mathrm{E}-06 & 9.8 \mathrm{E}-06 & 1.1 \mathrm{E}-05 & 1.4 \mathrm{E}-05 \\ \text { Child } & 9.8 \mathrm{E}-05 & 9.8 \mathrm{E}-05 & 9.5 \mathrm{E}-05 & 1.0 \mathrm{E}-04 & 1.1 \mathrm{E}-04 & 2.0 \mathrm{E}-04 \\ \text { Teen } & 7.3 \mathrm{E}-05 & 7.4 \mathrm{E}-05 & 7.0 \mathrm{E}-05 & 6.7 \mathrm{E}-05 & 7.8 \mathrm{E}-05 & 1.6 \mathrm{E}-04 \\ \text { Adult } & 4.4 \mathrm{E}-04 & 4.5 \mathrm{E}-04 & 4.2 \mathrm{E}-04 & 3.9 \mathrm{E}-04 & 4.4 \mathrm{E}-04 & 8.0 \mathrm{E}-04 \\ \text { TOTAL } & 6.2 \mathrm{E}-04 & 6.3 \mathrm{E}-04 & 5.9 \mathrm{E}-04 & 5.7 \mathrm{E}-04 & 6.4 \mathrm{E}-04 & 1.2 \mathrm{E}-03\end{array}$

Production/Consumption factors:

Produce: 0.0070 Milk: 0.13 Meat: 0.014

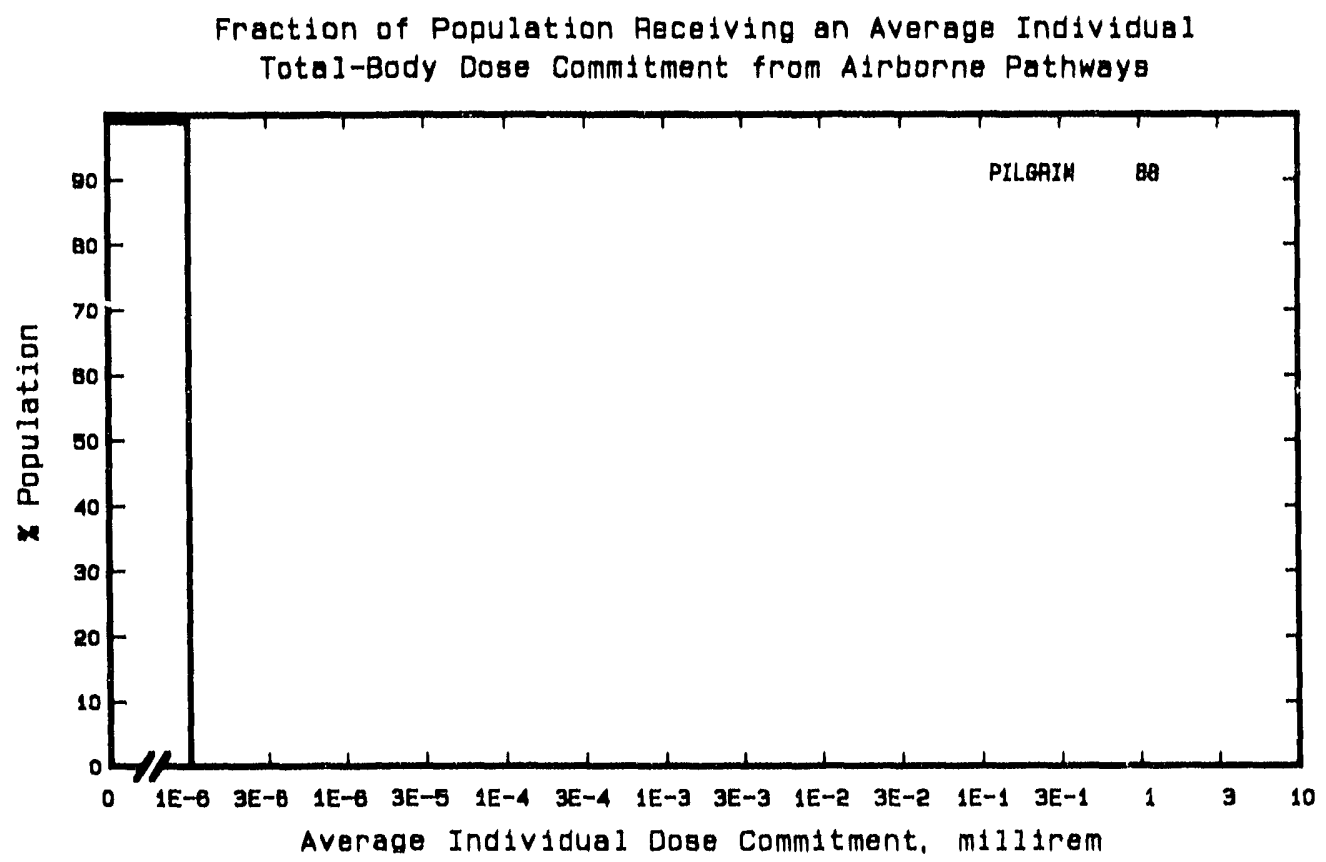


Site: POINT BEACH

Location: $\quad$ N $44.2808^{\circ} \quad$ W $87.5361^{\circ}$

POPULATION DATA

Total Population Within 2-to-80-km Region: 6.3E5

Major Metropolitan Centers Within Region:

Center

Greenbay SMSA

Appleton

Sheboygan

Manitowoc

Neenah
Population

180,000

61,000

49,000

34,000

22,000
Location

$47 \mathrm{~km} \quad \mathrm{NW}$

$72 \mathrm{~km} \mathrm{~W}$

$60 \mathrm{~km} \quad \mathrm{SSW}$

$24 \mathrm{~km} \quad \mathrm{SSW}$

$75 \mathrm{~km} \quad \mathrm{~W}$

\section{SITE-SPECIFIC DATA - AIRBORNE PATHWAYS}

Average Annual State Production

Of Crops and Animal Products

In $80-\mathrm{km}$ Radius Circle

Regional Productivity Factor:

Animal Grazing Factor:
Veg: 7.2E7 kilogram

Milk: 1.2Eg liter

Meat: 1.0E8 kilogram

Meteorology Period of Record: 19 APR 67 - 18 APR 69 Recovery: 83\%

Average Dilution Flow

from Plants: $770 \mathrm{ft}^{3} / \mathrm{s}$

Drinking Water:

Exposed Population: 270,000

Dilution Factor: $2.6 \mathrm{E}-3(\mathrm{a})$

Fish:

Edible Harvest: $6.7 \mathrm{E} 4$ (b) $\mathrm{kg} / \mathrm{yr}$

Dilution Factor: $0.013(\mathrm{c})$

(a) Drinking water dilution factor estimated by averaging dilution factor derived from FES (1972), suitably weighted for population.

(b) Includes both sport and commercial fish catch from FES.

(c) Dllution factor estimated by averaging sport and commerclal dilution factor derived from FES. 


\section{POPULATION DOSE-COMMITMENT ESTIMATES AND \\ AVERAGE INDIVIDUAL DOSE-COMMITMENT HISTOGRAM FOR \\ POINT BEACH 1 AND 2}

Dose Commitments (person-rem) from Waterborne Pathways

Total Body GI-LII Thyroid Bone Liver

$\begin{array}{llllll}\text { Infant } & 2.8 \mathrm{E}-04 & 2.7 \mathrm{E}-04 & 3.6 \mathrm{E}-04 & 4.5 \mathrm{E}-05 & 2.9 \mathrm{E}-04 \\ \text { Child } & 3.2 \mathrm{E}-03 & 3.1 \mathrm{E}-03 & 3.7 \mathrm{E}-03 & 9.9 \mathrm{E}-04 & 3.6 \mathrm{E}-03 \\ \text { Teen } & 1.3 \mathrm{E}-03 & 1.2 \mathrm{E}-03 & 1.3 \mathrm{E}-03 & 4.4 \mathrm{E}-04 & 1.6 \mathrm{E}-03 \\ \text { Adult } & 1.2 \mathrm{E}-02 & 1.1 \mathrm{E}-02 & 1.1 \mathrm{E}-02 & 2.7 \mathrm{E}-03 & 1.2 \mathrm{E}-02 \\ \text { TOTAL } & 1.7 \mathrm{E}-02 & 1.5 \mathrm{E}-02 & 1.7 \mathrm{E}-02 & 4.2 \mathrm{E}-03 & 1.8 \mathrm{E}-02\end{array}$

Dose Commitments (person-rem) from Airborne Pathways

Total Body GI-LII Thyroid Bone Liver Lung

$\begin{array}{lllllll}\text { Infant } & 3.8 \mathrm{E}-04 & 3.8 \mathrm{E}-04 & 5.5 \mathrm{E}-04 & 4.8 \mathrm{E}-05 & 4.1 \mathrm{E}-04 & 3.9 \mathrm{E}-04 \\ \text { Child } & 6.0 \mathrm{E}-03 & 5.9 \mathrm{E}-03 & 7.1 \mathrm{E}-03 & 5.3 \mathrm{E}-04 & 6.2 \mathrm{E}-03 & 6.0 \mathrm{E}-03 \\ \text { Teen } & 3.6 \mathrm{E}-03 & 3.6 \mathrm{E}-03 & 4.1 \mathrm{E}-03 & 2.6 \mathrm{E}-04 & 3.7 \mathrm{E}-03 & 3.7 \mathrm{E}-03 \\ \text { Adult } & 2.0 \mathrm{E}-02 & 2.0 \mathrm{E}-02 & 2.1 \mathrm{E}-02 & 1.3 \mathrm{E}-03 & 2.02 \mathrm{E}-02 & 2.0 \mathrm{E}-02 \\ \text { TOTAL } & 3.0 \mathrm{E}-02 & 3.0 \mathrm{E}-02 & 3.3 \mathrm{E}-02 & 2.1 \mathrm{E}-03 & 3.0 \mathrm{E}-02 & 3.0 \mathrm{E}-02\end{array}$

Production/Consumption factors:

Produce: 0.29 Milk: 7.0 Meat: 1.0

Fraction of Population Receiving an Average Individual

Total-Body Dose Commitment from Airborne Pathways

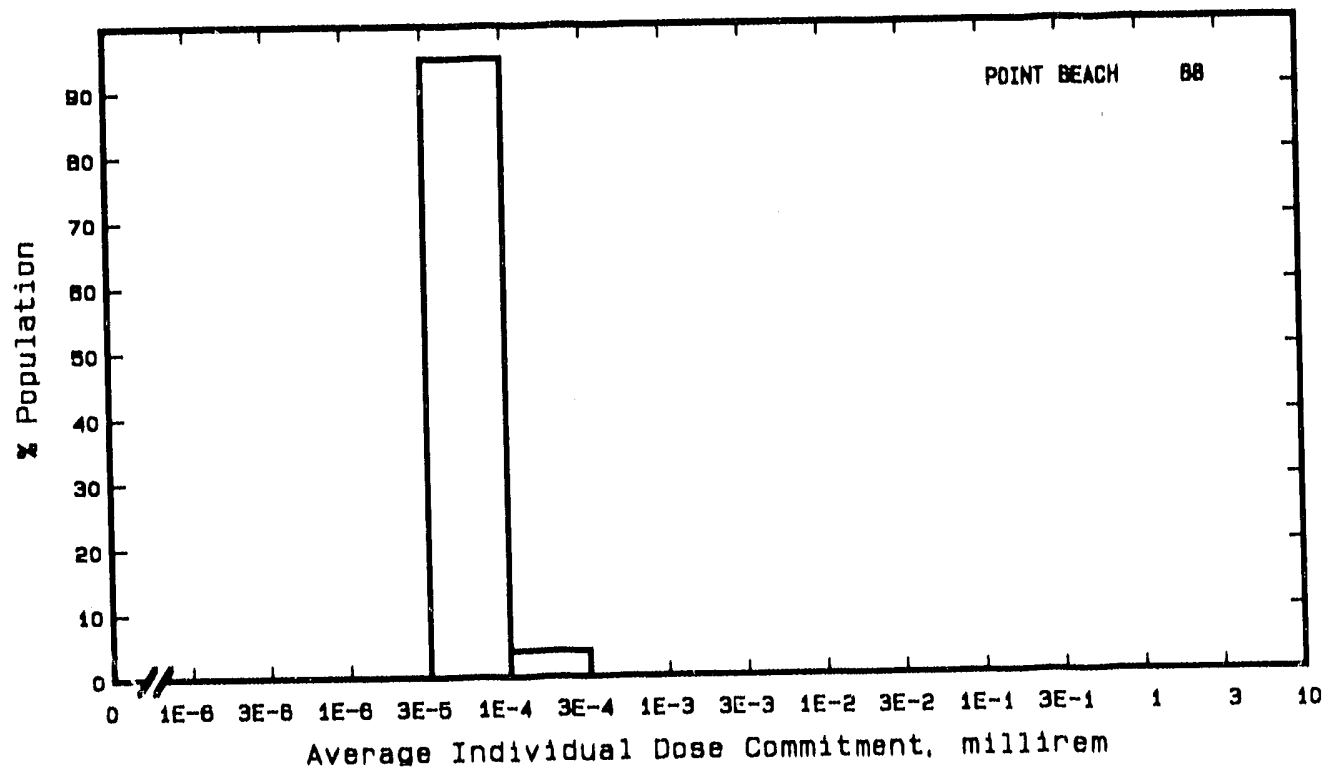


Site: PRAIRIE ISLAND

RED WING, MINNESOTA

Location: $\quad$ N $44.6219^{\circ} \quad$ W $92.6331^{\circ}$

POPULATION DATA

Total Population Within 2-to-80-km Region: 2.3E6

Major Metropolitan Centers Within Region:

\begin{tabular}{lrrrr}
\multicolumn{1}{c}{ Center } & Population & \multicolumn{2}{c}{ Location } \\
\cline { 2 - 5 } Minneapolis-St. Paul SMSA & $2,200,000$ & $63 \mathrm{~km}$ & NW \\
Rochester SMSA & 98,000 & $68 \mathrm{~km}$ & SSE \\
Owatonna & 19,000 & $77 \mathrm{~km}$ & SW \\
Faribault & 16,000 & $63 \mathrm{~km}$ & SW \\
Redwing & 14,000 & $10 \mathrm{~km}$ & SE
\end{tabular}

SITE-SPECIFIC DATA - AIRBORNE PATHWAYS

Average Annual State Production

Veg: $1.2 \mathrm{E} 8 \mathrm{ki}$ ogram

of Crops and Animal Products

In $80-\mathrm{km}$ Radius Circle

Milk: 4.0E8 liter

Meat: 1.0 E8 kilogram

Regional Productivity Factor:

Animal Grazing Factor:

1

0.5

Meteorology Period of Record: 22 MAR 74 - 21 MAR 75 Recovery: 65\%

SITE-SPECIFIC DATA - WATERBORNE PATHWAYS via MISSISSIPPI RIVER

Average River Flow

at Site: $15,000 \mathrm{ft}^{3} / \mathrm{s}$

Drinking Water: None

Fish:

Edible Harvest: $6.8 \mathrm{E} 5 \mathrm{~kg} / \mathrm{yr}$

Dilution Factor: 1 


\section{POPULATION DOSE-COMMITMENT ESTIMATES AND AVERAGE INDIVIDUAL DOSE-COMMITMENT HISTOGRAM FOR \\ PRAIRIE ISLAND 1 AND 2}

Dose Commitments (person-rem) from Waterborne Pathways

Total Body GI-LII Thyroid Bone Liver

$\begin{array}{llllll}\text { Infant } & 0.0 \mathrm{E}+00 & 0.0 \mathrm{E}+00 & 0.0 \mathrm{E}+00 & 0.0 \mathrm{E}+00 & 0.0 \mathrm{E}+00 \\ \text { Child } & 4.2 \mathrm{E}-04 & 7.6 \mathrm{E}-04 & 2.3 \mathrm{E}-04 & 1.2 \mathrm{E}-03 & 6.9 \mathrm{E}-04 \\ \text { Teen } & 3.3 \mathrm{E}-04 & 1.3 \mathrm{E}-03 & 2.0 \mathrm{E}-04 & 6.9 \mathrm{E}-04 & 5.6 \mathrm{E}-04 \\ \text { Adult } & 2.4 \mathrm{E}-03 & 1.1 \mathrm{E}-02 & 1.6 \mathrm{E}-03 & 4.1 \mathrm{E}-03 & 3.7 \mathrm{E}-03 \\ \text { TOTAL } & 3.1 \mathrm{E}-03 & 1.3 \mathrm{E}-02 & 2.1 \mathrm{E}-03 & 6.0 \mathrm{E}-03 & 4.9 \mathrm{E}-03\end{array}$

Dose Commitments (person-rem) from Airborne Pathways

Total Body GI-LII Thyroid Bone Liver Lung

$\begin{array}{lllllll}\text { Infant } & 9.6 \mathrm{E}-04 & 9.6 \mathrm{E}-04 & 9.6 \mathrm{E}-04 & 4.5 \mathrm{E}-06 & 9.6 \mathrm{E}-04 & 9.6 \mathrm{E}-04 \\ \text { Child } & 1.5 \mathrm{E}-02 & 1.5 \mathrm{E}-02 & 1.5 \mathrm{E}-02 & 1.8 \mathrm{E}-04 & 1.5 \mathrm{E}-02 & 1.5 \mathrm{E}-02 \\ \text { Teen } & 9.4 \mathrm{E}-03 & 9.4 \mathrm{E}-03 & 9.4 \mathrm{E}-03 & 6.8 \mathrm{E}-05 & 9.4 \mathrm{E}-03 & 9.4 \mathrm{E}-03 \\ \text { Adult } & 5.1 \mathrm{E}-02 & 5.1 \mathrm{E}-02 & 5.1 \mathrm{E}-02 & 2.7 \mathrm{E}-04 & 5.1 \mathrm{E}-02 & 5.1 \mathrm{E}-02 \\ \text { TOTAL } & 7.7 \mathrm{E}-02 & 7.6 \mathrm{E}-02 & 7.7 \mathrm{E}-02 & 5.2 \mathrm{E}-04 & 7.6 \mathrm{E}-02 & 7.6 \mathrm{E}-02\end{array}$

Production/Consumption factors:

Produce: $0.27 \quad$ Milk: $1.3 \quad$ Meat: 0.57

Fraction of Population Receiving an Average Individual

Total-Bouy Dose Commitment frofi Airborne Pathwoys

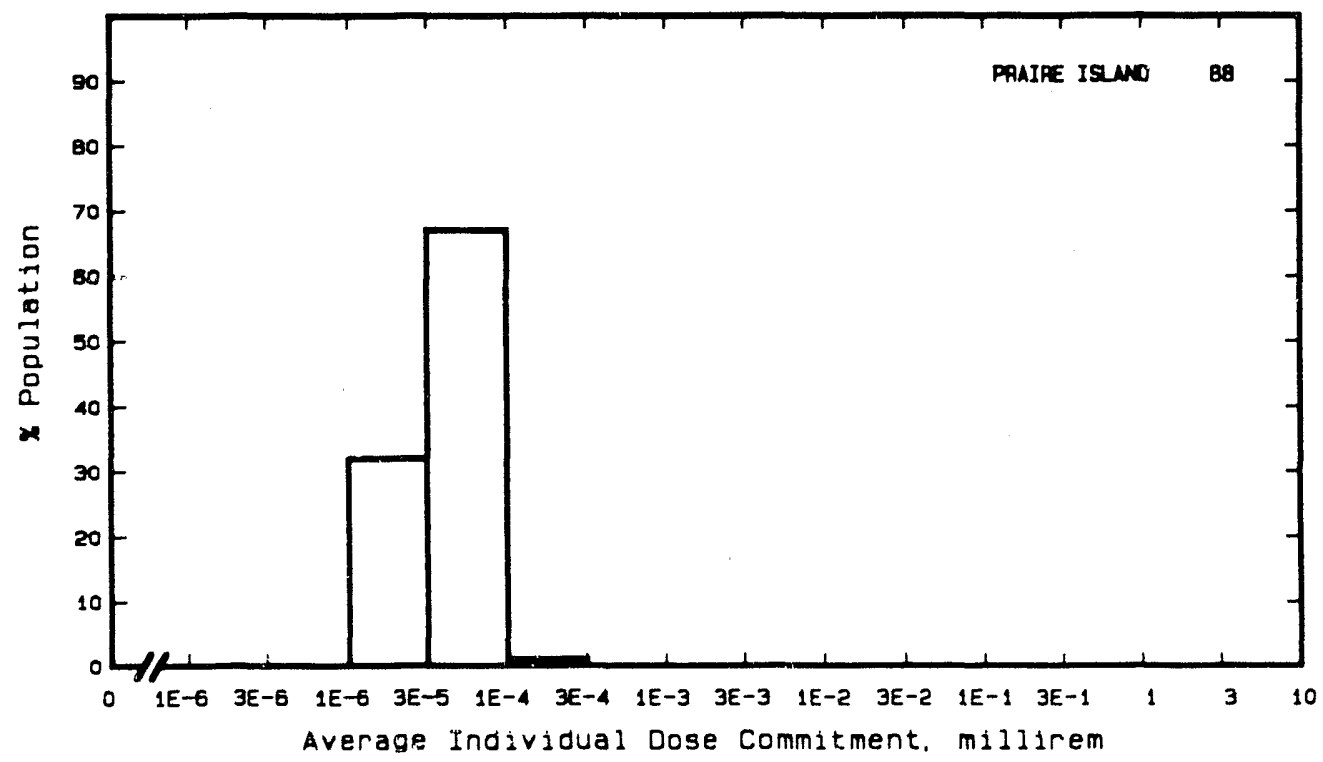


Site: QUAD CITIES

ROCK ISLAND, ILLINOIS

Location: N $41.7261^{\circ} \quad$ W $90.3100^{\circ}$

POPULATION DATA

Total Population Within 2-to-80-km Region: 7.3E's

Major Metropolitan Centers Within Region:

Center Population Location

$\begin{array}{lrrl}\text { Davenport-Rock Island-Mol ine SMSA } & 390,000 & 30 \mathrm{~km} & \text { SW } \\ \text { Muscatine } & 23,000 & 70 \mathrm{~km} & \text { WSW } \\ \text { Sterling } & 16,000 & 52 \mathrm{~km} & \text { E } \\ \text { Dixon } & 16,000 & 70 \mathrm{~km} & \text { E } \\ \text { Kewanee } & 15,000 & 62 \mathrm{~km} & \text { SSE }\end{array}$

SITE-SPECIr : DATA - AIRBORNE PATHWAYS

Average Annual State Production

of Crops and Animal Products

In $80-\mathrm{km}$ Radius Circle

Regional Productivity Factor:

Animal Grazing Factor:

Meteorology Period of Record: 1 JAN 74 - 31 DEC 75 Recovery: 88\%
Veg: $1.1 \mathrm{E} 8$ kilogram

Milk: 1.8E8 liter

Meat: $1.9 \mathrm{E} 8$ kilogram

1

0.5

SITE-SPECIFIC DATA - WATERBORNE PATHWAYS via MISSISSIPPI RIVER

Average River Flow

at Site: $47,000 \mathrm{ft}^{3} / \mathrm{s}$

Drinking Water:

Exposed Population: 390,000 (a)

Dilution Factor: 1

Fish:

Edible Harvest: $2.1 \mathrm{Eg}$ (b) $\mathrm{kg} / \mathrm{yr}$

Dilution Factor: 0.5 (b)

(a) All people in Davenport SMSA assumed to drink river water.

(b) Assumes $1 / 2$ fish harvest caught below plant. 
POPULATION DOSE-COMMITMENT ESTIMATES AND

AVERAGE INDIVIDUAL DOSE-COMMITMENT HISTOGRAM FOR

QUAD CITIES 1 AND 2

Dose Commitments (person-rem) from Waterborne Pathways

Total Body GI-LII Thyroid Bone Liver

$\begin{array}{llllll}\text { Infant } & 9.5 \mathrm{E}-05 & 6.6 \mathrm{E}-05 & 5.7 \mathrm{E}-05 & 2.4 \mathrm{E}-04 & 1.9 \mathrm{E}-04 \\ \text { Child } & 2.7 \mathrm{E}-03 & 9.7 \mathrm{E}-04 & 6.2 \mathrm{E}-04 & 1.3 \mathrm{E}-02 & 1.1 \mathrm{E}-02 \\ \text { Teen } & 3.2 \mathrm{E}-(13 & 7.1 \mathrm{E}-04 & 2.3 \mathrm{E}-04 & 6.6 \mathrm{E}-03 & 8.2 \mathrm{E}-03 \\ \text { Adult } & 3.4 \mathrm{E}-02 & 6.2 \mathrm{E}-03 & 2.0 \mathrm{E}-03 & 3.9 \mathrm{E}-02 & 4.9 \mathrm{E}-02 \\ \text { TOTAL } & 4.0 \mathrm{E}-02 & 8.0 \mathrm{E}-03 & 2.9 \mathrm{E}-03 & 5.9 \mathrm{E}-02 & 6.8 \mathrm{E}-02\end{array}$

Dose Commitments (person-rem) from Airborne Pathways

Total Body GI-LII Thyroid Bone Liver Lung

$\begin{array}{lllllll}\text { Infant } & 1.7 \mathrm{E}-04 & 1.7 \mathrm{E}-04 & 2.5 \mathrm{E}-03 & 1.0 \mathrm{E}-04 & 2.2 \mathrm{E}-04 & 2.1 \mathrm{E}-04 \\ \text { Child } & 3.2 \mathrm{E}-03 & 3.3 \mathrm{E}-03 & 2.5 \mathrm{E}-02 & 1.6 \mathrm{E}-03 & 3.6 \mathrm{E}-03 & 3.8 \mathrm{E}-03 \\ \text { Teen } & 2.0 \mathrm{E}-03 & 2.2 \mathrm{E}-03 & 1.1 \mathrm{E}-02 & 7.7 \mathrm{E}-04 & 2.1 \mathrm{E}-03 & 2.5 \mathrm{E}-03 \\ \text { Adult } & 1.1 \mathrm{E}-02 & 1.2 \mathrm{E}-02 & 4.3 \mathrm{E}-02 & 3.9 \mathrm{E}-03 & 1.1 \mathrm{E}-02 & 1.3 \mathrm{E}-02 \\ \text { TOTAL } & 1.6 \mathrm{E}-02 & 1.8 \mathrm{E}-02 & 8.1 \mathrm{E}-02 & 6.3 \mathrm{E}-03 & 1.7 \mathrm{E}-02 & 2.0 \mathrm{E}-02\end{array}$

Production/Consumption factors:

Produce: $0.80 \quad$ Milk: $1.8 \quad$ Meat: 3.2

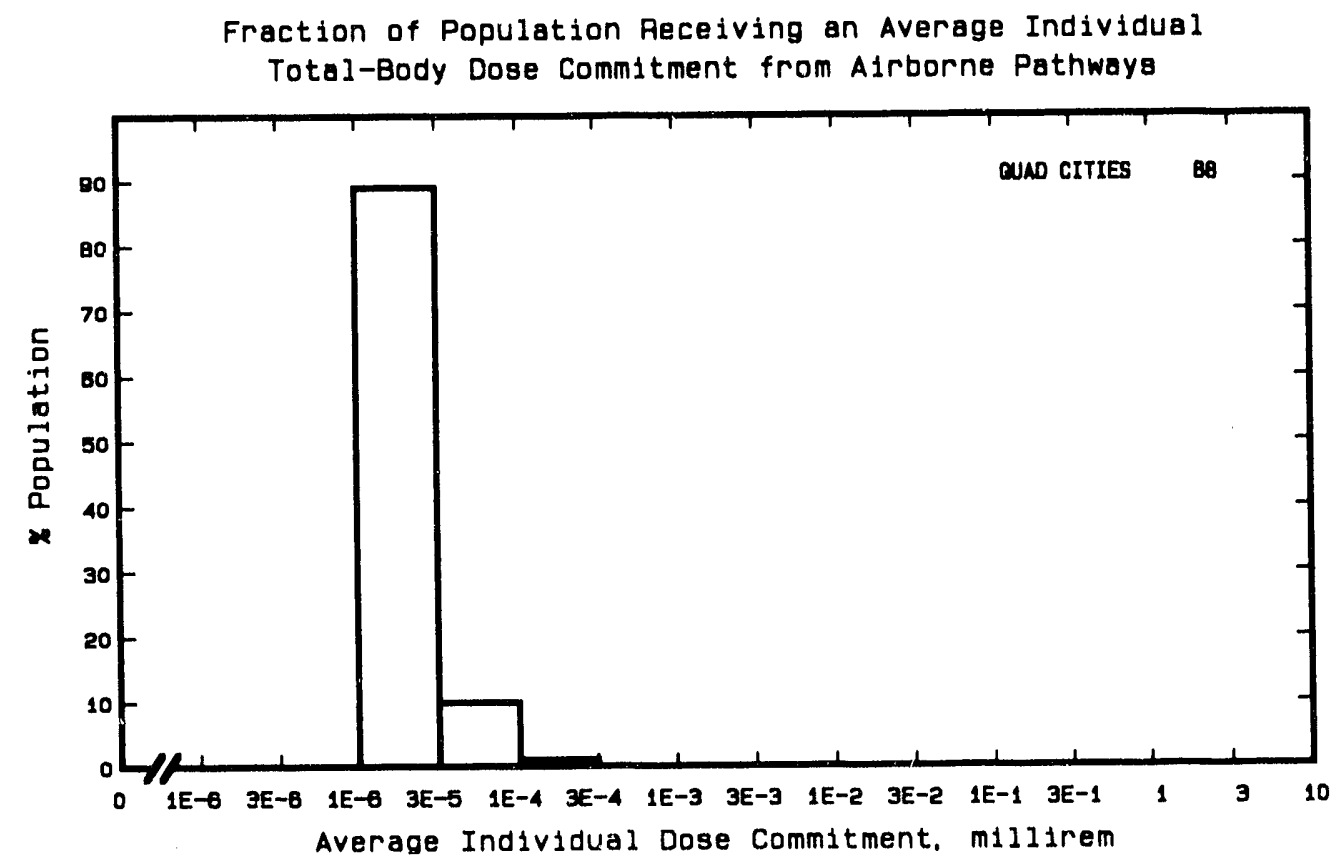


Site: RANCHO SECO

SACRAMENTO COUNTY, CALIFORNIA

Location: $\quad N \quad 38.3444^{\circ} \quad$ W $121.1200^{\circ}$

POPULATION DATA

Total Population Within 2-to-80-km Region: 2.1E6

Major Metropolitan Centers Within Region:

\begin{tabular}{lrrrr}
\multicolumn{1}{c}{ Center } & Population & \multicolumn{2}{c}{ Location } \\
Sacramento SMSA & $1,300,000$ & & \\
Stockton SMSA & 440,000 & $42 \mathrm{~km}$ & NW \\
Modesto SMSA & 330,000 & $45 \mathrm{~km}$ & SSW \\
Antioch & 50,000 & $79 \mathrm{~km}$ & S \\
Davis & 43,000 & $71 \mathrm{~km}$ & WSW \\
Davis & & $58 \mathrm{~km}$ & WNW
\end{tabular}

SITE-SPECIFIC DATA - AIRBORNE PATHWAYS

Average Annual State Production

of Crops and Animal Products

In $80-\mathrm{km}$ Radius Circle

Veg: $4.8 \mathrm{E} 7 \mathrm{kilogram}$

Milk: 2.3E8 liter

Meat: $5.0 \mathrm{E} 7 \mathrm{kilogram}$

Regional Productivity Factor:

Animal Grazing Factor:

1

0.9

Meteorology Period of Record: 1 FEB 75 - 31 JAN 76 Recovery: 98\%

SITE SPECIFIC DATA - WATERBORNE PATHWAYS via COSUMNES and MOKELUMNE

RIVERS

Average Dilution Flow

from Plant: $19 \mathrm{ft}^{3} / \mathrm{s}$

Drinking Water: None

Fish:

Edible Harvest: (a)

Dilution Factor: 0.005

(a) One percent of population is assumed to obtain fish from river. Average individual consumption rate of $7.3 \mathrm{~kg} / \mathrm{yr}$ given in FES (1973) used in lieu of catch data. 


\section{POPULATION DOSE-COMMITMENT ESTIMATES AND \\ AVERAGE INDIVIDUAL DOSE-COMMITMENT HISTOGRAM FOR \\ RANCHO SECO}

Dose Commitments (person-rem) from Waterborne Pathways

Total Body GI-LII Ihyroid Bone Liver

$\begin{array}{llllll}\text { Infant } & 0.0 \mathrm{E}+00 & 0.0 \mathrm{E}+00 & 0.0 \mathrm{E}+00 & 0.0 \mathrm{E}+00 & 0.0 \mathrm{E}+00 \\ \text { Child } & 9.0 \mathrm{E}-04 & 8.5 \mathrm{E}-05 & 1.6 \mathrm{E}-04 & 5.2 \mathrm{E}-03 & 5.4 \mathrm{E}-03 \\ \text { Teen } & 1.7 \mathrm{E}-03 & 1.2 \mathrm{E}-04 & 1.2 \mathrm{E}-04 & 3.1 \mathrm{E}-03 & 4.5 \mathrm{E}-03 \\ \text { Adult } & 1.8 \mathrm{E}-02 & 9.4 \mathrm{E}-04 & 8.6 \mathrm{E}-04 & 1.8 \mathrm{E}-02 & 2.7 \mathrm{E}-02 \\ \text { TOTAL } & 2.1 \mathrm{E}-02 & 1.1 \mathrm{E}-03 & 1.1 \mathrm{E}-03 & 2.6 \mathrm{E}-02 & 3.6 \mathrm{E}-02\end{array}$

Dose Commitments (person-rem) from Airborne Pathways

Total Body GI-LII Thyroid Bone Liver Lung

$\begin{array}{lllllll}\text { Infant } & 8.5 \mathrm{E}-04 & 8.5 \mathrm{E}-04 & 1.1 \mathrm{E}-03 & 7.2 \mathrm{E}-04 & 8.5 \mathrm{E}-04 & 9.1 \mathrm{E}-04 \\ \text { Child } & 9.7 \mathrm{E}-03 & 9.7 \mathrm{E}-03 & 1.1 \mathrm{E}-02 & 8.0 \mathrm{E}-03 & 9.7 \mathrm{E}-03 & 1.1 \mathrm{E}-02 \\ \text { Teen } & 7.0 \mathrm{E}-03 & 7.0 \mathrm{E}-03 & 7.5 \mathrm{E}-03 & 5.9 \mathrm{E}-03 & 7.0 \mathrm{E}-03 & 8.1 \mathrm{E}-03 \\ \text { Adult } & 4.1 \mathrm{E}-02 & 4.1 \mathrm{E}-02 & 4.3 \mathrm{E}-02 & 3.5 \mathrm{E}-02 & 4.1 \mathrm{E}-02 & 4.5 \mathrm{E}-02 \\ \text { TOTAL } & 5.9 \mathrm{E}-02 & 5.9 \mathrm{E}-02 & 6.3 \mathrm{E}-02 & 5.0 \mathrm{E}-02 & 5.9 \mathrm{E}-02 & 6.5 \mathrm{E}-02\end{array}$

Production/Consumption factors:

Produce: 0.12 Milk: 0.84 Meat: 0.30

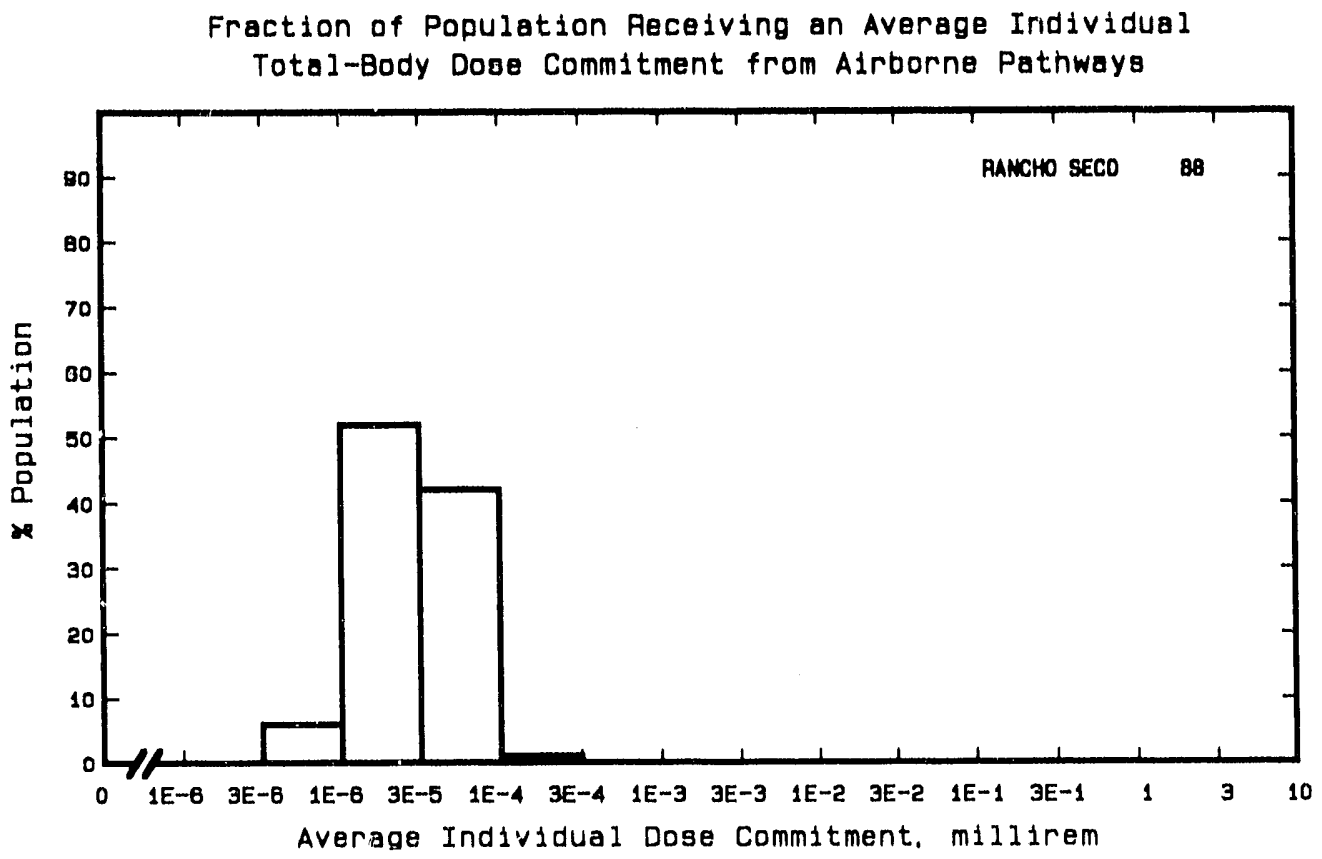


Site: RIVER BEND

ST. FRANCISVILLE, LOUISIANA

Location: $\quad$ N $30.7572^{\circ} \quad$ W $91.3317^{\circ}$

POPULATION DATA

Total Population Within 2-to-80-km Region: 7.6E5

Major Metropolitan Centers Within Region:

Center

Population

Location

Baton Rouge

240,000

$38 \mathrm{~km} \quad \mathrm{SSE}$

Denham Springs

9,000

$39 \mathrm{~km} \quad \mathrm{SE}$

SITE-SPECIFIC DATA - AIRBORNE PATHWAYS

Average Annual State Production

of Crops and Animal Products

In $80-\mathrm{km}$ Radius Circle

Regional Productivity Factor:

Animal Grazing Factor:
Veg: $1.8 \mathrm{E} 6$ kilogram

Milk: $7.8 \mathrm{E} 7$ liter

Meat: $6.1 \mathrm{E} 7$ kilogram

0.9

0.75

Meteorology Period of Record: 17 MAR 77 - 16 MAR 79 Recovery: 99\%

Average River Flow at Site: $420,000 \mathrm{ft}^{3} / \mathrm{s}$

Drinking Water: None

Fish:

Edible Harvest: $9.1^{(a)} \mathrm{kg} / \mathrm{yr}$

Dilution Factor: 1.0

(a) Individual consumption rate as given in FES (Includes shellfish). 


\section{POPULATION DOSE-COMMITMENT ESTIMATES AND \\ AVERAGE INDIVIDUAL DOSE-COMMITMENT HISTOGRAM FOR \\ RIVER BEND}

Dose Commitments (person-rem) from Waterborne Pathways

Total Body GI-LII Thyroid Bone Liver

$\begin{array}{llllll}\text { Infant } & 0.0 \mathrm{E}+00 & 0.0 \mathrm{E}+00 & 0.0 \mathrm{E}+00 & 0.0 \mathrm{E}+00 & 0.0 \mathrm{E}+00 \\ \text { Child } & 1.2 \mathrm{E}-04 & 1.4 \mathrm{E}-04 & 1.5 \mathrm{E}-06 & 6.2 \mathrm{E}-05 & 2.0 \mathrm{E}-04 \\ \text { Teen } & 8.3 \mathrm{E}-05 & 3.2 \mathrm{E}-04 & 1.3 \mathrm{E}-06 & 4.2 \mathrm{E}-05 & 1.9 \mathrm{E}-04 \\ \text { Adult } & 5.0 \mathrm{E}-04 & 2.9 \mathrm{E}-03 & 1.0 \mathrm{E}-05 & 2.8 \mathrm{E}-04 & 1.2 \mathrm{E}-03 \\ \text { TOTAL } & 7.0 \mathrm{E}-04 & 3.3 \mathrm{E}-03 & 1.3 \mathrm{E}-05 & 3.8 \mathrm{E}-04 & 1.5 \mathrm{E}-03\end{array}$

Dose Commitments (person-rem) from Airborne Pathways

\begin{tabular}{lcccccc} 
& Total Body & GI-LII & Thyroid & Bone & Liver & Lung \\
\cline { 6 - 7 } & & & & & & \\
Infant & $1.4 \mathrm{E}-05$ & $1.4 \mathrm{E}-05$ & $2.7 \mathrm{E}-04$ & $4.6 \mathrm{E}-06$ & $1.4 \mathrm{E}-05$ & $1.5 \mathrm{E}-05$ \\
Child & $1.8 \mathrm{E}-04$ & $1.8 \mathrm{E}-04$ & $1.7 \mathrm{E}-03$ & $4.8 \mathrm{E}-05$ & $1.8 \mathrm{E}-04$ & $1.9 \mathrm{E}-04$ \\
Teen & $1.2 \mathrm{E}-04$ & $1.2 \mathrm{E}-04$ & $8.0 \mathrm{E}-04$ & $3.2 \mathrm{E}-05$ & $1.2 \mathrm{E}-04$ & $1.4 \mathrm{E}-04$ \\
Adu1t & $7.1 \mathrm{E}-04$ & $7.2 \mathrm{E}-04$ & $3.2 \mathrm{E}-03$ & $1.9 \mathrm{E}-04$ & $7.2 \mathrm{E}-04$ & $7.8 \mathrm{E}-04$ \\
708AL & $1.0 \mathrm{E}-03$ & $1.0 \mathrm{E}-03$ & $6.0 \mathrm{E}-03$ & $2.7 \mathrm{E}-04$ & $1.0 \mathrm{E}-03$ & $1.1 \mathrm{E}-03$
\end{tabular}

Production/Consumption factors:

Produce: 0.011 Milk: 0.71 Meat: 0.91

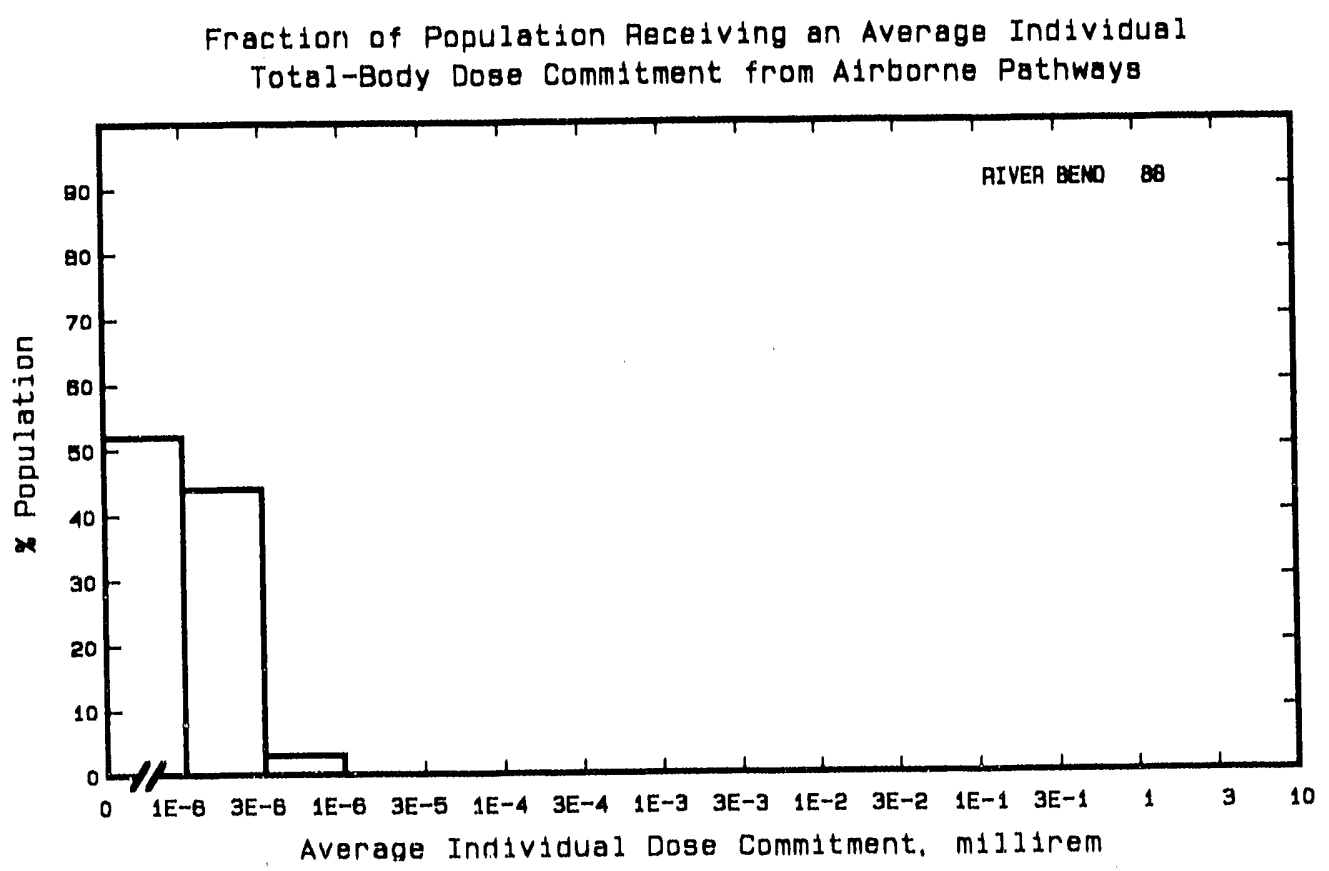


Site: H. B. ROBINSON

HARTSVILLE, SOUTH CAROLINA

Location: $\quad$ N $34.4858^{\circ} \quad$ W $80.1586^{\circ}$

POPULATION DATA

Total Population Within 2-to-80-km Region: 7.3E5

Major Metropolitan Centers Within Region:

Center

Florence

Sumter

Monroe

Lancaster
Population

32,000

27,000

14,000

10,000
Location

$42 \mathrm{~km} \quad \mathrm{ESE}$

$56 \mathrm{~km} \quad \mathrm{SSW}$

$74 \mathrm{~km} \quad \mathrm{NNW}$

$66 \mathrm{~km} \quad$ WNW

\section{SITE-SPECIFIC DATA - AIRBORNE PATHWAYS}

Average Annual State Production

of Crops and Animal Products

In $80-\mathrm{km}$ Radius Circle

Regional Productivity Factor:

Animal Grazing Factor:
Veg: 7.4E6 kilogram

Milk: 5.7E7 liter

Meat: 5.0E7 kilogram

1

0.8

Meteorology Period of Record: 1 JAN 75 - 31 DEC 75 Recovery: 94\%

\section{SITE-SPECIFIC DATA - WATERBORNE PATHWAYS via LAKE ROBINSON}

Average Dilution Flow

from Plant: $940 \mathrm{ft}^{3} / \mathrm{s}$

Drinking Water:

None

Fish:

Edible Harvest: $1.8(\mathrm{a}) \mathrm{kg} / \mathrm{yr}$

Dilution Factor: $0.02(\mathrm{~b})$

(a) Average individual consumption rate as given in the FES (1975) used in lieu of catch data.

(b) Ten percent of population obtain fish taken from water diluted by a factor of 0.2 according to FES. 
POPULATION DOSE-COMMITMENT ESTIMATES AND

AVERAGE INDIVIDUAL DOSE-COMAITMENT HISTOGRAM FOR

H. B. ROBINSON 2

Dose Commitments (person-rem) from Waterborne Pathways

Total Body GI-LII Thyroid Bone Liver

$\begin{array}{llllll}\text { Infant } & 0.0 \mathrm{E}+00 & 0.0 \mathrm{E}+00 & 0.0 \mathrm{E}+00 & 0.0 \mathrm{E}+00 & 0.0 \mathrm{E}+00 \\ \text { Child } & 2.2 \mathrm{E}-03 & 1.7 \mathrm{E}-03 & 2.2 \mathrm{E}-04 & 9.2 \mathrm{E}-03 & 9.9 \mathrm{E}-03 \\ \text { Teen } & 3.4 \mathrm{E}-03 & 3.4 \mathrm{E}-03 & 1.9 \mathrm{E}-04 & 5.5 \mathrm{E}-03 & 8.3 \mathrm{E}-03 \\ \text { Aduit } & 3.5 \mathrm{E}-02 & 2.9 \mathrm{E}-02 & 1.5 \mathrm{E}-03 & 3.2 \mathrm{E}-02 & 5.0 \mathrm{E}-02 \\ \text { TOTAL } & 4.1 \mathrm{E}-02 & 3.4 \mathrm{E}-02 & 1.9 \mathrm{E}-03 & 4.6 \mathrm{E}-02 & 6.8 \mathrm{E}-02\end{array}$

Dose Commitments (person-rem) from Airborne Pathways

Total Body GI-LII Thyroid Bone Liver Lung

$\begin{array}{lllllll}\text { Infant } & 2.8 \mathrm{E}-04 & 2.8 \mathrm{E}-04 & 7.3 \mathrm{E}-04 & 2.6 \mathrm{E}-04 & 2.8 \mathrm{E}-04 & 3.0 \mathrm{E}-04 \\ \text { Child } & 3.2 \mathrm{E}-03 & 3.2 \mathrm{E}-03 & 5.7 \mathrm{E}-03 & 2.9 \mathrm{E}-03 & 3.2 \mathrm{E}-03 & 3.5 \mathrm{E}-03 \\ \text { Teen } & 2.3 \mathrm{E}-03 & 2.3 \mathrm{E}-03 & 3.4 \mathrm{E}-03 & 2.1 \mathrm{E}-03 & 2.3 \mathrm{E}-03 & 2.7 \mathrm{E}-03 \\ \text { Adult } & 1.4 \mathrm{E}-02 & 1.4 \mathrm{E}-02 & 1.8 \mathrm{E}-02 & 1.3 \mathrm{E}-02 & 1.4 \mathrm{E}-02 & 1.5 \mathrm{E}-02 \\ \text { TOTAL } & 2.0 \mathrm{E}-02 & 2.0 \mathrm{E}-02 & 2.7 \mathrm{E}-02 & 1.8 \mathrm{E}-02 & 2.0 \mathrm{E}-02 & 2.2 \mathrm{E}-02\end{array}$

Production/Consumption factors:

Produce: $0.052 \quad$ Mi $7 k: 0.60$ Meat: 0.85

Fraction of Population Receiving an Average Individual

Total-Body Dose Commitment from Alrborne Pathways

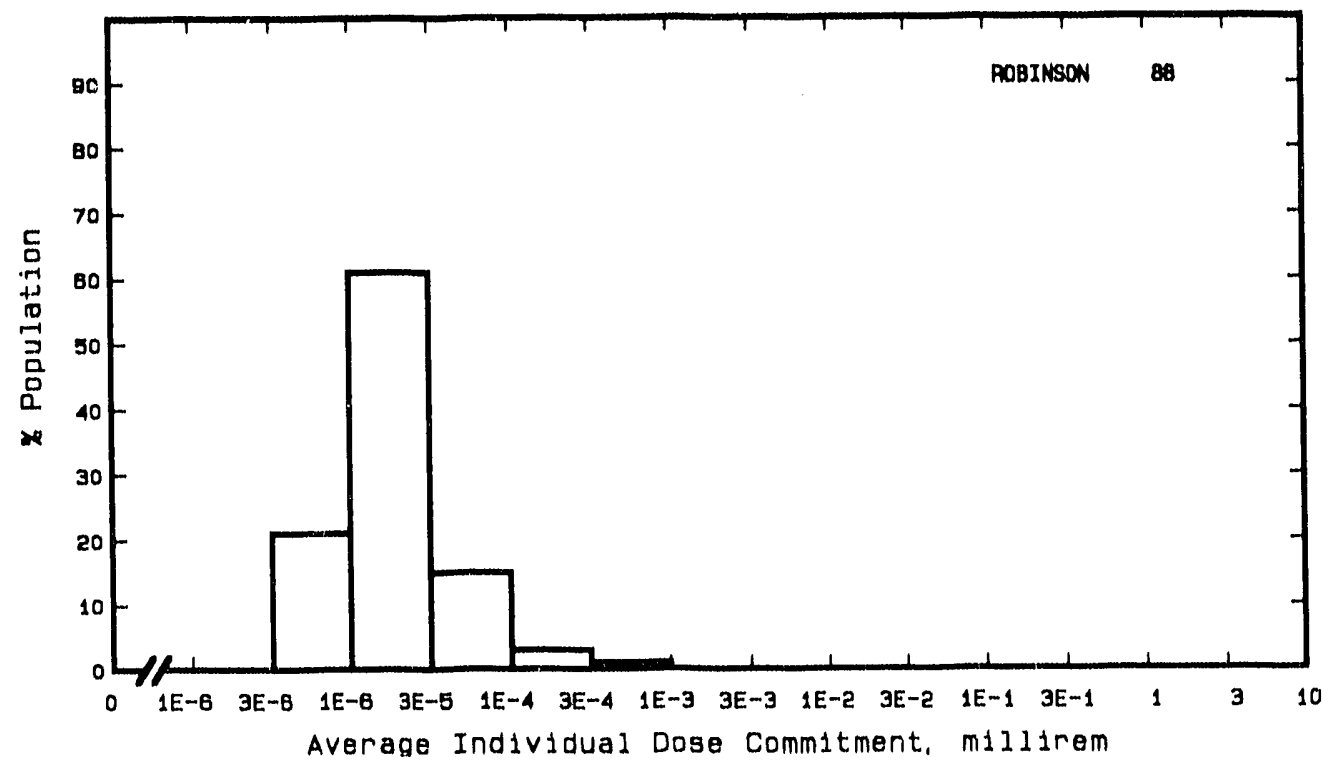


Site: ST. LUCIE

FORT PIERCE, FLORIDA

Location: $\quad$ N $27.3486^{\circ} \quad W 80.2464^{\circ}$

POPULATION DATA

Total Population Within 2-to-80-km Region: 7.2E5

Major Metropolitan Centers Within Region:

\begin{tabular}{lccc}
\multicolumn{1}{c}{ Center } & Population & \multicolumn{2}{c}{ Location } \\
\cline { 2 - 5 } & & & \\
West Palm Beach & 80,000 & $73 \mathrm{~km}$ & SSE \\
Ft. Pierce & 43,000 & $14 \mathrm{~km}$ & NW \\
Riviera Beach & 33,000 & $65 \mathrm{~km}$ & SSE \\
Vero Beach & 22,000 & $36 \mathrm{~km}$ & NNW \\
Palm Beach & 13,000 & $72 \mathrm{~km}$ & SSE
\end{tabular}

SITE-SPECIFIC DATA - AIRBORNE PATHWAYS

Average Annual State Production

of Crops and Animal Products

In $80-\mathrm{km}$ Radius Circle

Regional Productivity Factor:

Animal Grazing Factor:

Meteorology Period of Record: 1 JAN 76 - 31 DEC 76 Recovery: 92\%
Veg: $2.8 \mathrm{E} 7 \mathrm{kilogram}$

Milk: 1.1E8 1iter

Meat: $7.2 E 7$ kilogram

0.5

1

SITE-SPECIFIC DATA - WATERBORNE PATHWAYS via ATLANTIC OCEAN

Average Dilution Flow

from Plants: $4,300 \mathrm{ft}^{3} / \mathrm{s}$

Fish:

Edible Harvest: $2.6 \mathrm{E} 5 \mathrm{~kg} / \mathrm{yr}$

Invertebrates:

Edible Harvest: $2.7 E 4 \mathrm{~kg} / \mathrm{yr}$

Dilution Factor: $0.005(a)$

(a) Dilution factors as given in FES (1973). 


\section{POPULATION DOSE-COMMITMENT ESTIMATES AND \\ AVERAGE INDIVIDUAL DOSE-COMMITMENT HISTOGRAM FOR}

SAINT LUCIE 1 AND?

Dose Commitments (person-rem) from Waterborne Pathways

Total Body GI-LII Ihyroid Bone Liver

$\begin{array}{llllll}\text { Infant } & 0.0 \mathrm{E}+00 & 0.0 \mathrm{E}+00 & 0.0 \mathrm{E}+00 & 0.0 \mathrm{E}+00 & 0.0 \mathrm{E}+00 \\ \text { Child } & 2.8 \mathrm{E}-05 & 1.7 \mathrm{E}-04 & 1.7 \mathrm{E}-05 & 9.6 \mathrm{E}-05 & 6.5 \mathrm{E}-05 \\ \text { Teen } & 2.2 \mathrm{E}-05 & 3.5 \mathrm{E}-04 & 1.3 \mathrm{E}-05 & 5.5 \mathrm{E}-05 & 5.1 \mathrm{E}-05 \\ \text { Adult } & 1.6 \mathrm{E}-04 & 3.0 \mathrm{E}-03 & 8.6 \mathrm{E}-05 & 3.2 \mathrm{E}-04 & 3.0 \mathrm{E}-04 \\ \text { TOTAL } & 2.1 \mathrm{E}-04 & 3.5 \mathrm{E}-03 & 1.2 \mathrm{E}-04 & 4.7 \mathrm{E}-04 & 4.2 \mathrm{E}-04\end{array}$

Dose Commitmerts (person-rem) from Airborne Pathways

Iotal Body GI-LII Ihyroid Bone Liver Lung

$\begin{array}{lllllll}\text { Infant } & 4.9 \mathrm{E}-03 & 4.9 \mathrm{E}-03 & 2.1 \mathrm{E}-02 & 4.3 \mathrm{E}-03 & 4.9 \mathrm{E}-03 & 5.1 \mathrm{E}-03 \\ \text { Child } & 5.7 \mathrm{E}-02 & 5.7 \mathrm{E}-02 & 1.5 \mathrm{E}-01 & 4.8 \mathrm{E}-02 & 5.8 \mathrm{E}-02 & 6.1 \mathrm{E}-02 \\ \text { Teen } & 4.2 \mathrm{E}-02 & 4.2 \mathrm{E}-02 & 8.1 \mathrm{E}-02 & 3.5 \mathrm{E}-02 & 4.2 \mathrm{E}-02 & 4.7 \mathrm{E}-02 \\ \text { Adult } & 2.5 \mathrm{E}-01 & 2.5 \mathrm{E}-01 & 3.9 \mathrm{E}-01 & 2.1 \mathrm{E}-01 & 2.5 \mathrm{E}-01 & 2.7 \mathrm{E}-01 \\ \text { TOTAL } & 3.6 \mathrm{E}-01 & 3.6 \mathrm{E}-01 & 6.4 \mathrm{E}-01 & 3.0 \mathrm{E}-01 & 3.6 \mathrm{E}-01 & 3.8 \mathrm{E}-01\end{array}$

Production/Consumption factors:

Produce: $0.10 \quad$ Milk: 0.60 Meat: 0.63

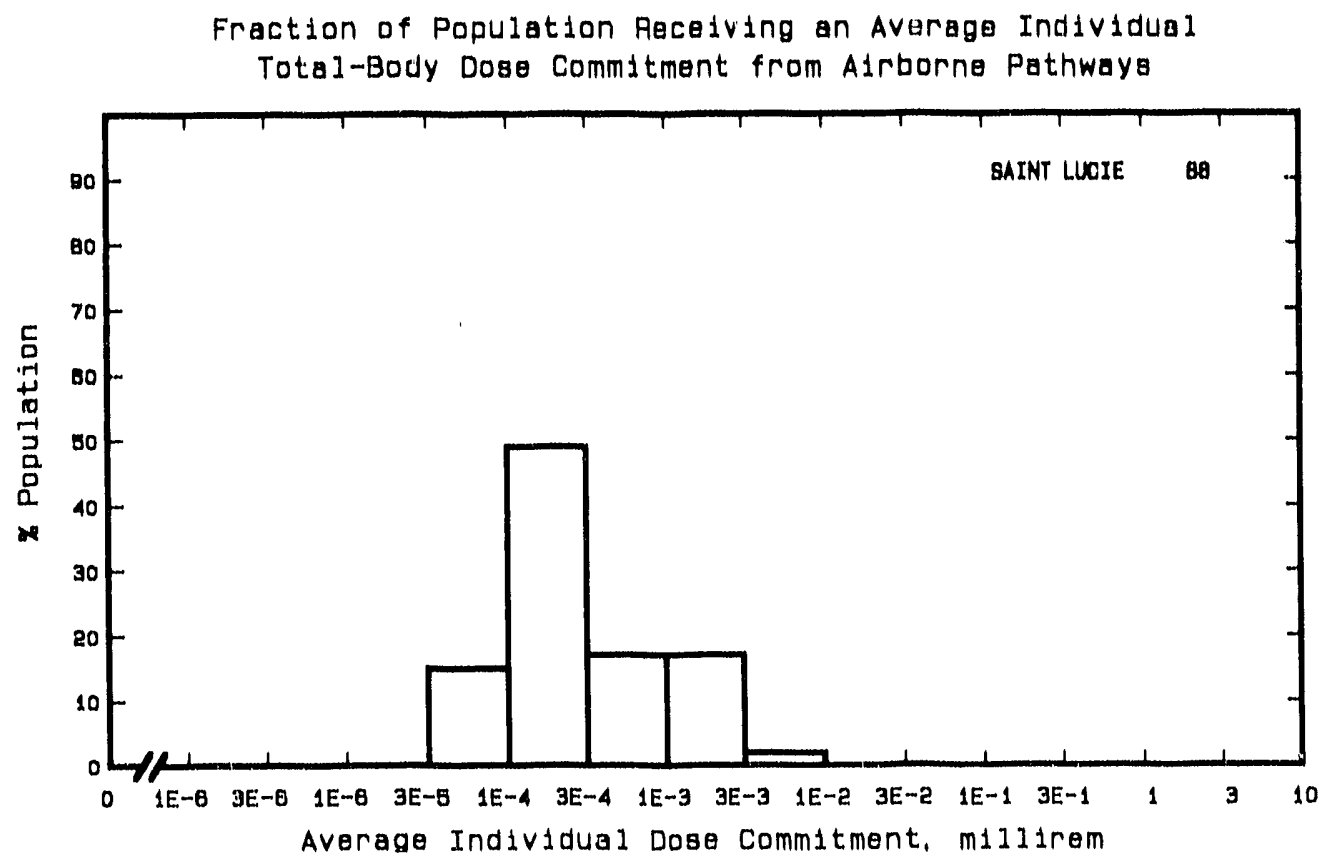


Site: SALEM

SALEM, NEW JERSEY

Location: $\quad N 39.4628^{\circ}$ W $75.5358^{\circ}$

POPULATION DATA

Total Population Within 2-to-80-km Regton: 4.9E6

Major Metropolitan Centers Within Region:

Center

Phtladelphia SMSA

Wilmington SMSA

Vineland-Millville-Bridgetion SMSA

Chester

Norristown
Population

$4,900,000$

420,000

130,000

48,000

36,000
Location

$63 \mathrm{~km} \quad \mathrm{NNE}$

$32 \mathrm{~km} \quad \mathrm{NNW}$

$48 \mathrm{~km} \quad \mathrm{E}$

$45 \mathrm{~km} \quad \mathrm{NNE}$

$74 \mathrm{~km} \mathrm{~N}$

SITE-SPECIFIC DATA - AIRBORNE PATHWAYS

Average Annual State Production

Of Crops and Animal Products

In $80-\mathrm{km}$ Radius Circle

Regional Productivity Factor:

Animal Grazing Factor:

Meteorology Period of Record: 1 JUN 70 - 31 MAY 71 Recovery: 95\%
Veg: $7.4 \mathrm{E} 7 \mathrm{k}+1$ logram

Milk: 2.7E8 liter

Meat: $2.4 E 7$ kilogram

0.9

0.6

SITE-SPECIFIC DATA - WATERBORNE PATHWAYS via DELAWARE RIVER ESTUARY

Fish:

Invertebrates: (a)
Average River Flow at Site: $16,000 \mathrm{ft}^{3} / \mathrm{s}$

Edible Harvest: $3.6 E 5 \mathrm{~kg} / \mathrm{yr}$ Dilution Factor: 1

Edible Harvest: $1.6 \mathrm{E} 5 \mathrm{~kg} / \mathrm{yr}$ Dilution Factor: 1

(a) Environment primarlly salt water so invertebrates considered in lieu of drinking water. 
POPULATION DOSE-COMMITMENT ESTIIMATES AND

AVERAGE INDIVIDUAL DOSE-COMMITMENT HISTOGRAM FOR

\section{SALEM 1 AND 2}

Dose Commitments (person-rem) from Waterborne Pathways

Total Body

$$
\text { GI-LII }
$$

Ihyrotd

Bone

Liver

Infant

$0.0 E+00$

$0.0 \mathrm{E}+00$

$0.0 E+00$

$0.0 \mathrm{E}+00$

$0.0 E+00$

Child

$4.5 \mathrm{E}-02$

7. $0 \mathrm{E}-02$

2. $9 \mathrm{E}-02$

1. $5 \mathrm{E}-01$

9.3E-02

Teen

3.1E-02

$1.4 \mathrm{E}-01$

2. $0 \mathrm{E}-01$

$1.2 \mathrm{E}+00$

2.1E-02

8.1E-02

7.2E -02

Adult

2. $8 \mathrm{E}-01$

$1.5 \mathrm{E}+00$

$1.4 \mathrm{E}-01$

$4.8 \mathrm{E}-01$

$4.2 E-01$

TOTAL

Dose Commitments (person-rem) from Airborne Pathways

Total Body GI-LII Thyrotd Bone Liver Lung

$\begin{array}{llllllll}\text { Infant } & 8.3 \mathrm{E}-03 & 8.3 \mathrm{E}-03 & 1.0 \mathrm{E}-02 & 1.4 \mathrm{E}-03 & 8.3 \mathrm{E}-03 & 8.4 \mathrm{E}-03 \\ \text { Child } & 1.2 \mathrm{E}-01 & 1.2 \mathrm{E}-01 & 1.3 \mathrm{E}-01 & 1.6 \mathrm{E}-02 & 1.2 \mathrm{E}-01 & 1.2 \mathrm{E}-01 \\ \text { Teen } & 8.1 \mathrm{E}-02 & 8.1 \mathrm{E}-02 & 8.6 \mathrm{E}-02 & 1.2 \mathrm{E}-02 & 8.1 \mathrm{E}-02 & 8.4 \mathrm{E}-02 \\ \text { Adult } & 4.5 \mathrm{E}-01 & 4.5 \mathrm{E}-01 & 4.7 \mathrm{E}-01 & 7.1 \mathrm{E}-02 & 4.5 \mathrm{E}-01 & 4.6 \mathrm{E}-01 \\ \text { TOTAL } & 6.6 \mathrm{E}-01 & 6.6 \mathrm{E}-01 & 6.9 \mathrm{E}-01 & 1.0 \mathrm{E}-01 & 6.6 \mathrm{E}-01 & 6.7 \mathrm{E}-01\end{array}$

Production/Consumption factors:

Produce: $0.070 \quad$ Milk: 0.38 Meat: 0.055

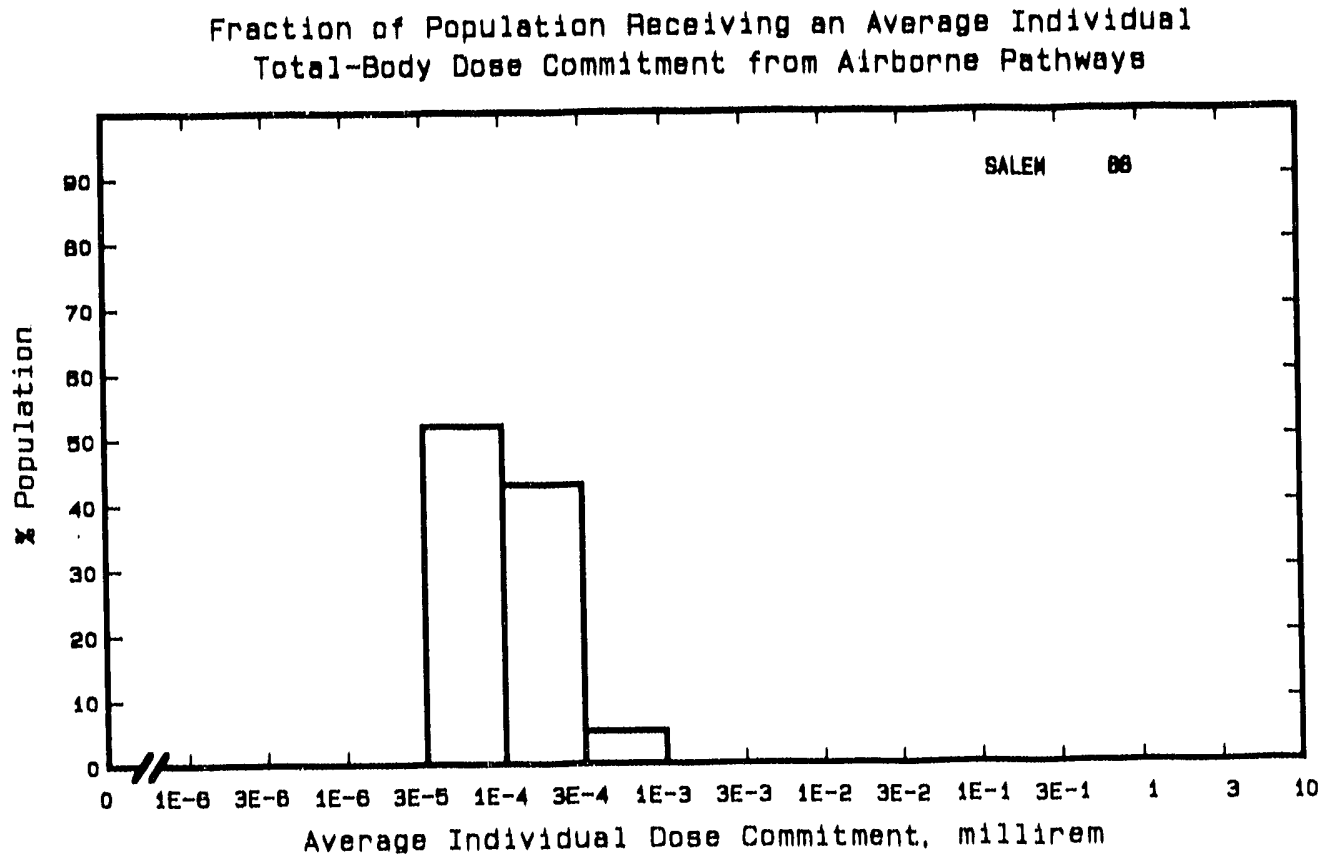


Site: SAN ONOFRE

CAMP PENDLETON, CALIFORNIA

Location: $\quad$ N $33.3703^{\circ} \quad$ W $117.5569^{\circ}$

POPULATION DATA

Total Population Within 2-to-80-km Region: 5.5E6

Major Metropolitan Centers Within Region:

Center

Population

Location

San Diego SMSA

$2,100,000$

Anaheim-Santa Ana-Garden Grove SMSA

Long Beach

Huntington Beach

$2,100,000$

410,000

180,000

Riverside

180,000

Pomona

110,000

$\begin{array}{lll}68 & \mathrm{~km} & \text { SSE } \\ 62 & \mathrm{~km} & \mathrm{NW} \\ 75 & \mathrm{~km} & \mathrm{NW} \\ 61 & \mathrm{~km} & \mathrm{NW} \\ 68 & \mathrm{~km} & \mathrm{~N} \\ 79 \mathrm{~km} & \mathrm{NNW}\end{array}$

SITE-SPECIFIC DATA - AIRBORNE PATHWAYS

Average Annual State Production

of Crops and Animal Products

In $80-\mathrm{km}$ Radilis Circle

Veg: $4.8 E 7$ kilogram

Milk: 2.3E8 1iter

Meat: $5.0 E 7$ kilogram

Regional Productivity Factor:

Animal Grazing Factor:

0.6

1

Meteorology Period of Record: 25 JAN 73 - 24 JAN 76 Recovery: 88\%

SITE-SPECIFIC DATA - WATERBORNE PATHWAYS VIa PACIFIC OCEAN

Average Dilution Flow

from all 3 Plants: $3,500 \mathrm{ft}^{3} / \mathrm{s}$

Fish:

Edible Harvest: $2.9 E 4 \mathrm{~kg} / \mathrm{yr}$

Dilution Factor: 1 (a)

Invertebrates:

Edible Harvest: $2.9 \mathrm{E} 3 \mathrm{~kg} / \mathrm{yr}$

Dilution Factor: i $(a)$

(a) Seafood caught in undlluted effluent according to FES (1973). 
POPULATION DOSE-COMMITMENT ESTIMATES AND

AVERAGE INDIVIDUAL DOSE-COMMITMENT HISTOGRAM FOR

SAN ONOFRE 1, 2, AND 3

Dose Commitments (person-rem) from Waterborne Pathways

Total Body GI-LII Thyroid Bone Liver

$\begin{array}{llllll}\text { Infant } & 0.0 \mathrm{E}+00 & 0.0 \mathrm{E}+00 & 0.0 \mathrm{E}+00 & 0.0 \mathrm{E}+00 & 0.0 \mathrm{E}+00 \\ \text { Child } & 1.5 \mathrm{E}-03 & 3.8 \mathrm{E}-03 & 1.8 \mathrm{E}-02 & 4.6 \mathrm{E}-03 & 5.2 \mathrm{E}-03 \\ \text { Teen } & 2.0 \mathrm{E}-03 & 7.7 \mathrm{E}-03 & 1.3 \mathrm{E}-02 & 2.7 \mathrm{E}-03 & 4.4 \mathrm{E}-03 \\ \text { Adult } & 1.9 \mathrm{E}-02 & 6.7 \mathrm{E}-02 & 8.3 \mathrm{E}-02 & 1.6 \mathrm{E}-02 & 2.7 \mathrm{E}-02 \\ \text { TOTAL } & 2.3 \mathrm{E}-02 & 7.9 \mathrm{E}-02 & 1.1 \mathrm{E}-01 & 2.3 \mathrm{E}-02 & 3.6 \mathrm{E}-02\end{array}$

Dose Commitments (person-rem) from Airborne Pathways

Total Body GI-LII Thyroid Bone Liver Lung

$\begin{array}{lllllll}\text { Infant } & 7.0 \mathrm{E}-03 & 6.8 \mathrm{E}-03 & 1.2 \mathrm{E}-01 & 6.9 \mathrm{E}-03 & 7.2 \mathrm{E}-03 & 7.3 \mathrm{E}-03 \\ \text { Child } & 7.8 \mathrm{E}-02 & 7.7 \mathrm{E}-02 & 6.8 \mathrm{E}-01 & 7.5 \mathrm{E}-02 & 7.9 \mathrm{E}-02 & 8.4 \mathrm{E}-02 \\ \text { Teen } & 5.7 \mathrm{E}-02 & 5.6 \mathrm{E}-02 & 3.0 \mathrm{E}-01 & 5.4 \mathrm{E}-02 & 5.7 \mathrm{E}-02 & 6.6 \mathrm{E}-02 \\ \text { Adult } & 3.4 \mathrm{E}-01 & 3.4 \mathrm{E}-01 & 1.2 \mathrm{E}+00 & 3.3 \mathrm{E}-01 & 3.4 \mathrm{E}-01 & 3.7 \mathrm{E}-01 \\ \text { TOTAL } & 4.8 \mathrm{E}-01 & 4.8 \mathrm{E}-01 & 2.3 \mathrm{E}+00 & 4.6 \mathrm{E}-01 & 4.9 \mathrm{E}-01 & 5.3 \mathrm{E}-01\end{array}$

Production/Consumption factors:

Produce: 0.027

Milk: 0.19

Meat: 0.067
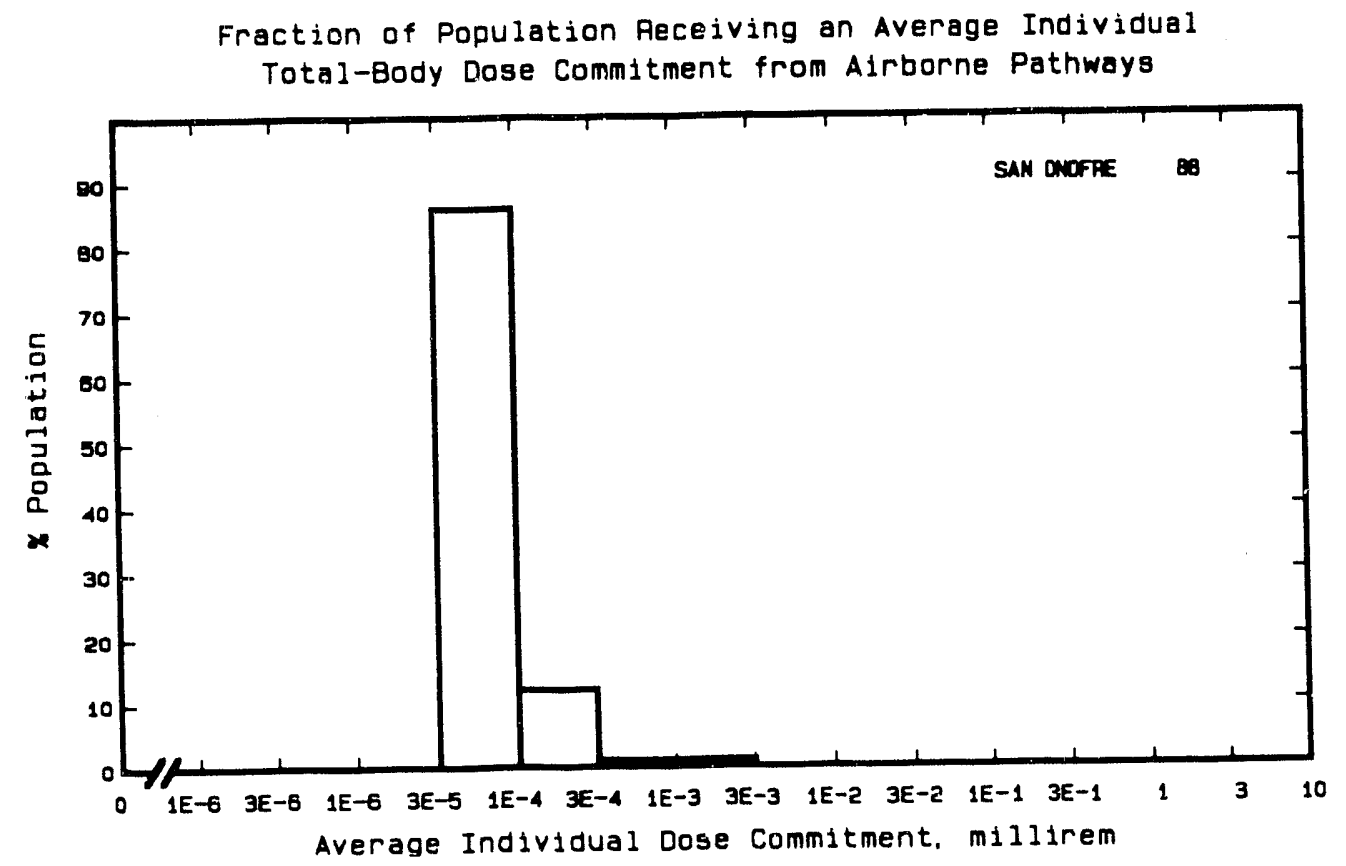
Site: SEQUOYAH

HAMILTON COUNTY, TENNESSEE

Location: $\quad$ N $35.2233^{\circ} \quad$ W $85.0878^{\circ}$

POPULATION DATA

Total Population Within 2-to-80-km Region: 8.8E5

Major Metropolitan Centers Within Region:

Center

Chattanooga SMSA

Clevel and

East Ridge

Dalton

Athens
Population

440,000

26,000

21,000

21,000

12,000
Location

$28 \mathrm{~km} \quad \mathrm{SW}$

$21 \mathrm{~km} \quad \mathrm{SE}$

$27 \mathrm{~km} \quad \mathrm{SSW}$

$50 \mathrm{~km} \mathrm{~S}$

$53 \mathrm{~km} \quad$ ENE

SITE-SPECIFIC DATA - AIRBORNE PATHWAYS

Average Annual State Production

of Crops and Animal Products

In $80-\mathrm{km}$ Radius Circle

Regional Productivity Factor:

Animal Grazing Factor:
Veg: $1.1 \mathrm{E7}$ kilogram

Milk: $1.6 \mathrm{E} 8$ liter

Meat: 1.2E8 kilogram

0.25

0.7

Meteorology Period of Record: 1 JAN 72 - 31 DEC 72 Recovery: 93\%

SITE-SPECIFIC DATA - WATERBORNE PATHWAYS via TENNESSEE RIVER

Drinking Water:

Average River Flow

at Site: $35,000 \mathrm{ft}^{3} / \mathrm{s}$

Fish:

Exposed Population: 460,000

Dilution Factor: 1

Edible Harvest: $1.7 \mathrm{E}^{(\mathrm{a})} \mathrm{kg} / \mathrm{yr}$

Dilution Factor: 1

(a) Catch data given in FES (1974). 


\section{POPULATION DOSE-COMMITMENT ESTIMATES AND \\ AVERAGE INDIVIDUAL DOSE-COMMITMENT HISTOGRAM FOR}

SEQUOYAH 1 AND 2

Dose Commitments (person-rem) from Waterborne Pathways

Total Body GI-LII Ihyroid Bone Liver

$\begin{array}{llllll}\text { Infant } & 2.7 \mathrm{E}-03 & 2.5 \mathrm{E}-03 & 3.0 \mathrm{E}-03 & 2.1 \mathrm{E}-03 & 4.7 \mathrm{E}-03 \\ \text { Child } & 3.5 \mathrm{E}-02 & 3.0 \mathrm{E}-02 & 3.0 \mathrm{E}-02 & 4.4 \mathrm{E}-02 & 6.9 \mathrm{E}-02 \\ \text { Teen } & 2.1 \mathrm{E}-02 & 1.4 \mathrm{E}-02 & 1.1 \mathrm{E}-02 & 1.8 \mathrm{E}-02 & 3.6 \mathrm{E}-02 \\ \text { Adult } & 2.0 \mathrm{E}-01 & 1.3 \mathrm{E}-01 & 9.2 \mathrm{E}-02 & 1.1 \mathrm{E}-01 & 2.4 \mathrm{E}-01 \\ \text { TOTAL } & 2.6 \mathrm{E}-01 & 1.7 \mathrm{E}-01 & 1.4 \mathrm{E}-01 & 1.7 \mathrm{E}-01 & 3.5 \mathrm{E}-01\end{array}$

Dose Commitments (person-rem) from Airborne Pathways

Total Body GI-LII Thyroid Bone Liver

$\begin{array}{lllllll}\text { Infant } & 2.4 \mathrm{E}-04 & 2.4 \mathrm{E}-04 & 2.5 \mathrm{E}-04 & 1.6 \mathrm{E}-04 & 2.4 \mathrm{E}-04 & 2.6 \mathrm{E}-04 \\ \text { Child } & 3.1 \mathrm{E}-03 & 3.1 \mathrm{E}-03 & 3.1 \mathrm{E}-03 & 1.8 \mathrm{E}-03 & 3.1 \mathrm{E}-03 & 3.3 \mathrm{E}-03 \\ \text { Teen } & 2.3 \mathrm{E}-03 & 2.3 \mathrm{E}-03 & 2.3 \mathrm{E}-03 & 1.3 \mathrm{E}-03 & 2.3 \mathrm{E}-03 & 2.6 \mathrm{E}-03 \\ \text { Adult } & 1.4 \mathrm{E}-02 & 1.4 \mathrm{E}-02 & 1.4 \mathrm{E}-02 & 8.0 \mathrm{E}-03 & 1.4 \mathrm{E}-02 & 1.5 \mathrm{E}-02 \\ \text { TOTAL } & 1.9 \mathrm{E}-02 & 1.9 \mathrm{E}-02 & 2.02 \mathrm{E}-02 & 1.1 \mathrm{E}-02 & 1.9 \mathrm{E}-02 & 2.1 \mathrm{E}-02\end{array}$

Production/Consumption factors:

Produce: $0.016 \quad$ Milk: 0.36 Meat: 0.42

Fraction of Population Receiving an Average Individual

Tota1-Body Dose Commitment from Alrborne Pathways

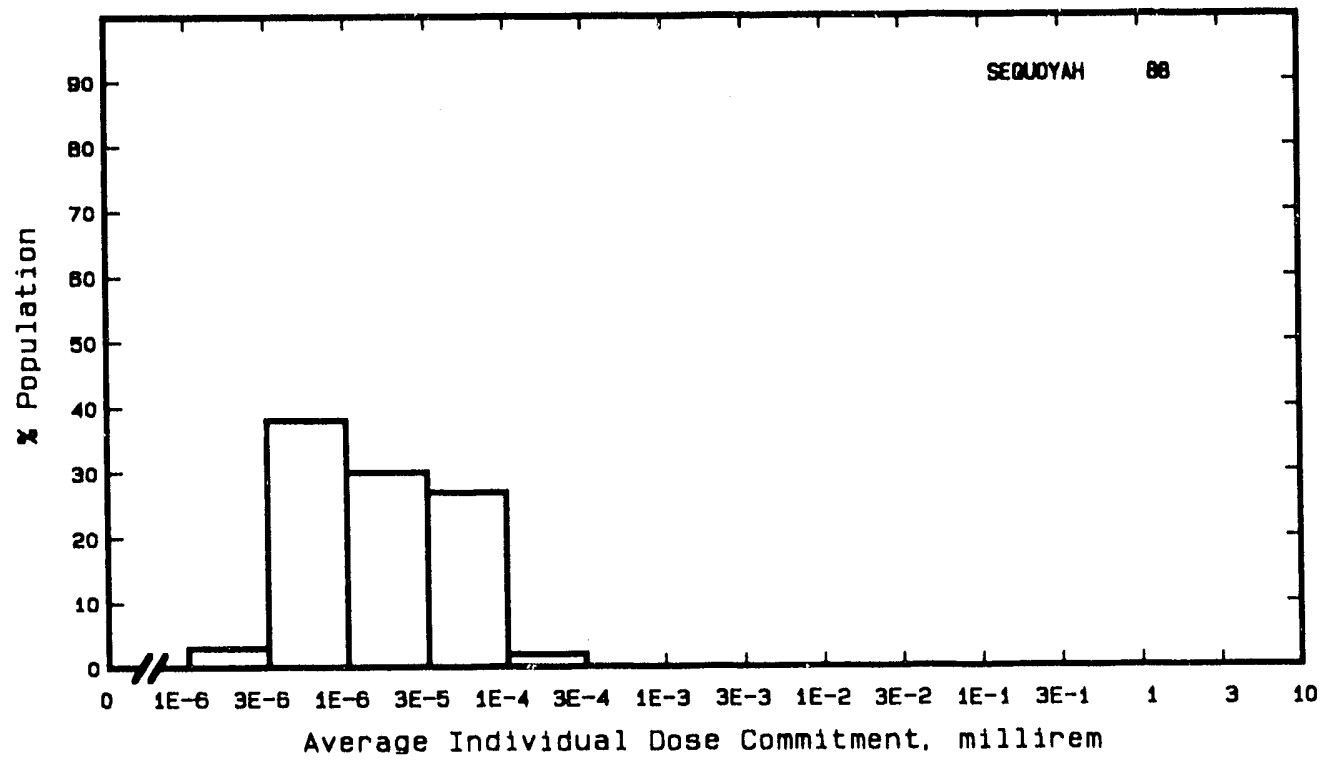


Site: SHOREHAM

BROOKHAVEN, NEW YORK

Location: $\quad$ N $40.9608^{\circ} \quad$ W $72.8658^{\circ}$

POPULATION DATA

Total Population Within 2-to-80-km Region: 5.2E6

Major Metropolitan Centers Within Region:

Center

Nassau county - Suffolk County SMSA

New Haven - West Haven SMSA

Bridgeport SMSA

Waterbury SMSA

Stamford SMSA

Norwalk

Meriden
Population

$2,600,000$

430,000

400,000

230,000

130,000

80,000

59,000
Location

$50 \mathrm{~km} \quad W S W$

$38 \mathrm{~km} \quad \mathrm{NW}$

$37 \mathrm{~km} \quad \mathrm{NW}$

$68 \mathrm{~km} \quad \mathrm{NNW}$

$57 \mathrm{~km} \quad W N W$

$26 \mathrm{~km} \quad W N W$

$68 \mathrm{~km} \mathrm{~N}$

SITE-SPECIFIC DATA - AIRBORNE PATHWAYS

Average Annual State Production

of Crops and Animal Products

In $80-\mathrm{km}$ Radius Circle

Regional Productivity Factor:

Animal Grazing Factor:

Meteorology Period of Record: 1 OCT 73 - 30 SEP 74 Recovery: $90 \%$
Veg: $7.6 \mathrm{E} 7 \mathrm{kilogram}$

Milk: 7.0E8 liter

Meat: $3.3 \mathrm{E} 7 \mathrm{ki}$ Iogram

0.5

0.6

SITE-SPECIFIC DATA - WATERBORNE PATHWAYS via LONG ISLAND SOUND

Average Dilution Flow

from Plant: $1.0 \mathrm{ft}^{3} / \mathrm{s}$

Fish:

Edible Harvest: $2.9 E 5 \mathrm{~kg} / \mathrm{yr}$

Dilution Factor: 0.001

Invertebrates:

Edible Harvest: $3.6 \mathrm{E} 5 \mathrm{~kg} / \mathrm{yr}$

Dilution Factor: 0.1 


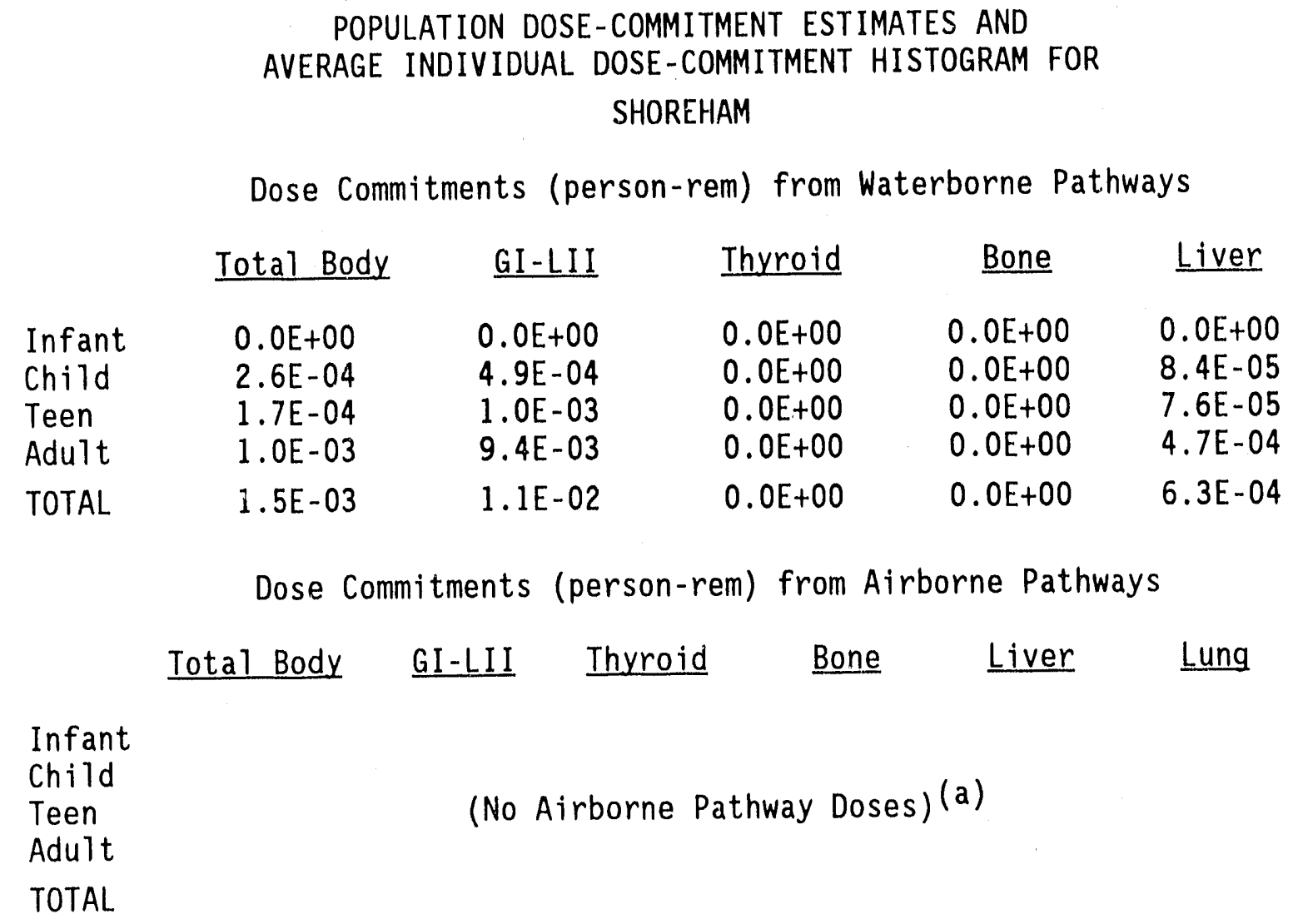

(a) No atmospheric re leases reported (Tichler, et al. 1991). 
Site: SOUTH TEXAS

PALACIOS, TEXAS
Location:
$N 28.7000^{\circ}$
W $96.2133^{\circ}$

POPULATION DATA

Total Population Within 2-to-80-km Region: 8.9E5

Major Metropolitan Centers Within Region:

Center Population Location

$\begin{array}{llll}\text { Bay City } & 21,000 & 19 \mathrm{~km} & \text { NNE } \\ \text { Lake Jackson } & 23,000 & 67 \mathrm{~km} & \text { ENE } \\ \text { Freeport } & 16,000 & 72 \mathrm{~km} & \text { ENE } \\ \text { Angleton } & 16,000 & 74 \mathrm{~km} & \text { NE }\end{array}$

SITE-SPECIFIC DATA - AIRBORNE PATHWAYS

Average Annual State Production

of Crops and Animal Products

In $80-\mathrm{km}$ Radius Circle

Regional Productivity Factor:

Animal Grazing Factor:
Veg: $2.3 \mathrm{E} 7 \mathrm{kilogram}$

Milk: $4.3 E 7$ liter

Meat: $1.1 \mathrm{E} 8$ kilogram

0.4

0.9

Meteorology Period of Record: 1 JAN 89 - 31 DEC 89 Recovery: N/A

SITE-SPECIFIC DATA - WATERBORNE PATHWAYS via COLORADO RIVER

Average River Flow

at Site: $800 \mathrm{ft}^{3} / \mathrm{s}$

Drinking Water:

Exposed Population:

Dilution Factor: 1

Fish:

Edible Harvest: $0.18 \mathrm{~kg} / \mathrm{yr}(\mathrm{a})$

Dilution Factor: 1

Invertebrates:

Edible Harvest: $0.91 \mathrm{~kg} / \mathrm{yr}(\mathrm{b})$

Dilution Factor: 1

(a) Ten percent of population assumed to eat $5 \mathrm{~g} / \mathrm{d}$ of fish used in lieu of catch data.

(b) Average individual consumption rate of $2.5 \mathrm{~g} / \mathrm{d}$ as given in the FES (1972) used in 1 ieu of catch data. 


\section{POPULATION DOSE-COMMITMENT ESTIMATES AND}

AVERAGE INDIVIDUAL DOSE-COMMITMENT HISTOGRAM FOR

\section{SOUTH TEXAS}

Dose Commitments (person-rem) from Waterborne Pathways

Total Body GI-LII Thyroid Bone Liver

$\begin{array}{llllll}\text { Infant } & 0.0 \mathrm{E}+00 & 0.0 \mathrm{E}+00 & 0.0 \mathrm{E}+00 & 0.0 \mathrm{E}+00 & 0.0 \mathrm{E}+00 \\ \text { Child } & 2.3 \mathrm{E}-02 & 5.2 \mathrm{E}-02 & 1.8 \mathrm{E}-02 & 7.0 \mathrm{E}-03 & 1.8 \mathrm{E}-02 \\ \text { Teen } & 1.6 \mathrm{E}-02 & 1.1 \mathrm{E}-01 & 1.2 \mathrm{E}-02 & 4.2 \mathrm{E}-03 & 1.6 \mathrm{E}-02 \\ \text { Adult } & 9.7 \mathrm{E}-02 & 9.5 \mathrm{E}-01 & 8.3 \mathrm{E}-02 & 2.5 \mathrm{E}-02 & 9.7 \mathrm{E}-02 \\ \text { TOTAL } & 1.4 \mathrm{E}-01 & 1.1 \mathrm{E}+00 & 1.1 \mathrm{E}-01 & 3.6 \mathrm{E}-02 & 1.3 \mathrm{E}-01\end{array}$

Dose Commitments (person-rem) from Airborne Pathways

Total Body GI-LII Thyroid Bone Liver Lung

$\begin{array}{lllllll}\text { Infant } & 4.0 \mathrm{E}-04 & 4.0 \mathrm{E}-04 & 5.0 \mathrm{E}-04 & 3.9 \mathrm{E}-04 & 4.0 \mathrm{E}-04 & 4.0 \mathrm{E}-04 \\ \text { Child } & 4.5 \mathrm{E}-03 & 4.5 \mathrm{E}-03 & 5.1 \mathrm{E}-03 & 4.3 \mathrm{E}-03 & 4.5 \mathrm{E}-03 & 4.5 \mathrm{E}-03 \\ \text { Teen } & 3.3 \mathrm{E}-03 & 3.3 \mathrm{E}-03 & 3.5 \mathrm{E}-03 & 3.2 \mathrm{E}-03 & 3.3 \mathrm{E}-03 & 3.3 \mathrm{E}-03 \\ \text { Adu1t } & 2.0 \mathrm{E}-02 & 2.0 \mathrm{E}-02 & 2.1 \mathrm{E}-02 & 1.9 \mathrm{E}-02 & 2.0 \mathrm{E}-02 & 2.0 \mathrm{E}-02 \\ \text { TOTAL } & 2.8 \mathrm{E}-02 & 2.8 \mathrm{E}-02 & 3.0 \mathrm{E}-02 & 2.7 \mathrm{E}-02 & 2.8 \mathrm{E}-02 & 2.8 \mathrm{E}-02\end{array}$

Production/Consumption factors:

Produce: $0.17 \quad$ Milk: 0.48 Meat: 1.9
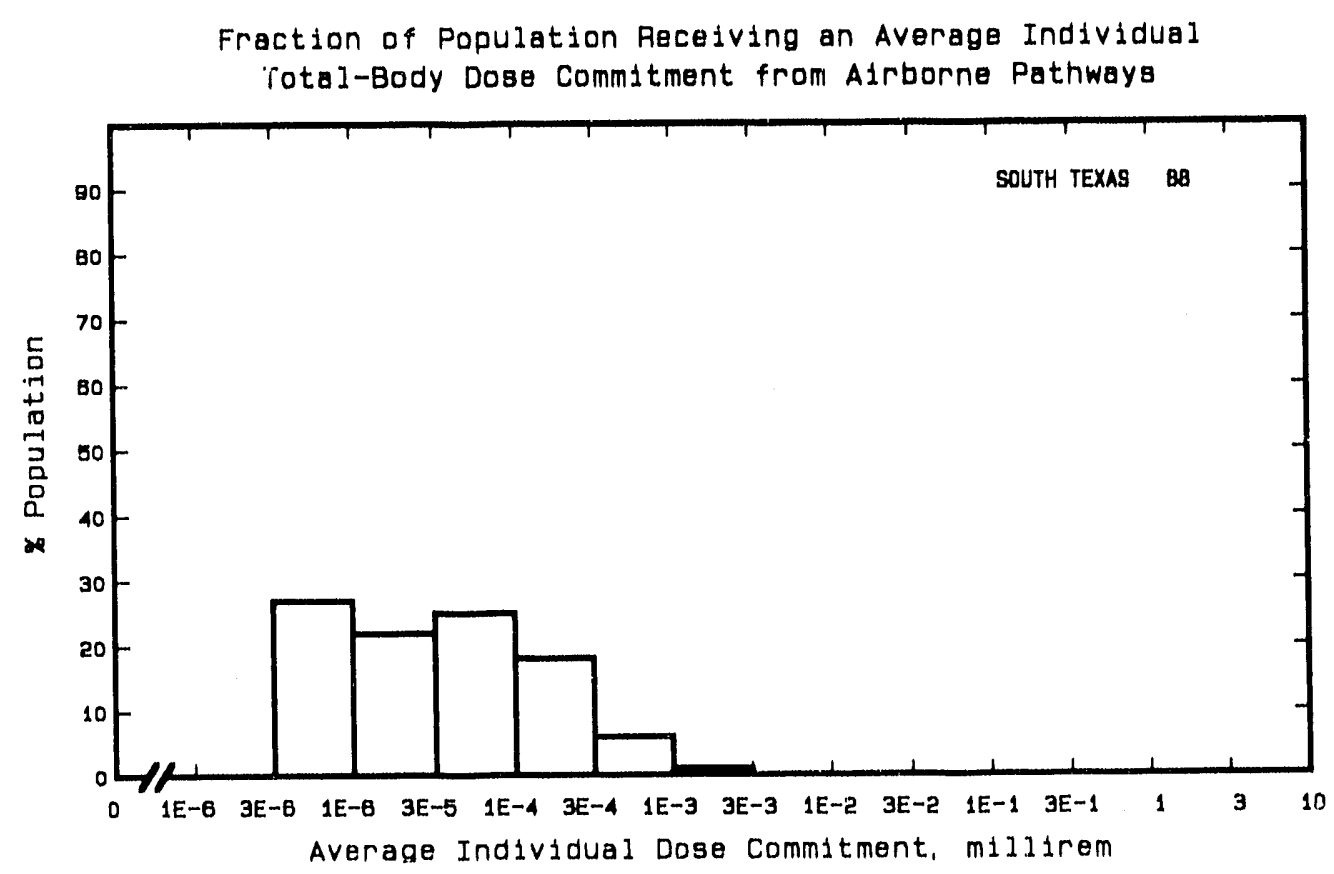
Site: SUMMER

JENKINSVILLE, SOUTH CAROLINA

Location: N $\quad$ N $34.2958^{\circ} \quad$ W $81.3203^{\circ}$

POPULATION DATA

Total Population Within 2-to-80-km Region: $9.0 E 5$

Major Metropolitan Centers Within Region:

Center

Columbia SMSA

Rock Hil1

Greenwood

Union

Laurens
Population

460,000

36,000

23,000

11,000

11,000
Location

$42 \mathrm{~km} \quad \mathrm{SE}$

$75 \mathrm{~km} \quad \mathrm{NNE}$

$78 \mathrm{~km} \mathrm{~W}$

$54 \mathrm{~km} \quad \mathrm{NNW}$

$68 \mathrm{~km} \quad W N W$

SITE-SPECIFIC DATA - AIRBORNE PATHWAYS

Average Annual State Production

of Crops and Animal Products

In $80-\mathrm{km}$ Radius Circle

Regional Productivity Factor:

Animal Grazing Factor:

Meteorology Period of Record: 1 Jan 75 - 31 Dec 75 Recovery: $98 \%$
Veg: $\quad 7.4 \mathrm{E} 6 \mathrm{kilogram}$

Milk: 5.7 E7 liter

Meat: $5.0 E 7$ kilogram

0.9

0.7

\section{-}

SITE-SPECIFIC DATA - WATERBORNE PATHWAYS via PARR RES, and BROAD RIVER

Average River Flow

at Site: $6,000 \mathrm{ft}^{3} / \mathrm{s}$

Lrinking Water: $\quad$ Exposed Population: 130,000

Dilution Factor: 1

Fish:

Edible Harvest: (a)

Dilution Factor: 1

(a) Average individual consumption rate of $2.2 \mathrm{~kg} / \mathrm{yr}$ as given in the FES (1973) used in lifeu of catch data. 
POPULATION DOSE-COMMITMENT ESTIMATES AND

AVERAGE INDIVIDUAL DOSE-COMMITMENT HISTOGRAM FOR

\section{SUMMER}

Dose Commitments (person-rem) from Waterborne Pathways

Total Body GI-LII Thyroid Bone Liver

$\begin{array}{llllll}\text { Infant } & 1.5 \mathrm{E}-02 & 1.5 \mathrm{E}-02 & 5.3 \mathrm{E}-02 & 4.8 \mathrm{E}-03 & 2.1 \mathrm{E}-02 \\ \text { Child } & 7.0 \mathrm{E}-01 & 2.7 \mathrm{E}-01 & 4.8 \mathrm{E}-01 & 2.1 \mathrm{E}+00 & 3.0 \mathrm{E}+00 \\ \text { Teen } & 1.1 \mathrm{E}+00 & 2.8 \mathrm{E}-01 & 1.8 \mathrm{E}-01 & 1.3 \mathrm{E}+00 & 2.4 \mathrm{E}+00 \\ \text { Aduit } & 1.1 \mathrm{E}+01 & 2.5 \mathrm{E}+00 & 1.3 \mathrm{E}+00 & 7.5 \mathrm{E}+00 & 1.5 \mathrm{E}+01 \\ \text { TOTAL } & 1.3 \mathrm{E}+01 & 3.0 \mathrm{E}+00 & 2.0 \mathrm{E}+00 & 1.1 \mathrm{E}+01 & 2.0 \mathrm{E}+01\end{array}$

Dose Commitments (person-rem) from Airborne Pathways

Total Body GI-LII Thyrotd Bone Liver Lung

$\begin{array}{lllllll}\text { Infant } & 1.0 \mathrm{E}-04 & 9.9 \mathrm{E}-05 & 7.3 \mathrm{E}-04 & 9.7 \mathrm{E}-05 & 1.0 \mathrm{E}-04 & 1.1 \mathrm{E}-04 \\ \text { Child } & 1.1 \mathrm{E}-03 & 1.1 \mathrm{E}-03 & 4.9 \mathrm{E}-03 & 1.1 \mathrm{E}-03 & 1.1 \mathrm{E}-03 & 1.2 \mathrm{E}-03 \\ \text { Teen } & 8.2 \mathrm{E}-04 & 8.2 \mathrm{E}-04 & 2.5 \mathrm{E}-03 & 7.8 \mathrm{E}-04 & 8.3 \mathrm{E}-04 & 9.6 \mathrm{E}-04 \\ \text { Adult } & 5.0 \mathrm{E}-03 & 5.0 \mathrm{E}-03 & 1.1 \mathrm{E}-02 & 4.7 \mathrm{E}-03 & 5.0 \mathrm{E}-03 & 5.4 \mathrm{E}-03 \\ \text { TOTAL } & 7.0 \mathrm{E}-03 & 7.0 \mathrm{E}-03 & 1.9 \mathrm{E}-02 & 6.6 \mathrm{E}-03 & 7.1 \mathrm{E}-03 & 7.7 \mathrm{E}-03\end{array}$

Production/Consumption factors:

Produce: $0.038 \quad$ Milk: $0.44 \quad$ Meat: 0.62

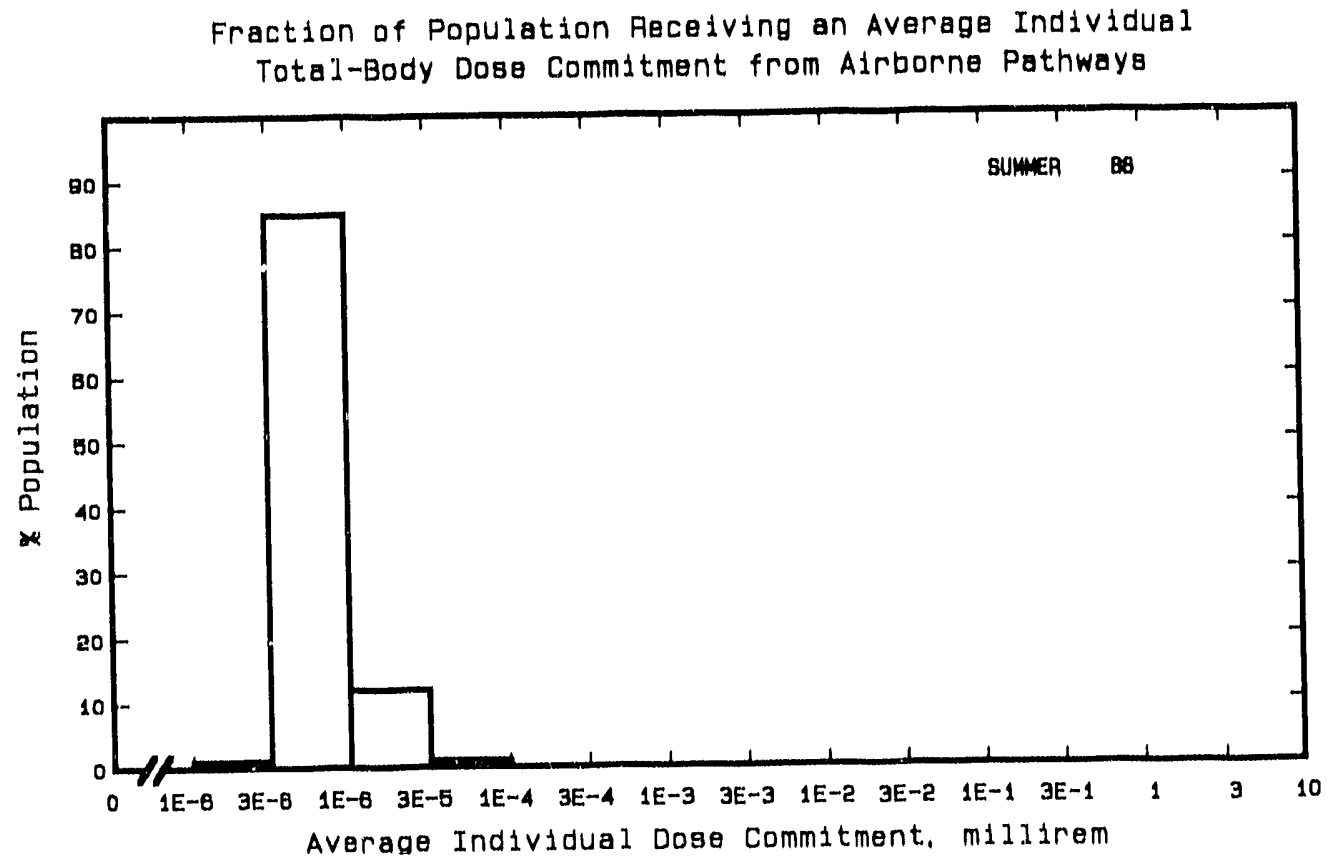


Site: SURRY

SURRY COUNTY, VIRGINIA
Location:
N $37.1656^{\circ}$
W $76.6983^{\circ}$

POPULATION DATA

Total Population Within 2-to-80-km Region: 1,9E6

Major Metropolitan Centers Within Region:

Center

Norfolk-Virginia Beach-Portsmouth SMSA

Richmond SMSA

Newport News-Hampton SMSA

Petersburg-Colonial Heights-Hopewell SMSA

Willitamsburg
Population

850,000

670,000

380,000

130,000

10,000 location

$50 \mathrm{~km} \quad \mathrm{SE}$

$77 \mathrm{~km}$ WNW

$33 \mathrm{~km}$ ESE

$63 \mathrm{~km} \mathrm{~W}$

$12 \mathrm{~km} \mathrm{~N}$

SITE-SPECIFIC DATA - AIRBORNE PATHWAYS

Average Annual State Production

of Crops and Animal Products

In $80-\mathrm{km}$ Radius Circle

Regional Productivity Factor:

Animal Grazing Factor:
Veg: $3.5 \mathrm{E} 7 \mathrm{kilogram}$

Milk: 1.5E8 Titer

Meat: $7.4 \mathrm{E} 7 \mathrm{kilogram}$

0.8

0.7

Meteorology Period of Record: 3 MAR 74 - 2 MAR 75 Recovery: $91 \%$

Average River Flow

at Site: $25,000 \mathrm{ft}^{3} / \mathrm{s}(\mathrm{a})$

Fish:

Edible Harvest: $6.055 \mathrm{~kg} / \mathrm{yr}$

Dilution Factor: 1

Invertebrates: (b)
Edible Harvest: $1.1 E 6 \mathrm{~kg} / \mathrm{yr}$

Dilution Factor: 1

(a) Flow includes fresh water river flow and saline "mixing flow" of estuary as given in FES (1972).

(b) Environment primarlly salt water so invertebrates considered in lleu of drinking water. 
POPULATION DOSE-COMMITMENT ESTIMATES AND

AVERAGE INDIVIDUAL DOSE-COMMITMENT HISTOGRAM FOR

SURRY 1 AND 2

Dose Commitments (person-rem) from Waterborne Pathways

Total Body GI-LII Ihyrotd Bone Liver

$\begin{array}{llllll}\text { Infant } & 0.0 \mathrm{E}+00 & 0.0 \mathrm{E}+00 & 0.0 \mathrm{~F}+00 & 0.0 \mathrm{E}+00 & 0.0 \mathrm{E}+00 \\ \text { Child } & 7.4 \mathrm{E}-02 & 9.7 \mathrm{E}-02 & 4.5 \mathrm{E}-02 & 2.5 \mathrm{E}-01 & 1.6 \mathrm{E}-01 \\ \text { Teen } & 5.1 \mathrm{E}-02 & 1.9 \mathrm{E}-01 & 3.1 \mathrm{E}-02 & 1.4 \mathrm{E}-01 & 1.3 \mathrm{E}-01 \\ \text { Adu1t } & 3.5 \mathrm{E}-01 & 1.7 \mathrm{E}+00 & 2.1 \mathrm{E}-01 & 8.1 \mathrm{E}-01 & 7.3 \mathrm{E}-01 \\ \text { TOTAL } & 4.7 \mathrm{E}-01 & 1.9 \mathrm{E}+00 & 2.8 \mathrm{E}-01 & 1.2 \mathrm{E}+00 & 1.0 \mathrm{E}+00\end{array}$

Dose Commitments (person-rem) from Airborne Pathways

Total Body GI-LII Thyroid Bone Liver Lung

$\begin{array}{lllllll}\text { Infant } & 5.2 \mathrm{E}-04 & 4.9 \mathrm{E}-04 & 7.3 \mathrm{E}-03 & 5.0 \mathrm{E}-04 & 7.9 \mathrm{E}-04 & 6.0 \mathrm{E}-04 \\ \text { Child } & 6.7 \mathrm{E}-03 & 6.4 \mathrm{E}-03 & 4.8 \mathrm{E}-02 & 5.0 \mathrm{E}-03 & 8.5 \mathrm{E}-03 & 7.9 \mathrm{E}-03 \\ \text { Teen } & 4.8 \mathrm{E}-03 & 4.6 \mathrm{E}-03 & 2.2 \mathrm{E}-02 & 2.9 \mathrm{E}-03 & 5.4 \mathrm{E}-03 & 5.9 \mathrm{E}-03 \\ \text { Adult } & 2.8 \mathrm{E}-02 & 2.7 \mathrm{E}-02 & 9.1 \mathrm{E}-02 & 1.5 \mathrm{E}-02 & 2.9 \mathrm{E}-02 & 3.2 \mathrm{E}-02 \\ \text { TOTAL } & 4.0 \mathrm{E}-02 & 3.8 \mathrm{E}-02 & 1.7 \mathrm{E}-01 & 2.4 \mathrm{E}-02 & 4.4 \mathrm{E}-02 & 4.6 \mathrm{E}-02\end{array}$

Production/Consumption factors:

Produce: $0.076 \quad$ Milk: $0.48 \quad$ Meat: 0.39

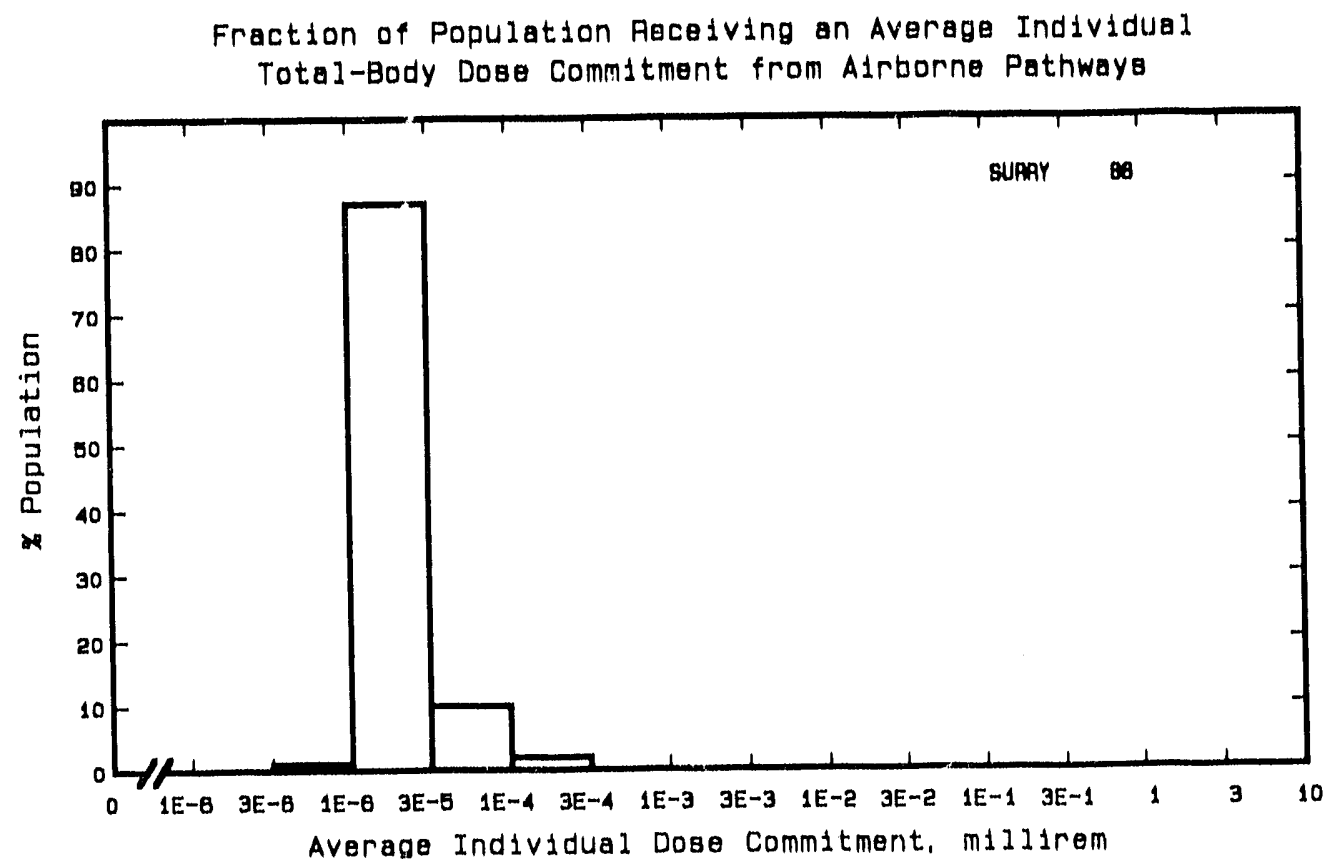


Location: $\quad$ N $41.1000^{\circ} \quad$ W $76.1500^{\circ}$

POPULATION DATA

Total Population Within 2-to-80-km Region: $1.5 \mathrm{E} 6$

Major Metropolitan Centers Within Region:

Center

Wi11 tamsport SMSA

Allentown

Scranton

Wilkes-Barre

Hazleton
Population

118,000

100,000

88,000

52,000

27,000

\section{Location}

$73 \mathrm{~km} \quad W N W$

$79 \mathrm{~km} \quad \mathrm{SE}$

$53 \mathrm{~km} \quad \mathrm{NE}$

$28 \mathrm{~km} \quad \mathrm{NE}$

$21 \mathrm{~km} \quad \mathrm{SE}$

\section{SITE-SPECIFIC DATA - AIRBORNE PATHWAYS}

Average Annual State Production

Of Crops and Animal Products

In $80-\mathrm{km}$ Radius Circle

Regional Productivity Factor:

Animal Grazing Factor:

Meteorology Period of Record: 1 Jan 75 - 31 Dec 75 Recovery: 98\%
Veg: $\quad 5.3 E 7$ kilogram

Milk: 5.3E8 liter

Meat: $5.4 E 7$ kilogram

0.9

0.6

SITE-SPECIFIC DATA - WATERBORNE PATHWAYS VIa SUSQUEHANNA RIVER

Average River Flow at Site: $13,000 \mathrm{ft}^{3} / \mathrm{s}$

Drinking Water:

Exposed Population: 100,000

Dilution Factor: 1

Fish:

Edible Harvest: (a) Dilution Factor: 0.1 (b)

(a) No fish catch data given in FES (1973), so generic consumption rates used (Table A.1).

(b) Ten percent of population consumes fish from river. 


\section{POPULATION DOSE-COMMITMENT ESTIMATES AND AVERAGE INDIVIDUAL DOSE-COMMITMENT HISTOGRAM FOR \\ SUSQUEHANNA 1 AND 2}

Dose Commitments (person-rem) from Waterborne Pathways

Total Body GI-LII Thyroid Bone Liver

$\begin{array}{llllll}\text { Infant } & 1.0 \mathrm{E}-04 & 1.0 \mathrm{E}-04 & 9.3 \mathrm{E}-05 & 1.2 \mathrm{E}-05 & 1.2 \mathrm{E}-04 \\ \text { Child } & 1.9 \mathrm{E}-03 & 1.7 \mathrm{E}-03 & 1.1 \mathrm{E}-03 & 2.3 \mathrm{E}-03 & 4.5 \mathrm{E}-03 \\ \text { Teen } & 1.6 \mathrm{E}-03 & 1.6 \mathrm{E}-03 & 4.1 \mathrm{E}-04 & 1.4 \mathrm{E}-03 & 3.3 \mathrm{E}-03 \\ \text { Adult } & 1.5 \mathrm{E}-02 & 1.5 \mathrm{E}-02 & 3.5 \mathrm{E}-03 & 8.02 \mathrm{E}-03 & 2.1 \mathrm{E}-02 \\ \text { TOTAL } & 1.8 \mathrm{E}-02 & 1.8 \mathrm{E}-02 & 5.1 \mathrm{E}-03 & 1.2 \mathrm{E}-02 & 2.9 \mathrm{E}-02\end{array}$

Dose Commitments (person-rem) from Airborne Pathways

Total Body GI-LII Thyrotd Bone Liver Lung

$\begin{array}{llllllr}\text { Infant } & 2.3 \mathrm{E}-04 & 2.2 \mathrm{E}-04 & 1.2 \mathrm{E}-03 & 4.4 \mathrm{E}-05 & 2.3 \mathrm{E}-04 & 2.3 \mathrm{E}-04 \\ \text { Child } & 3.0 \mathrm{E}-03 & 3.0 \mathrm{E}-03 & 8.7 \mathrm{E}-03 & 4.7 \mathrm{E}-04 & 3.0 \mathrm{E}-03 & 3.1 \mathrm{E}-03 \\ \text { Teen } & 2.0 \mathrm{E}-03 & 2.0 \mathrm{E}-03 & 4.2 \mathrm{E}-03 & 3.4 \mathrm{E}-04 & 2.0 \mathrm{E}-03 & 2.1 \mathrm{E}-03 \\ \text { Adult } & 1.1 \mathrm{E}-02 & 1.1 \mathrm{E}-02 & 1.8 \mathrm{E}-02 & 2.0 \mathrm{E}-03 & 1.1 \mathrm{E}-02 & 1.1 \mathrm{E}-02 \\ \text { TOTAL } & 1.6 \mathrm{E}-02 & 1.6 \mathrm{E}-02 & 3.2 \mathrm{E}-02 & 2.9 \mathrm{E}-03 & 1.6 \mathrm{E}-02 & 1.7 \mathrm{E}-02\end{array}$

Production/Consumption factors:

Produce: 0.16

Milk: 2.4

Meat: 0.41

Fraction of Popllation Receiving an Average Individual Total-Body Dose Commitment from Airborne Pathwaye

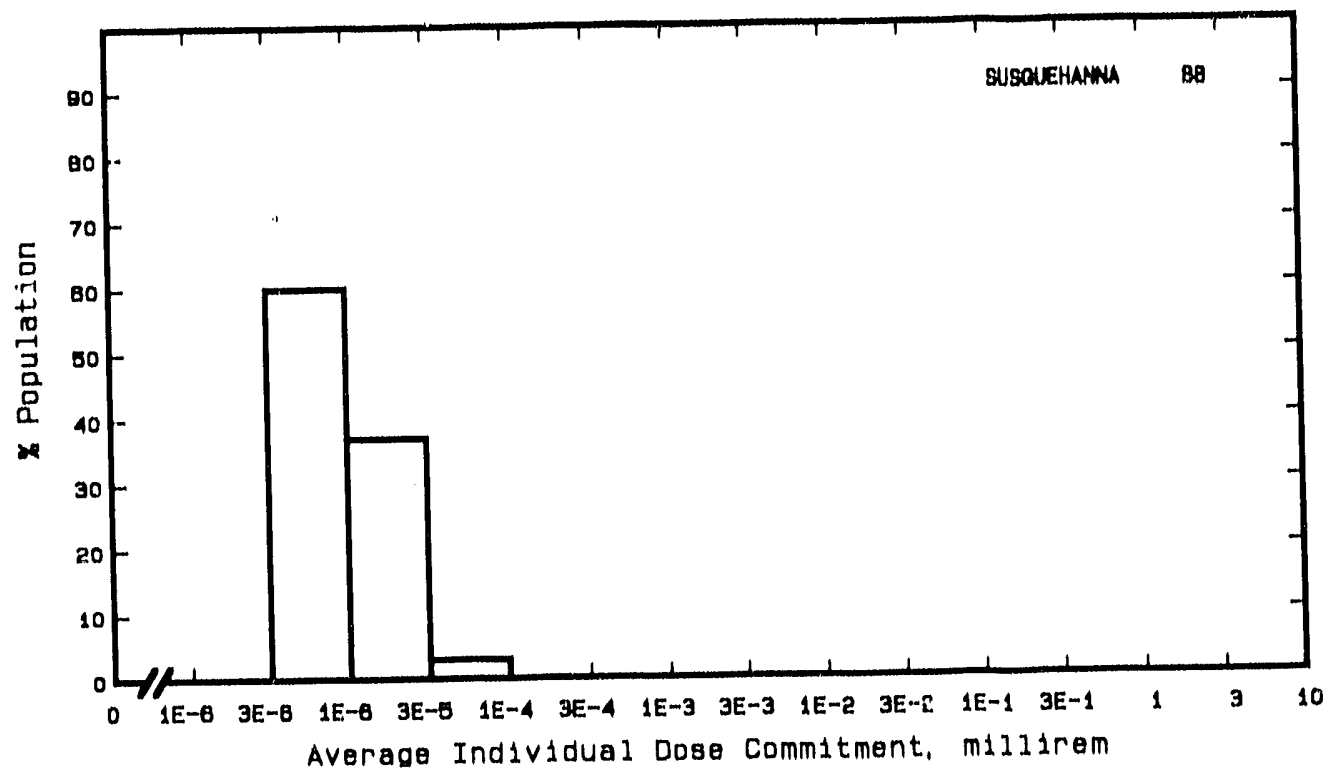


Site: THREE MILE ISLAND

THREE MILE ISLAND, PENNSYLVANIA

Location: $\quad$ N $40.1531^{\circ} \quad$ W $76.7250^{\circ}$

POPULATION DATA

Total Population Within 2-to-80-km Region: 2.1E6

Major Metropolitan Centers Within Region:

$\quad$ Center
Harrisburg SMSA
York SMSA
Lancaster SMSA
Reading SMSA
Lebanon

Population

Location

460,000
390,000
390,000
320,000
26,000

$18 \mathrm{~km} \quad \mathrm{NW}$

$21 \mathrm{~km} \quad \mathrm{~S}$

$38 \mathrm{~km} \quad \mathrm{ESE}$

$71 \mathrm{~km} \quad \mathrm{ENE}$

$33 \mathrm{~km} \quad \mathrm{NE}$

SITE-SPECIFIC DATA - AIRBORNE PATHWAYS

Average Annual State Production

Veg: $5.3 E 7$ kilogram

of Crops and Animal Products

In $80-\mathrm{km}$ Radius Circle

Milk: 5.3E8 liter

Meat: $5.4 E 7$ kilogram

Regional Productivity Factor:

Animal Grazing Factor:

1

0.5

Meteorology Period of Record: 1 OCT 72 - 30 SEP 73 Recovery: $80 \%$

SITE-SPECIFIC DATA - WATERBORNE PATHWAYS VIa SUSQUEHANNA RIVER

Average River Flow

at Site: $34,000 \mathrm{ft}^{3} / \mathrm{s}$

Drinking Water:

Exposed Population: 230,000

Dilution Factor: 1

Fish:

Edible Harvest: (a)

Dilution Factor: 0.025 (b)

(a) No fish catch data given in FES (1972), so generto consumption rates used (Table A-1).

(b) Ten percent of population obtaill $25 \%$ of their fish from river according to FES. 


\section{POPULATION DOSE-COMMITMENT ESTIMATES AND \\ AVERAGE INDIVIDUAL DOSE-COMMITMENT HISTOGRAM FOR \\ THREE MILE ISLAND 1 AND 2}

Dose Coinmitments (person-rem) from Waterborne Pathways (a)

Total Body GI-LII $\underline{\text { Thyroid }}$ Bone Liver

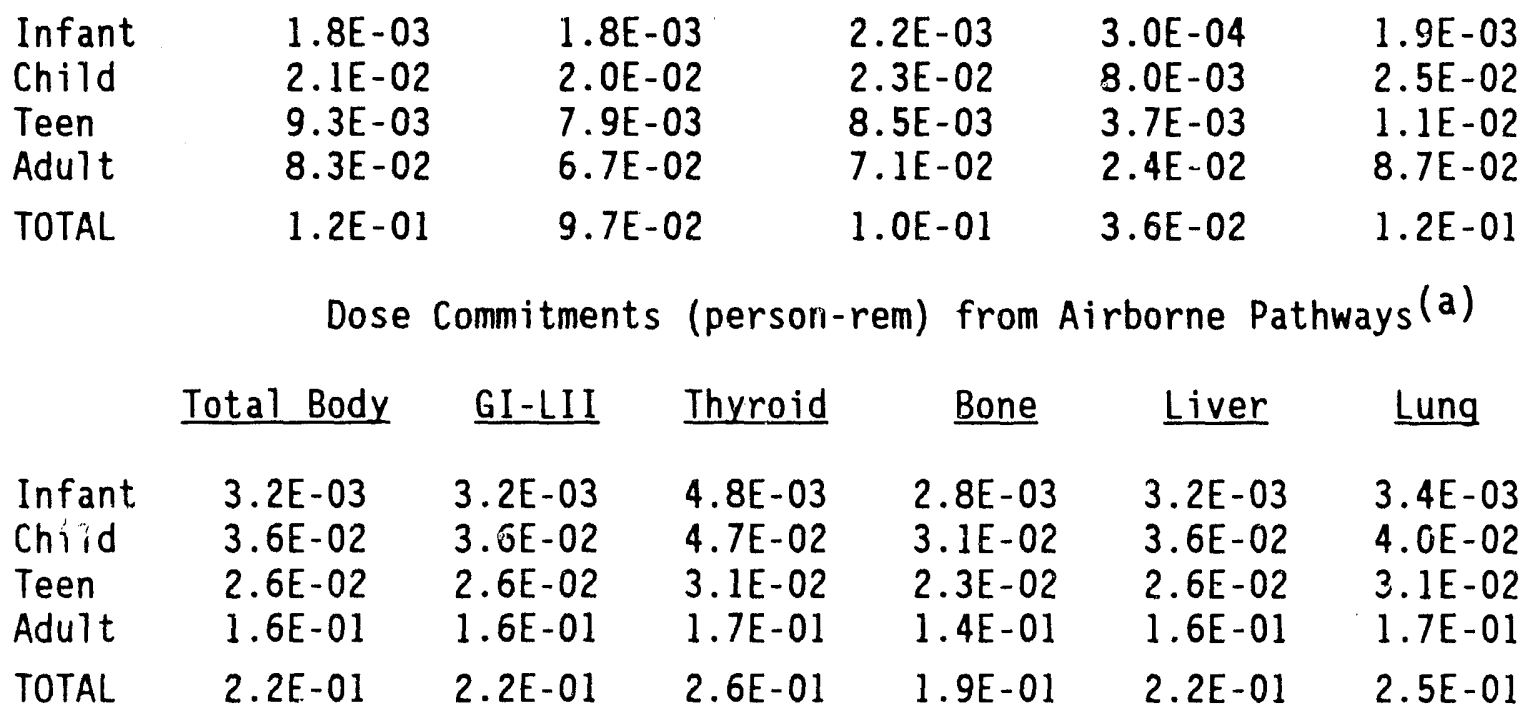

Production/Consumption factors:

Produce: $0.13 \quad$ Milk: 1.9 Meat: 0.32

Fraction of Population Receiving an Average Individual

Total-Body Dose Commitment from Airborne Pathways

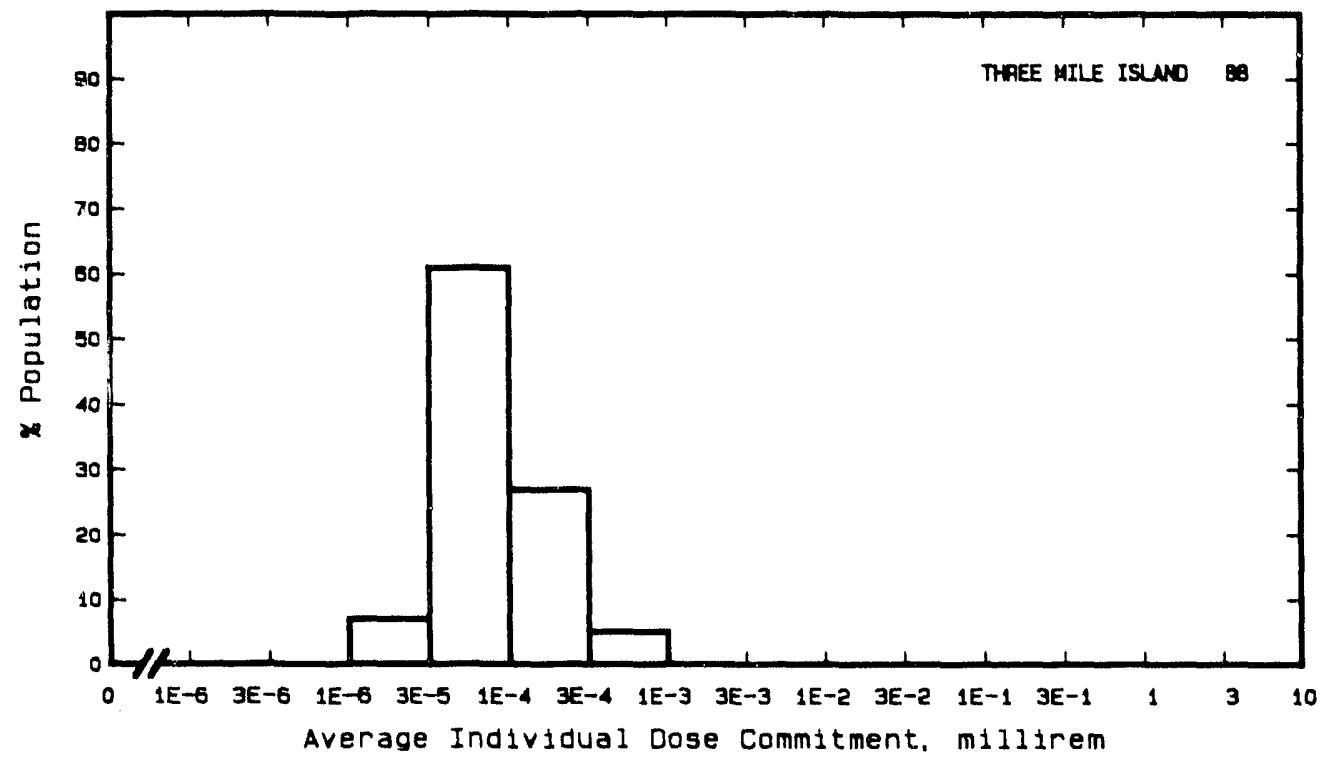

(a) Doses include releases from the TMI 2 / Epicore. 
Site: TROJAN

PRESCOTT, OREGON

Location: $\quad$ N $46.0408^{\circ} \quad$ W $122.8844^{\circ}$

POPULATION DATA

Total Population Within 2-to-80-km Region: 1.5E6

Major Metropolitan Centers Within Region:

Center Population Location

Portland-Vancouver SMSA $\quad 1,500,000 \quad 60 \mathrm{~km} \quad$ SSE

Longview

Astoria

35,000

15,000

Forest Grove

14,000

Centralia

13,000

$12 \mathrm{~km} \quad \mathrm{NNW}$

$72 \mathrm{~km}$ WNW

$58 \mathrm{~km} \quad \mathrm{SSW}$

$75 \mathrm{~km} \mathrm{~N}$

SITE-SPECIFIC DATA - AIRBORNE PATHWAYS

Average Annual State Production

Veg: $6.4 E 7$ kilogram

of Crops and Animal Products

In $80-\mathrm{km}$ Radius Circle

Milk: $3.7 E 7$ liter

Meat: $2.6 \mathrm{E} 7 \mathrm{kilogram}$

Regional Productivity Factor:

0.9

Animal Grazing Factor:

0.75

Meteorology Period of Record: 1 SEP 71 - 31 AUG 74 Recovery: 90\%

SITE-SPECIFIC DATA - WATERBORNE PATHWAYS via COLUMBIA RIVER

Average River Flow

at Site: $230,000 \mathrm{ft}^{3} / \mathrm{s}$

Drinking Water:

Exposed Population: 560 (a)

Dilution Factor: 1

Fish:

Edible Harvest: $1.0 \mathrm{E} 6 \mathrm{~kg} / \mathrm{yr}$

Dilution Factor: 1

(a) Population of Rainier divided by 4 , since residents only there for $25 \%$ of the year. 


\author{
POPULATION DOSE-COMMITMENT ESTIMATES AND \\ AVERAGE INDIVIDUAL DOSE-COMMITMENT HISTOGRAM FOR \\ TROJAN
}

Dose Commitments (person-rem) from Waterborne Pathways

Total Body GI-LII Thyroid Bone Liver

$\begin{array}{llllll}\text { Infant } & 7.9 \mathrm{E}-07 & 8.0 \mathrm{E}-07 & 7.9 \mathrm{E}-07 & 9.6 \mathrm{E}-08 & 7.8 \mathrm{E}-07 \\ \text { Child } & 9.5 \mathrm{E}-05 & 8.1 \mathrm{E}-04 & 3.2 \mathrm{E}-05 & 3.0 \mathrm{E}-04 & 2.6 \mathrm{E}-04 \\ \text { Teen } & 1.1 \mathrm{E}-04 & 1.6 \mathrm{E}-03 & 2.4 \mathrm{E}-05 & 1.9 \mathrm{E}-04 & 2.2 \mathrm{E}-04 \\ \text { Adult } & 1.1 \mathrm{E}-03 & 1.4 \mathrm{E}-02 & 1.9 \mathrm{E}-04 & 1.2 \mathrm{E}-03 & 1.3 \mathrm{E}-03 \\ \text { TOTAL } & 1.3 \mathrm{E}-03 & 1.7 \mathrm{E}-02 & 2.5 \mathrm{E}-04 & 1.7 \mathrm{E}-03 & 1.8 \mathrm{E}-03\end{array}$

Dose Commitments (person-rem) from Airborne Pathways

Iotal Body GI-LII Thyroid Bone Liver Lung

$\begin{array}{lllllll}\text { Infant } & 4.5 \mathrm{E}-04 & 4.5 \mathrm{E}-04 & 1.1 \mathrm{E}-03 & 2.1 \mathrm{E}-04 & 4.5 \mathrm{E}-04 & 4.6 \mathrm{E}-04 \\ \text { Child } & 7.5 \mathrm{E}-03 & 7.5 \mathrm{E}-03 & 1.4 \mathrm{E}-02 & 2.4 \mathrm{E}-03 & 7.5 \mathrm{E}-03 & 7.8 \mathrm{E}-03 \\ \text { Teen } & 5.4 \mathrm{E}-03 & 5.4 \mathrm{E}-03 & 8.5 \mathrm{E}-03 & 1.7 \mathrm{E}-03 & 5.4 \mathrm{E}-03 & 5.8 \mathrm{E}-03 \\ \text { Adult } & 3.2 \mathrm{E}-02 & 3.2 \mathrm{E}-02 & 4.5 \mathrm{E}-02 & 1.0 \mathrm{E}-02 & 3.2 \mathrm{E}-02 & 3.3 \mathrm{E}-02 \\ \text { TOTAL } & 4.5 \mathrm{E}-02 & 4.5 \mathrm{E}-02 & 6.8 \mathrm{E}-02 & 1.5 \mathrm{E}-02 & 4.5 \mathrm{E}-02 & 4.7 \mathrm{E}-02\end{array}$

Production/Consumption factors:

Produce: 0.20 Milk: 0.17 Meat: 0.20

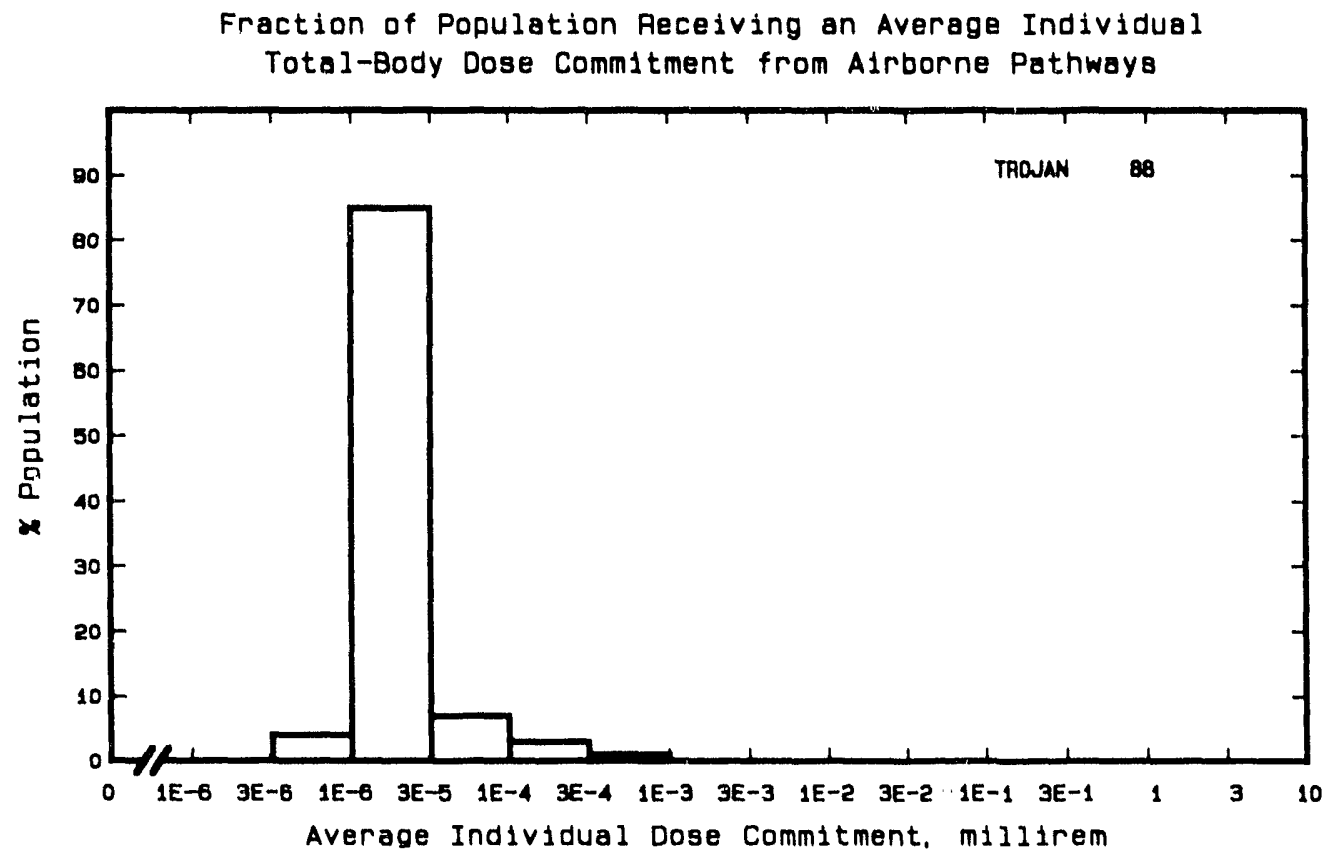


Site: TURKEY POINT

DADE COUNTY, FLORIDA

Location: N $25.4350^{\circ}$ W $80.3314^{\circ}$

POPULATION DATA

Total Population Within 2-to-80-km Region: $2.9 E 6$

Major Metropolitan Centers Within Region:

Center Population Location

Miami-Hialeah SMSA

$2,000,000$

$41 \mathrm{~km} \quad \mathrm{NNE}$

Fort Lauderdale-Hollywood SMSA

Homestead

Upper Keys Division

$1,300,000$

24,000

18,000

$79 \mathrm{~km} \quad \mathrm{NNE}$

$16 \mathrm{~km} \quad \mathrm{~W}$

$42 \mathrm{~km} \mathrm{~S}$

SITE-SPECIFIC DATA - AIRBORNE PATHWAYS

Average Annual State Production

Veg: $2.8 \mathrm{E} 7 \mathrm{kilogram}$

of Crops and inimal Products

In $80-\mathrm{km}$ Radius Circle

Milk: 1.1E8 liter

Meat: $7.2 E 7$ kilogram

Regional Productivity Factor:

Animal Grazing Factor:

0.4

1

Meteorology Period of Record: 1 JAN 73 - 31 DEC 73 Recovery: $98 \%$

SITE-SPECIFIC DATA - WATERBORNE PATHWAYS via BISCAYNE BAY

Average Dilution Flow

from Plants: $660 \mathrm{ft}^{3} / \mathrm{s}$

Fish:

Edible Harvest: (a)

Dilution Factor: 0.001

Invertebrates:

Edible Harvest: (a)

Dilution Factor: 0.002

(a) No catch data given in DES (1972), so generic consumption rates used (Table A-1). 


\section{POPULATION DOSE-COMMITMENT ESTIMATES AND \\ AVERAGE INDIVIDUAL DOSE-COMMITMENT HISTOGRAM FOR \\ TURKEY POINT 3 AND 4}

Dose Commitments (person-rem) from Waterborne Pathways

Total Body GI-LII Thyroid Bone Liver

$\begin{array}{llllll}\text { Infant } & 0.0 \mathrm{E}+00 & 0.0 \mathrm{E}+00 & 0.0 \mathrm{E}+00 & 0.0 \mathrm{E}+00 & 0.0 \mathrm{E}+00 \\ \text { Child } & 7.7 \mathrm{E}-03 & 1.2 \mathrm{E}-02 & 7.7 \mathrm{E}-04 & 1.8 \mathrm{E}-02 & 1.2 \mathrm{E}-02 \\ \text { Teen } & 5.2 \mathrm{E}-03 & 2.4 \mathrm{E}-02 & 5.9 \mathrm{E}-04 & 1.0 \mathrm{E}-02 & 9.6 \mathrm{E}-03 \\ \text { Aduit } & 3.2 \mathrm{E}-02 & 2.1 \mathrm{E}-01 & 4.2 \mathrm{E}-03 & 5.9 \mathrm{E}-02 & 5.6 \mathrm{E}-02 \\ \text { TOTAL } & 4.5 \mathrm{E}-02 & 2.5 \mathrm{E}-01 & 5.5 \mathrm{E}-03 & 8.6 \mathrm{E}-02 & 7.8 \mathrm{E}-02\end{array}$

Dose Commitments (person-rem) from Airborne Pathways

Total Body GI-LII Thyroid Bone Liver Lung

$\begin{array}{lllllll}\text { Infant } & 1.5 \mathrm{E}-03 & 1.5 \mathrm{E}-03 & 5.8 \mathrm{E}-03 & 8.0 \mathrm{E}-04 & 1.5 \mathrm{E}-03 & 1.6 \mathrm{E}-03 \\ \text { Child } & 2.2 \mathrm{E}-02 & 2.2 \mathrm{E}-02 & 4.6 \mathrm{E}-02 & 8.8 \mathrm{E}-03 & 2.2 \mathrm{E}-02 & 2.2 \mathrm{E}-02 \\ \text { Teen } & 1.6 \mathrm{E}-02 & 1.6 \mathrm{E}-02 & 2.6 \mathrm{E}-02 & 6.4 \mathrm{E}-03 & 1.6 \mathrm{E}-02 & 1.7 \mathrm{E}-02 \\ \text { Adult } & 9.7 \mathrm{E}-02 & 9.7 \mathrm{E}-02 & 1.3 \mathrm{E}-01 & 3.9 \mathrm{E}-02 & 9.8 \mathrm{E}-02 & 1.0 \mathrm{E}-01 \\ \text { TOTAL } & 1.4 \mathrm{E}-01 & 1.4 \mathrm{E}-01 & 2.1 \mathrm{E}-01 & 5.5 \mathrm{E}-02 & 1.4 \mathrm{E}-01 & 1.4 \mathrm{E}-01\end{array}$

Production/Consumption factors:

Produce: 0.020 Milk: 0.12 Meat: 0.12

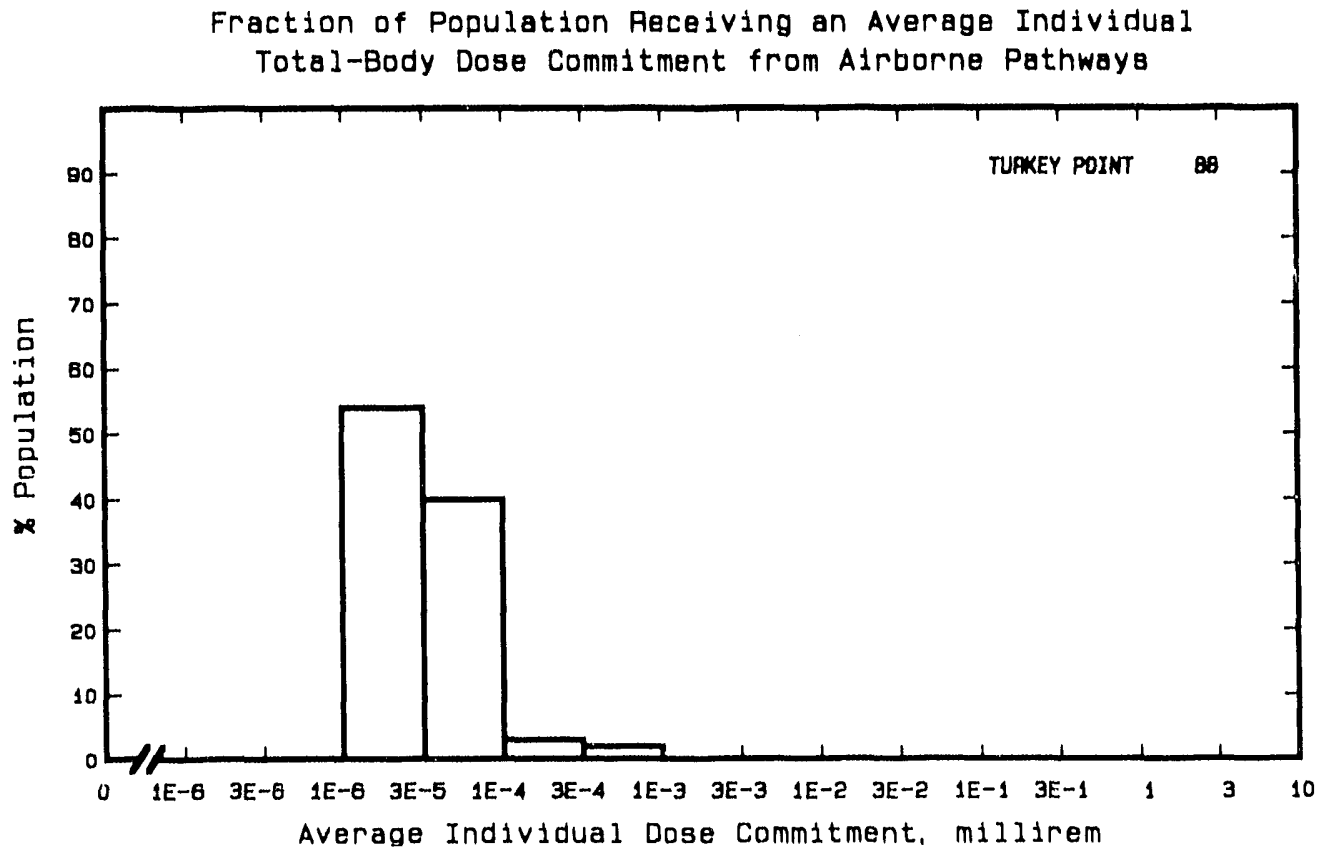


Site: VERMONT YANKEE

VERNON, VERMONT

Location: $\quad$ N $42.7803^{\circ}$ W $72.5158^{\circ}$

POPULATION DATA

Total Population Within 2-to-80-km Region: 1.4E6

Major Metropolitan Centers Within Region:

Center Population Location

Springfield-Chicopee-Holyoke SMSA worcester SMSA (1/2)

Pittsfield

Fitchburg

570,000

200,000

100,000

Leominster

44,000

39,000

Keene

22,000

$\begin{array}{lll}70 & \mathrm{~km} & \mathrm{~S} \\ 80 & \mathrm{~km} & \text { SE } \\ 71 & \mathrm{~km} & \text { SW } \\ 63 & \mathrm{~km} & \text { ESE } \\ 68 \mathrm{~km} & \text { ESE } \\ 26 & \mathrm{~km} & \text { NW }\end{array}$

SITE-SPECIFIC DATA - AIRBORNE PATHWAYS

Average Annual State Production

Veg: 4.4E6 kilogram

of Crops and Animal Products

In $80-\mathrm{km}$ Radius Circle

Milk: 7.3E8 Titer

Meat: 2.7 E7 kilogram

Regional Productivity Factor:

Animal Grazing Factor:

1

0.4

Meteorology Period of Record: 1 APR 75 - 31 MAR 76 Recovery: 97\%

Average River Flow at Site: $10,000 \mathrm{ft}^{3} / \mathrm{s}$

Drinking Water: None

Fish: $\quad$ None 


\section{POPULATION DOSE-COMMITMENT ESTIMATES AND \\ AVERAGE INDIVIDUAL DOSE-COMMITMENT HISTOGRAM FOR \\ VERMONT YANKEE \\ Dose Commitments (person-rem) from Waterborne Pathways \\ Total Body GI-LII Thyroid Bone Liver}

Infant

Child

Teen

Adult

(No Waterborne Pathway Doses) (a)

TOTAL

Dose Commitments (person-rem) from Airborne Pathways

Total Body GI-LII Thyroid Bone Liver Lung

$\begin{array}{lllllll}\text { Infant } & 9.3 \mathrm{E}-04 & 9.2 \mathrm{E}-04 & 3.2 \mathrm{E}-03 & 5.9 \mathrm{E}-05 & 9.5 \mathrm{E}-04 & 1.0 \mathrm{E}-03 \\ \text { Child } & 1.1 \mathrm{E}-02 & 1.1 \mathrm{E}-02 & 2.6 \mathrm{E}-02 & 6.1 \mathrm{E}-04 & 1.1 \mathrm{E}-04 & 1.3 \mathrm{E}-02 \\ \text { Teen } & 7.4 \mathrm{E}-03 & 7.4 \mathrm{E}-03 & 1.5 \mathrm{E}-02 & 3.6 \mathrm{E}-04 & 7.4 \mathrm{E}-03 & 9.2 \mathrm{E}-03 \\ \text { Adult } & 3.9 \mathrm{E}-02 & 4.0 \mathrm{E}-02 & 6.9 \mathrm{E}-02 & 1.9 \mathrm{E}-03 & 4.0 \mathrm{E}-02 & 4.7 \mathrm{E}-02 \\ \text { TOTAL } & 5.9 \mathrm{E}-02 & 5.9 \mathrm{E}-02 & 1.1 \mathrm{E}-01 & 2.9 \mathrm{E}-03 & 5.9 \mathrm{E}-02 & 7.1 \mathrm{E}-02\end{array}$

Production/Crinsumption factors:

Produce: $0.015 \quad$ Milk: $3.8 \quad$ Meat: 0.23

Fraction of Population Receiving an Average Individual

Total-Body Dose Commitment from Alrborne Pathways

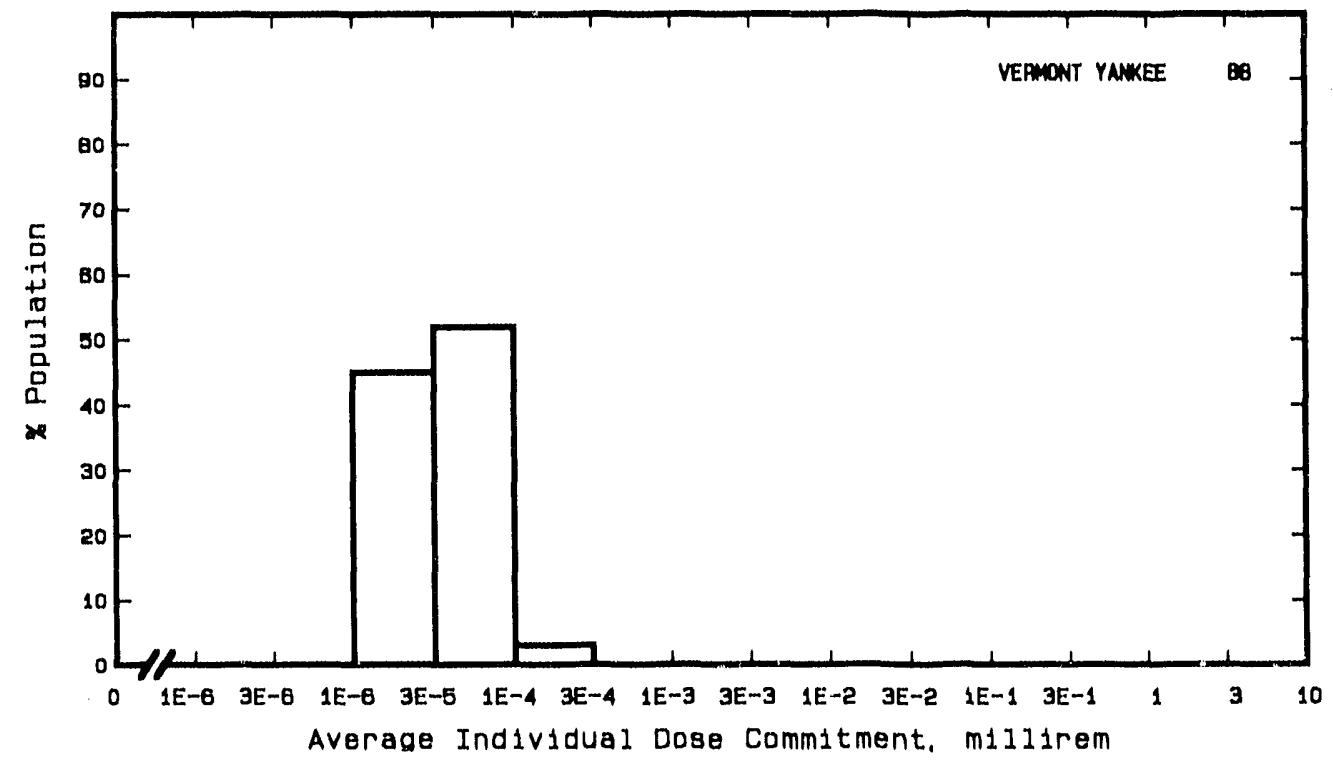

(a) No liquid releases reported (Tichler, et a1, 1991), 
Site: VOGTLE

WAYNESBORO, GEORGIA

Location: $\quad$ N $33.1419^{\circ} \quad$ W $81.7647^{\circ}$

POPULATION DATA

Total Population Within 2-to-80-km Region: 6.2 E5

Major Metropolitan Centers Within Region:

Center

Population

Location

$\begin{array}{lrll}\text { Augusta SMSA } & 400,000 & 40 \mathrm{~km} & \text { NNW } \\ \text { Fort Gordon } & 18,000 & 48 \mathrm{~km} & \mathrm{NW} \\ \text { Aiken } & 17,000 & 45 \mathrm{~km} & \mathrm{~N} \\ \text { Statesboro } & 17,000 & 76 \mathrm{~km} & \mathrm{~S}\end{array}$

SITE-SPECIFIC DATA - AIRBORNE PATHWAYS

Average Annual State Production

of Crops and Animal Products

In $80-\mathrm{km}$ Radius Circle

Regional Productivity Factor:

Animal Grazing Factor:
Veg: $8.8 \mathrm{E} 6$ kilogram

Milk: 7.0E7 liter

Meat: $8.1 \mathrm{E7} \mathrm{kilogram}$

0.8

0.75

Meteorology Period of Record: 4 APR 77 - 31 MAR 81 Recovery: $93 \%$

\section{SITE-SPECIFIC DATA - WATERBORNE PATHWAYS via SAVANNAH RIVER}

Average River Flow at Site: $10,000 \mathrm{ft}^{3} / \mathrm{s}$

Drinking Water: None

Fish:

Edible Harvest: $0.18 \mathrm{~kg} / \mathrm{yr}(\mathrm{a})$

Dilution Factor: 1

(a) Ten percent of population are assumed to obtain $f($ sh from river. Average individual consumption rate of $5 \mathrm{~g} / \mathrm{d}$ given in FES (1974) used in lieu of catch data. 


\section{POPULATION DOSE-COMMITMENT ESTIMATES AND \\ AVERAGE INDIVIDUAL DOSE-COMMITMENT HISTOGRAM FOR \\ VOGTLE 1}

Dose Commitments (person-rem) from Waterborne Pathways

\begin{tabular}{|c|c|c|c|c|c|}
\hline & Total Body & GI-LLI & Thyroid & Bone & Liver \\
\hline $\begin{array}{l}\text { Infant } \\
\text { Child } \\
\text { Teen } \\
\text { Adult }\end{array}$ & $\begin{array}{l}0.0 E+00 \\
4.3 E-04 \\
3.2 E-04 \\
2.1 E-03\end{array}$ & $\begin{array}{l}0.0 E+00 \\
1.1 E-02 \\
2.2 E-02 \\
1.9 E-01\end{array}$ & $\begin{array}{l}0.0 E+00 \\
1.5 E-04 \\
1.2 E-04 \\
8.5 E-04\end{array}$ & $\begin{array}{l}0.0 \mathrm{E}+00 \\
3.3 \mathrm{E}-04 \\
1.9 \mathrm{E}-04 \\
1.2 \mathrm{E}-03\end{array}$ & $\begin{array}{l}0.0 E+00 \\
5.8 E-04 \\
5.1 E-04 \\
3.2 E-03\end{array}$ \\
\hline TOTAL & $2.9 E-03$ & 2. $2 E-01$ & $1.1 \mathrm{E}-03$ & $1.7 \mathrm{E}-03$ & 4. $3 E-03$ \\
\hline
\end{tabular}

Dose Commitments (person-rem) from Airborne Pathways

Total Body GI-LLI Thyrotd Bone Liver Lung

$\begin{array}{lllllll}\text { Infant } & 2.2 \mathrm{E}-04 & 2.2 \mathrm{E}-04 & 2.2 \mathrm{E}-04 & 3.1 \mathrm{E}-05 & 2.2 \mathrm{E}-04 & 2.2 \mathrm{E}-04 \\ \text { Child } & 3.2 \mathrm{E}-03 & 3.2 \mathrm{E}-03 & 3.2 \mathrm{E}-03 & 3.5 \mathrm{E}-04 & 3.2 \mathrm{E}-03 & 3.2 \mathrm{E}-03 \\ \text { Teen } & 2.1 \mathrm{E}-03 & 2.1 \mathrm{E}-03 & 2.1 \mathrm{E}-03 & 2.6 \mathrm{E}-04 & 2.1 \mathrm{E}-03 & 2.2 \mathrm{E}-03 \\ \text { Adult } & 1.2 \mathrm{E}-02 & 1.2 \mathrm{E}-02 & 1.2 \mathrm{E}-02 & 1.5 \mathrm{E}-03 & 1.2 \mathrm{E}-02 & 1.3 \mathrm{E}-02 \\ \text { TOTAL } & 1.8 \mathrm{E}-02 & 1.8 \mathrm{E}-02 & 1.8 \mathrm{E}-02 & 2.2 \mathrm{E}-03 & 1.8 \mathrm{E}-02 & 1.8 \mathrm{E}-02\end{array}$

Production/Cunsumption factors:

Produce: $0.058 \quad$ Milk: $0.69 \quad$ Meat: 1.3

Fraction of Population Receiving an Average Individual Total-Body Dose Commitment from Alrborne Pathwaya

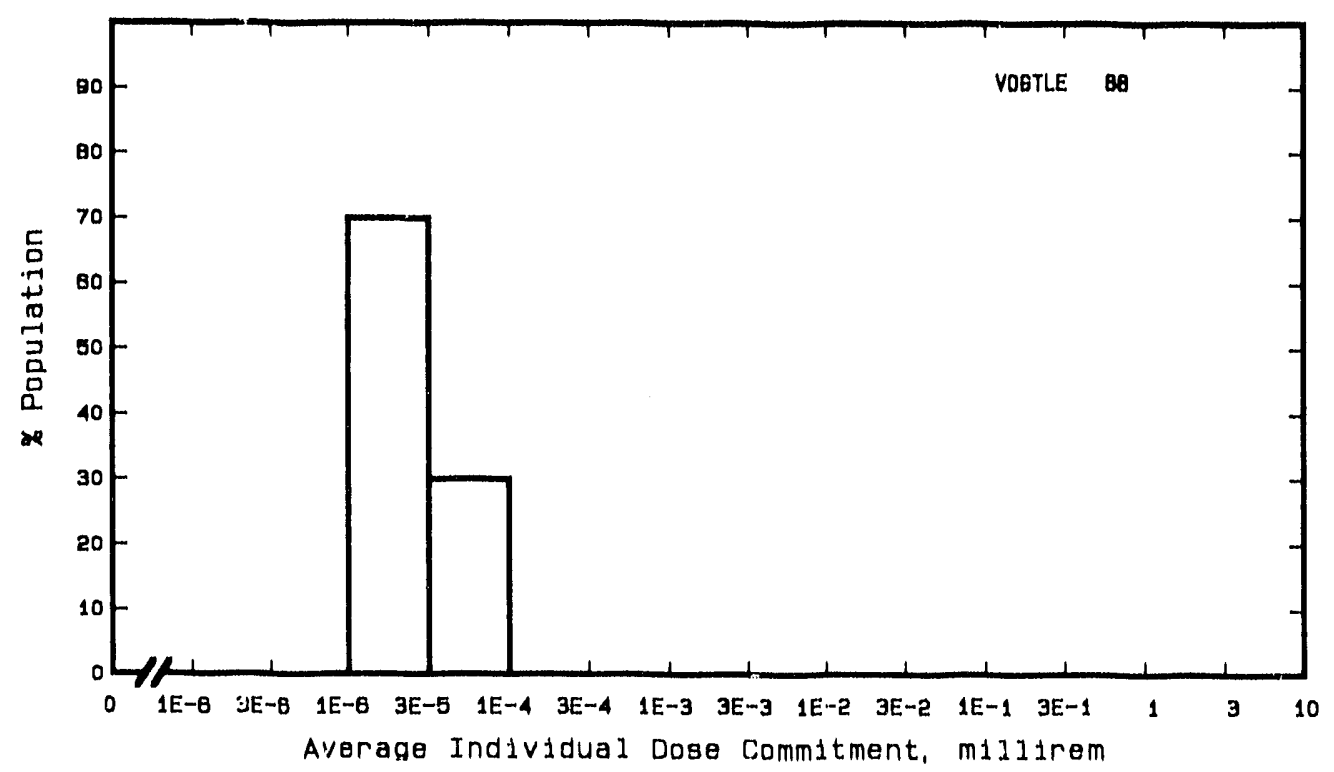


Site: WATERFORD

TAFT, LOUISIANA

Location: $\quad$ N $29.9953^{\circ} \quad$ W $90.4728^{\circ}$

POPULATION DATA

Total Population Within 2-to-80-km Region: 1.8E6

Major Metropolitan Centers Within Region:

Center

New Orleans MSA

Metaire

Kenner

Marrero

Houma
Population

$$
1,300,000
$$

140,000

30,000

29,000

31,000

\section{Location}

$32 \mathrm{~km} \quad \mathrm{E}$

$26 \mathrm{~km} \quad \mathrm{E}$

$16 \mathrm{~km} \quad \mathrm{E}$

$32 \mathrm{~km}$ ESE

$51 \mathrm{~km} \quad \mathrm{SSW}$

SITE-SPECIFIC DATA - AIRBORNE PATHWAYS

Average Annual State Production

of Crops and Animal Products

In $80-\mathrm{km}$ Radius Circle

Regional Productivity Factor:

Animal Grazing Factor:
Veg: $1.8 \mathrm{E} 6 \mathrm{k}+$ logram

Milk: 7.8E7 1iter

Meat: $6.1 E 7$ kflogram

0.6

1.0

Meteorology Period of Record: 2 FEB 77 - 1 FEB 78 Recovery: 89\%

SITE-SPECIFIC DATA - WATERBORNE PATHWAYS via MISSISSIPPI RIVER and GULF

Drinking Water:

Average River Flow

at Site: $490,000 \mathrm{ft}^{3} / \mathrm{s}$

Exposed Population: $1,100,000$

Dilution Factor: 1

Fish:

Edible Harvest: $4.5 E 3 \mathrm{~kg} / \mathrm{yr}$ (River)

Dilution Factor: 1

Edible Harvest: $9.1 \mathrm{E5} \mathrm{kg} / \mathrm{yr}$ (Gulf)

Di7ution Factor: 0.1

Gulf Invertebrates:

Edible Harvest: $5.1 \mathrm{E} 6 \mathrm{~kg} / \mathrm{yr}$ Dilution Factor: 0.1 
POPULATION DOSE-COMMITMENT ESTIMATES AND

AVERAGE INDIVIDUAL DOSE-COMMITMENT HISTOGRAM FOR

\section{WATERFORD 3}

Dose Commitments (person-rem) from Waterborne Pathways

\section{Iotal Body}

Infant

Child

Teen

Adult

TOTAL

$\begin{array}{lllll}1.0 \mathrm{E}-03 & 9.9 \mathrm{E}-04 & 8.4 \mathrm{E}-03 & 5.2 \mathrm{E}-04 & 1.6 \mathrm{E}-03 \\ 1.2 \mathrm{E}-02 & 1.2 \mathrm{E}-02 & 6.2 \mathrm{E}-02 & 6.2 \mathrm{E}-03 & 1.6 \mathrm{E}-02 \\ 5.1 \mathrm{E}-03 & 6.2 \mathrm{E}-03 & 2.0 \mathrm{E}-02 & 1.8 \mathrm{E}-03 & 6.5 \mathrm{E}-03 \\ 4.5 \mathrm{E}-03 & 5.4 \mathrm{E}-02 & 1.5 \mathrm{E}-01 & 1.2 \mathrm{E}-02 & 5.0 \mathrm{E}-02 \\ 6.3 \mathrm{E}-02 & 7.3 \mathrm{E}-02 & 2.4 \mathrm{E}-01 & 2.0 \mathrm{E}-02 & 7.6 \mathrm{E}-02\end{array}$

Dose Commitments (person-rem) from Atrborne Pathways

$$
\text { Total Body GI-LLI Ihyrotd Bone Liver Lung }
$$

$\begin{array}{lllllll}\text { Infant } & 6.0 \mathrm{E}-03 & 6.0 \mathrm{E}-03 & 6.4 \mathrm{E}-03 & 4.3 \mathrm{E}-03 & 6.0 \mathrm{E}-03 & 6.4 \mathrm{E}-03 \\ \text { Child } & 7.6 \mathrm{E}-02 & 7.6 \mathrm{E}-02 & 7.8 \mathrm{E}-02 & 4.8 \mathrm{E}-02 & 7.6 \mathrm{E}-02 & 8.1 \mathrm{E}-02 \\ \text { Teen } & 5.6 \mathrm{E}-02 & 5.6 \mathrm{E}-02 & 5.8 \mathrm{E}-02 & 3.5 \mathrm{E}-02 & 5.6 \mathrm{E}-02 & 6.4 \mathrm{E}-02 \\ \text { Adult } & 3.4 \mathrm{E}-01 & 3.4 \mathrm{E}-01 & 3.4 \mathrm{E}-01 & 2.1 \mathrm{E}-01 & 3.4 \mathrm{E}-01 & 3.6 \mathrm{E}-01 \\ \text { TOTAL } & 4.8 \mathrm{E}-01 & 4.8 \mathrm{E}-01 & 4.9 \mathrm{E}-01 & 3.0 \mathrm{E}-01 & 4.8 \mathrm{E}-01 & 5.1 \mathrm{E}-01\end{array}$

Production/Consumption factors:

Produce: 0.0030 Milk: 0.20 Meat: 0.25

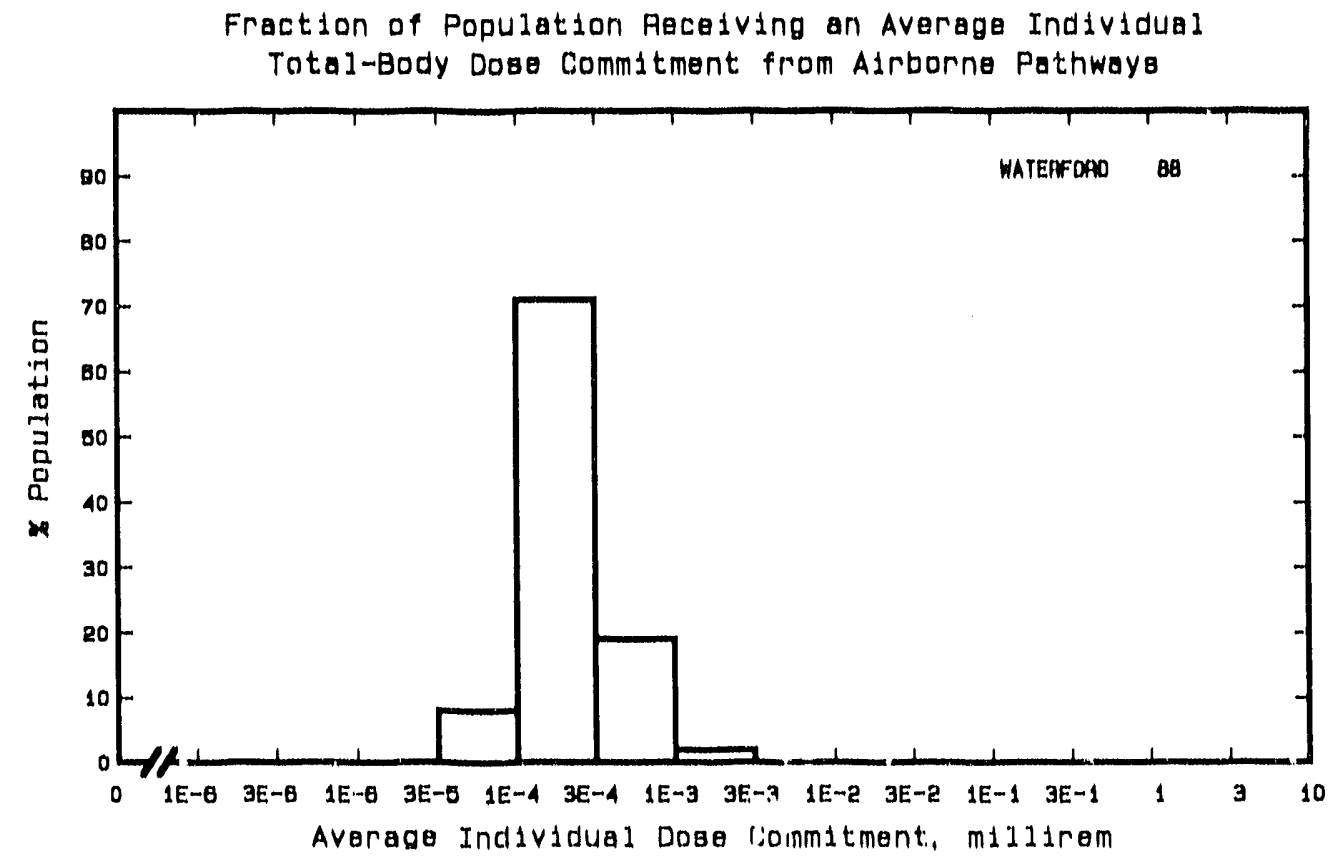


Site: WNP-2
Location:
N $46.2833^{\circ}$
W $119.2916^{\circ}$

RICHLAND, WASHINGTON

POPULATION DATA

Total Population Within 2-to-80-km Region: $2.8 E 5$

Major Metropolitan Centers Within Region:

Center Population Location

$\begin{array}{lllll}\text { Richland } & 36,000 & 14 \mathrm{~km} & \text { S } \\ \text { Kennewick } & 37,000 & 26 \mathrm{~km} & \text { SSE } \\ \text { Pasco } & 19,000 & 28 \mathrm{~km} & \text { SSE } \\ \text { Moses Lake } & 11,000 & 73 \mathrm{~km} & \mathrm{~N}\end{array}$

SITE-SPECIFIC DATA - AIRBORNE PATHWAYS

Average Annual State Production

of Crops and Animal Products

In $80-\mathrm{km}$ Radius Circle

Regional Productivity Factor:

Antmal Grazing Factor:

Meteorology Period of Record: 1 APR 75 - 31 MAR 76 Recovery: $93 \%$
Veg: $2.1 E 8$ kflogram

Milk: 1.2E8 liter

Meat: $3.2 \mathrm{E} 7 \mathrm{kilogram}$

0.8

0.7

SITE-SPECIFIC DATA - WATERBORNE PATHWAYS via COLUMBIA RIVER

Average River Flow

at Site: $115,000 \mathrm{ft}^{3} / \mathrm{s}$

Drinking Water:

Exposed Population: 75,000

Cllution Factor: 3.4 (a)

Fish:

Edible Harvest: $7.5 \mathrm{E} 3 \mathrm{~kg} / \mathrm{yr}$

Dilution Factor: 1

(a) Effective factor for the cities of Richland, Kennewick, and Pasco accounting for only partial dflution. 
POPULATION DOSE-COMMITMENT ESTIMATES AND

AVERAGE INDIVIDUAL DOSE-COMMIIMENT HISTOGRAM FOR

WNP-2

Dose Commitments (person-rem) from Waterborne Pathways

Iotal Body GI-LII Ihyroid Bone Liver

$\begin{array}{llllll}\text { Infant } & 3.4 \mathrm{E}-06 & 3.6 \mathrm{E}-06 & 1.9 \mathrm{E}-05 & 3.0 \mathrm{E}-06 & 6.1 \mathrm{E}-06 \\ \text { Child } & 4.2 \mathrm{E}-05 & 3.8 \mathrm{E}-05 & 1.4 \mathrm{E}-05 & 3.4 \mathrm{E}-05 & 6.4 \mathrm{E}-05 \\ \text { Teen } & 1.8 \mathrm{E}-05 & 1.9 \mathrm{E}-05 & 4.6 \mathrm{E}-05 & 9.7 \mathrm{E}-06 & 2.5 \mathrm{E}-05 \\ \text { Adu1t } & 1.6 \mathrm{E}-04 & 1.7 \mathrm{E}-04 & 3.4 \mathrm{E}-04 & 6.3 \mathrm{E}-05 & 1.9 \mathrm{E}-04 \\ \text { TOTAL } & 2.2 \mathrm{E}-04 & 2.3 \mathrm{E}-04 & 5.5 \mathrm{E}-04 & 1.1 \mathrm{E}-04 & 2.8 \mathrm{E}-04\end{array}$

Dose Commitments (person-rem) from Atrborne Pathways

Total Body GI-LII Ihyrotd Bone Liver Lung

$\begin{array}{lllllll}\text { Infant } & 4.8 \mathrm{E}-04 & 5.7 \mathrm{E}-04 & 1.7 \mathrm{E}-02 & 4.4 \mathrm{E}-04 & 6.8 \mathrm{E}-04 & 4.0 \mathrm{E}-04 \\ \text { Child } & 5.8 \mathrm{E}-03 & 4.4 \mathrm{E}-03 & 1.5 \mathrm{E}-01 & 5.1 \mathrm{E}-03 & 7.2 \mathrm{E}-03 & 5.0 \mathrm{E}-03 \\ \text { Teen } & 3.5 \mathrm{E}-03 & 3.5 \mathrm{E}-03 & 6.3 \mathrm{E}-02 & 3.1 \mathrm{E}-03 & 4.4 \mathrm{E}-03 & 3.9 \mathrm{E}-03 \\ \text { Aduit } & 1.9 \mathrm{E}-02 & 2.0 \mathrm{E}-02 & 2.4 \mathrm{E}-01 & 1.7 \mathrm{E}-02 & 2.2 \mathrm{E}-02 & 2.1 \mathrm{E}-02 \\ \text { TOTAL } & 2.9 \mathrm{E}-02 & 2.9 \mathrm{E}-02 & 4.7 \mathrm{E}-01 & 2.6 \mathrm{E}-02 & 3.4 \mathrm{E}-02 & 3.0 \mathrm{E}-02\end{array}$

Production/Consumption factors:
Produce:
3.1
Mi1k:
2.7
Meat: 1.1

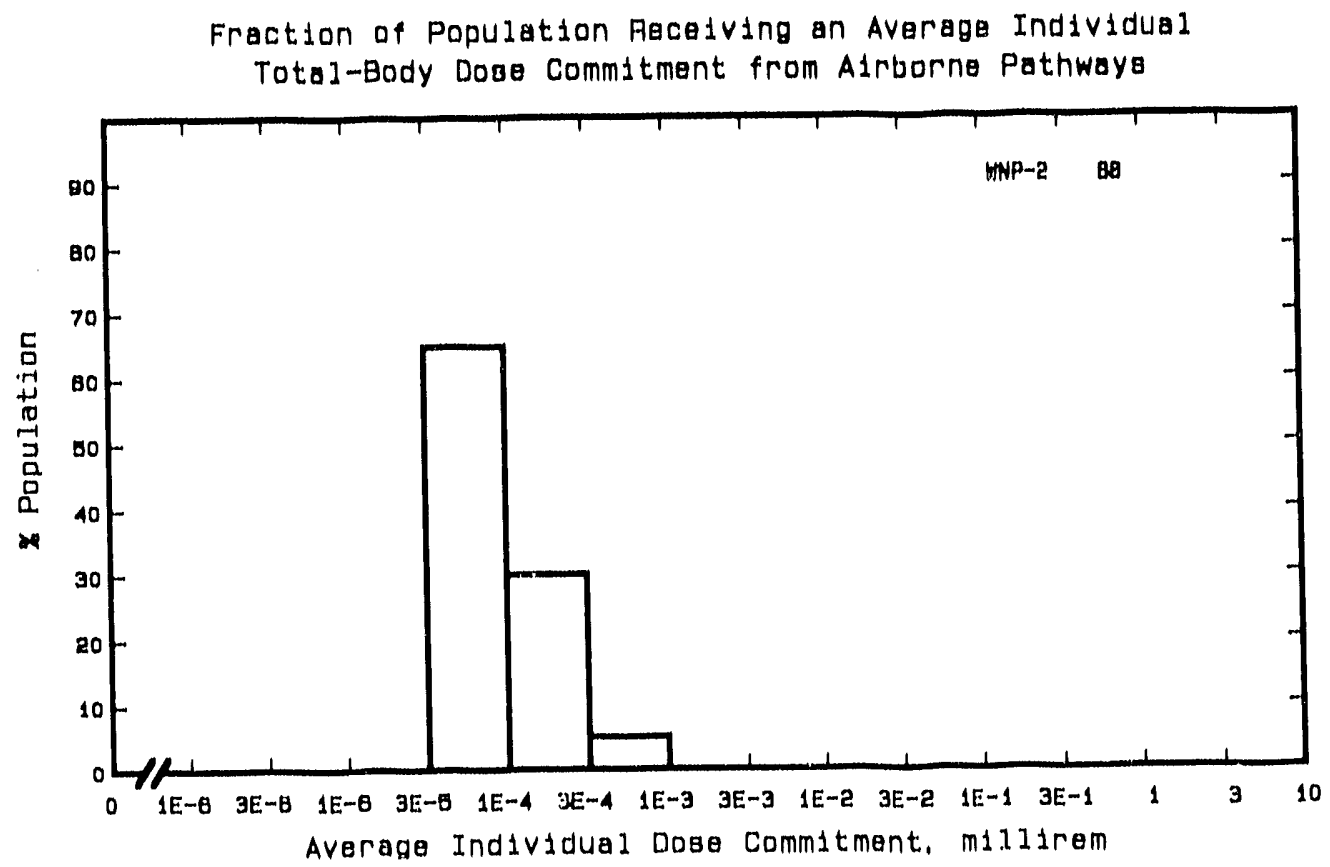


Stte: WOLF CREEK

BURLINGTON, KANSAS

Location: $\quad N 39.0267^{\circ} \quad W 84.7233^{\circ}$

POPULATION DATA

Total Population Within 2-to-80-km Region: 1.9E5

Major Metropolitan Centers Within Region:

Center

Chanute

Emporia

ottawa
Population

11,000

26,000

11,000
Location

$62 \mathrm{~km} \quad \mathrm{SSE}$

$42 \mathrm{~km} \quad W N W$

$58 \mathrm{~km} \quad \mathrm{NW}$

SITE-SPECIFIC DATA - AIRBORNE PATHWAYS

Average Annual State Production

of Crops and Animal Products

In $80-\mathrm{km}$ Radius circle

Regional Productivity Factor:

Animal Grazing Factor:

Meteorology Period of Record: 3 years
Veg: $2.4 \mathrm{E} 8 \mathrm{kilogram}$

Milk: $6.5 E 7$ 1 iter

Meat: $1.6 \mathrm{E} 8 \mathrm{ktlogram}$

0.95

0.5

Recovery: $93 \%$

SITE-SPECIFIC DATA - WATERBORNE PATHWAYS via NEOSHO RIVER

\section{Average River Flow} at Site: $1,300 \mathrm{ft}^{3} / \mathrm{s}$

Drinking Water:

None

Fish:

Edible Harvest: $1.8^{(a)} \mathrm{kg} / \mathrm{yr}$ Dilution Factor: 1

(a) Average individual consumption rate of $5 \mathrm{~g} / \mathrm{d}$ given in FES (1982). 


\section{POPULATION DOSE-COMMITMENT ESTIMATES AND \\ AVERAGE INDIVIDUAL DOSE-COMMITMENT HISTOGRAM FOR \\ WOLF CREEK}

Dose Commitments (person-rem) from Waterborne Pathways

Total Body GI-LLI Thyroid Bone Liver

$\begin{array}{llllll}\text { Infant } & 0.0 \mathrm{E}+00 & 0.0 \mathrm{E}+00 & 0.0 \mathrm{E}+00 & 0.0 \mathrm{E}+00 & 0.0 \mathrm{E}+00 \\ \text { Child } & 1.0 \mathrm{E}-02 & 4.4 \mathrm{E}-02 & 2.4 \mathrm{E}-03 & 3.5 \mathrm{E}-02 & 4.1 \mathrm{E}-02 \\ \text { Teen } & 1.5 \mathrm{E}-02 & 9.1 \mathrm{E}-02 & 2.02 \mathrm{E}-03 & 2.1 \mathrm{E}-02 & 3.5 \mathrm{E}-02 \\ \text { Adult } & 1.5 \mathrm{E}-01 & 7.9 \mathrm{E}-01 & 1.4 \mathrm{E}-02 & 1.2 \mathrm{E}-01 & 2.1 \mathrm{E}-01 \\ \text { TOTAL } & 1.8 \mathrm{E}-01 & 9.2 \mathrm{E}-01 & 1.9 \mathrm{E}-02 & 1.8 \mathrm{E}-01 & 2.9 \mathrm{E}-01\end{array}$

Dose Commitments (person-rem) from Airborne Pathways

Total Body GI-LLI Thyroid Bone Liver Lung

$\begin{array}{lllllll}\text { Infant } & 1.1 \mathrm{E}-04 & 1.1 \mathrm{E}-04 & 1.1 \mathrm{E}-04 & 2.8 \mathrm{E}-05 & 1.1 \mathrm{E}-04 & 1.1 \mathrm{E}-04 \\ \text { Child } & 2.2 \mathrm{E}-03 & 2.2 \mathrm{E}-03 & 2.2 \mathrm{E}-03 & 3.2 \mathrm{E}-04 & 2.2 \mathrm{E}-03 & 2.2 \mathrm{E}-03 \\ \text { Teen } & 1.3 \mathrm{E}-03 & 1.3 \mathrm{E}-03 & 1.3 \mathrm{E}-03 & 2.3 \mathrm{E}-04 & 1.3 \mathrm{E}-03 & 1.3 \mathrm{E}-03 \\ \text { Adult } & 7.0 \mathrm{E}-03 & 7.0 \mathrm{E}-03 & 7.0 \mathrm{E}-03 & 1.4 \mathrm{E}-03 & 7.0 \mathrm{E}-03 & 7.1 \mathrm{E}-03 \\ \text { TOTAL } & 1.1 \mathrm{E}-02 & 1.1 \mathrm{E}-02 & 1.1 \mathrm{E}-02 & 2.02 \mathrm{E}-03 & 1.1 \mathrm{E}-02 & 1.1 \mathrm{E}-02\end{array}$

Production/Consumption factors:

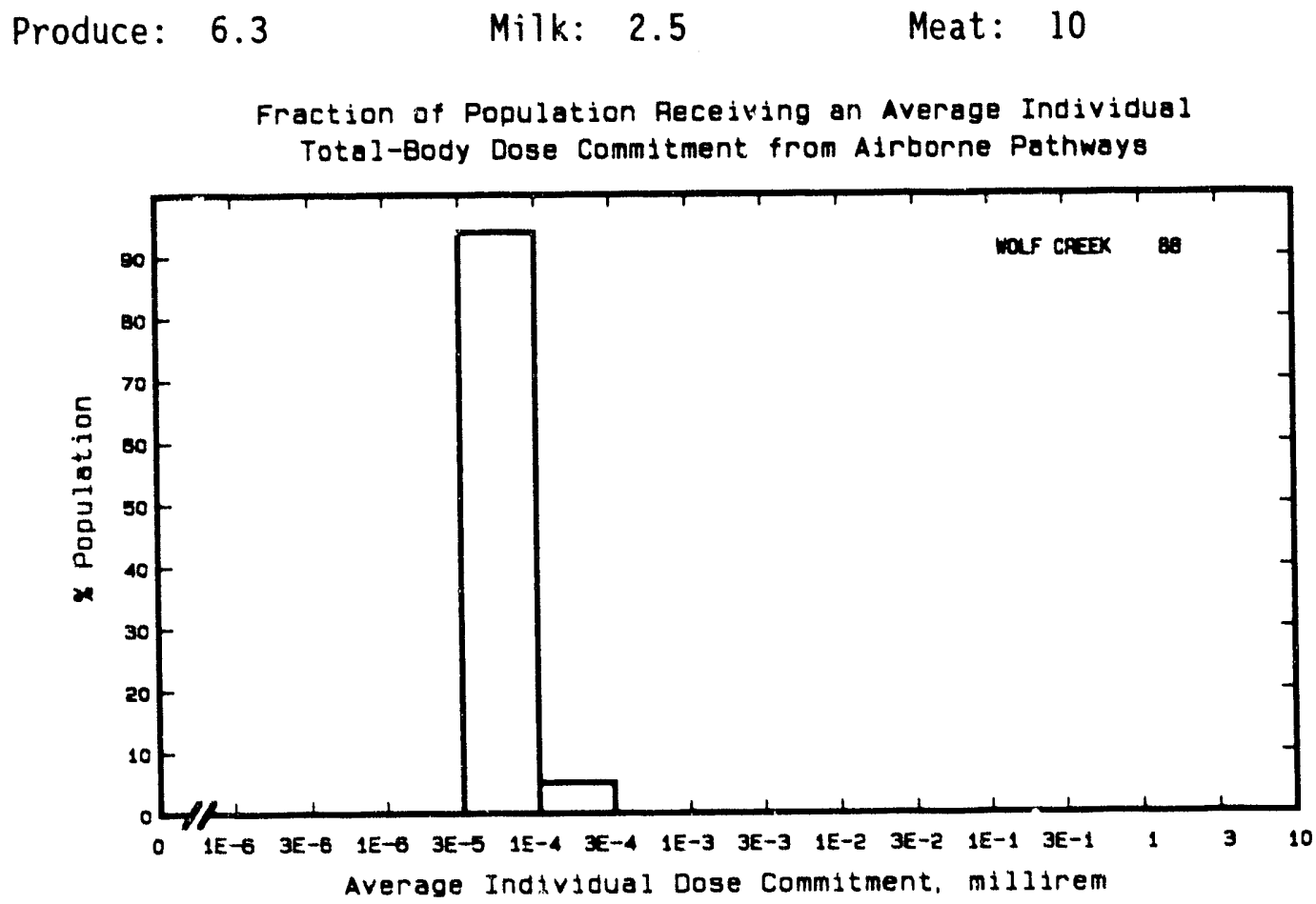


Site: YANKEE ROWE

ROWE, MASSACHUSETTS

Location: $\quad$ N $42.7281^{\circ}$ W $72.9289^{\circ}$

POPULATION DATA

Total Population Within 2-to-80-km Region: $1.6 \mathrm{E} 6$

Major Metropolitan Centers Within Region:

Center

Population

Location

Springfield-Chicopee-Holyoke SMSA

530,000

Albany

Pittsfield SMSA

100,000

92,000

Troy

57,000

Amherst

18,000

$74 \mathrm{~km} \quad \mathrm{SSE}$

$68 \mathrm{~km} \mathrm{~W}$

$41 \mathrm{~km} \mathrm{SW}$

$62 \mathrm{~km} \mathrm{~W}$

$51 \mathrm{~km} \quad \mathrm{SE}$

\section{SITE-SPECIFIC DATA - AIRBORNE PATHWAYS}

Average Annual State Production

of Crops and Animal Products

In $80-\mathrm{km}$ Radius Circle

Regional Productivity Factor:

Animal Grazing Factor:
Veg: $2.0 \mathrm{E} 7$ kilogram

Milk: 2.6E8 liter

Meat: $1.6 E 7$ kilogram

1

0.5

Meteorology Period of Record: 1 OCT 71 - 30 SEP 72 Recovery: $94 \%$

SITE-SPECIFIC DATA - WATERBORNE PATHWAYS via DEERFIELD RIVER

Average River Flow

at Site: $570 \mathrm{ft}^{3} / \mathrm{s}$

Drinking Water: None

Fish:

Edible Harvest: (a)

Dilution Factor: 0.025 (b)

(a) No catch data available, so gerieric consumiption rates used (Table A-1).

(h) Ten perrent nf mopulation obtain $25 \%$ of their fish from river. 


\section{POPULATION DOSE-COMMITMENT ESTIMATES AND AVERAGE INDIVIDUAL DOSE-COMMITMENT HISTOGRAM FOR \\ YANKEE ROWE}

Dose Commitments (person-rem) from Waterborne Pathways

Total Body GI-LII Thyroid Bone Liver

$\begin{array}{llllll}\text { Infant } & 0.0 \mathrm{E}+00 & 0.0 \mathrm{E}+00 & 0.0 \mathrm{E}+00 & 0.0 \mathrm{E}+00 & 0.0 \mathrm{E}+00 \\ \text { Child } & 3.7 \mathrm{E}-02 & 2.3 \mathrm{E}-02 & 2.2 \mathrm{E}-02 & 1.7 \mathrm{E}-01 & 9.9 \mathrm{E}-02 \\ \text { Teen } & 4.1 \mathrm{E}-02 & 1.7 \mathrm{E}-02 & 1.3 \mathrm{E}-02 & 1.0 \mathrm{E}-01 & 7.9 \mathrm{E}-02 \\ \text { Adult } & 3.8 \mathrm{E}-01 & 1.1 \mathrm{E}-01 & 7.7 \mathrm{E}-02 & 5.9 \mathrm{E}-01 & 4.7 \mathrm{E}-01 \\ \text { TOTAL } & 4.5 \mathrm{E}-01 & 1.5 \mathrm{E}-01 & 1.1 \mathrm{E}-01 & 8.6 \mathrm{E}-01 & 6.5 \mathrm{E}-01\end{array}$

Dose Commitments (person-rem) from Airborne Pathways

\begin{tabular}{lcccccc} 
& Total Body & GI-LII & Thyroid & Bone & Liver & Lung \\
\cline { 2 - 4 } & $1.8 \mathrm{E}-03$ & $1.8 \mathrm{E}-03$ & $1.9 \mathrm{E}-03$ & $7.7 \mathrm{E}-03$ & $1.8 \mathrm{E}-03$ & $1.8 \mathrm{E}-03$ \\
Infant & $1.3 \mathrm{E}-02$ & $1.3 \mathrm{E}-02$ & $1.3 \mathrm{E}-02$ & $5.4 \mathrm{E}-02$ & $1.3 \mathrm{E}-02$ & $1.3 \mathrm{E}-02$ \\
Child & $4.8 \mathrm{E}-03$ & $4.8 \mathrm{E}-03$ & $4.9 \mathrm{E}-03$ & $1.7 \mathrm{E}-02$ & $4.8 \mathrm{E}-03$ & $5.0 \mathrm{E}-03$ \\
Teen & $1.9 \mathrm{E}-02$ & $1.9 \mathrm{E}-02$ & $2.9 \mathrm{E}-02$ & $5.3 \mathrm{E}-02$ & $1.9 \mathrm{E}-02$ & $1.9 \mathrm{E}-02$ \\
Adult & $3.8 \mathrm{E}-02$ & $3.8 \mathrm{E}-02$ & $3.9 \mathrm{E}-02$ & $1.3 \mathrm{E}-01$ & $3.8 \mathrm{E}-02$ & $3.9 \mathrm{E}-02$
\end{tabular}

Production/Consumption factors:

Produce: 0.063 Milk: 1.2 Meat: 0.12

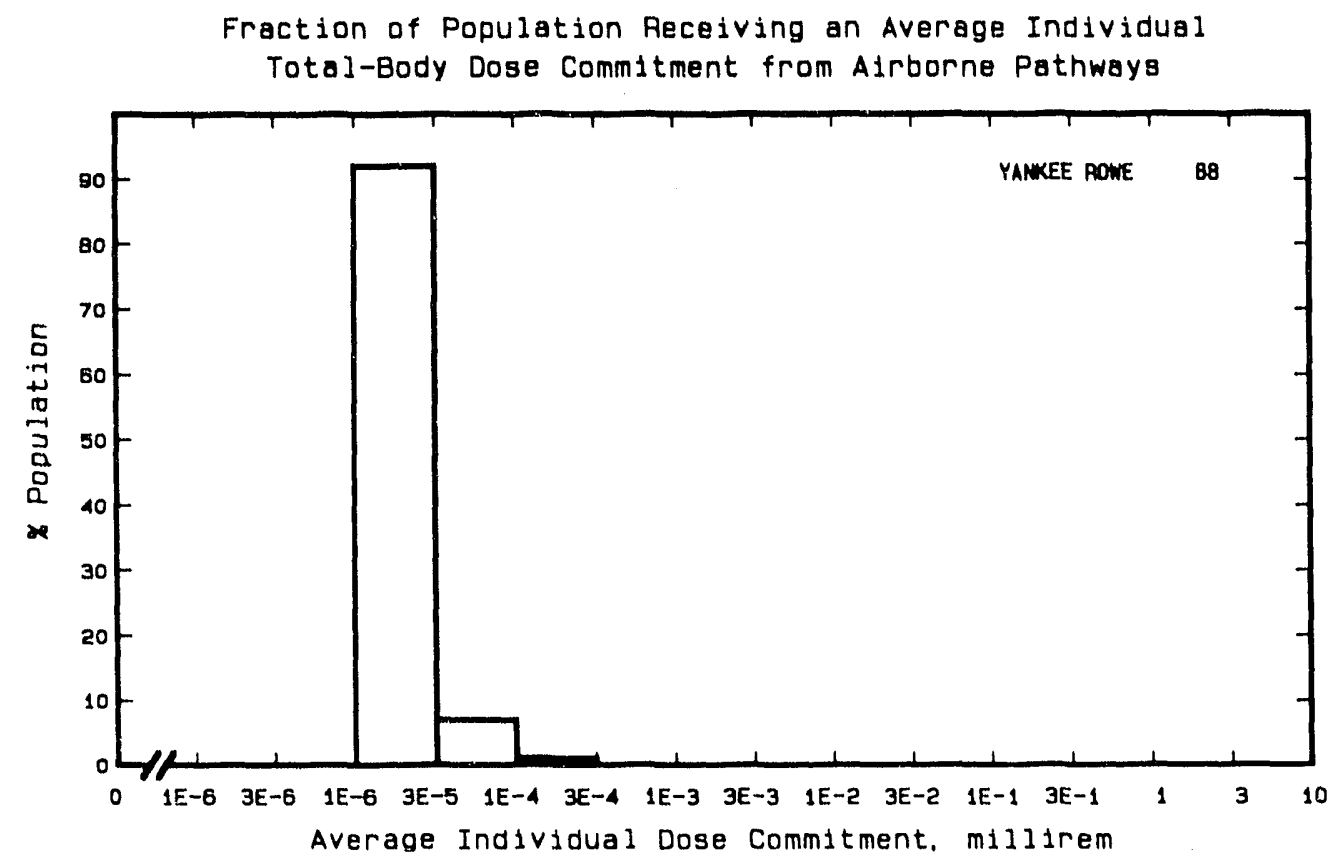


Site: ZION

ZION, ILLINOIS

Lucation: $\quad$ N $42.4456^{\circ}$ W $87.8022^{\circ}$

POPULATION DATA

Total Population Within 2-to-80-km Region: 7.3E6

Major Metropolitan Centers Within Region:

\begin{tabular}{|c|c|c|}
\hline Center & Population & Locat \\
\hline Chicago SMSA (2/3) & $4,800,000$ & $66 \mathrm{~km}$ \\
\hline $\begin{array}{l}\text { Milwaukee SMSA } \\
\text { Racine SMSA }\end{array}$ & $1,400,000$ & $65 \mathrm{~km}$ \\
\hline $\begin{array}{l}\text { Racine SMSA } \\
\text { Kenosha SMSA }\end{array}$ & 170,000 & $30 \mathrm{~km}$ \\
\hline Kenosha SMSA & 120,000 & $14 \mathrm{~km}$ \\
\hline Waukesha & 51,000 & $71 \mathrm{~km}$ \\
\hline
\end{tabular}

SITE-SPECIFIC DATA - AIRBORNE PATHWAYS

Average Annual State Production

Veg: $1.1 \mathrm{E8}$ kilogram

of Crops and Animal Products

Milk: 1.8E8 liter

In $80-\mathrm{km}$ Radius Circle

Meat: $1.9 \mathrm{E} 8$ kilogram

Regional Productivity Factor:

0.5

Animal Grazing Factor:

0.5

Meteorology Period of Record: 1 JAN 74 - 31 DEC 75 Recovery: $88 \%$

SITE-SPECIFIC DATA - WATERBORNE PATHWAYS via LAKE MICHIGAN

Average Dilution Flow

from Plant: $2,700 \mathrm{ft}^{3} / \mathrm{s}$

Drinking Water: $\quad$ Exposed Population: 6,900,000

Dilution Factor: $0.037(a)$

Fish:

Edible Harvest: $5.0 \mathrm{E} 6 \mathrm{~kg} / \mathrm{yr}$
Dilution Factor: $0.01 \mathrm{(b)}$

(a) Drinking water dilution factor est imated by averaging dilution factors derived from FES (1972) suitably weighted for population.

(b) Dilution factor derived from FES. 
POPULATION DOSE-COMMITMENT ESTIMATES AND

AVERAGE INDIVIDUAL DOSE-COMMITMENT HISTOGRAM FOR

ZION 1 AND 2

Dose Commitments (person-rem) from Waterborne Pathways

Total Body GI-LII Ihyroid Bone Liver

$\begin{array}{llllll}\text { Infant } & 9.2 \mathrm{E}-02 & 9.2 \mathrm{E}-02 & 1.6 \mathrm{E}-01 & 4.4 \mathrm{E}-02 & 1.4 \mathrm{E}-01 \\ \text { Child } & 1.1 \mathrm{E}+00 & 1.2 \mathrm{E}+00 & 1.4 \mathrm{E}+00 & 7.2 \mathrm{E}-01 & 1.8 \mathrm{E}+00 \\ \text { Teen } & 5.5 \mathrm{E}-01 & 6.3 \mathrm{E}-01 & 5.1 \mathrm{E}-01 & 2.7 \mathrm{E}-01 & 8.0 \mathrm{E}-01 \\ \text { Adult } & 5.1 \mathrm{E}+00 & 5.6 \mathrm{E}+00 & 4.1 \mathrm{E}+00 & 1.6 \mathrm{E}+00 & 5.7 \mathrm{E}+00 \\ \text { TOTAL } & 6.8 \mathrm{E}+00 & 7.5 \mathrm{E}+00 & 6.2 \mathrm{E}+00 & 2.7 \mathrm{E}+00 & 8.4 \mathrm{E}+00\end{array}$

Dose Commitments (person-rem) from Airborne Pathways

Total Body GI-LII Thyroid Bone Liver Lung

$\begin{array}{lllllll}\text { Infant } & 4.3 \mathrm{E}-03 & 4.3 \mathrm{E}-03 & 4.8 \mathrm{E}-03 & 1.7 \mathrm{E}-03 & 4.3 \mathrm{E}-03 & 4.4 \mathrm{E}-03 \\ \text { Child } & 6.9 \mathrm{E}-02 & 6.9 \mathrm{E}-02 & 7.4 \mathrm{E}-02 & 1.8 \mathrm{E}-02 & 6.9 \mathrm{E}-02 & 7.1 \mathrm{E}-02 \\ \text { Teen } & 5.2 \mathrm{E}-02 & 5.2 \mathrm{E}-02 & 5.4 \mathrm{E}-02 & 1.3 \mathrm{E}-02 & 5.2 \mathrm{E}-02 & 5.5 \mathrm{E}-02 \\ \text { Adult } & 3.1 \mathrm{E}-01 & 3.1 \mathrm{E}-01 & 3.2 \mathrm{E}-01 & 8.1 \mathrm{E}-02 & 3.1 \mathrm{E}-01 & 3.2 \mathrm{E}-01 \\ \text { TOTAL } & 4.4 \mathrm{E}-01 & 4.4 \mathrm{E}-01 & 4.5 \mathrm{E}-01 & 1.1 \mathrm{E}-01 & 4.4 \mathrm{E}-01 & 4.5 \mathrm{E}-01\end{array}$

Production/Consumption factors:

Produce: 0.040 Milk: 0.092 Meat: 0.16

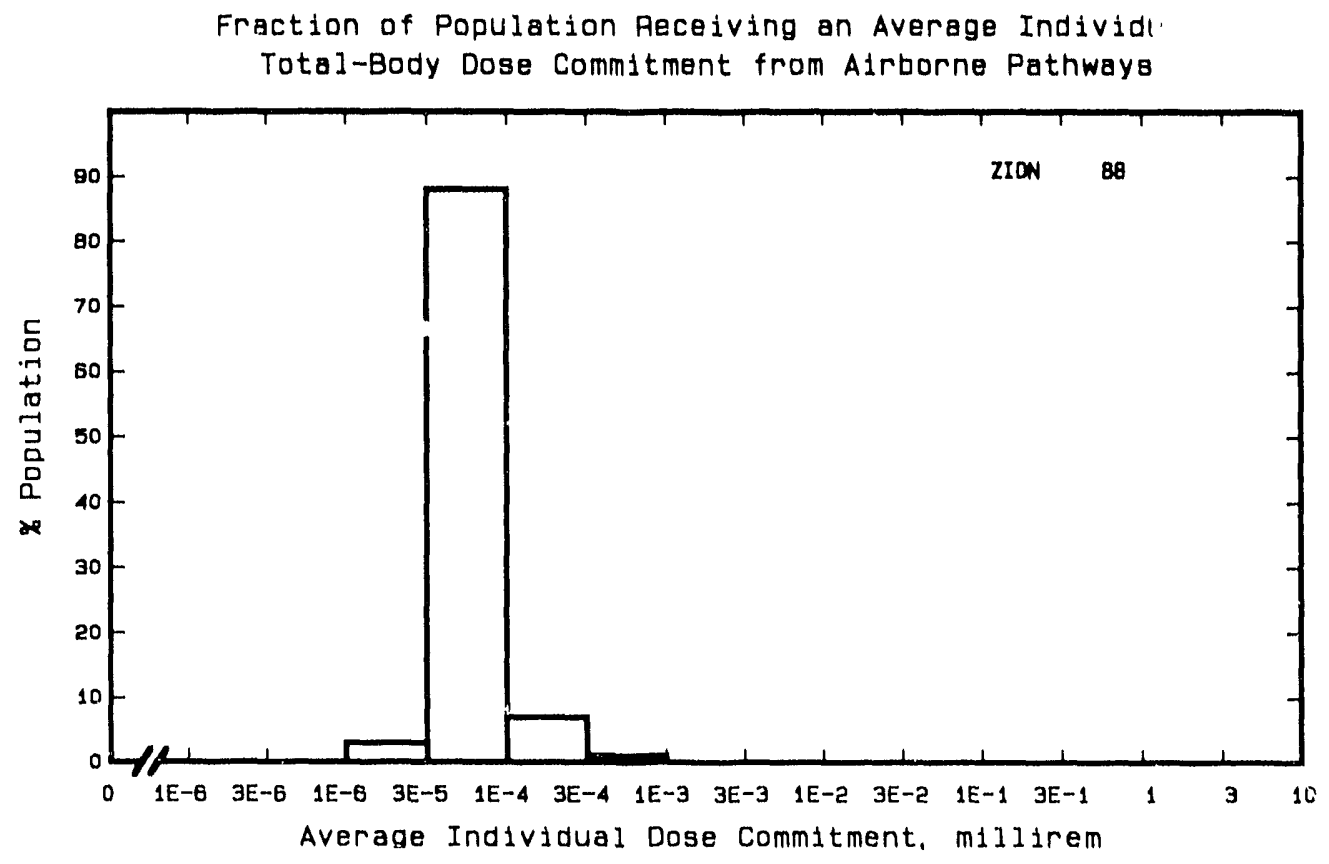




\section{REFERENCES}

Final Environmental Statement Concerning Proposed Rule-Making Action: Numerical Guides for Design Objectives and Limiting Conditions for Operation to Meet the Criterion "As Low As Practicable" for Radioactive Material in Light-Water-Cooled Nuclear Power Reactor Effluents. 1973. WASH-1258, Vol. 1, Directorate of Regulatory Standards, U.S. Atomic Energy Commission, Washington, D.C.

Tichler, J., K. Norden, and J. Congemi. 1991. Radioactive Materials Released from Nuclear Power Plants, Annual Report 1988. NUREG/CR-2907, BNL-NUREG-51581, Vo1. 9, U.S. Nuclear Regulatory Commission, Washington, D.C. 


\section{APPENDIX}

\section{MODELS AND GENERIC PARAMETERS}

The calculational models used were primarily those given in the Nuclear Regulatory Commission's Regulatory Guide 1.109, Rev. 1 (NRC 1977). Computer programs were written to use these models to generate population dose commitments for four age groups. The percentages of the population comprising the four age groups were $1.44 \%$, infant; $16.0 \%$, child; $11.7 \%$, teenager; and 70.9\%, adult (Population Estimates and Projections 1975). Where possible, the site- dependent parameters were taken from the environmental statements issued for each reactor (see Table 3). The generic parameters used for this study such as consumption rates, occupancy factors and holdup times are given in Tables $A-1$ and $A-2$ below. It should be noted that generic consumption rates for aquatic foods and inhalation rates are taken from Regulatory Guide 1.109 (NRC 1977). Bioaccumulation factors and terrestrial food transfer factors were taken from Regulatory Guide 1.109 (NRC 1977). Dose commitment factors for the four age groups were taken from Hoenes and Soldat (1977).

TABLE A-1. Generic Consumption Rates and Occupancy Factors Used for the Study of Average Members of the Population (a)

\begin{tabular}{|c|c|c|c|c|}
\hline Pathway & Infant & Child & Teen-ager & Adult \\
\hline $\begin{array}{l}\text { Fruits, vegetables and grain } \\
(\mathrm{kg} / \mathrm{yr})\end{array}$ & 0 & 200 & 240 & 190 \\
\hline $\operatorname{Mi} 1 \mathrm{k}(\mathrm{L} / \mathrm{yr})$ & 170 & 170 & 200 & 110 \\
\hline Meat and poultry $(\mathrm{kg} / \mathrm{yr})$ & 0 & 37 & 59 & 95 \\
\hline Fish $(\mathrm{kg} / \mathrm{yr})^{(\mathrm{b})}$ & 0 & 2.2 & 5.2 & 6.9 \\
\hline Invertebrates $(\mathrm{kg} / \mathrm{yr})$ & 0 & 0.33 & 0.75 & 1.0 \\
\hline Drinking water $(\mathrm{L} / \mathrm{yr})$ & $170^{(c)}$ & 260 & 260 & 370 \\
\hline Inhalation $\left(\mathrm{m}^{3} / \mathrm{yr}\right)$ & $1400^{(d)}$ & 3700 & 8000 & 8000 \\
\hline $\begin{array}{l}\text { Air submersion and ground } \\
\text { irradiation occupancy } \\
\text { factor }\end{array}$ & 0.5 & 0.5 & 0.5 & 0.5 \\
\hline
\end{tabular}

(a) Regulatory Guide 1.109 (NRC 1977).

(b) Both fresh and salt water.

(c) Assumed to be equal to milk consumption.

(d) Same as for maximum individual. 
IABLE A-2. Holdup Times Between Harvest and Consumption of Foods (a)

Food

Fruits, grains and vegetables

$M i 1 k(b)$

Meat (b)

Aquatic foods (fish and invertebrates)

Drinking Water
Holdup Time (days)

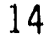

4

20

7

1

(a) Regulatory Guide 1.109 (NRC 1977)

(b) Value given is time after milking or slaughter. For the portion of the time animals were fed stored feed, an additional 90 days was added to the holdup time

\section{SOURCE TERMS}

The doses were estimated using the measured releases as reported by the site operators for 1988 (Tichler, et al. 1991). (a) These releases include a11 radionuclides specified by the NRC to be measured and reported by the operators of a 11 commercial nuclear power plants. Radionuclides given as a combination of parent-daughter isotopes such as $\mathrm{Y} / \mathrm{Sr}-90, \mathrm{Zr} / \mathrm{Nb}-95, \mathrm{Ba} / \mathrm{La}-140$, $\mathrm{I} / \mathrm{Xe}-133$ and $\mathrm{Pr} / \mathrm{Ce}-144$ were divided evenly between the parent and daughter.

The radionuclides used in this study, along with their decay constants, are given in Table $A-3$. Note that the " $+D$ " after some of the nuclides indicates that the decay energy of the daughter is included with the parent. Thus, whenever a parent nuclide release is specified, the result of the dose calculation is as though an additional equilibrium amount of the daughter nuclide is specified. The daughter nuclide itself is included separately if it can be released independently of the parent and/or if it has a relatively long half-life.

\section{METEOROLOGY}

When more than one set of meteorological (joint frequency) data was available for a site, the one which appeared to be the most reliable was used to generate atmospheric transport factors. Factors were calculated for 16 compass points and for ten radij from 2 to $80 \mathrm{~km}$ (see Table A-4) using the NRC computer program XOQDOQ (Sagendorf and Go11 1977).

(a) Very shortlived isotopes such as $\mathrm{Kr}-90,91,93,94, \mathrm{Xe}-139,140,141,143$ and Rb-88M; those not likely to be produced; and those that were daughters whose decay energies were accounted for in the dose factor for the parent were not included in the dose. 
TABLE A-3. Radionuclides Constdered in This Study

\begin{tabular}{|c|c|c|c|c|c|}
\hline No. & Nuclide & $\begin{array}{l}\text { Decay Constant } \\
-(1 / \mathrm{sec}) \\
\end{array}$ & No. & Nuclide & $\begin{array}{c}\text { Decay Constant } \\
-(1 / \mathrm{sec})\end{array}$ \\
\hline 1 & $H-3$ & $1.78 E-09$ & 43 & $\mathrm{Nb}-97$ & $1.57 E-04$ \\
\hline 2 & $\mathrm{Be}-10$ & $1.37 E-14$ & 44 & Mo-99+D & $2.92 E-06$ \\
\hline 3 & $C-14$ & $3.83 E-12$ & 45 & TC-99M & $3.19 E-05$ \\
\hline 4 & $N-13$ & $1.16 E-03$ & 46 & $R u-103+D$ & $2.02 E-07$ \\
\hline 5 & $F-18$ & $1.05 E-04$ & 47 & $R u-106+D$ & $2.17 E-08$ \\
\hline 6 & $\mathrm{Na}-22$ & $8.44 E-09$ & 48 & $\mathrm{Ag}-110 \mathrm{M}+\mathrm{D}$ & $3.19 E-08$ \\
\hline 7 & $\mathrm{Na}-24$ & $1.28 E-0.5$ & 49 & $C d-115 M$ & $1.80 E-07$ \\
\hline 8 & $A r-41$ & $1.05 E-04$ & 50 & $C d-115$ & $3.60 E-06$ \\
\hline 9 & Sc -46 & $9.58 E-08$ & 51 & $S n-125+D$ & $8.31 E-07$ \\
\hline 10 & $C r-51$ & $2.89 E-07$ & 52 & $\mathrm{Sb}-124$ & $1.33 E-07$ \\
\hline 11 & $M n-54$ & $2.57 E-08$ & 53 & $S b-125+D$ & $8.06 E-09$ \\
\hline 12 & $M n-56$ & $7.47 E-05$ & 54 & $\mathrm{Te}-132+\mathrm{D}$ & $2.47 E-06$ \\
\hline 13 & $\mathrm{Fe}-55$ & $8.14 E-09$ & 55 & $\mathrm{Te}-133 \mathrm{M}+\mathrm{D}$ & $2.09 E-04$ \\
\hline 14 & $\mathrm{Fe}-59$ & $1.80 E-07$ & 56 & $I-131+D$ & $9.97 E-07$ \\
\hline 15 & Co- 57 & $2.97 E-08$ & 57 & $I-132$ & $8.42 E-05$ \\
\hline 16 & Co- 58 & $1.12 \mathrm{E}-07$ & 58 & $I-133+D$ & $9.25 E-06$ \\
\hline 17 & Co -60 & $4.17 E-09$ & 59 & I - 134 & $2.20 E-04$ \\
\hline 18 & $\mathrm{Ni}-57$ & $5.35 E-06$ & 60 & $I-135+D$ & $2.92 E-05$ \\
\hline 19 & $\mathrm{Ni}-63$ & $2.20 E-10$ & 61 & $\mathrm{Xe}-131 \mathrm{M}$ & $6.69 E-07$ \\
\hline 20 & $\mathrm{Ni}-65$ & $7.64 E-05$ & 62 & $\mathrm{Xe}-133 \mathrm{M}$ & $3.61 E-06$ \\
\hline 21 & $\mathrm{Cu}-64$ & $1.52 E-05$ & 63 & $x e-133$ & $1.52 E-06$ \\
\hline 22 & $Z n-65$ & $3.31 E-08$ & 64 & $\mathrm{Xe}-135 \mathrm{M}$ & $7.56 E-04$ \\
\hline 23 & $Z n-69 M+D$ & 1.39E-05 & 65 & $X e-135$ & $2.10 E-05$ \\
\hline 24 & As -76 & $7.32 E-06$ & 66 & $x e-137$ & $3.01 \mathrm{E}-03$ \\
\hline 25 & $\mathrm{Br}-82$ & $5.44 E-06$ & 67 & $X e-138+D$ & $8.14 E-04$ \\
\hline 26 & $K r-83 M$ & $1.04 E-04$ & 68 & Cs -134 & $1.07 E-08$ \\
\hline 27 & $K r-85 M$ & $4.31 E-05$ & 69 & $C s-136$ & $6.17 \mathrm{E}-07$ \\
\hline 28 & $K r-85$ & $2.05 E-09$ & 70 & $C s-137+D$ & $7.31 \mathrm{E}-10$ \\
\hline 29 & $\mathrm{Kr}-87$ & $1.52 E-04$ & 71 & Cs -138 & $3.58 E-04$ \\
\hline 30 & $K r-88+D$ & $6.89 E-05$ & 72 & $C s-139+D$ & $1.24 E-03$ \\
\hline 31 & $k r-89$ & $3.64 E-03$ & 73 & $\mathrm{Ba}-139$ & $1.39 E-04$ \\
\hline 32 & $R b-88$ & $6.53 E-04$ & 74 & $\mathrm{Ba}-140+\mathrm{D}$ & $6.28 \mathrm{E}-07$ \\
\hline 33 & $\mathrm{Rb}-89+\mathrm{D}$ & $7.61 \mathrm{E}-04$ & 75 & La-140 & $4.78 E-06$ \\
\hline 34 & $S r-89+D$ & $1.59 \mathrm{E}-07$ & 76 & $L a-141$ & $4.97 E-05$ \\
\hline 35 & $S r-90+D$ & $7.58 \mathrm{E}-10$ & 77 & $\mathrm{Ce}-141$ & $2.47 \mathrm{E}-07$ \\
\hline 36 & $S r-91+D$ & $2.03 E-05$ & 78 & $\mathrm{Ce}-144+\mathrm{D}$ & $2.83 E-08$ \\
\hline 37 & $S r-92+D$ & $7.11 E-05$ & 79 & $E u-152$ & $1.69 \mathrm{E}-09$ \\
\hline 38 & $Y-90$ & $3.01 E-06$ & 80 & $E u-154$ & $2.55 E-09$ \\
\hline 39 & $Y-91 M+D$ & $2.32 E-04$ & 81 & W- 187 & $8.06 E-06$ \\
\hline 40 & $2 r-95+D$ & $1.22 E-07$ & 82 & $T h-232+D$ & $1.57 \mathrm{E}-18$ \\
\hline 41 & $2 r-97+D$ & $1.14 \mathrm{E}-05$ & 83 & $\mathrm{~Np}-239$ & $3.42 E-06$ \\
\hline 42 & $\mathrm{Nb}-95$ & $2.29 E-07$ & & & \\
\hline
\end{tabular}


TABLE A-4. Radius Intervals and Midpoints for

Atrborne Dose Calculations ( $\mathrm{km})$

$\begin{array}{cc}\text { Interval } & \text { Midpoin } \\ 2-3 & 2.5 \\ 3-4 & 3.5 \\ 4-6 & 5 \\ 6-9 & 7.5 \\ 9-14 & 11.5 \\ 14-20 & 17 \\ 20-30 & 25 \\ 30-40 & 35 \\ 40-60 & 50 \\ 60-80 & 70\end{array}$

The XOQDOQ program generates four sets of atmospheric transport factors:

- average annual atmospheric dilution factors, which are not corrected for cloud depletion or radioactive decay

- dilution factors that are only corrected for decay assuming a 2.26-day half-life

- dilution factors that are corrected for depletion and for decay assuming an 8-day half-life

- relative deposition per unit area.

These factors were used to estimate the dose from airborne releases using methods similar to the NRC GASPAR program (Eckerman et a1. 1980). The transport factors used this year were the same as those used for the previous estimates. The assumptions used in the calculation of these transport factors were as follows:

- 50-m source height with no correction for plume rise or butlding wake effects

- semi-infinite cloud model with sector-average, Gaussian-plume dispersion

- no correction for terrain height variation.

Since information about height and locations at each site for the releases given in Tichler, et al. (1991) was unavailable, a single generic height of $50 \mathrm{~m}$ was used at each site for the release point. Because the heights and locations of releases are uncertain, estimates of dose to persons 
living within $2 \mathrm{~km}$ of the stte could be in sertous error; only persons living between 2 to $80 \mathrm{~km}$ from the site were included in the dose estimates.

POPULATION

The population distribution withtn 2 to $80 \mathrm{~km}$ around each site was determined from information supplied by the NRC from an updated reduction of 1980 census data (Sinisgal11 1982). Also the NRC supplied updated estimates of the number of people residing in major metropolttan centers within the $80 \mathrm{~km}$ regton around each site (Brauner 1982) and D. P. Cleary. (a) Population vartations between 1980 and 1986 (population factors) were derived from census data (U. S. Bureau of the Census 1988).

\section{FOOD PRODUCTION VERSUS FOOD CONSUMPTION}

The total food production for the region within $80 \mathrm{~km}$ around each site was the product of the NRC state-wide productivity figure for each state and a site productivity factor. At some sites this total production may be more or less than the total consumption; f.e., population times average individual consumption (see Table A-1 for generic consumption rates). When production was more than consumption for a site, it was assumed that all persons in the 2-to-80-km region ate contaminated food; when production was less than consumption, tt was assumed that difution would occur because uncontaminated food would be shipped into the area from outside. Thus, the calculated doses for a particular food type were reduced in proportion to the ratto of pro-duction divided by consumption (production/consumption less than 1).

The dose to persons outside the $80-\mathrm{km} 1 \mathrm{imtt}$ from food shipped out of the region, in the case of production being greater than consumption, is not included in this report because we are concerned only with the dose within the $80 \mathrm{~km}$ radius. These production/consumption factors are given for reference as footnotes to the tables showing airborne dose commitment in the site Summaries section.

\section{DRINKING WATER}

The population between 2 and $80 \mathrm{~km}$ distance from each plant site exposed to drinking water contaminated with released radionuclides was generally obtained from the environmental statement (ES) for the plant. For all sites located on salt water, it was assumed that no dose was received from drinking water. The generic consumption rates used for drinking water are given in Table A-1.

(a) Letter from D. P. Cleary, U.S. Nuclear Regulatory Commission, to D. A. Baker, Pacific Northwest Laboratory, June 1987. 
The radionuclide concentration in the drinking water consumed by a population downstream from a stte was usually estifmated assuming $100 \%$ mixing of the plant effluent with the river. For Takes, an overall dilution factor was estimated from dilution factors given in the ES for each population center along the shore (with in $80 \mathrm{~km}$ ) that consumes the contaminated lake water. These individual factors were weighted by population and averaged to obtain an effective dilution factor for the total population exposed to contaminated drinking water.

\section{AQUATIC FOOD}

Wherever posstble, the fish-catch data from the plant ES were used to est tmate aquatic food consumption rates for the population living within the region. When these data were not found in the ES or were considered unrealistic, the genertc values of Table A-1 were used.

The average radionucitde concentration of the waters in which this food was harvested was estimated assuming an additional dilution over the effluent flow from the reactor. For rtvers, it was assumed that the fish were caught in waters in which the plant effluent was completely diluted. For lakes, an additional factor as given in the ES was used; when none was given in the ES, a generic value of 0.01 was used. For ocean and bay sites, genertc values of 0.001 and 0.002 were used for fish and invertebrates, respectively, if the ES ylelded no values for these parameters. Invertebrates were not assumed to be caught in suffictent quantity at freshwater sites (rtver and lake) to affect the population dose and therefore were not included in the dose calculation. Any exceptions to these general guidelines are explained in the footnotes to the individual site summartes. 


\section{APPENDIX REEERENCES}

Baker, D. A., J.K. Soldat and E. C. Watson. 1977. Population Dose Commitments Due to Radioactive Releases from Nuclear Power Plant sites in 1975. PNL-2439, Pactfic Northwest Laboratory, Richland, Washington.

Brauner, A. 1982. Population Estimates, Nuclear Power Plant Nearby PopUlation Concentrations. U.S. Nuclear Regulatory Commisston, Washingtion, D.C.

Eckerman, K. F., F. J. Conge1, A. K. Roecklein and W. J. Pasctak. 1980. Users Guide to GASPAR Code. NUREG-0597, U.S. Nuclear Regulatory Commission, Washington, D.C.

Hoenes, G. R., and J. K. Soldat. 1977. Age-Specific Radiation Dose Commitment Factors for a One-Year Chronic Intake. NUREG-0172, U.S. Nuclear Regulatory Commisston, Washington, D.C.

Population Estimates and Projections, Current Population Reports. 1975. Sertes P-25, No. 541. U.S. Department of Commerce, Soctal and Economic Statistics Admintstration, Bureau of the Census.

Sagendorf, J.F., J. T. Go11, and W. F. Sandusky. 1982. XOQDOQ: Computer Program for the Meteorological Evaluation of Routine Effluent Releases at Nuclear Power Stations. NUREG/CR-2.919, PNL-4380, U.S. Nuclear Regulatory Commission, Washington, D.C.

Sinisgal11, A. 1982. 1980 Residential Population Estimates, 0-80 Kilometers for Nuclear Power Plants. U. S. Nuclear Regulatory Commisston, Washington, D.C.

Tichler, J., K. Norden, and J. Congemi. 1991. Radioactive Materials Released from Nuclear Power Plants, Annual Report 1988. NUREG-CR-2907, BNL-NUREG-51581, Vo1. 9, U.S. Nuclear Regulatory Commission, Washington, D.C.

U.S. Bureau of the Census. 1990. Statistical Abstract of the United States: 1990, 110th edition. U.S. Bureau of the Census, Washington, D.C.

U.S. Nuclear Regulatory Commission. 1977. Calculation of Annual Doses to Man from Routine Releases of Reactor Effluents for the Purpose of Evaluating Compliance with 10 CFR Part 50, Appendix I, Revision 1. Regulatory Guide 1.109, Washington, D.C. 


\section{DISTRIBUTION}

No. of

Coptes

OFFSITE

U.S. Nuclear Regulatory Commisston

Diviston of Technical Information and Document Control

7920 Norfolk Avenue

Bethesda, MD 20014

Steve Baker

ICF Kaiser Engineers

601 Williams

Ktchland, WA 99352

Cathy Behling

263 0ld Limekiln Road

Chal sont, PA 18914

W. E. Bolch

University of Florida

Department of Environmental

Engineering Sciences

AP Black Hall

Gainesville, FL 32611

F. J. Borst

Fort St. Vrain Nuclear Generating Station

16805 Weld County Road 19 1/2

Plattevilie, CO 80651

K. R. Bräutigam

Kerns forschungszentrum Karlsruhe

7514 Eggenstein - Leopoldshafen

Karlsruhe

Federal Republic of Germany

H. D. Brenk

Zentralabteilung

Strahlenschultz der Kernforschungsanlage Jül ich GmbH

Postfach 1913 - 5170 Jütich 1

Federal Republic of Germany
No. of

Coptes

J. T. Brennen, M.D.

Radiation Management Corp.

3508 Market Street

Philladelphita, PA 19104

A. J. Col1t

Office of Air and Radiation

U.S. Environmental Protection Agency

Washington, DC 20460

R. M. DeNulman

Instituto Nacional De Investigaciones Nucleares

Benjamin Franklin 161

MEXCI0 11, D. F.

T. Dipierro

Butlding 475

Brookhaven National Laboratory

Upton, NY 11973

P. J. Dostie

State of Maine

Department of Human Services

Augusta, ME 04333

D. E. Dunning, Jr.

Evaluation Research Corporation 800 Oak Ridge Turnpike Suite 501

Oak Ridge, TN 37830

K. F. Eckerman

Oak Ridge National Laboratory

P.0. Box X

Oak Ridge, TN 37830

Ecological Sciences Information Center

Oak Ridge National Laboratory

Oak Ridge, TN 37830

Distr.1 
NUREG/CR-2850

PNL-4221

Vol. 10

No. of

Copies

T. H. Essig

Office of Nuclear Reactor

Regulation

U.S. Nuclear Regulatory Commission

Washington, DC 20555

R. J. Feinberg

Knolls Atomic Power Laboratory

General Electric Company

Box 1072

Schenectady, NY 12301

B. Feldman

Energy Systems Reserach Group

120 Milk Street

Boston, MA 02107

J. W. Ferman

Division of Wer Quality

Minnesota Pollution Control Agency

1935 West County Road B-2

Roseville, MN 55113

J. F. Greene

Mecklenburg County Department of Environmental Health

1200 Blythe Boulevard

Charlotte, NC 28203
No. of

Copies

F. 0. Hoffman

Oak Ridge National Laboratory P.0. Box $X$

Oak Ridge, in 37830

Mark Howard

Congressional Info. Service

4520 EW Highway

Suite 800

Bethesda, MD 20814-3389

L. Huebner

Hazleton Environmental Sciences

1500 Frontage Road

Northbrook, IL 60062

A. P. Hull

Brookhaven National Laboratory Upton, NY 11973

J. Iacovino

Nuclear Safety and Licensing

Westinghouse Electric Corporation

Box 355

Pittsburg, PA 15230

M. L. Joshi

Government of India

Department of Atomic Energy

Rajasthan Atomic Power Project Nuclear Training Centre

INDIA

B. Kahn

Environmental Resources Center Georgia Institute of Technology Atlanta, GA 30332

M. Laraia

C.N.E.N., Disp/Centr

00198 Roma

Viale Regina Margherita, 125

ITALY

L. Lewis

Duke Power Company

422 S. Church Street

Charlotte, NC 28242 
NUREG/CR-2850

PNL-4221

Vol. 10

No. of

Copies

S. Matts son

Lunds Universitet

Radi ofys iska Institutionen

Lasarettet, S-221 85 Lund

SWEDEN

\section{H. R. Meyer}

Oak Ridge National Laboratory

Oak Ridge, TN 37830

\section{J. Nagy}

Building 475

Brookhaven National Laboratory

Upton, NY 11973

S. Morris

Building 475

Brookhaven National Laboratory Upton, NY 11973

L. Namestek, Ing.

Prager Gasse 11/5

A-3002 ?urkersdorf

AUSTRI,

K. K. Narayanan

Bhabha Atomic Research Centre

Bombay - 400085

INDIA
W. Ogg
PSD CRBRP
P.0. Box U
Oak Ridge, TN 37830
G. V. 0ldfield
WPPSS
Mai1 Drop 1020
P. 0. Box 968
Richland, WA 99352
K. C. Pillai
Health Physics Division
Bhabha Atomic Research Centre
Bombay - 400085
INDIA

No. of

Copies

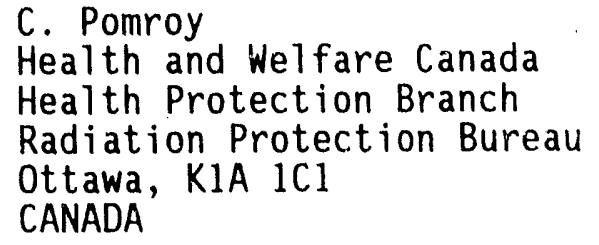

G. Poretti

$A B T$. F. MEDIZ. Strahlenphysik Inselspital

$\mathrm{CH}-3010$ BERN

SWITZERLAND

J. W. Poston

School of Nuclear Engineering Georgia institute of Technology Atlanta, GA 30332

R. R. Roselius

Consumers Power Company

Midland Plant Operations

P.0. Box 1593

Midland, MI 48640

P. Ross

Congressional Information

Service

4520 East West Highway

Washington, DC 20014

V. Sailor

Building 701

Brookhaven National Laboratory Upton, NY 11973

Leland Schneider

American Nuclear Insurers

The Exchange, Suite 245

270 Farmington Avenue

Farmington, CT 06032

J. Tadmor

Advisory Committee for

Nuclear Safety

Soreq Nuclear Research Centre

Yavne

ISRAEL 
NUREG/CR-2850

PNL-4221

Vol. 10

No. of

Copies

T. Thomas

National Institutes of Health Landow Bui iding, Room 3C 16

9000 Rockville Pike

Bethesda, MD 20014

J. Tichler

Building 51

Brookhaven National Laboratory Upton, NY 11973
A. N. Tshaeche
Mail 682
General Electric Company
175 Curtner Avenue
San Jose, CA 95125
F, Valerio
Universita Di Genova
Instituto Di Oncologia
16132 Genova
Viale Benedetto XV N.10
ITALY

P. Vasudev

Environment Canada

Place Vincent Massey

45th Floor

Ottawa, K1A 1 C8

CANADA

G. H. Whipple

3301 Rutland Loop

Tallahassee, FL 32312

E. I. White

9139 S. Bentham Avenue

Sandy, UT 84093

C. A. Willis

Office of Nuclear Reactor Regulation

U.S. Nuclear Regulatory Commission

Washington, DC 20555

F. E. Yost

Utility Data Institute, Inc. 2011 I Street, NW, Suite 700

Washington, DC 20006
No of

Copies

ONSITE

2 Westinghouse Hanford Company

H. R. Brager HO-36

P. R. Prevo N1-73

22 Pacific Northwest Laboratory

W. J. Bair K1-50

D. A. Baker (10) K3-54

T. L. Brown K1-35

J. R. Johnson K3-53

R. L. Moffitt K6-46

P. S. Stansbury K3-53

Health Physics Department Library

Publishing Coordination

Technical Report Files (5) 
(See instructions on the reverse)

\section{TITLE AND SUBTITLE}

Population Dose Commitments Due to Radioactive Releases

from Nuclear Power Plant Sites in 1988

5. AUTHOR(S)

D. A. Baker

Technical - Annual

8. PERFORMING ORGANIZATION - NAME AND ADDRESS III NRC, provicte Division, Office or Region, U.S. Nuclear Rugulatory Commission, and nialiling address: if controctor, provido nawe and mailing address.

Pacific Northwest Laboratory

Richland, WA 99352 9. SPONSORING ORGANIZATION - NAME AND ADDRESS III NAC, type "Seme as above": it contractor, provide NRC Division, Office or Region, U.S. Nuclear Regulatory Commission,

Division of Computer and Telecommunications Services

Office of Information Resources Management

U.S. Nuclear Regulatory Commission

Washington, DC 20555

10. SUPPLEMENTARY NUTES

11. ABSTRACT 1200 words or less)

Population radiation dose commitments have been estimated from reported radionuclide releases from commercial power reactors operating during 1988 . Fifty-year dose commitments from a one-year exposure were calculated from both liquid and atmospheric releases for four population groups (infant, child, teenager and adult) residing between 2 and $80 \mathrm{~km}$ from each of 71 sites. This report tabulates the results of these calculations, showing the dose commitments for both liquid and airborne pathways for each age group and organ. Also included for each of the sites is a histogram showing the fraction of the total population within 2 to $80 \mathrm{~km}$ around each site receiving various average dose commitments from the airborne pathways.

The total dose commitments (from both liquid and airborne pathways) for each site ranged from a high of 16 person-rem to a 10 w of 0.001 person-rem for the sites with plants operating throughout the year with an arithmetic mean of 1.1 person -rem. The total population dose for all sites was estimated at 75 person-rem for the 150 million people considered at risk.

12. KEY WOADS/DESCR!PTORS (L ist words or phrases that will assist nesearchers in locating the report.)

population dose commitments

radionuclide releases

liquid and atmospheric releases

\begin{tabular}{|c|}
\hline $\begin{array}{l}\text { 13. AVAILABILITY STATEMENT } \\
\text { Unlimited }\end{array}$ \\
\hline 14. SECURITY CLASSIFICATION \\
\hline $\begin{array}{l}\text { Thals Pagel } \\
\text { Uncl assified }\end{array}$ \\
\hline $\begin{array}{l}\text { UThis Report } \\
\text { Unclassified }\end{array}$ \\
\hline 15. NUMBER OF PAGES \\
\hline 16. PRICE \\
\hline
\end{tabular}



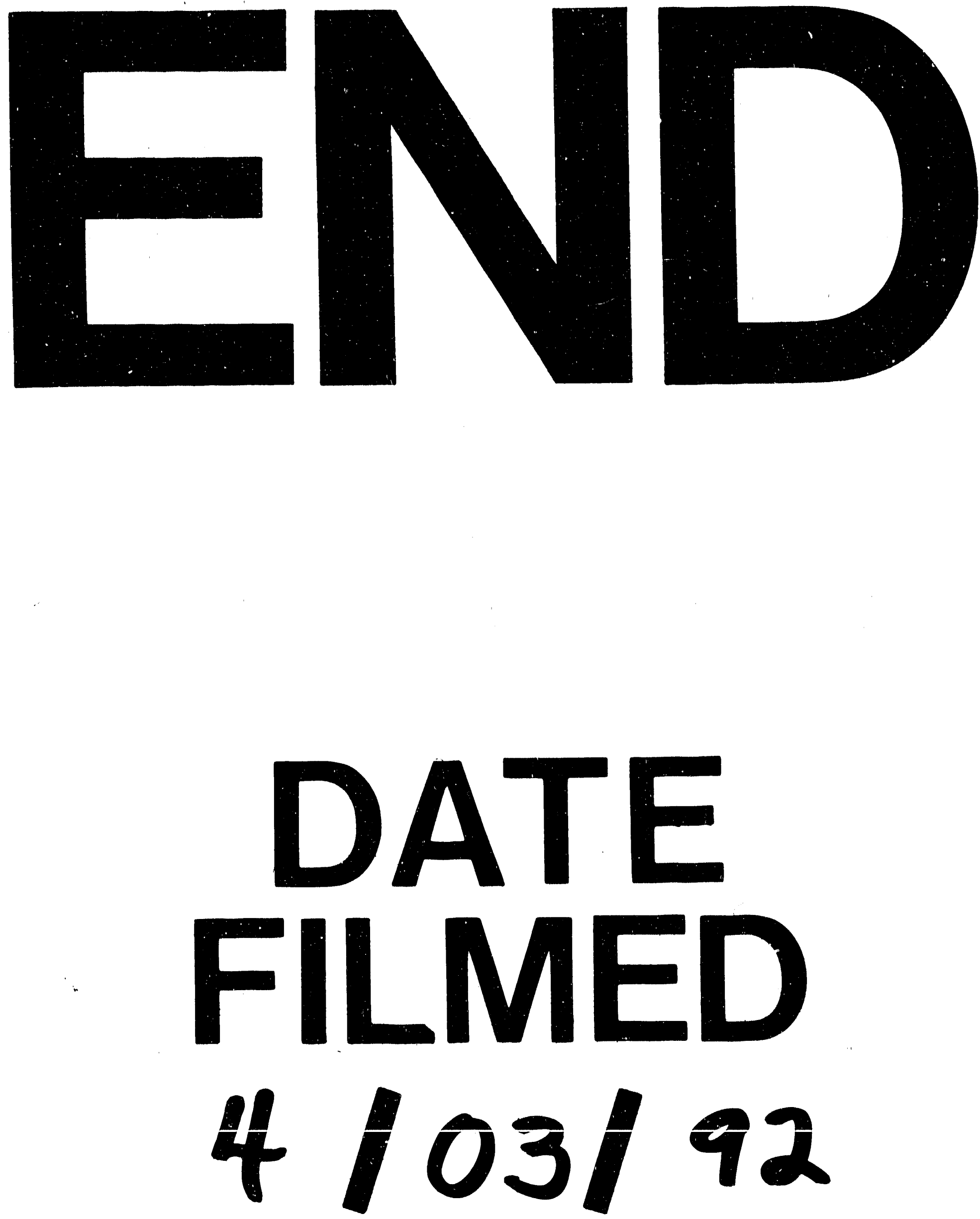

f 


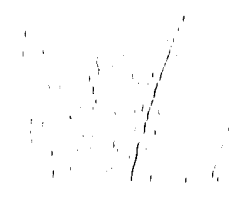

